$L B L-32233$

DE92 016925

\title{
Studies of Fundamental Properties of Rutherfordium (Element 104) Using Organic Complexing Agents
}

\author{
Kenneth R. Czerwinski \\ Ph.D. Thesis \\ Department of Chemistry \\ University of California \\ and \\ Nuclear Science Division \\ Lawrence Berkeley Laboratory \\ University of California \\ Berkeley, CA 94720
}

April 1992

This work was supported by the Dirctor, Office of Energy Research, Division of Nuclear Physics of the Office of High Energy and Nuclear Physics of the U.S. Department of Energy under Contract No. DE-AC03-76SF00098. 
Studies of Fundamental Properties of Rutherfordium (Element 104)

Using Organic Complexing Agents

by

\title{
Ken R. Czerwinski
}

\begin{abstract}
Chemical properties of rutherfordium (Rf) have been investigated with the organic ligands triisooctylamine (TIOA), tributylphosphate (TBP), and thenoyltrifluoroacetone (TTA). The TIOA studies showed that Rf behaves differently than Th and Eu and most similarly to $\mathrm{Zr}$, only $\mathrm{Zr}$ and Rf extract from 12 $\mathrm{M} \mathrm{HCl}$. This result is further evidence that $\mathrm{Rf}$ is a Group 4 element. Studies with TBP showed that Rf chemical behavior differed from the other Group 4 elements. The extraction by TBP at different chloride concentrations showed that $\mathrm{Rf}$ at times behaves more like $\mathrm{Pu}^{4+}$ than $\mathrm{Zr}$ or $\mathrm{Hf}^{4}$. At high chloride concentrations, $\mathrm{Rf}$ and $\mathrm{Pu}$ extraction decreased. Under the same conditions, $\mathrm{Zr}$, Hf and Th extraction increased. In addition, Rf extraction by TBP was affected by hydrogen ion concentration, while $\mathrm{Zr}$ and $\mathrm{Hf}$ extraction was not. TTA extractions were used to determine the $K_{e q}, K_{h y d}$, and the ionic radius of $R f$. The log $K_{e q}$ for Rf with TTA was calculated to be $3.18 \pm 0.90$. The first four log Khyd's for Rif are calculated to be $-2.6 \pm 0.7,-5.9 \pm 1.7,-10.2 \pm 2.9$, and $-14.5 \pm$ 4.1. These hydrolysis constants indicate that $R f$ will not hydrolyze at conditions under which $\mathrm{Zr}, \mathrm{Hf}$, and $\mathrm{Pu}$ will. Calculations of the ionic radius were made with the $\log K_{e q}$ for $R f$ derived with TTA. The ionic radius of $R f$ was calculated to be $91 \pm 4 \mathrm{pm}$ for the 6-coordinate species and $102 \pm 4 \mathrm{pm}$ for the 8-coordinate species. A search for the isotope ${ }^{263}$ Rf was conducted using TTA chemistry. In 300 experiments, seven SF events and no alpha events were observed in the Ri chemical fraction. The observed SF events were attributed to $263 \mathrm{R} f$ and had a half-life of $500 \pm 200$ seconds and a production cross section of $140 \pm 50 \mathrm{pb}$.
\end{abstract}




\section{Dedication}

This thesis is dedicated to two educators who inspired my curiosity in chemistry. Mr. George Herbert, my high school chemistry teacher, taught me the basics of chemistry and instilled in me a desire to learn more about this complex and exciting subject. Dr. Russell Sutton, my college chemistry advisor, encouraged my interest in research and helped further refine my knowledge of chemistry. I will always be deeply indebted to both these educators. 


\section{Acknowledgments}

I would first like to thank my advisors, Dr. Darleane C. Hoffman and Dr. Kenneth N. Raymond for their constant support. They provided me with the freedom and direction to develop this interdisciplinary work.

I would like to thank the members of the Raymond group for their help and friendship. I would like to especially thank Don Whisenhunt for his help with the program SPCONC.

My unlimited thanks go to the entire Hoffman group (Ken Gregorich, Diana Lee, Matti Nurmia, Andy Türier, Mary Neu, Bobby Kadkhodayan, Steve Kreek, Nancy Hannink, Todd Hamilton, Chris Kacher, Mike Lane, Eric Sylwester, and Reggie Gaylord). Without their help, none of the rutherfordium work would have been possible. Special thanks go to Dr. Ken Gregorich for all his help and scientific knowledge, Chris Kacher for his TBP work, and Mike Lane for his TTA work.

I am indebted to Dr. Heino Nitsche and Ray Gatti for their help and allowing me the use of their alpha scintillation system. This introduced me to a new spectroscopic method and greatly eased my alpha data analysis.

Finally, I would like to thank the staff of the LBL 88-Inch Cyclotron for the intense heavy ion beams they provided me.

This work was, at times, supported by the U.S. Department of Energy through the Director, Office of Energy Research, Division of Nuclear Physics of the Office of High Energy and Nuclear Physics under Contract DE-AC03-76SF00098. 


\section{Table of Contents}

Chapter 1 Introduction .......................................................................... 1

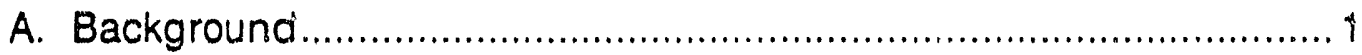

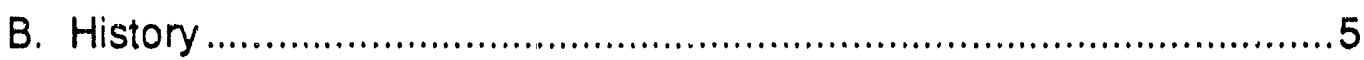

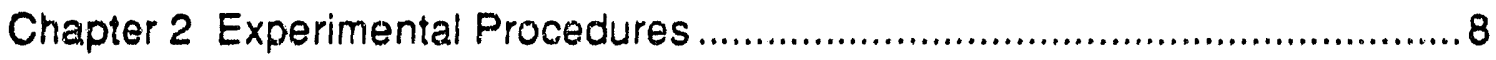

A. Cyclotron-Produced Isotopes...................................................... 8

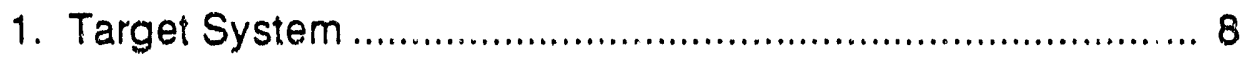

2. Target Preparation............................................................ 8

3. Gas-Jet Transport System.................................................. 9

4. Isotope Production............................................................ 15

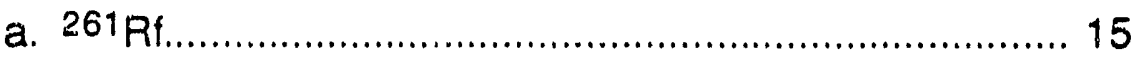

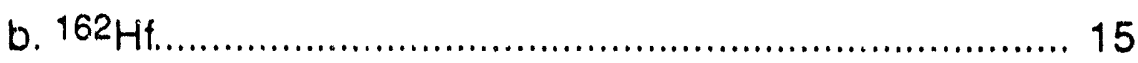

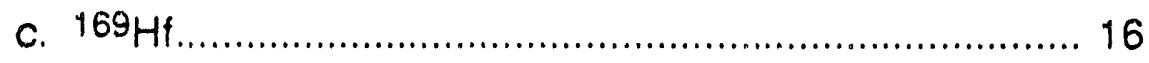

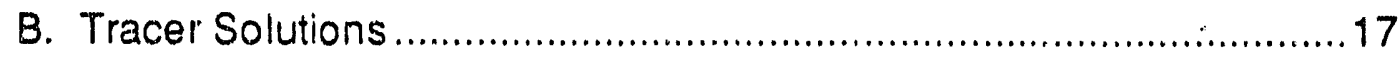

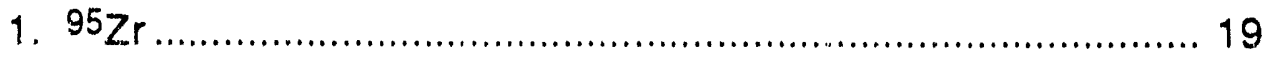

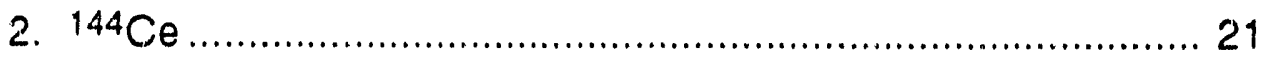

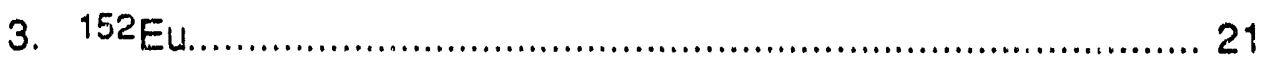

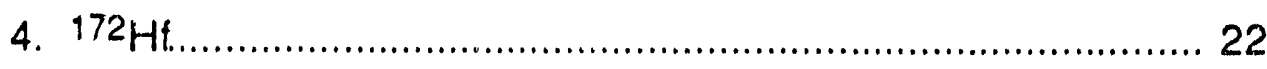

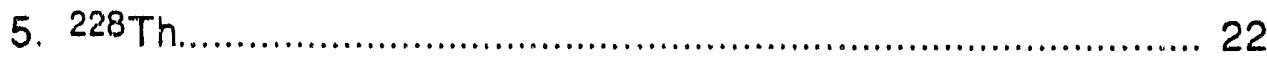

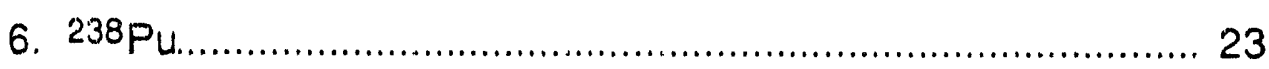

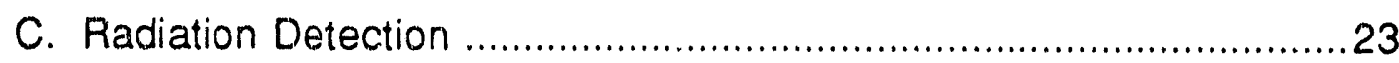

Chapter 3. Chemical Properties of Rutherfordium .........................................25

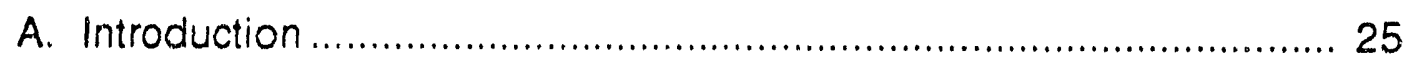

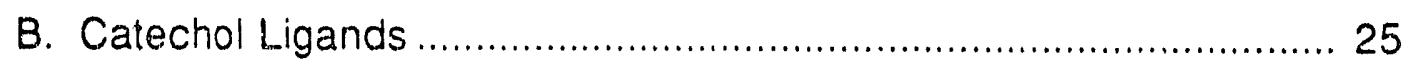

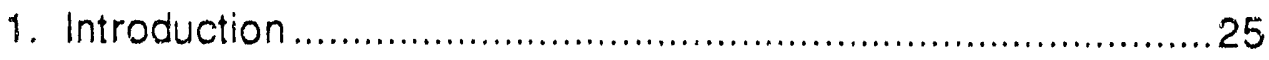

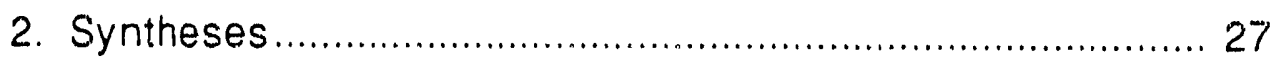

a. 2,3-Dimethoxybenzoyl chloride .................................. 28 
b. N,N',N",N"'-Tetrakis (2,3-dihydroxybenzoyl)-

1,5,10,14-tetraazatetradecane (3,4,3-LICAM)

c. N,N',N",N"'-Tetrakis(2,3-dihydroxy-5-sulfo-

benzoyl)-1,5,10,14-tetraazatetradecane (3,4,3-LICAMS) 30

d. N,N',N",N'"-Tetrakis(2,3-dihydroxy-5-nitro-

benzoyl)-1,5,10,14-tetraazatetradecane (3,4,3-LICAMN) 32

e. N,N',N",N"'-Tetrakis(2,3-dihydroxy-4-carboxy-

benzoyl)-1,5,10,14-tetraazatetradecane (3,4,3-LICAMC) 32

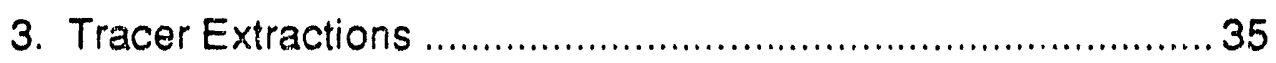

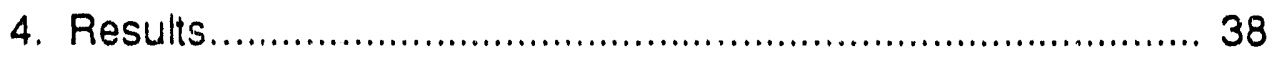

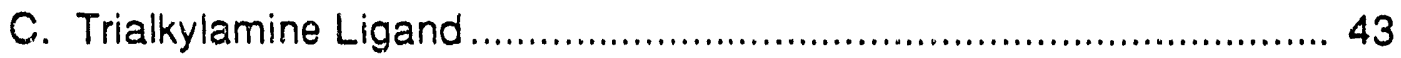

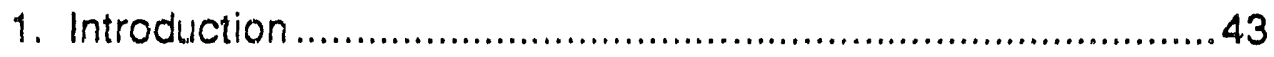

2. ${ }^{95} \mathrm{Zr},{ }^{95} \mathrm{Nb},{ }^{152} \mathrm{Eu}$, and ${ }^{228} \mathrm{Th}$ Extractions.......................... 45

3. ${ }^{95} \mathrm{Zr},{ }^{95} \mathrm{Nb},{ }^{152} \mathrm{Eu}$, and $228 \mathrm{Th}$ Results.............................. 46

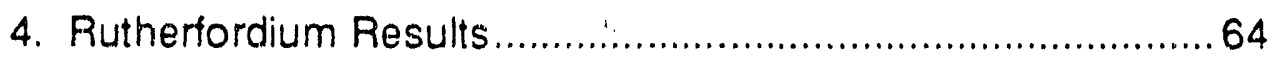

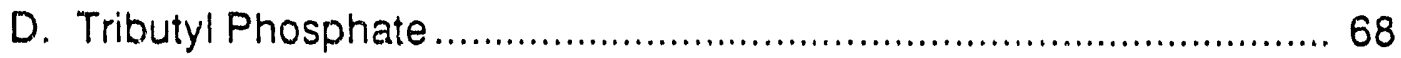

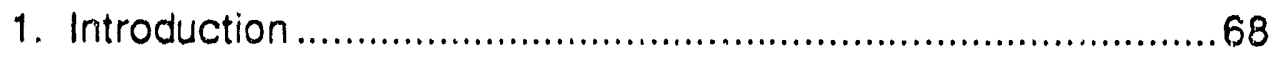

2. ${ }^{95} \mathrm{Zr}-95 \mathrm{Nb}$ Extractions ....................................................... 70

3. ${ }^{95} \mathrm{Zr}-95 \mathrm{Nb}$ Extraction Results and Conclusions.................... 72

4. Rutherfordium and Homolog Extraction Results ....................77

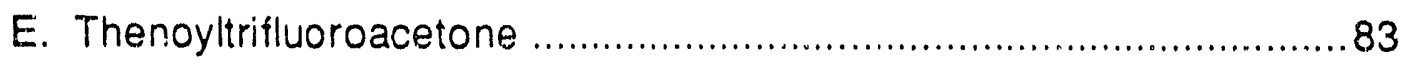

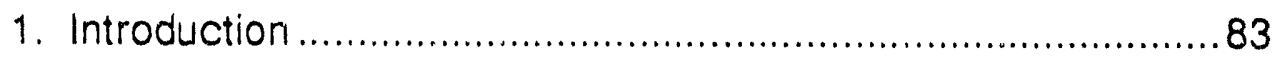

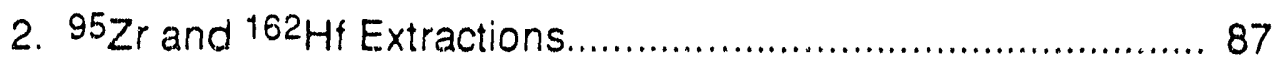

3. ${ }^{95} \mathrm{Zr}$ and ${ }^{162} \mathrm{Hf}$ Extraction Results..................................... 90

4. Rutherfordium and Homolog Extraction Results ....................91

5. Calculations ................................................................. 93

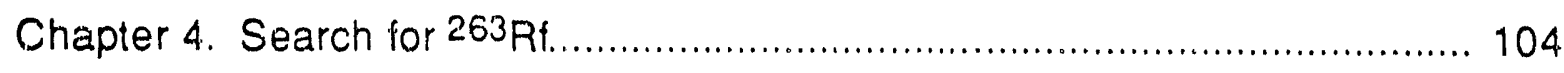




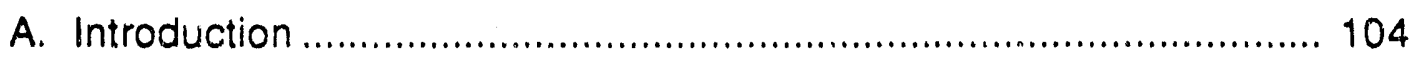

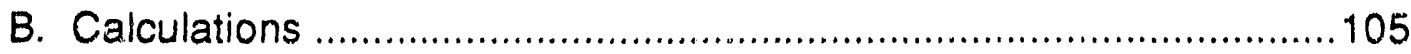

1. Cross Section .......................................................... 105

2. Alpha Decay .......................................................... 106

3. Electron Capture....................................................... 113

4. Spontaneous Fission .............................................. 114

C. Results and Discussion ........................................................ 116

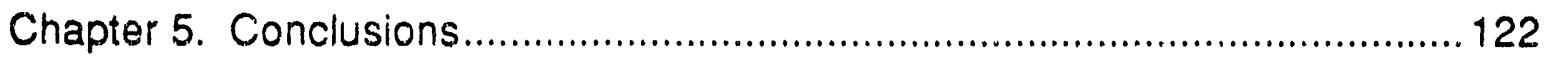

A. Chemical Studies ............................................................... 122

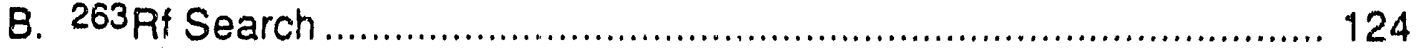

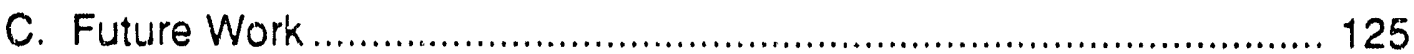

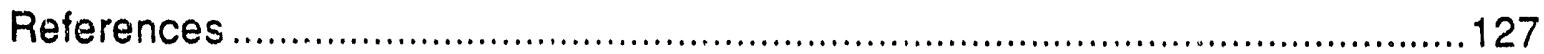




\section{List of Figures}

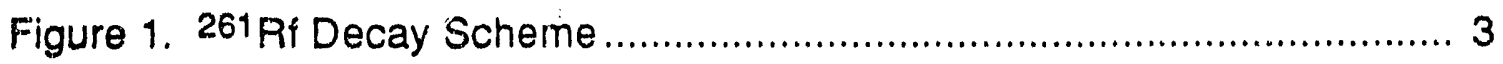

Figure 2. Target System...................................................................... 9

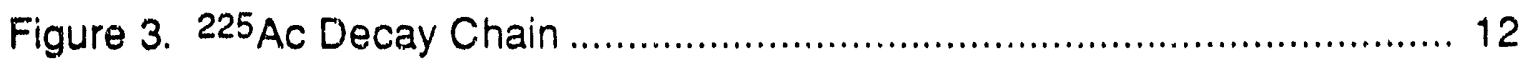

Figure 4. Effect of Oven Temperature on Gas Transport Yield........................ 14

Figure 5. Siderophore Functional Groups ................................................ 27

Figure 6. 2,3-Dimethoxybenzoyl Chloride Synthesis ....................................28

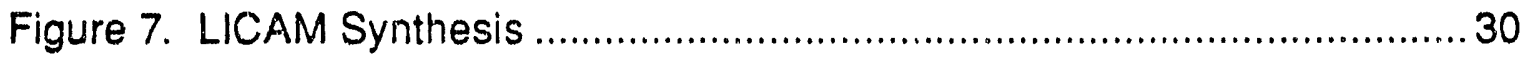

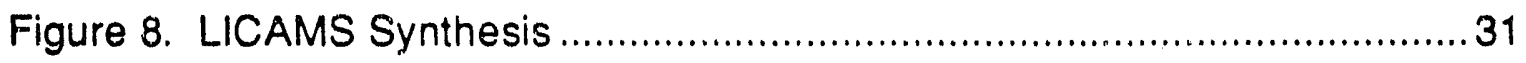

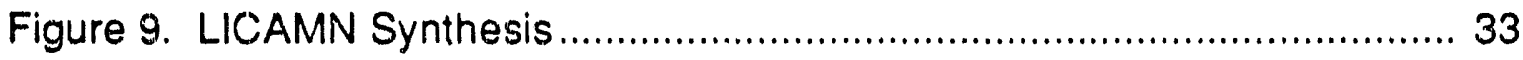

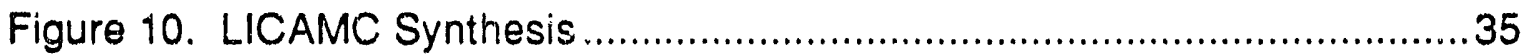

Figure 11. Effect of pH on Catechol-Metal Complexation .............................. 40

Figure 12. Effect of pH on LICAM-Metal Complexation ..................................40

Figure 13. Effect of pH on LICAMS-Metal Complexation ................................. 41

Figure 14. Effect of pH on LICAMN-Metal Complexation.............................. 41

Figure 15. Effect of pH on LICAMC-Metal Complexation............................... 42

Figure 16. Effect of pH on DTPA-Metal Complexation....................................4 42

Figure 17. Diluent Effect on $95 \mathrm{Zr}$ Extraction with TIOA ................................. 48

Figure 18. Diluent Effect on ${ }^{95} \mathrm{Nb}$ Extraction with TIOA ...................................48

Figure 19. Diluent Effect on 152Eu Extraction with TIOA.............................. 49

Figure 20. Effect of [Trihexylamine] on Tracer Extraction ............................. 49

Figure 21. Effect of [Triheptylamine] on Tracer Extraction............................. 50

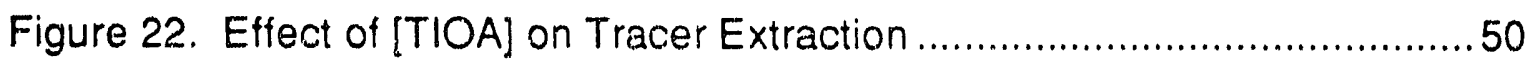

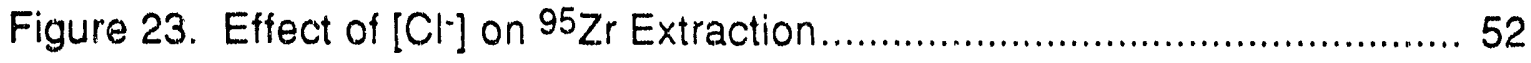

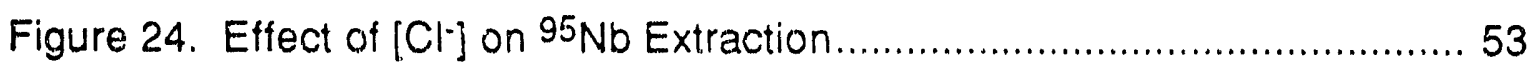

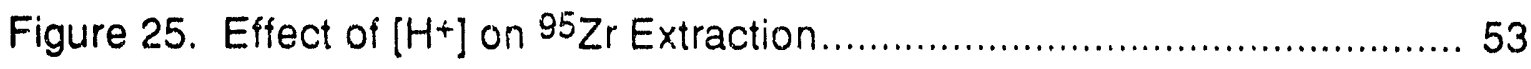

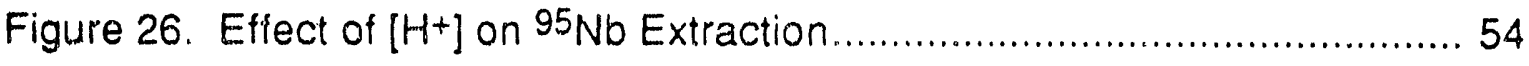




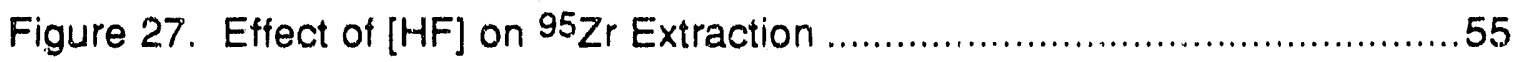

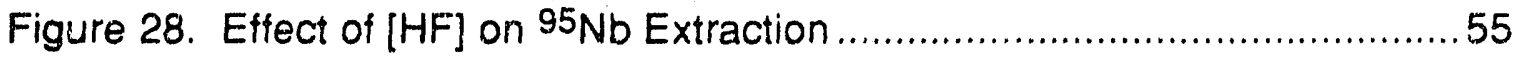

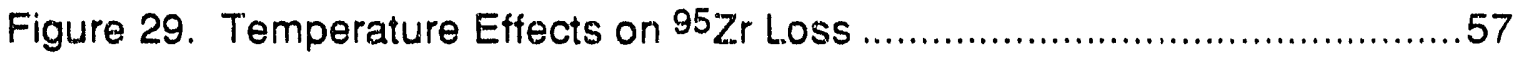

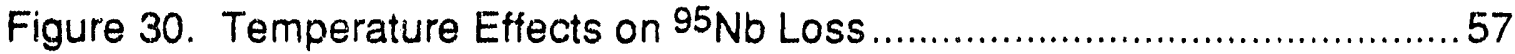

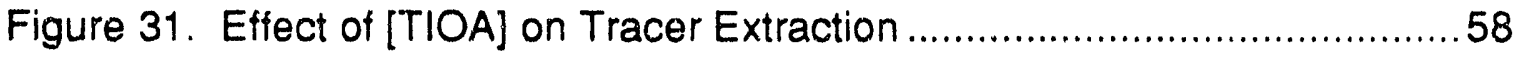

Figure 32. Effect of $[\mathrm{HCl}]$ on Tracer Extraction, No HF .................................59

Figure 33. Effect of $[\mathrm{HCl}]$ with $0.02 \mathrm{M} \mathrm{HF}$ on Tracer Extraction ......................60

Figure 34. Temperature Effects on $95 \mathrm{Zr}$ Loss with Varied [TIOA] ....................61

Figure 35. Effect of $\left[\mathrm{Cl}^{-}\right]$at $1 \mathrm{M} \mathrm{H}^{+} \mathrm{Cn}$ Tracer Extraction with TIOA ................62

Figure 36. Effect of [ $\left.\mathrm{Cl}^{-}\right]$at $7 \mathrm{M} \mathrm{H}^{+}$on Tracer Extraction with TIOA .................62

Figure 37. Effect of $\left[\mathrm{H}^{+}\right]$at $9 \mathrm{M} \mathrm{Cl}^{-}$on Tracer Extraction with TIOA .................63

Figure 38. Effect of $\left[\mathrm{H}^{+}\right]$at $12 \mathrm{M} \mathrm{Cl}$ on Tracer Extraction with TIOA ..............63

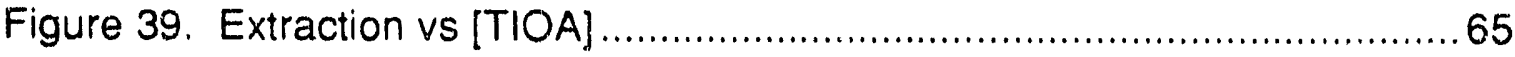

Figure 40. Effect of [TBP] on $95 \mathrm{Zr}-95 \mathrm{Nb}$ Extraction ..................................... 73

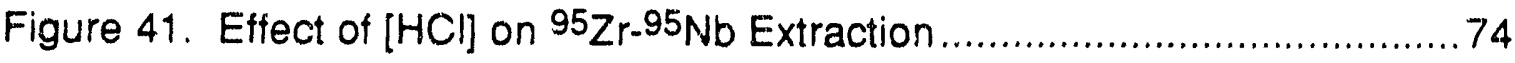

Figure 42. Effect of $[\mathrm{Cl}-]$ on $95 \mathrm{Zr}-95 \mathrm{Nb}$ Extraction.......................................... 74

Figure 43. Effect of $\left[\mathrm{H}^{+}\right]$on ${ }^{95} \mathrm{Zr}-95 \mathrm{Nb}$ Extraction....................................... 75

Figure 44. Effect of $\left[\mathrm{HNO}_{3}\right]$ on ${ }^{95} \mathrm{Zr}-95 \mathrm{Nb}$ Extraction ...................................

Figure 45. Effect of $[\mathrm{HCl}]$ on Extraction of $95 \mathrm{Zr},{ }^{169} \mathrm{Hf}, 228 \mathrm{Th}, 238 \mathrm{Pu}$, and $261 \mathrm{Rf}$

Figure 46. Effect of [ $\left.\mathrm{Cl}^{-}\right]$on Extraction of ${ }^{95} \mathrm{Zr},{ }^{169} \mathrm{Hf},{ }^{228} \mathrm{Th},{ }^{238} \mathrm{Pu}$, and $261 \mathrm{Rf}$

Figure 47. Effect of $\left[\mathrm{H}^{+}\right]$on Extraction of $95 \mathrm{Zr},{ }^{169} \mathrm{Hf}$, and ${ }^{261} \mathrm{Rf} \ldots \ldots \ldots \ldots \ldots \ldots . . . . . . . . . .28$

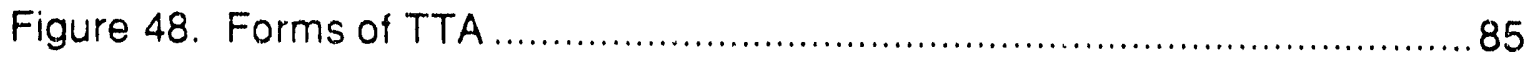

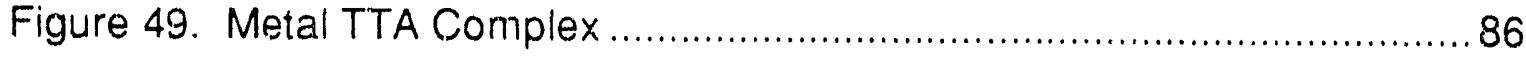

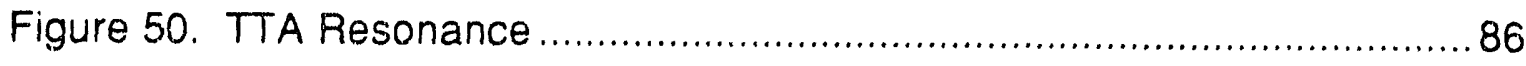

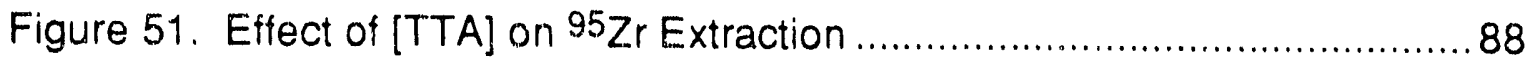




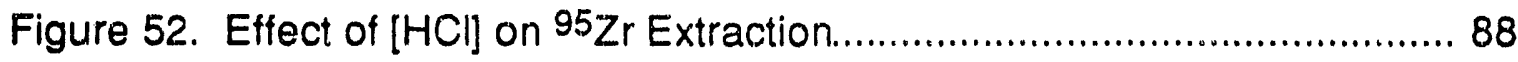

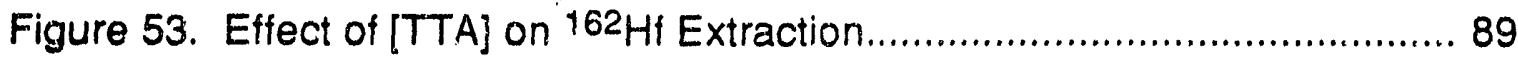

Figure 54. Effect of $[\mathrm{HCl}]$ on $162 \mathrm{Hf}$ Extraction............................................... 90

Figure 55. Effect of $[\mathrm{HCl}]$ on ${ }^{261} \mathrm{Rf}$ and Homologs Extraction ......................... 92

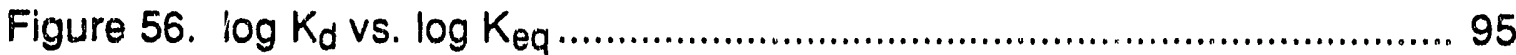

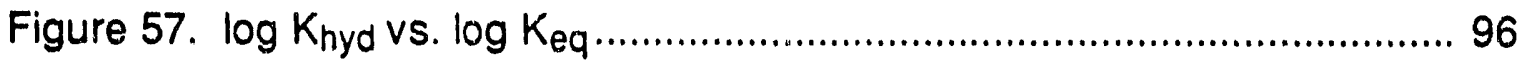

Figure 58. Zirconium Species Distribution................................................. 98

Figure 59. Hafnium Species Distribution.................................................... 99

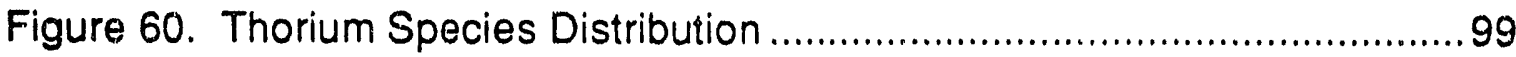

Figure 61. Plutonium Species Distribution ................................................100

Figure 62 Rutherfordium Species Distribution............................................. 100

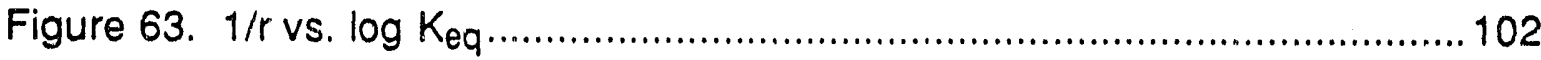

Figure 64 SPIT Cross Section Calculation for ${ }^{263}$ Rf Production...................... 106

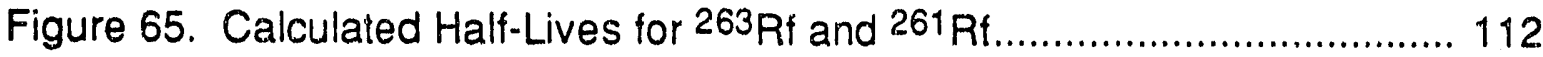

Figure 66. Woods-Saxon Potential Diagram For Protons ...............................116

Figure 67. Alpha Spectrum From 263 Rf Search ....................................... 118

Figure 68. Number of Alpha Events Expected ..............................................120

Figure 69. Probability of Alpha Decay Energy ........................................... 121 


\section{List of Tables}

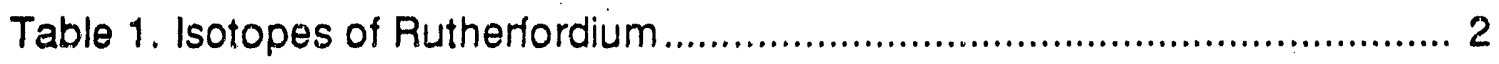

Table 2. Effect of Capillary Size on Gas-Jet Yield............................................ 12

Tabie 3. Effect of Gas Flow on Transport Yield ...........................................13

Table 4. ${ }^{162 H f}$ Decay Information.................................................................. 16

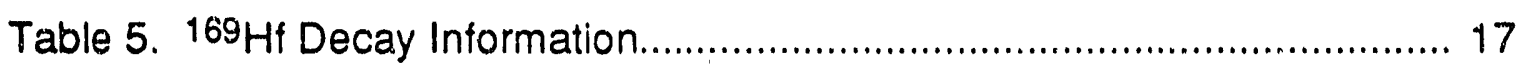

Table 6. ${ }^{95} \mathrm{Zr},{ }^{95} \mathrm{Nb}$, and ${ }^{152 E u}$ Decay Information...................................... 18

Table 7. 228Th and 238Pu Decay Information............................................... 18

Table 8. Neutral Atom Electronic Structure and Ionic Radius ........................ 19

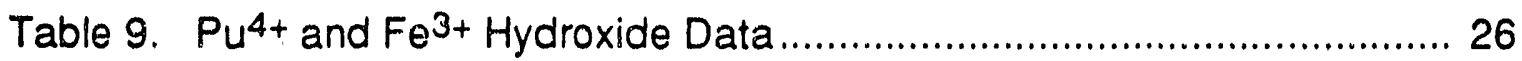

Table 10. Ce, Eu, and Th Charge Per lonic Radius .........................................28

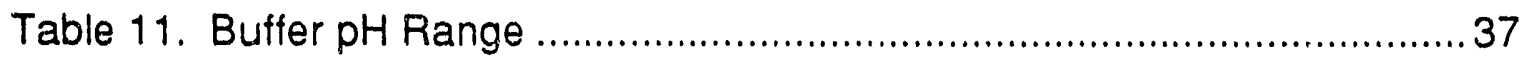

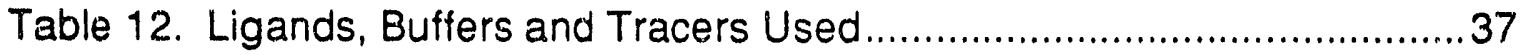

Table 13. Amine Extractant Data ............................................................... 45

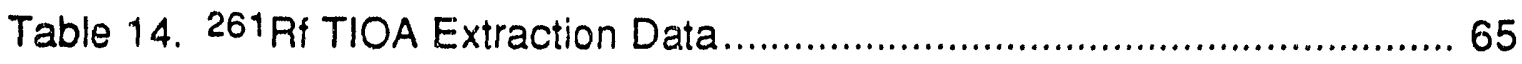

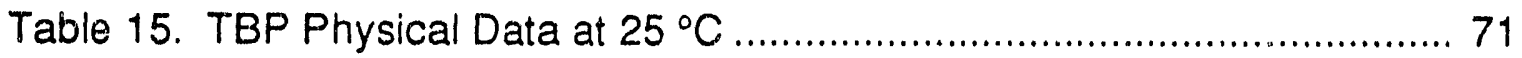

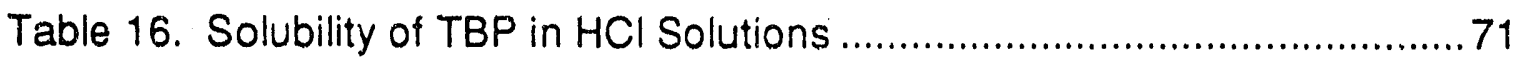

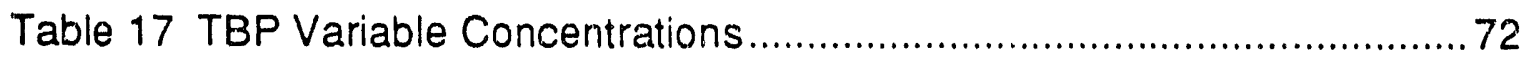

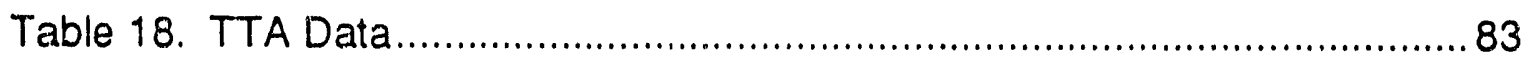

Table 19. Distribution Coefficients for $261 \mathrm{R} f$ and Homologs............................ 94

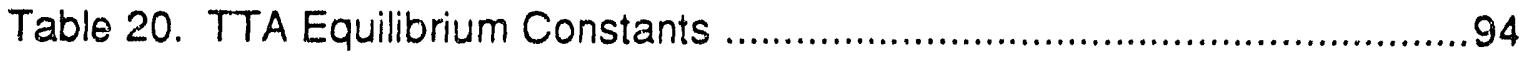

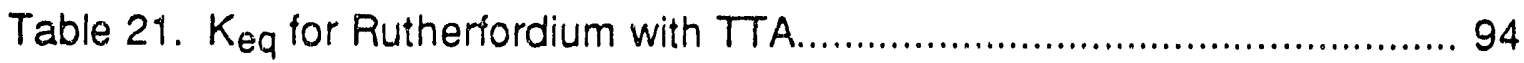

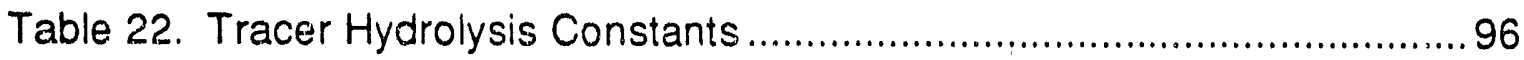

Table 23. Rutherfordium Hydrolysis Constants ........................................... 97

Table 24. Ionic Radius for Tetravalent Rutherfordium.................................... 102

Table 25. Increase in lonic Radius from 6-Coordinate to 8-Coordinate ........... 103

Table 26. $Q$ value Calculations ............................................................... 108 
Table 27. ${ }^{263} \mathrm{Rf}$ Alpha Decay Half-Life Estimate ....................................... 113

Table 28. Mass Excesses (MeV) of ${ }^{263} \mathrm{Rf}$ and $263 \mathrm{Lr}$ and EC Half-lives.......... 114 


\section{Chapter 1: Introduction}

\section{A. Background}

When examining elements, scientists typically look at two aspects: the electrons and the nucleus. In scientific investigations, these are usually examined separately. Chemists typically concern themselves with the interactions of the atomic electrons. Stability of the electron configuration determines chemical properties such as oxidation state, metal-ligand complexation, metal-ligand solution species and other chemical properties exhibited by the element or molecule. Research on the properties of the nucleus is usually the realm of physics and nuclear chemistry, which delve into nuclear structure, decay modes, masses, and other physical characteristics displayed by the nucleus.

However, in research on the chemistry of the heavy elements, the distinction between investigations of the nucleus and the electrons becomes blurred and interdependent. This is the realm of radiochemistry, the chemistry involved with the study of radioactive isotopes. Radiochemistry is vital to heavy element research. One can only investigate the chemical properties of a heavy element after identifying some nuclear properties, e.g., half-life, decay modes and energies of emitted radiations, have been determined. In addition, the nuclear properties of a heavy element nucleus can be further studied by utilizing chernistry as a tool to separate the desired element from the wide range of interfering product nuclei usually made in the nuclear reactions. Chemistry can aiso be used in the investigation and identification of new isotopes of the heavy elements.

Study of the transactinide elements $(Z>103)$ is a difficult matter due to the nature of the transactinide elements and the additional problems encountered when producing elements via heavy ion reactions which have very small cross 
sections. The isotopes of rutnerfordium decay by both alpha and spontaneous fission (SF). Spontaneous fission is the break up of a heavy nuclei into two lighter nuclei and neutrons. The known isotopes of the transactinide elements all have very short half-lives. For example, the longest-lived isotope of putherfordium (element 104) is 65-second $261 \mathrm{Rf}$ (Table 1). The short half-life of this isotope makes chemical experiments extremely difficult. To effectively study the chemistry of $261 \mathrm{Rf}$, chemical procedures must be carried out in a time comparable to the half-life. Additionally, the resulting samples must be suitable for radiation measurements. The isotope $261 \mathrm{Rf}$, produced via the $248 \mathrm{Cm}(180,5 \mathrm{n})$ reaction, has a cross section of only $5 \mathrm{nb}$ and is produced on an atom-at-a-time basis at an accelerator [GH170]. The low production rate further complicates rutherfordium chemistry by requiring repetitive and often tedious chemical procedures in order to obtain results of statistical importance.

\begin{tabular}{|c|c|c|}
\hline \multicolumn{3}{|c|}{ Table 1. Isotopes of Rutherfordium } \\
\hline Mass Number & Half-Life & $\begin{array}{l}\text { Decay Mode and } \\
\alpha \text {-Particle Energies (MeV) }\end{array}$ \\
\hline $253 ?$ & $\approx 1.8 \mathrm{~s}$ & $S F, \alpha ?$ \\
\hline $254 ?$ & $0.5 \mathrm{~ms}$ & SF \\
\hline 255 & $1.7 \mathrm{~s}$ & SF \\
\hline 256 & $7 \mathrm{~ms}$ & $S F, \alpha(8.81)$ \\
\hline 257 & $4.7 \mathrm{~s}$ & $\alpha(8,77,9.01,8.95,8.62)$ \\
\hline 258 & $12 \mathrm{~ms}$ & SF \\
\hline 259 & $3.4 \mathrm{~s}$ & $\alpha, \operatorname{SF}(8.77,8.86)$ \\
\hline 260 & $20 \mathrm{~ms}$ & SF \\
\hline 261 & $65 \mathrm{~s}$ & $\alpha(8.29)$ \\
\hline 262 & $52 \mathrm{~ms}$ & $S F$ \\
\hline
\end{tabular}

Reference [WAL89] 
The heavy ion reaction used to produce ${ }^{261} \mathrm{Rf}$ also yields a legion of actinides, many decaying by alpha emission. The ${ }^{261} \mathrm{Rf}$ must be separated from these alpha emitters since the rutherfordium is detected by its alpha decay. In addition, detection of the $261 \mathrm{R} f$ daughter, 257 No (Figure 1), can be used to verify the presence of the parent $261 \mathrm{Rf}$ only if any 257 No directly produced via the $248 \mathrm{Cm}(180, \alpha 5 \mathrm{n})$ reaction has first been removed. This limits the type of chemistry that can be performed to easily repeatable, rapid actinidetransactinide chemical separations from which thin samples for alpha spectroscopy can be quickly prepared.

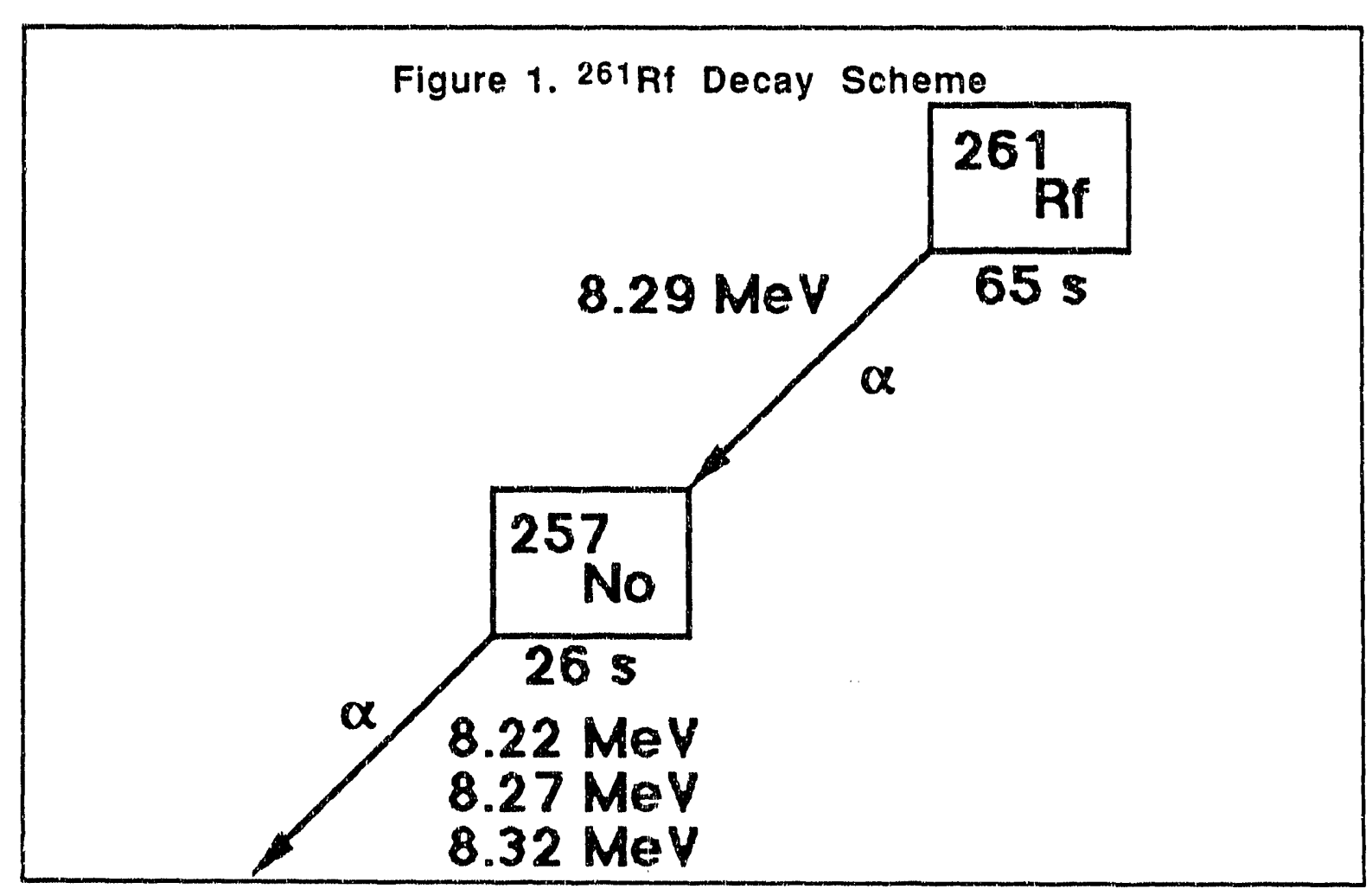

To comply with these mentioned limitations, liquid-liquid extractions with a variety of organic soluble ligands were performed on $261 \mathrm{Rf}$ and its lighter homologs. In addition, polydentate ligands were synthesized and tested with the rutherfordium homologs. Comparison of the chemical properties of 
rutherfordium to its lighter homologs is a powerful tool in understanding the chemical properties of rutherfordium. For example, by studying the solvent extraction properties of rutherfordium and comparing them to the extraction properties of its lighter homologs, zirconium and hafnium, information about, the extracted chemical species, complexing ibility, and ionic radius of rutherfordium can be obtained.

Comparison of the chemical properties exhibited by rutherfordium and the other transactinide elements with those of the lighter homolog elements can help in determining whether relativistic effects are important in defining the chemical properties of the transactinide elements. The relativistic effects arise from the high nuclear charge of the transactinide elements and may change the relative stability of the $7 s, 6 d$ and $7 p$ valence electrons. This, in turn, may alter the expected ionic radius. A recent review of relativistic effects [PYY88] states that the $p_{2 / 3}, d$ and $f$ electrons will be shielded from the high nuclear charge by the contraction of the $s$ and $p_{1 / 2}$ orbitals. The influence of relativistic effects on atomic orbitals should be contraction and energy stabilization of the $s$ and $p_{1 / 2}$ orbitals and increased radial extension and energy destabilization of the $d$ and $f$ orbitals. These effects are believed to be of the same order of magnitude and increase as $Z^{2}$.

Calculations of lawrencium atomic electronic structure based on multiconfiguration Dirac Fock (MCFD) calculations predicted a relativistic stabilization of the $7 p_{1 / 2}$ orbital [BRE84], [DES80], and a [Rn]5f $147 s^{2} 7 p_{1 / 2}$ electronic structure for lawrencium, the last of the actinides, rather than the $[R n] 5 f^{14} \varepsilon d 7 s^{2}$ configuration expected by extrapolation from Lu (the last of the lanthanides) which has the configuration $[X e] 5 f^{14} 6 \mathrm{~d} 7 \mathrm{~s}^{2}$. Based on the lawrencium results, it was proposed that rutherfordium should also contain an occupied stabilized $7 p$ orbital [KEL84], and should have the electronic structure 
$[R n] 5 f^{147} 7 s^{2} 7 p^{2}$ rather than the $[R n] 5 f^{14} 6 d^{2} 7 s^{2}$ structure analogous to the electronic structure of hafnium $[X e] 5 f^{14} 6 d^{2} 7 s^{2}$.

However, recent MCDF relativistic calculations [GLE89] using $468 \mathrm{j}-\mathrm{j}$ couplings indicate that the ground state of the neutral atom should be $[R n] 5 f^{14} 6 d 7 s^{2} 7 p(80 \%)$ with a $[R n] 5 f^{14} 6 d^{2} 7 s^{2}$ state $0.5 \mathrm{eV}$ above the ground state. The $[R n] 5 f^{14} 7 s^{2} 7 p^{2}$ electronic configuration is calculated to be $2.9 \mathrm{eV}$ above the ground state. Other MCDF calculations also support $[R n] 5 f^{14} 6 \mathrm{~d} 7 \mathrm{~s}^{2} 7 \mathrm{p}$ as the ground state electronic configuration for rutherfordium [JOH9O].

\section{B. History}

Soviet and American researchers have come to an impasse concerning the resolution of the priority of discovery of the transactinide elements with $Z=104$ and 105 [HYD87]. This conflict stems from differing claims by scientists from the Joint Institute for Nuclear Research at Dubna in the Soviet Union and the Lawrence Berkeley Laboratory (LBL) in the United States. Each group has claimed discovery of elements 104 and 105 and has proposed different names.

For element 104, the Soviet discoverers [FLE64] proposed the name kurchatovium (Ku), and claimed to have produced a $300-\mathrm{ms} 260 \mathrm{Ku}$, which decays by spontaneous fission. The half-life was later reduced to $80 \mathrm{~ms}$ and then $20 \mathrm{~ms}$ [HYD87]. They claimed it was produced via a ${ }^{244} \mathrm{Pu}\left({ }^{22} \mathrm{Ne}, 6 \mathrm{n}\right)$ reaction. The Berkeley discovery group [GHI69] proposed the name used in this paper, rutherfordium. The Berkeley team claimed to have produced ${ }^{257} \mathrm{Rf}$ from the reaction of ${ }^{12} \mathrm{C}$ with ${ }^{249} \mathrm{Cf}$. The claim to priority of discovery is still in dispute, over 20 years after the fact. IUPAC has sought to solve the naming problem by introducing a contrived naming system to be used for the transactinide elements until discovery is assigned and names are approved. The system, however, is unlikely to be implemented by any transactinide 
research team. Currently, papers submitted by the Dubna team use the symbol $\mathrm{Ku}$ while the LBL and related researchers utilize $\mathrm{Rf}$ as the symbol for element 104. Experience has dictated that when the Dubna and LBL researchers meet, the symbols are dropped and "element 104" is used.

The first chemical studies to be done on the new element rutherfordium were in the gas phase. In 1966 the volatility of Group 4 tetrachlorides was studied with a gas chromatographic technique [ZVA67]. In this method, the rutherfordium and other reaction product atoms were chlorinated with $\mathrm{TiCl}_{4}$ or $\mathrm{SOCl}_{2}$ prior to entering a chromatographic column. A temperature gradient was established in a section of the column. The gradient section was lined with mica track detectors to detect SF fragments. A 0.3-S SF activity, assumed to be $260 \mathrm{Ku}$ produced via the $242 \mathrm{Pu}(22 \mathrm{Ne}, 4 \mathrm{n})$ reaction, was found to behave similarly to hafnium and zirconium and deposit in the same section of the column, while the actinides deposited earlier. This experiment showed the similar volatilities of the chloride species of zirconium, hafnium, and rutherfordium, indicating they have similar chemical properties and belong in the same element group [ZVA67], [ZVA7O].

However, the 1966 chromatography experiments could not have detected an 80-ms SF activity as the column used in the experiments had a 1.2 second transit time for the volatile chlorides. In retrospect, Zvara and coworkers [HYD87] later claimed these experiments actually measured 3-s $259 \mathrm{Ku}$, which would have survived the transit through the thermochromatography column. This revision is not consistent with the originai description of the experiments where it was stated "positively that the half-life could not be $3.7 \mathrm{~s}$ " [SEA90]. Because of such inconsistencies, many regard these experiments as not providing satisfactory evidence for the discovery of element 104 [HYD87]. 
Solution chemistry on rutherfordium was first performed in 1970 after the discovery of ${ }^{261} \mathrm{Rf}$ [SIL70]. The isotope ${ }^{261} \mathrm{Rf}\left(T_{1 / 2}=65 \mathrm{~s}\right)$ was first produced at Berkeley by the ${ }^{248} \mathrm{Cm}\left({ }^{18} \mathrm{O}, 5 \mathrm{n}\right)$ reaction [GHI70]. In the initial solution chemistry experiments, ${ }^{261} \mathrm{Rf}$ was eluted from a cation exchange resin column with $0.1 \mathrm{M}$ ammonium $\alpha$-hydroxyisobutyrate at $\mathrm{pH} 4$, together with zirconium and hafnium [SIL70]. The results showed that rutherfordium acted chemically similar to the Group 4 elements zirconium and hafnium, by forming species not absorbing on the cation exchange resin, while the trivalent actinides sorb on the cation exchange resin under these conditions. The formation of anionic rutherfordium chloride complexes was studied in additional experiments [HUL80]. The chloride complexes of hatnium, curium, fermium, and rutherfordium were tested for their relative absorption on a column containing quaternary amines held on an inert support. The results showed that rutherfordium and hafnium formed similar anionic chloride species in $12 \mathrm{M} \mathrm{HCl}$, chemically unlike the trivalent actinides which did not. 


\section{Chapter 2: Experimental Procedures}

\section{A. Cyclotron-Produced Isotopes}

\section{Target System}

All irradiations were carried out at the LBL 88-Inch Cyclotron. A schematic of the target system is shown in Figure 2. The beam enters the target system through a $6-\mathrm{mm}$ collimator, which defines the diameter of the beam entering the target. Next, the beam passes through a Havar vacuum isolation window $\left(1.8 \mathrm{mg} / \mathrm{cm}^{2}\right)$, which separates the target system from the cyclotron vacuum. The beam passes through the nitrogen gas $\left(0.2 \mathrm{mg} / \mathrm{cm}^{2}\right)$, used to cool the target and then strikes the $B e$ target backing $\left(2.75 \mathrm{mg} / \mathrm{cm}^{2}\right)$ and finally enters the target material. The beam is stopped in a graphite block mounted on the back plate of the target chamber. The beam stop and the collimator are water cooled to prevent heat build up due to the beam. The beam energies incident on the target were calculated from range tables [NOR70], [HUB90].

\section{Target Preparation}

All the various targets were prepared in the same manner. Chemical purification of some of the target materials was done prior to target preparation. The target material was converted to the nitrate form by evaporation in $2 \mathrm{M}$ $\mathrm{HNO}_{3}$. The nitrate salt was dissolved in $2 \mathrm{~mL}$ of isopropanol. The target material was electrodeposited in $50 \mu \mathrm{g} / \mathrm{cm}^{2}$ layers onto a $1 \mathrm{mil}\left(2.75 \mathrm{mg} / \mathrm{cm}^{2}\right)$ thick Be foil. The target diameter is $6 \mathrm{~mm}$, the diameter of the electroplating chimney. The deposition was carried out by applying a voltage of several hundred volts and a current less than $6 \mathrm{~mA}$ [AUM74], [EVA72], [MUL75]. The isopropanol solution was stirred by attaching the plating electrode to the center of a small speaker. The vibrating speaker mixed the solution.

After deposition of each nitrate layer, the larget was dried under a heat lamp and baked in an oven at $450.500^{\circ} \mathrm{C}$ for 30 minutes to convert the deposit to an 
oxide. The deposition was continued until the desired target thickness was achieved. The target thickness was determined by measuring the decay of the target material or an added $241 \mathrm{Am}$ tracer.

Figure 2. Target System

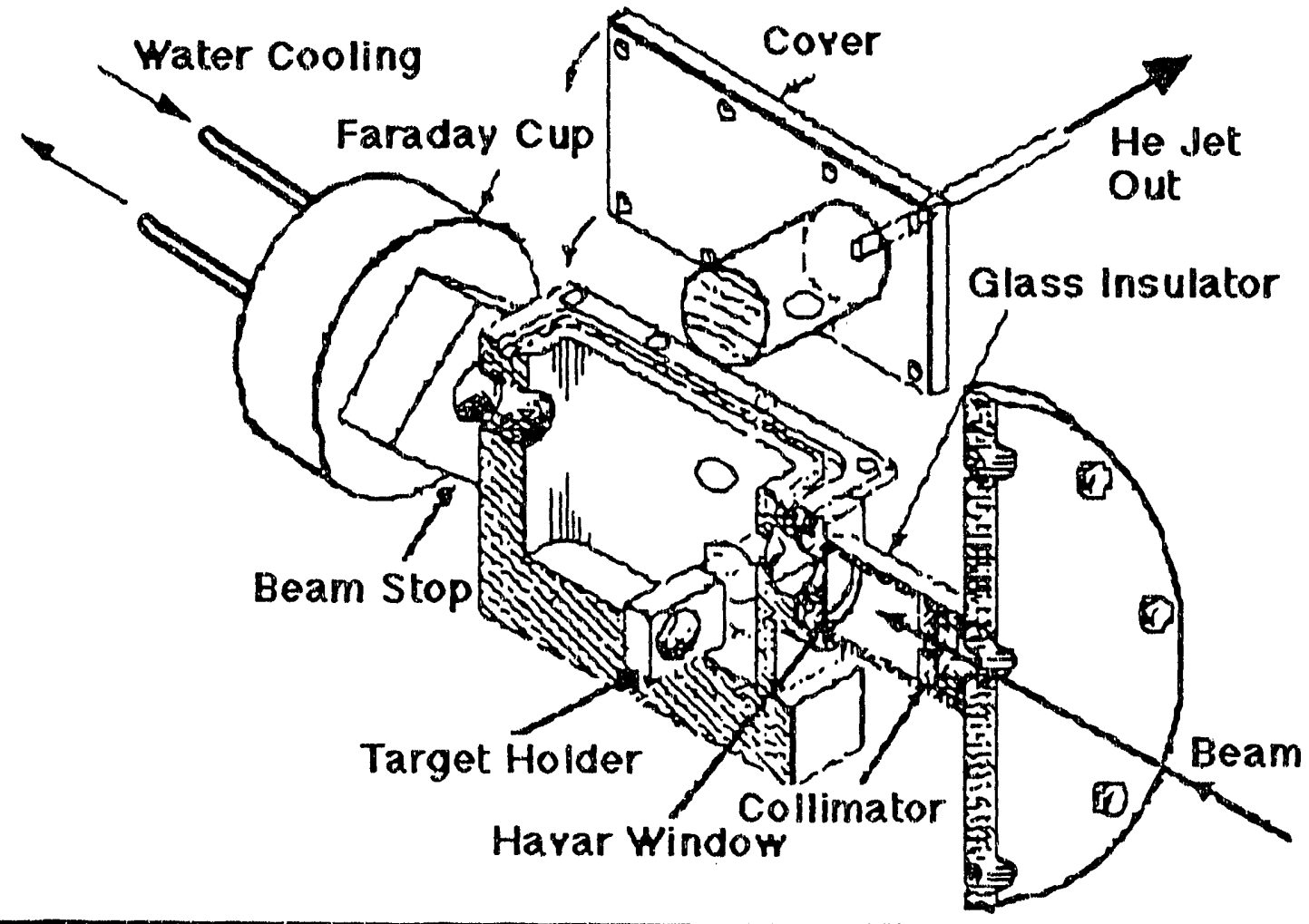

3. Gas-Jet Transport System

The recoiling product nuclei were transported to the chemistry collection site via a helium $\mathrm{KCl}$ gas-jet transport system. The $\mathrm{KCl}$ aerosols were generated by heating crystalline $\mathrm{KCl}$ in a quartz tube over which helium gas was passed. The $\mathrm{KCl}$ clusters were swept out of the quartz tube by the helium and moved into a presorter capillary, where the larger $\mathrm{KCl}$ aerosols were removed from the heliurn gas flow. The presorter capillary is a 5 meter length of $2.2 \mathrm{~mm}$ diameter polyvinyl chloride tubing wound into several coils of $15 \mathrm{~cm}$ diameter. 
The aerosols were then transported to the target chamber via a $1 \mathrm{~cm}$ diameter tygon tube. The aerosols were restricted to a small area behind the target where the recoils attached to the aerosols. They were then transported via 7 meters of polyvinyl chloride tubing to the collection site in a hood. The entrance to this polyvinyl chloride transport tube inside the target system is $2 \mathrm{~cm}$ behind the target.

The chemistry collection site was inside a small lucite box in the corner of a hood near the cave where the irradiations are conducted (the Cave 0 mezzanine chemistry hood at the 88-Inch Cyclotron). The aerosnis containing recoil nuclei were collected under vacuum from the end of the capillary on a teflon square. There were four rotating collection sites on the periphery of a 30 $\mathrm{cm}$ diameter circular brass plate. The collection sites were rotated by disengaging the vacuum with an electric solenoid valve. A SF/alpha detector was placed at the collection site to monitor any alpha or SF activity which would indicate a target rupture. The collection site, with the product nuclei and the collected $\mathrm{KCl}$ aerosols, could be moved in front of the chemist to perform experiments. Collection time and collection site movement were manually controlled by the chemist. Fast chemistry was performed in the Cave 0 mezzanine hood, and samples were made for alpha or gamma spectroscopy as described.

Studies were conducted to determine optimum conditions for maximizing the yield of the gas-jet transport system. The variables studied were the polyvinyl chloride tube diameter from the target chamber to the collection site $(2.2 \mathrm{~mm}$ or $1.2 \mathrm{~mm}$ ) and the oven temperature. The ability to vary the He gas pressure is restricted. The He gas pressure at the target needs to be 3 psi, since the nitrogen cooling gas is also 3 psi. A significant difference in the two gas pressures would strain the target backing and might cause it to rupture. 
The gas-jet yield was studied using $213 \mathrm{Bi}$ recoiling from a $225 \mathrm{Ac}\left(3 \times 10^{4}\right.$ $\mathrm{dpm}$ ) source (Figure 3). The transport of Bi by the gas-jet should not differ significantly from the transport of the recoil nuclei from the reactions being studied. The recoil source simulated the product nuclei recoiling from a nuclear reaction, and was in the $\mathrm{He} / \mathrm{KCl}$ gas-jet flow between the $\mathrm{KCl}$ oven and the chemistry collection site. The $213 \mathrm{Bi}$ was monitored by its $5.87 \mathrm{MeV}$ alpha activity. A direct catch of the $213 \mathrm{Bi}$ was done to determine $100 \%$ transport. The direct catch was accomplished by placing a tantalum foil $1 \mathrm{~cm}$ in front of the recoil source and collecting for 5 minutes. The activity on the foil was counted and a $100 \%$ decay rate was determined. The gas-jet oven temperature and capillary diameter from the recoil site to the chemistry collection site was varied. A series of five minute collections were conducted for each experiment.

The first variable examined was the capillary diameter from the recoil source to the collection site. The capillary is polyvinyl chloride and was often changed between cyclotron experiments. Two sizes of capillaries were studied, one having an inner diameter of $2.2 \mathrm{~mm}$, the other with a $1.2 \mathrm{~mm}$ inner diameter. The larger capillary was used for gas chemistry studies; the smaller capillary was employed during aqueous chemistry studies. The results, presented in Table 2, show that the $1.2 \mathrm{~mm}$ inner diameter capillary gives a larger yield. The temperature listed is the temperature of the gas-jet oven. The gas flow is given in liters per minute.

The reason for the higher ${ }^{213} \mathrm{Bi}$ yield with the smaller capillary is due to the lower gas flow rate associated with the smaller capillary. The $\mathrm{KCl}$ spot, which contains the recoil activity, increased with capillary size. Inspection showed that the larger $\mathrm{KCl}$ spot tends to create $\mathrm{KCl}$ flakes which can be removed from the collection site by the vacuum. 


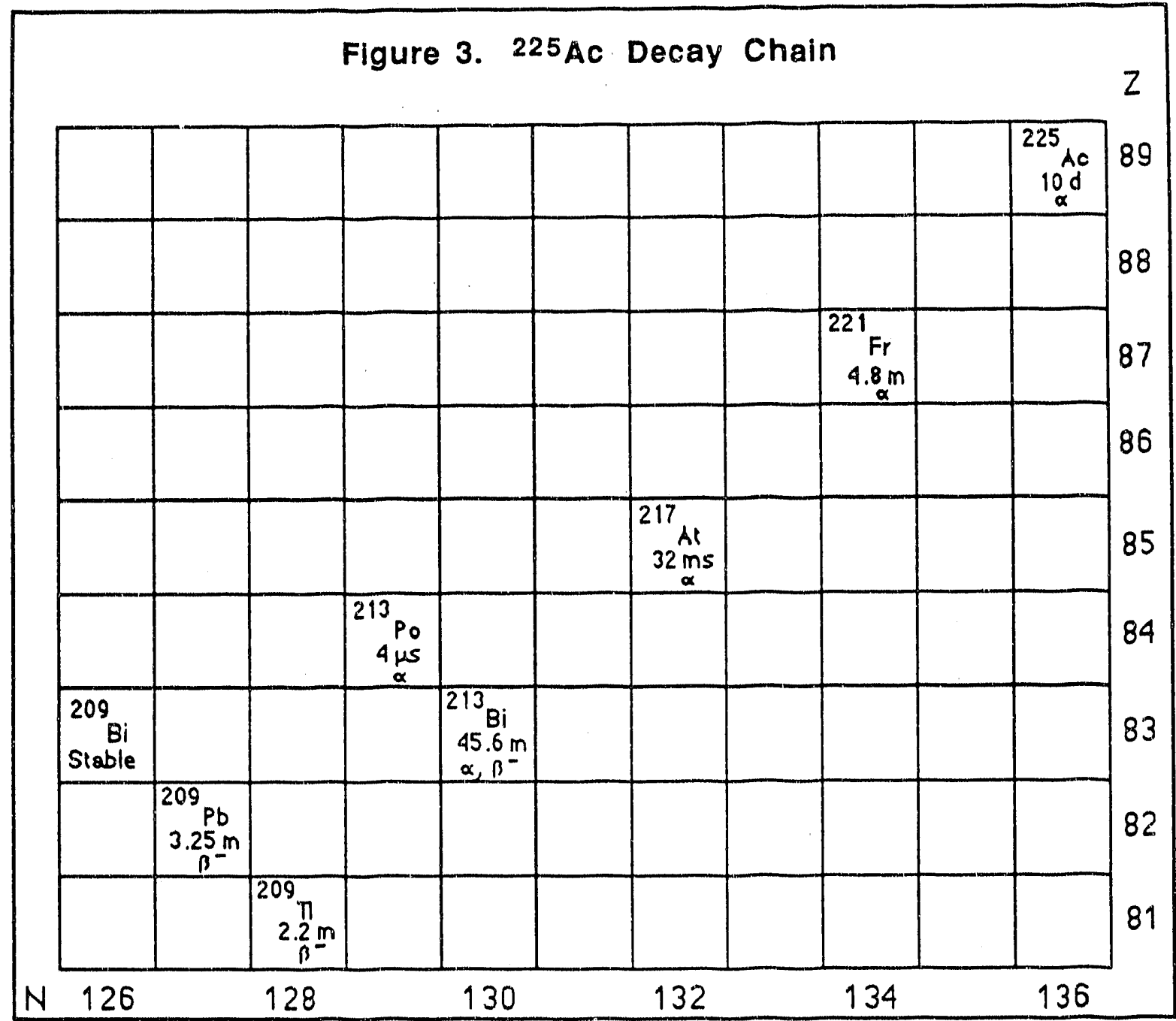

Table 2. Effect of Capillary Size on Gas-Jet Yield

\begin{tabular}{llll}
$\begin{array}{l}\text { Capillary Size } \\
(\mathrm{mm})\end{array}$ & $\begin{array}{l}\text { Temp. } \\
\left({ }^{\circ} \mathrm{C}\right)\end{array}$ & $\begin{array}{l}\text { Gas Flow } \\
(\mathrm{L} / \mathrm{m})\end{array}$ & $\begin{array}{l}\text { Yield } \\
(\%)\end{array}$ \\
1.2 & 670 & 2.92 & $88.6 \pm 1.6$ \\
2.2 & 670 & 6.40 & $41.1 \pm 1.1$ \\
\hline
\end{tabular}

An experiment in which the gas flow in the $1.2-\mathrm{mm}$ capillary was varied shows that an increase in gas flow decreases the gas transport yield. This is due to the increased $\mathrm{KCl}$ flaking associated with a thicker $\mathrm{KCl}$ spot. The amount of $\mathrm{KCl}$ deposited is proportional to the gas flow. The results from these 
experiments are presented in Table 3. The pressure, in pounds per square inch, would be the pressure on the target from the $\mathrm{He} / \mathrm{KCl}$ aerosol gas-jet.

The results of the experiments indicated that the use of $1.2 \mathrm{~mm}$ diameter polyvinyl chloride tubing for aerosol transport from the recoil chamber to the chemistry collection site increased the gas transport yield. Additionally, little yield is lost by holding the gas pressure to 3 psi on the target.

\begin{tabular}{|llll|}
\hline & Table 3. Effect of Gas Flow on Transport Yield \\
Temp & $\begin{array}{l}\text { Gas Flow } \\
(\mathrm{L} / \mathrm{m})\end{array}$ & $\begin{array}{l}\text { Pressure } \\
(\mathrm{PS})\end{array}$ & $\begin{array}{l}\text { Yield } \\
(\%)\end{array}$ \\
670 & 2.92 & 3 & $88.6 \pm 1.6$ \\
670 & 3.46 & 4.5 & $48.1 \pm 1.2$ \\
670 & 4.00 & 6 & $38.3 \pm 1.1$ \\
\hline
\end{tabular}

To further maximize the gas-jet yield, the effects of oven temperature were studied. The amount of $\mathrm{KCl}$ in the aerosol increases as the oven temperature increases. Figure 4 shows the effect of oven temperature on gas-jet yield. The gas-jet yield reached a maximum at $650^{\circ} \mathrm{C}$, with a gas-jet yield of $95.7 \%$. From the experiments, it was found the gas-jet yield was maximized using an oven temperature of $650^{\circ} \mathrm{C}$ and a $1.2 \mathrm{~mm}$ polyvinyl chloride capillary to transport the $\mathrm{KCl}$ aerosols from the target chamber to the chemistry collection site. Under these conditions, the He gas flow was $\approx 3 \mathrm{~L} / \mathrm{m}$ and the gas pressure on the target from the He was $3 \mathrm{psi}$. These gas-jet conditions were used to transport the recoil nuclei in all cyclotron experiments.

The mass of $\mathrm{KCl}$ deposited as a function of time was determined by titration with excess $\mathrm{AgNO}_{3}$ and $\mathrm{NaSCN}$. Iron in the form of $\mathrm{Fe}\left(\mathrm{NO}_{3}\right)_{3}$ was added as an 
indicator. Iron forms a red $\mathrm{Fe}(\mathrm{SCN})_{3}$ complex at the titration end point. The chloride from the $\mathrm{KCl}$ forms $\mathrm{AgCl}$. The excess silver forms AgSCN. The SCNanion preferentially complexes the $\mathrm{Ag}$, and the $\mathrm{Fe}(\mathrm{SCN})_{3}$ complex forms after all the silver is complexed, thus determining the end point [YOU53].

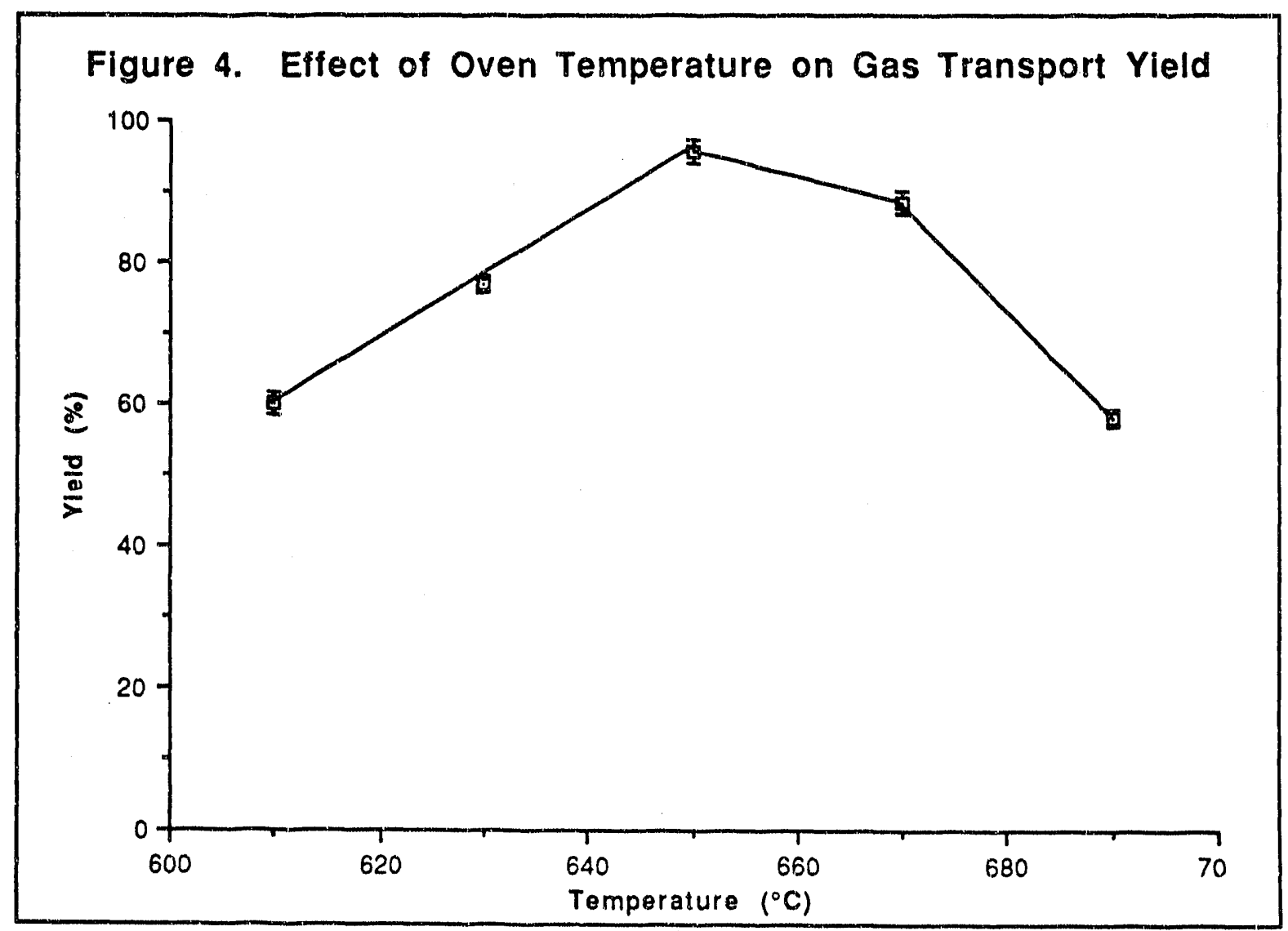

The mass of $\mathrm{KCl}$ was determined for the conditions of maximum gas transport yield: an oven temperature of $650^{\circ} \mathrm{C}$; a $1.2 \mathrm{~mm}$ polyvinyl chloride capillary; He gas flow $\approx 3 \mathrm{~L} / \mathrm{m}$; and the He gas pressure on the target of 3 psi. The $\mathrm{KCl}$ was allowed to collect on glass cover slips for 10 minutes. The cover slips were washed three times with $50 \mu \mathrm{L}$ of water. The washings were transferred to a $1 \mathrm{~mL}$ glass centrifuge cone and $50 \mu \mathrm{L}$ of $0.9997 \mathrm{M} \mathrm{AgNO}_{3}$ and excess $\mathrm{Fe}\left(\mathrm{NO}_{3}\right)_{3}$ were added. A $1.001 \mathrm{M} \mathrm{NaSCN}$ solution was added in $2 \mu \mathrm{L}$ increments and mixed. The NaSCN solution was added until the end point was 
reached. The results showed $308.3 \pm 25.1 \mu \mathrm{g}$ of $\mathrm{KCl}$ was deposited during the 10 minute collection.

\section{Isotope Production}

\section{a. $261 \mathrm{Rf}$}

The isotope $261 \mathrm{Rf}$ was produced via the $248 \mathrm{Cm}\left({ }^{18} \mathrm{O}^{5+}, 5 \mathrm{n}\right)$ reaction. The reaction has a cross section of $5 \mathrm{nb}$ with $98 \mathrm{MeV} 180$ ions [GHI70]. The ${ }^{180}$ beam intensity averaged 0.5 particle microamp $(\mathrm{P} \mu \mathrm{A})$. The $261 \mathrm{Rf}$ prortuction rate with this beam intensity and target was 0.9 atom $/$ minute. The target material, ${ }^{248} \mathrm{Cm}_{2} \mathrm{O}_{3}$, was prepared by the electrodeposition of $248 \mathrm{Cm}\left(\mathrm{NO}_{3}\right)_{3}$ on Be foil using the molecular deposition technique as described in Section 2.A.2.

The $248 \mathrm{Cm}$ was separated from its $244 \mathrm{Pu}$ daughter by elution from an anion exchange column with $12 \mathrm{M} \mathrm{HCl}$. The $12 \mathrm{M} \mathrm{HCl}$ eluant, containing the purified ${ }^{248} \mathrm{Cm}$, was taken to dryness under a heat lamp. The target thickness was determined by measuring the $5.078-\mathrm{MeV}$ alpha of the $248 \mathrm{Cm}$ target material. The final thickness of the $248 \mathrm{Cm}$ used for the production of $261 \mathrm{Rf}$ was 0.74 $\mathrm{mg} / \mathrm{cm}^{2}$.

\section{b. $162 \mathrm{Hf}$}

Hatnium is the third row member of the Group 4 elements. As a Group 4 element, it is a homolog of rutherfordium. The chemistry of hafnium was studied as a model for the chemistry of ruthertordium. Extrapolation from the chemistry of hafnium can be used to infer the chemical properties of rutherfordium.

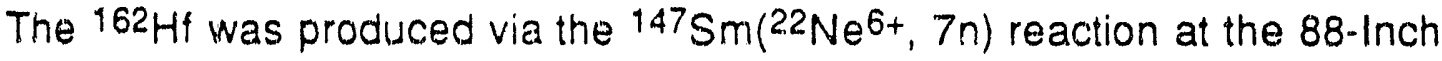
cyclotron for exiraction studies with 2-thenoyltrifluoroacetone (TTA). The target material, ${ }^{147} \mathrm{Sm}_{2} \mathrm{O}_{3}\left(0.26 \mathrm{mg} / \mathrm{cm}^{2}\right)$, was made by the electrodeposition technique as described in Section 2.A.2. The reaction cross section is $10 \mathrm{mb}$ for $140 . \mathrm{MeV} 22 \mathrm{Ne}$ ions. The average beam intensity was $0.5 \mathrm{p \mu A}$. 
The $147 \mathrm{Sm}$ starting material was the oxide, which was converted to the nitrate in concentrated nitric acid. The solution was evaporated and dissolved in isopropanol. Tracer ${ }^{241} \mathrm{Am}$ was added to determine the target thickness. The final target was electroplated as described in Section 2.A.2. The final $147 \mathrm{Sm}$ target thickness was $0.26 \mathrm{mg} / \mathrm{cm}^{2}$. The $162 \mathrm{Hf}$ photopeaks were measured using a GeLi spectrometer system. All gamma photopeak measurements for the $162 \mathrm{Hf}$ were done at the 88 -Inch Cyclotron immediately following separation. The $162 \mathrm{Hf}$ decay information is given in Table 4 .

\begin{tabular}{|c|c|c|c|c|c|}
\hline \multirow[b]{2}{*}{ Half-Life } & \multicolumn{5}{|c|}{ Table 4. $162 \mathrm{Hf}$ Decay Information } \\
\hline & Decay & Mode & $\begin{array}{l}\gamma \text {-Ray } \\
(k \in V)\end{array}$ & Energy & $\begin{array}{l}\text { Intensity } \\
(\%)\end{array}$ \\
\hline \multirow[t]{2}{*}{$37.6 \mathrm{~s}$} & $E C, B^{+}$ & & 173.9 & & 100 \\
\hline & & & 196.3 & & 25 \\
\hline
\end{tabular}

\section{c. $169 \mathrm{HI}$}

The isotope ${ }^{169} \mathrm{Hf}$ was produced via the nat $\mathrm{Gd}\left(18 \mathrm{O}^{5+}+\mathrm{xn}\right)$ reaction, with $\mathrm{x}=$ 4-7, for extraction studies with tributylphosphate (TBP). The target material, nat $\mathrm{Gd}_{2} \mathrm{O}_{3}$, was made by the electrodeposition technique from an isopropanol solution [AUM74], [EVA72], [MUL75], as described previously.

The starting material was $\mathrm{Gd}_{2} \mathrm{O}_{3}$, which was converted to the nitrate in concentrated nitric acid. The solution was evaporated and dissolved in isopropanol. Tracer ${ }^{241} \mathrm{Am}$ was added to determine the target thickness. The final natGd target thickness was $500 \mu \mathrm{g} / \mathrm{cm}^{2}$. The reaction cross section was 10 $\mathrm{mb}$ with $98 \mathrm{MeV} 180$ ions. The average beam intensity was $0.5 \mathrm{p \mu A}$. The same 180 beam energy was used as in the 261 Rf production reaction so the cyclotron beam energy would not have to be changed. The ${ }^{169} \mathrm{Hf}$ decay information is 
given in Table 5. The ${ }^{169} \mathrm{Hf}$ photopeaks were measured with a GeLi spectrometer system.

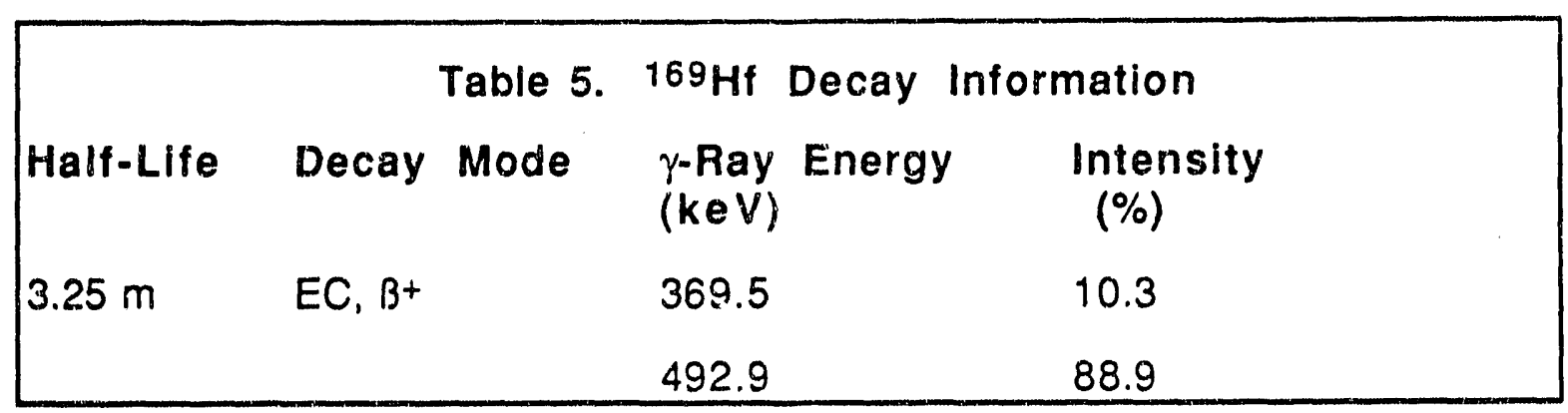

\section{B. Tracer Solutions}

All tracers were stored in acid solutions in polypropylene centrifuge cones. The acidity of these solutions was kept as high as allowed by the experiments to be conducted (usually $>6 \mathrm{M}$ ) in order to prevent hydrolysis. The tetravalent metals are prone to hydrolysis and may polymerize during long storage in low acid concentrations. The solutions were stored in polypropylene rather than glass since oxidized tetravalent metal ions in solution can adhere to glass. The acid concentravion of the final experimental stock solutions was adjusted by evaporating the solution to dryness and redissolving the radiotracer in a solution of the desired acid concentration. The specific activities of the tracer solutions varied with the isotope. Total solution volumes were usually $500 \mu \mathrm{L}$ each of the organic and aqueous phases. The decay information for the tracers is listed in Tables 6 and 7. The electronic structures for the neutral atoms and ionic radii for various coordination numbers and oxidation states are listed in Table 8. 


\begin{tabular}{|c|c|c|c|c|}
\hline \multirow[b]{2}{*}{ Isotope; } & \multicolumn{4}{|c|}{ Table 6. $95 \mathrm{Zr}, 95 \mathrm{Nb}$, and $152 \mathrm{Eu}$ Decay Information } \\
\hline & Half-Life & Decay Mode & $\begin{array}{l}\gamma \text {-Ray Energy } \\
(\mathrm{keV})\end{array}$ & $\begin{array}{l}\text { Intensity } \\
(\%)\end{array}$ \\
\hline \multirow[t]{2}{*}{$95 \mathrm{Zr}$} & $64.02 d$ & B- & 756.7 & 55.4 \\
\hline & & & 724.2 & 43.7 \\
\hline $95 \mathrm{Nb}$ & $34.97 \mathrm{~d}$ & B- & 765.8 & 99.9 \\
\hline \multirow[t]{2}{*}{${ }^{144} \mathrm{Ce}$} & $284.6 d$ & B- & 80.1 & 1.7 \\
\hline & & & 133.5 & 10.8 \\
\hline \multirow[t]{7}{*}{ 152EU } & $13.3 y$ & $E C, B^{+}, B^{-}$ & 121.8 & 28.4 \\
\hline & & & 344.3 & 26.6 \\
\hline & & & 778.9 & 13.0 \\
\hline & & & 964.1 & 14.6 \\
\hline & & & 1058.9 & 10.2 \\
\hline & & & 1112.1 & 13.6 \\
\hline & & & 1408.0 & 20.8 \\
\hline \multirow[t]{2}{*}{$172 \mathrm{Hf}$} & $1.87 \mathrm{y}$ & $\mathrm{EC}$ & 24.0 & 20.4 \\
\hline & & & 125.9 & 9.1 \\
\hline
\end{tabular}

Table 7. 228Th and 238Pu Decay Information

\begin{tabular}{|lllll} 
Isotope & $\begin{array}{l}\text { Half-Life } \\
\text { (years) }\end{array}$ & Decay Mode & $\begin{array}{l}\alpha \text {-Particle Energy } \\
\text { (MeV) }\end{array}$ & $\begin{array}{l}\text { Intensity } \\
(\%)\end{array}$ \\
$228 T h$ & 1.91 & $\alpha$ & 5.34 & 26.7 \\
& & & 5.42 & 72.7 \\
$238 \mathrm{Pu}$ & 87.7 & $\alpha$ & 5.45 & 28.7 \\
& & & 5.50 & 71.7 \\
\hline
\end{tabular}

Reference [LED78] 
Table 8. Neutral Atom Electronic Structure and lonic Radius

\begin{tabular}{|c|c|c|c|c|}
\hline Element & $\begin{array}{l}\text { Electronic } \\
\text { Structure }\end{array}$ & $\begin{array}{l}\text { Oxidation } \\
\text { States }\end{array}$ & $\begin{array}{l}\text { Coordination } \\
\text { Number }\end{array}$ & $\begin{array}{l}\text { Ionic } \\
\text { Radius } \\
(\mathrm{pm})\end{array}$ \\
\hline \multirow[t]{3}{*}{$Z r$} & {$[K r] 4 d^{2} 5 s^{2}$} & $4^{+}$ & 4 & 59 \\
\hline & & & 6 & 72 \\
\hline & & & 8 & 84 \\
\hline \multirow[t]{2}{*}{$\mathrm{Ce}$} & {$[X e] 4 f^{1} 5 d^{1} 6 s^{1}$} & $4^{+}$ & 6 & 87 \\
\hline & & & 8 & 97 \\
\hline Eu & {$[X e] 4 \uparrow^{7} 6 s^{2}$} & $3^{+}$ & 6 & 95 \\
\hline \multirow[t]{3}{*}{$\mathrm{Hf}$} & {$[X e] 4 f^{14} 5 d^{2} 6 s^{2}$} & $4^{+}$ & 4 & 58 \\
\hline & & & 6 & 71 \\
\hline & & & 8 & 83 \\
\hline \multirow[t]{2}{*}{ Th } & {$[R n] 6 d^{2} 7 s^{2}$} & $4^{+}$ & 6 & 94 \\
\hline & & & 8 & 105 \\
\hline \multirow[t]{2}{*}{$\mathrm{Pu}$} & {$[R n] 5 f^{6} 7 s^{2}$} & $4^{+}$ & 6 & 86 \\
\hline & & & 8 & 96 \\
\hline
\end{tabular}

Reference [SHA76]

1. $95 \mathrm{Zr}$

Zirconium, the second row Group 4 element, is a lighter homolog of rutherfordium since it is a member of the same group. As with hafnium, the chemistry of zirconium was studied as a model for the chemistry of rutherfordium. A major difference between zirconium and the heavier Group 4 elements is that zirconium lacks occupied f-orbitals.

The daughter of $95 \mathrm{Zr}, 95 \mathrm{Nb}$, was also studied during the zirconium extraction experiments. The $95 \mathrm{Nb}$ was not purified from the $95 \mathrm{Zr}$ and was present in all tracer solutions. Some zirconium chemical separations will also separate 
niobium. Niobium, a Group 5 element, is a hahnium (element 105) homolog. The $95 \mathrm{Zr}$ was used in the extraction studies of trialkylamines, TBP and TTA.

The isotope $95 \mathrm{Zr}$ is a fission product and was obtained from a variety of sources. New England Nuclear provided the initial $95 \mathrm{Zr}$ samples in oxalic acid solutions. This solution was evaporated and redissolved in $10 \mathrm{M} \mathrm{HCl}$ solution and stored in a polypropylene centrifuge cone.

The isotope $95 \mathrm{Zr}$ was also made by fission of uranium utilizing neutrons produced during proton bombardments at the 88-Inch Cyclotron. Samples of natural uranyl nitrate were enclosed in polypropylene centrifuge cones and mounted behind the beam stop outside the target chamber. The secondary neutrons produced by the light ion reaction caused fission of the uranium and $95 \mathrm{Zr}$ was separated from the fission products. The uranyl nitrate was dissolved in $12 \mathrm{M} \mathrm{HCl}$, and the $95 \mathrm{Zr}$ was extracted into $1.0 \mathrm{M}$ triisooctylamine (TIOA) in benzene. The TIOA was then washed with $4 \mathrm{M} \mathrm{HCl}$ to remove the $95 \mathrm{Zr}$. The acid solution containing the $95 \mathrm{Zr}$ was evaporated and redissolved in enough $12 \mathrm{M} \mathrm{HCl}$ so the specific activity of the solution was $10 \mathrm{nCi} / 50 \mu \mathrm{L}$. A total of 400 nCi of $95 \mathrm{Zr}$ was produced via this method, enough for 40 extraction experiments.

Samples of $95 \mathrm{Zr}$ were also obtained from Los Alamos National Laboratory in the form of isotopically pure $95 \mathrm{Zr}$ embedded in aluminum foil during isotopic separation. The zirconium had been chemically separated from the other fission products prior to the isotope separation. The $95 \mathrm{Zr}$ was separated from the aluminum foil by dissolving the portion of the foil with the $95 \mathrm{Zr}$ in $12 \mathrm{M} \mathrm{HCl}$. The $95 \mathrm{Zr}$ was extracted into $0.25 \mathrm{M}$ TBP in benzene, leaving the aluminum in the aqueous phase. The TBP solution was washed with $4 \mathrm{M} \mathrm{HCl}$ to remove the $95 \mathrm{Zr}$ from the organic phase. The acid solution was evaporated to dryness and the $95 \mathrm{Zr}$ redissolved in enough $12 \mathrm{M} \mathrm{HCl}$ to make a soiution with a specific 
activity of $10 \mathrm{nCi} / 50 \mu \mathrm{L}$. The amount of $95 \mathrm{Zr}$ activity isolated from the foil was about $320 \mathrm{nCi}$.

\section{2. ${ }^{144 \mathrm{Ce}}$}

Cerium is the first member of the lanthanide series. Cerium, like the other lanthanides, has a $3^{+}$oxidation state. However, under basic conditions, the $4^{+}$ oxidation state predominates due to stabilization by the dioxide state [GRE84]. Cerium extraction was studied with the synthesized LICAM (LInear Catecholate AMide) ligands in basic solutions.

The isotope ${ }^{144} \mathrm{Ce}$ is a fission product, and was purchased from Isotope Products (Burbank, CA) in $6 \mathrm{M} \mathrm{HCl}$ solution. The ${ }^{144} \mathrm{Ce}$ solution was evaporated and redissolved in $0.2 \mathrm{M} \mathrm{HCl}$ with no further purification. Enough $0.2 \mathrm{M} \mathrm{HCl}$ was added to give a stock solution with a specific activity of 360 $\mathrm{nCi} / 30 \mu \mathrm{L}$. The ${ }^{144} \mathrm{Ce}$ was measured by gamma spectroscopy.

3. $152 \mathrm{E} \mathrm{U}$

Europium is a member of the lanthanide series and was used as a trivalent actinide homolog. It was important to study the chemistry of the trivalent lanthanides to determine if the separations would be free of trivalent actinides. Europium has a stable $3^{+}$oxidation state, but can be reduced to the divalent state. No europium reduction was conducted and only the trivalent state was used in these experiments.

The isotope ${ }^{152} \mathrm{Eu}$ is a fission product and was purchased from Isotope Products in $4 \mathrm{M} \mathrm{HCl}$. The ${ }^{152} \mathrm{Eu}$ was used in the LICAM experiments and the trialkylamine extractions. The stock solutions were made by evaporation of the original solution and redissolving the $152 \mathrm{Eu}$ in as concentrated $\mathrm{HCl}$ solution as possible without any chemical purification of the resulting sample. The resulting 152Eu stock solutions had a specific activity of $36 \mathrm{nCi} / 30 \mu \mathrm{L}$. The 152Eu was measured by gamma spectroscopy. 


\section{4. $172 \mathrm{Hf}$}

The isotope $172 \mathrm{Hf}$ is a spallation product of high energy protons on bismuth and was obtained from Isotope Products in Burbank, CA. The original storage solution was $6 \mathrm{M} \mathrm{HCl}$. The stock solutions were made by evaporation of the original solution and redissolving the $172 \mathrm{Hf}$ in $12 \mathrm{M} \mathrm{HCl}$ solution without any chemical purification of the resulting sample. The resulting $172 \mathrm{Hf}$ stock solutions had a specific activity of $10 \mathrm{nCi} / 50 \mu \mathrm{L}$. The $172 \mathrm{Hf}$ was measured by gamma spectroscopy and was used in the TTA extraction studies.

\section{5. $228 \mathrm{Th}$}

Thorium is the first member of the actinide series. Unlike the lanthanides, some members of the actinide elements have stable tetravalent states, of which thorium is an example. It exhibits only the $4^{+}$oxidation state in aqueous solution. Thorium is not a true rutherfordium homolog, since it is not a member of the Group 4 series. However, since thorium has a tetravalent oxidation state like rutherfordium, it may have similar chemical properties. The use of tetravalent actinides for chemical comparison to rutherfordium was initiated since it has been shown that under certain conditions, protactinium behaves similarly to hahnium [GOB91], [GRE91], [KRA89]. The pentavalent oxidation state is the stable oxidation state for both protactinium and hahnium.

The isotope $228 \mathrm{Th}$ is a naturally occurring radioactive isotope of the $232 \mathrm{Th}$ chain. A 228Th stock solution was prepared in $6 \mathrm{M} \mathrm{HCl}$. The 228Th stock solutions were evaporated and redissolved in as concentrated $\mathrm{HCO}$ solution as possible. Enough $\mathrm{HCl}$ was added to give a specific activity of $360 \mathrm{nCi} / 30 \mu \mathrm{L}$. The 228 Th was used in the diethylenetriaminepentaacetic acid (DTPA), TIOA, TBP and TTA studies. The 228Th was measured by alpha spectroscopy. Two methods of alpha spectroscopy were employed. One method used thin samples made by evaporation. These samples were slowly evaporated on 
tantalum foils under an infrared lamp and flamed in a burner to red heat. The resulting thin samples were then counted with Si surface barrier detectors for alpha activity. The other method involved alpha scintillation spectroscopy. The sample solutions were added to $10 \mathrm{~mL}$ of Ecolite scintillation fluid and counted in an alpha scintillation counter. Corrections for quenching were obtained by counting known activities of 228 Th samples in different solutions.

\section{6. $238 \mathrm{Pu}$}

Plutonium, like thorium, is a member of the actinide series. The stable oxidation state of plutonium is $4+$, but other oxidation states can be prepared. Like thorium, plutoniurn is not a true rutherfordium homolog, since it is not a member of the Group 4 series. Tetravalent plutonium was used in the studies for the same reasons as thorium.

The $238 \mathrm{Pu}$ was prepared in a $6 \mathrm{M} \mathrm{HCl}$ solution. The $238 \mathrm{pu}$ stock solutions were evaporated and redissolved in a $12 \mathrm{HCl}$ solution. Enough $\mathrm{HCl}$ was added to give a specific activity of $360 \mathrm{nCi} / 30 \mu \mathrm{L}$. The $238 \mathrm{Pu}$ was measured by the same alpha spectroscopic methods as 228 Th and was used in the TIOA, TBP and TTA studies.

\section{Radiation Detection}

All samples were assayed for radioactivity to determine the distribution coefficient or percent extraction. The isotopes $95 \mathrm{Zr},{ }^{144} \mathrm{Ce},{ }^{152} \mathrm{Eu},{ }^{162} \mathrm{Hf}$, and $169 \mathrm{Hf}$ were assayed for gamma activity with either lithium drifted germanium or intrinsic germanium detector. A Canberra System 100 spectometer was used for data acquisition and analysis.

The alpha-emitting isotopes $228 \mathrm{Th}$ and $238 \mathrm{Pu}$ were assayed by two methods. Thin samples were assayed with surface barrier or passivated ion. implanted planar silicon (PIPS) detectors. An ORTEC ADCAM acquisition system was used for data acquisition and analysis. A second method for 
assaying alpha activity was scintillation. A Wallac 1219 Liquid Scintillation Counter was used with samples in Ecolite scintillation fluid. Data analysis was done with the accompanying DataMaster software. Detection of rutherfordium was done with a PIPS detector using our Realtime Acquisition Graphics System (RAGS) acquisition software [LAR87]. 


\section{Chapter 3. Chemical Properties of Rutherfordium}

\section{A. Introduction}

The chemical properties of elements can be studied in a variety of ways. Usual spectroscopic methods rely upon the interaction of the element or complex with photons (IR, UV-Vis, X-ray diffraction, etc.), a magnetic field (NMR), or solution (titration). However, for the study of transactinide elements the aforementioned methods do not have the sensitivity required for atom-at-atime chemistry. The transactinide elements can only be detected by their radioactive decay using current method's. This fact dictates the experimental methods used in transactinide research.

The chemistry of rutherfordium and it homologs was explored using organic ligands in liquid-liquid extraction systerns. The metal-ligand complex created is soluble in the organic phase. The aqueous phase was varied to study the effect on metal ligand complexation. Counting the radioactivity in each phase determined the amount of metal bound by the ligand. Insight into the chemistry of rutherfordium was achieved by comparing its behavior with those of its homologs.

\section{B. Catechol Ligands}

\section{Introduction}

Catechol based plutonium complexing agents have been developed in response to the biological hazards associated with plutonium contamination. Once ingested, only a small amount of plutonium is excreted from the body. The chelating agent diethylenetriaminepentaacetic acid (DTPA) is currently used for plutonium detoxification. Unfortunately, DTPA is a nonspecific ligand removing many soluble metals from body fluids [COH75]. Additionally, DTPA is unable to remove plutonium once it has left circulation or after hydrolysis of the 
metal has occurred [SMI72]. Synthesis of a plutonium specific ligand was undertaken to overcome these problems.

In vivo, plutonium has a tetravalent oxidation state. In mammals, $\mathrm{Pu} 4+$ and $\mathrm{Fe}^{3+}$ have similar transport properties. In the body, plutonium becomes bound to ferritin, an iron storage protein in liver cells. Eventually, plutonlum is incorporated into long-term iron storage sites [DUR75], [TAY72]. A reason for the common behavior of $\mathrm{Pu}^{4+}$ and $\mathrm{Fe}^{3+}$ is their comparable charge per ionic radius ratio (4.2 and 4.6 e/Å respectively). In addition, both $\mathrm{Pu}^{4+}$ and $\mathrm{Fe}^{3+}$ form highly insoluble hydroxides (Table 9) [RAY84]. However, tetravalent plutonium is usually eight coordinate, while trivalent iron is normally six coordinate.

\begin{tabular}{|ll|}
\hline & Table 9. $\mathrm{Pu}^{4+}$ and $\mathrm{Fe}^{3+}$ Hydroxide Data \\
$\mathrm{Fe}(\mathrm{OH})_{3} \leftrightarrow \mathrm{Fe}^{3+}+3 \mathrm{OH}^{-}$ & $\mathrm{K} \approx 10.38$ \\
$\mathrm{Fe}^{3+}+\mathrm{H}_{2} \mathrm{O} \leftrightarrow \mathrm{Fe}(\mathrm{OH})^{2+}+\mathrm{H}^{+}$ & $\mathrm{K}=0.0009$ \\
$\mathrm{Pu}(\mathrm{OH})_{4} \leftrightarrow \mathrm{Pu}^{4+}+4 \mathrm{OH}^{-}$ & $\mathrm{K}=10.55$ \\
$\mathrm{Pu} 4++\mathrm{H}_{2} \mathrm{O} \leftrightarrow \mathrm{Pu}(\mathrm{OH})^{3+}+\mathrm{H}^{+}$ & $\mathrm{K}=0.031$ (in $\mathrm{HClO}_{4}$ )
\end{tabular}

The similarities of $\mathrm{Pu}^{4+}$ and $\mathrm{Fe}^{3+}$ offer a biomimetic approach to development of a tetravalent plutonium sequestering agent. Siderophores, low molecular weight iron chelators produced by bacteria, can be used as models for a tetravalent plutonium sequestering agent. Siderophores usually contain hydroxamate or catecholate functional groups (Figure 5). The functional group complexation usually forms chelate rings which increase the stability compared to complex formation of lone oxygen anions [HUH72]. The catecholate functional group forms stronger complexes with tetravalent plutonium than the 
hydroxamate anion. This is due to the higher charge and stronger basicity of the catecholate anion. For this reason, catecholate was chosen as the functional group for synthetic multidentate chelating agents for the sequestering of tetravalent plutonium.

\section{Figure 5. Siderophore Functional Groups}<smiles>[R]c1cccc(O)c1O</smiles><smiles>[R]C(=O)N([R])O</smiles>

Catecholate Hydroxamate

The series of polydentate catechol ligands synthesized was originally used for tests on plutonium extraction from mice. The addition of electron withdrawing groups on the catechol improved the water solubility, stability to air and actinide affinity of the ligands [RAY84]. In addition, the withdrawing groups would make the ligands more acidic. The electron withdrawing substituents employed were sulfinate, nitrate and carboxylate. The synthesized ligands were tested for complexation with tetravalent cerium and trivalent europium. DTPA was tested with tetravalent thorium and trivalent europium. The tetravalent tracer experiments would indicate if the polydentate catechol ligands would be useful in extraction of rutherfordium. The charge per ionic radius ratio for the tracers is given in Table 10.

\section{Syntheses}

Intermediate products were monitored by melting point. UV-Vis spectra were taken on a Hewlett Packard 8450A Diode Array Spectrophotometer. Infrared spectra (KBr disks) were recorded on a Nicolet 5DX Infrared 
Spectrometer. ${ }^{1 H}$ NMR spectra were done on the UBC-200 spectrometer, and elemental analysis was performed by Analytical Services, Chemistry Department, Inniversity of California, Berkeley. Spermine was obtained from Ames Laboratories, and 2,3-dimethoxybenzoic acid was purchased from Aldrich Chemical Company.

Table 10. Ce, Eu, and Th Charge Per Ionic Radius

\begin{tabular}{llcl} 
Element & $\begin{array}{l}\text { Coordination } \\
\text { Number }\end{array}$ & ionic Radius (A) & $\mathbf{e} / \AA$ \\
$\mathrm{Ce}$ & 6 & 0.87 & 4.6 \\
$\mathrm{Ce}$ & 8 & 0.97 & 4.1 \\
$\mathrm{Eu}$ & 6 & 0.95 & 3.2 \\
$\mathrm{Eu}$ & 8 & 1.07 & 2.8 \\
$\mathrm{Th}$ & 6 & 0.94 & 4.3 \\
$\mathrm{Th}$ & 8 & 1.05 & 3.8 \\
\hline
\end{tabular}

Reference [SHA76]

a. 2,3-Dimethoxybenzoyl chloride (1)

In a $250 \mathrm{~mL}$ round-bottom flask, 2,3-dimethoxybenzoic acid (10.2 g, 56.0 $\mathrm{mmol})$ and $\mathrm{SOCl}_{2}(50.0 \mathrm{~mL})$ were heated at reflux under argon for 4 hours (Figure 6). The $\mathrm{SOCl}_{2}$ was removed by vacuum distillation. This procedure is shown in Figure 6.

Figure 6. 2,3-Dimethoxybenzoyl Chloride Synthesis<smiles>COc1cccc(C(=O)O)c1OC</smiles> 


\section{b. $\quad N, N^{\prime}, N^{\prime \prime}, N^{\prime \prime}-$ Tetrakis (2,3-dihydroxybenzoyl)-}

\section{1,5,10,14-tetraazatetradecane (3,4,3-LICAM) (2)}

The procedure for the synthesis of 3,4,3-LICAM is from Weitl et al. [WEI80] and shown in Figure 7. In a stoppered flask in a $60^{\circ} \mathrm{C}$ oil bath spermine $(2.0 \mathrm{~g}$, $9.9 \mathrm{mmol}), 1\left(8.02 \mathrm{~g}, 40.0 \mathrm{mmol}^{2}, \mathrm{NEt}_{3}(5.87 \mathrm{~mL})\right.$ and $\mathrm{N}, \mathrm{N}$-dimethylacetamide (DMAA) $(40 \mathrm{~mL})$ were stirred in a $60^{\circ} \mathrm{C}$ oil bath for 20 hours. The reaction mixture was evaporated, leaving an oily residue which was partitioned between $\mathrm{H}_{2} \mathrm{O}$ and $\mathrm{CH}_{2} \mathrm{Cl}_{2}$. The $\mathrm{CH}_{2} \mathrm{Cl}_{2}$ layer was washed with $0.1 \mathrm{M} \mathrm{HCl}, \mathrm{H}_{2} \mathrm{O}$, then 0.1 $\mathrm{M} \mathrm{NaOH}$, before drying with $\mathrm{MgSO}_{4}$.

The $\mathrm{CH}_{2} \mathrm{Ol}_{2}$ layer was eluted from an alumina column $(101.5 \mathrm{~mL}$ resin bed, column diameter $=2.2 \mathrm{~cm}$ ) using mixtures of $0.5 \% \mathrm{MeOH}$ in $\mathrm{CH}_{2} \mathrm{Cl}_{2}$. Elutions were done using $10 \mathrm{C} \mathrm{mL}$ of each $\mathrm{MeOH}-\mathrm{CH}_{2} \mathrm{Cl}_{2}$ solution. The solutions were $0 \%, 1 \%, 2 \%, 3 \%, 4 \%$ and $5 \% \mathrm{MeOH}$. The product N, N', N', N'"-Tetrakis $(2,3$ dimethoxy-benzoyl)-1,5,10,14-tetraazatetradecane (3) (methyl protected LICAM) eluted in $2-4 \% \mathrm{MeOH}$ solution as shown by TLC $\left(5 \% \mathrm{MeOH}-\mathrm{CH}_{2} \mathrm{Cl}_{2}\right.$ solvent). The eluant was evaporated, leaving 3 as a glassy solid.

The glassy solid product was dissolved in $\mathrm{CH}_{2} \mathrm{Cl}_{2}(16 \mathrm{~mL})$. For methyl deprotection, the resulting solution was added dropwise to $2 \mathrm{~mL}$ of concentrated $\mathrm{BBr}_{3}$ stirring under argon in a $50 \mathrm{~mL}$ round-bottom flask in a room temperature bath. A white precipitate immediately formed and was allowed to stir for 10 hours.

The white precipitate was hydrolyzed with $50 \mathrm{~mL}$ of $\mathrm{H}_{2} \mathrm{O}$, filtered and $\mathrm{H}_{2} \mathrm{O}$ washed. The white product which resulted from the hydrolysis was then vacuum dried at room temperature over $\mathrm{P}_{2} \mathrm{O}_{5} / \mathrm{NaOH}$. This yielded 3,4,3-LICAM (2) $(5.25 \mathrm{~g}, 70.7 \%): \operatorname{IR}(\mathrm{Nujol}) 3700-2500 \mathrm{~cm}^{-1} \mathrm{CH}, \mathrm{OH}, 1640-1590 \mathrm{~cm}^{-1} \mathrm{CON}$;

$1 \mathrm{H} \mathrm{NMR}\left(2 \mathrm{OMMH}, \mathrm{D}_{2} \mathrm{O}\right)$ 21.5-2.5 $\mathrm{NCH}_{2} \mathrm{CH}_{2}$ (broad 8H), 0 3.3-4.3 $\mathrm{NCH}_{2}$ (broad $12 \mathrm{H}), 27.0-8.0 \mathrm{ArH}($ broad $\mathrm{m} \mathrm{8H})$ 
Anal. Calculated (Found) for $\mathrm{C}_{38} \mathrm{H}_{42} \mathrm{~N}_{4} \mathrm{O}_{12} \cdot 1 \mathrm{H}_{2} \mathrm{O}:$ C, $59.68 \%(60.62 \%), \mathrm{H}$, $5.80 \%(6.25 \%), N, 7.33 \%(6.71 \%)$.

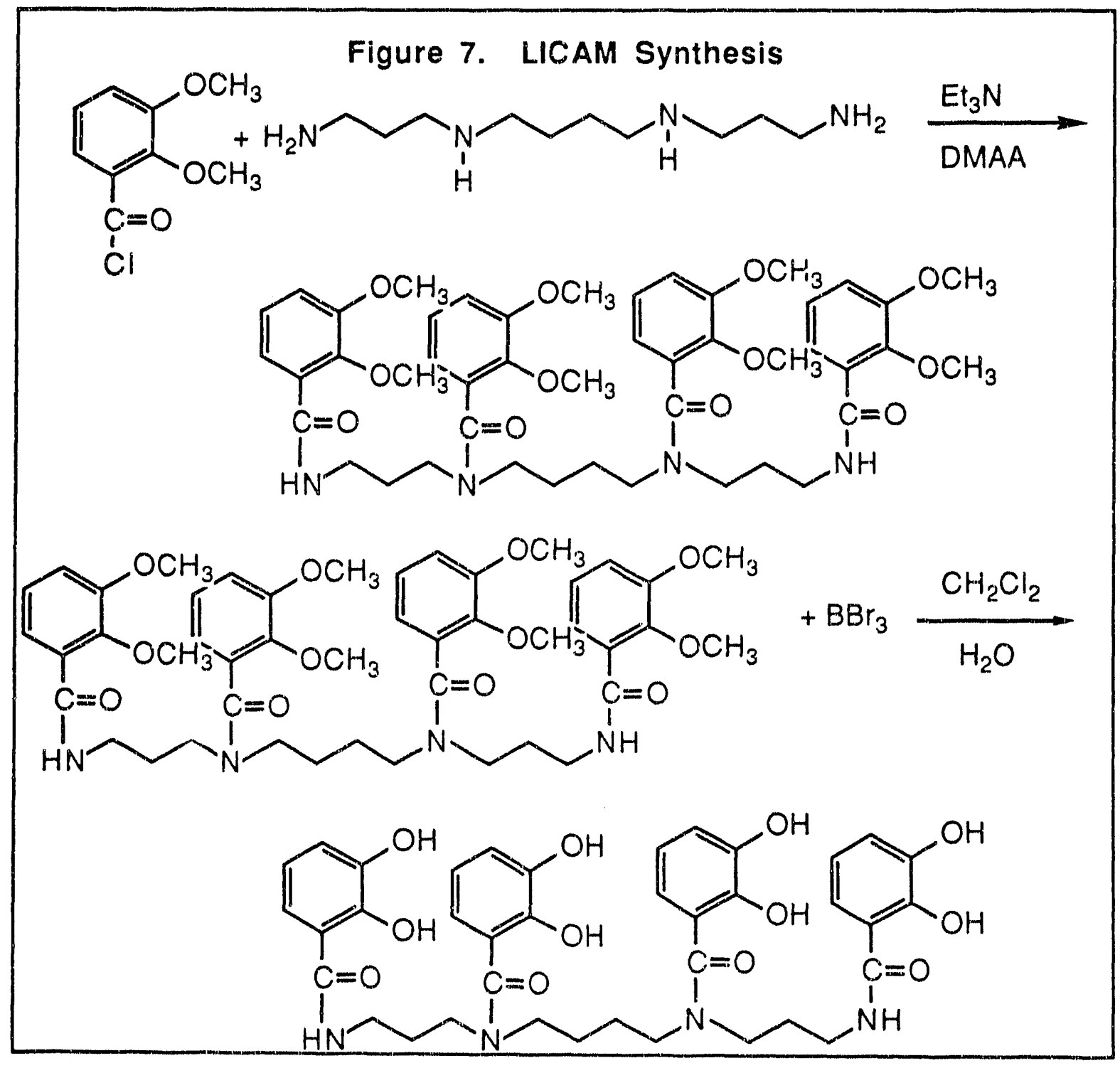

c. $\quad N, N^{\prime}, N^{\prime \prime}, N^{\prime \prime \prime}$-Tetrakis(2,3-dihydroxy-5-sulfobenzoyl)-1,5,10,14-tetraazatetradecane (3,4,3-LICAMS)(4)

The procedure for the synthesis of 3,4,3-LICAMS is from Weitl et al. [WEI80] and is shown in Figure 8 . Dry $2(5.1 \mathrm{~g}, 6.83 \mathrm{mmol})$ dissolved in $15 \%$ fuming $\mathrm{H}_{2} \mathrm{SO}_{4}(30 \mathrm{~mL})$ was stirred in a room temperature bath under argon for 10 
hours. The reaction mixture was poured over ice and adjusted to $\mathrm{pH}=4$ with $10.0 \mathrm{M} \mathrm{NaOH}$. Two equivalent volumes of $\mathrm{MeOH}$ were added, forming a white, salt precipitate $\left(\mathrm{Na}_{2} \mathrm{SO}_{4}\right)$. The salt was filtered off and washed well with 1:1 $\mathrm{MeOH}: \mathrm{H}_{2} \mathrm{O}$.

The filtrate and wash were evaporated to dryness and redissolved in a minimum amount of $\mathrm{H}_{2} \mathrm{O}$. One equivalent of $\mathrm{MeOH}$ and $\mathrm{EtOH}$ was added. An inorganic salt precipitate formed, which was filtered off. To the filtrate, 3 equivalents of $\mathrm{Et}_{2} \mathrm{O}$ were added. A white precipitate formed, which was filtered off and dried at room temperature over $\mathrm{P}_{2} \mathrm{O}_{5} / \mathrm{NaOH}$. This precipitate is 3,4,3LICAMS(4) (5.53g, 70.1\%): IR (Nujol) $3381-2500 \mathrm{~cm}^{-1} \mathrm{CH}, \mathrm{OH}, 1640-1590$ $\mathrm{cm}^{-1}$ CON, $1470 \mathrm{~cm}^{-1}, 1420 \mathrm{~cm}^{-1}, 1380 \mathrm{~cm}^{-1}, 1210-1180 \mathrm{~cm}^{-1}, 1100 \mathrm{~cm}^{-1}$ $\mathrm{SO}_{3} \mathrm{Na} ;{ }^{1} \mathrm{H} \mathrm{NMR}\left(200 \mathrm{MHz}, \mathrm{D}_{2} \mathrm{O}\right)$ 21.3-2.0 $\mathrm{NCH}_{2} \mathrm{CH}_{2}$ (broad 8H), ว 2.8-3.9 $\mathrm{NCH}_{2}$ (broad 12H), 2 7.0-7.8 ArH (broad $\mathrm{m} \mathrm{8H}$ ).

Anal Calculated (Found). for $\mathrm{C}_{38} \mathrm{H}_{38} \mathrm{~N}_{4} \mathrm{O}_{24} \mathrm{~S}_{4} \mathrm{Na}_{4} \cdot 6 \mathrm{H}_{2} \mathrm{O}$ : C, $36.12 \%$ (36.28), H, $3.99 \%(4.03 \%), N, 4.44 \%$ (3.98\%), S, $10.15 \%$ (9.93\%).

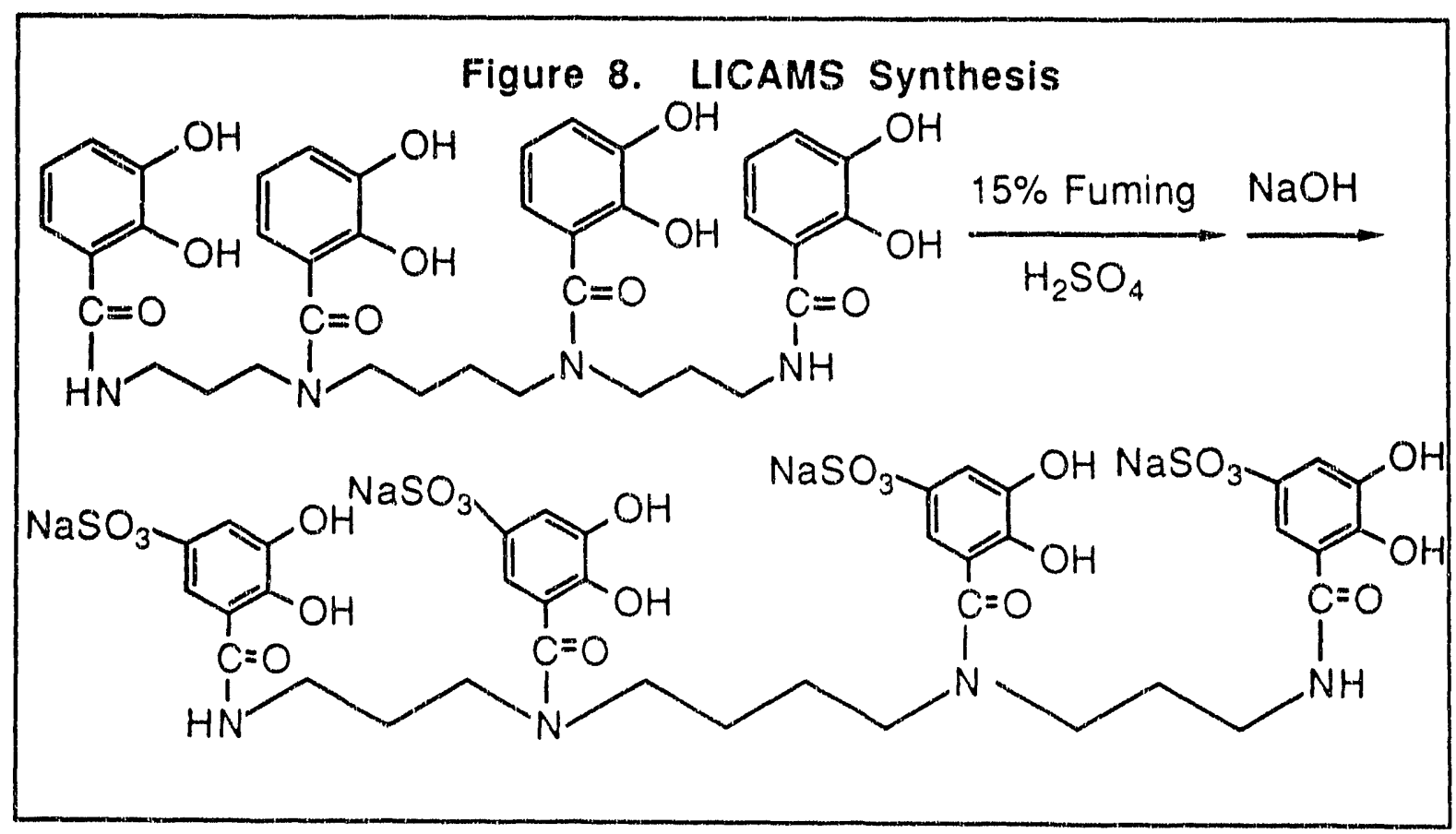




\section{d. $\quad \mathrm{N}, \mathrm{N}^{\prime}, \mathrm{N}^{\prime \prime}, \mathrm{N}^{\prime \prime}$-'Tetrakis(2,3-dihydroxy-5-nitro-}

\section{benzoyI)-1,5,10,14-tetraazatetradecane (3,4,3-LICAMN)}

Synthesis for 4-nitro-2,3-dimethoxybenzoic acid (6) is from an unpublished source [WE179] and is presented in Figure 9. To a $100 \mathrm{~mL}$ round-bottom flask, 2,3-dimethoxybenzoic acid $(10.0 \mathrm{~g}, 54.9 \mathrm{mmol})$ and glacial acetic acid $(40 \mathrm{~mL})$ were added to concentrated $\mathrm{HNO}_{3}(5.5 \mathrm{~mL})$. The resulting solution was heated at $95^{\circ} \mathrm{C}$ under reflux for 1 hour. Addition of two volumes of cold $\mathrm{H}_{2} \mathrm{O}$ to the reaction mixture formed a white precipitate which was filtered off, $\mathrm{H}_{2} \mathrm{O}$ washed, and oven dried. Purification of this white product was done by recrystallization from acetic acid. TLC with 1:1 Methanol:10\% $\mathrm{CH}_{3} \mathrm{COONH}_{4}$ showed one spot at $R_{f}=0.88$.

The acid chloride of (6) was made by heating at reflux with $\mathrm{SOCl}_{2}$ for 6 hours. Vacuum distillation left a yellow solid. This acid chloride was used to make 3,4,3-LICAMN by following the synthetic procedure for 2 . The product ligand 3,4,3-LICAMN (4.87 g, 79.9\%) was produced: IR (Nujol) $3700-2500 \mathrm{~cm}^{-1}$ $\mathrm{CH}, \mathrm{OH}, 1640-1590 \mathrm{~cm}^{-1} \mathrm{CON} ;{ }_{1}^{1} \mathrm{H} \mathrm{NMR}\left(200 \mathrm{MHz}, \mathrm{D}_{2} \mathrm{O}\right)$ 21.5-2.5 $\mathrm{NCH}_{2} \mathrm{CH}_{2}$ (broad 8H), ว 3.3-4.3 $\mathrm{NCH}_{2}$ (broad 12H), 2 7.0-8.0 ArH (broad $\mathrm{m} 8 \mathrm{H}$ )

Anal. Calculated (Found) for $\mathrm{C}_{38} \mathrm{H}_{42} \mathrm{~N}_{4} \mathrm{O}_{12} \cdot 1 \mathrm{H}_{2} \mathrm{O}: \mathrm{C}, 59.68 \%(60.62 \%), \mathrm{H}$, $5.80 \%(6.25 \%), N, 7.33 \%(6.71 \%)$.

\section{e. $\quad N, N^{\prime}, N^{\prime \prime}, N^{\prime \prime \prime}$-Tetrakis(2,3-dihydroxy-4-carboxy"}

\section{benzoyl)-1,5,10,14-tetraazatetradecane (3,4,3-LICAMC) (7)}

The procedure for this synthesis is from Weitl et al. [WE.181] and is shown in Figure 10. Dimethyl 2,3-dihydroxyterephthalate (8), was used as the starting material. $8(3.60 \mathrm{~g}, 15.9 \mathrm{mmol})$ and $\mathrm{K}_{2} \mathrm{CO}_{3}(4.40 \mathrm{~g}, 31.8 \mathrm{mmol})$ were placed in a $250 \mathrm{~mL}$ round-bottom flask with dropwise addition of acetone $(150 \mathrm{~mL})$ and $\left(\mathrm{CH}_{3}\right)_{2} \mathrm{SO}_{4}(3.02 \mathrm{~mL}, 31.8 \mathrm{mmol})$. The reaction mixture was heated at $80^{\circ} \mathrm{C}$ under reflux for 24 hours. At the end of the refiux, salts were filtered off while the 
solution was still hot. The solution was evaporated after filtration, yielding the product dimethyl 2,3-dimethoxy-terephthalate (9).

Figure 9. LICAMN Synthesis<smiles>COc1cccc(C(=O)O)c1OC</smiles><smiles>COc1cc([N+](=O)[O-])cc(C(=O)O)c1OC</smiles>

$\mathrm{O}_{2} \mathrm{~N}$<smiles>COc1cccc(C(=O)O)c1OC</smiles>
reflux<smiles>COc1cc([N+](=O)[O-])cc(C(=O)Cl)c1OC</smiles><smiles>COc1cc([N+](=O)[O-])cc(C(=O)Cl)c1OC</smiles><smiles>COc1ccc([N+](=O)[O-])cc1OC</smiles>
HN $\mathrm{C}=\mathrm{O}$ i<smiles>C1CCCCC1</smiles><smiles>C1CCCC1</smiles><smiles>CCCCN(CC)C(=O)CC</smiles><smiles>CCCCCN(C=O)CC</smiles>
$\mathrm{C}^{\mathrm{C}=\mathrm{O}}+\mathrm{BBr}_{3} \rightarrow$<smiles>O=Cc1cc([N+](=O)[O-])cc(O)c1O</smiles><smiles>O=Cc1cc([N+](=O)[O-])cc(O)c1O</smiles><smiles>CC(=O)c1cc([N+](=O)[O-])cc(O)c1O</smiles>
$\mathrm{O}_{2} \mathrm{~N}$<smiles>Cc1ccccc1</smiles>
$H{ }^{\prime}$ 
To 9 (2.22 g, $8.73 \mathrm{mmol}$ ) dissolved in $\mathrm{CH}_{3} \mathrm{OH}(120 \mathrm{~mL}), \mathrm{NaOH}(0.35 \mathrm{~g}, 8.75$ mmol) in $\mathrm{H}_{2} \mathrm{O}(5 \mathrm{~mL})$ was added. The solution was heated at reflux for 8 hours. After reflux, $75 \%$ of its volume was removed by evaporation. Addition of two equivalent volumes of acetone formed a salt which was filtered off and washed well with acetone. Addition of two volumes of $\mathrm{Et}_{2} \mathrm{O}$ to the filtrate and wash formed a white precipitate, sodium dimethyl 2,3-dimethoxyterephthalate (10).

Next, the chloride of 10 was made. To $10(1.86 \mathrm{~g}, 7.15 \mathrm{mmol})$ in a roundbottom flask, $\mathrm{SOCl}_{2}(20 \mathrm{~mL})$ was added and stirred for 12 hours under a Drierite tube. One volume of $\mathrm{CCl}_{4}$ was added to the solution, followed by filtration to remove $\mathrm{NaCl}$. The solution was then vacuum distilled, washed and redistilled with $\mathrm{CCl}_{4}$ three times. This yielded methyl 2,3-dimethoxy-terephthaloyl chloride (11).

For the final reaction step, THF $(50 \mathrm{~mL})$ was added to $11(1.6 \mathrm{~g}, 6.49 \mathrm{mmol})$, spermine $(0.33 \mathrm{~g}, 1.62 \mathrm{mmol})$ and $\mathrm{Et}_{3} \mathrm{~N}(1 \mathrm{~mL})$. A white precipitate formed immediately. The reaction mixture was stirred for 17 hours at room temperature in a stoppered flask. The solution was filtered and washed with THF, removing the $\mathrm{NEt}_{3} \cdot \mathrm{HCl}$ precipitate. Evaporation of the filtrate solution gave a light brown oil. The oil was redissolved in $\mathrm{CH}_{2} \mathrm{Cl}_{2}$ and eluted through a silica column using a $0.5 \% \mathrm{MeOH}$ gradient in $\mathrm{CH}_{2} \mathrm{Cl}_{2}$. The product N,N',N",N'"-Tetrakis (2,3dihydroxy-4-carbomethoxy-benzoyl)-1,5,10,14-tetraazatetradecane (methyl protected 3,4,3-LICAMC) (12) eluted in $2-4 \% \mathrm{MeOH}$.

Deprotection of $\mathbf{1 2}$ was done using a slightly modified version for the deprotection of 3,4,3-LICAM. After hydrolysis with $\mathrm{H}_{2} \mathrm{O}$, the resulting solution was mixed for 5 hours. A tan precipitate formed, which was removed by filtration, $\mathrm{H}_{2} \mathrm{O}$ washed, and slurried in $100 \mathrm{~m} !$ of $\mathrm{H}_{2} \mathrm{O}$. The $\mathrm{pH}$ of the slurry was adjusted to 7 using $0.1 \mathrm{M} \mathrm{NaOH}$. The addition of $0.1 \mathrm{M} \mathrm{HCl}$ gave a precipitate, 3,4,3-LICAMC $[0.93 \mathrm{~g},(68 \%)]$, which was removed by filtration, $\mathrm{H}_{2} \mathrm{O}$ washed and 
dried over $\mathrm{P}_{2} \mathrm{O}_{5} / \mathrm{NaOH}:$ IR (Nujol) $3360-2500 \mathrm{~cm}^{-1} \mathrm{CH}, \mathrm{OH}, 1679 \mathrm{~cm}^{-1} \mathrm{COOH}$, $1609 \mathrm{~cm}^{-1} \mathrm{CON}, 1321 \mathrm{~cm}^{-1}, 1229 \mathrm{~cm}^{-1}, 1180 \mathrm{~cm}^{-1} \mathrm{CN}$ CO double bond; ${ }^{1} \mathrm{H}$

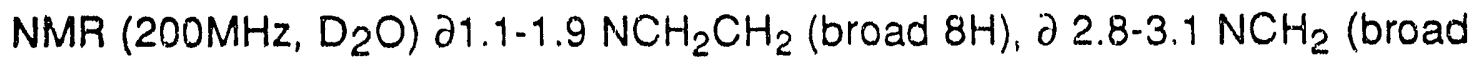
12H), 2 7.2-7.8 ArH (broad $\mathrm{m} 8 \mathrm{H}$ )

Anal. Calculated (Found) for $\mathrm{C}_{42} \mathrm{H}_{42} \mathrm{~N}_{4} \mathrm{O}_{20} \cdot 4 \mathrm{H}_{2} \mathrm{O}: \mathrm{C}, 50.70 \%(50.10 \%), \mathrm{H}$, $5.07 \%(4.83 \%), N, 5.63 \%(5.43 \%)$.

Figure 10. LICAMC Synthesis

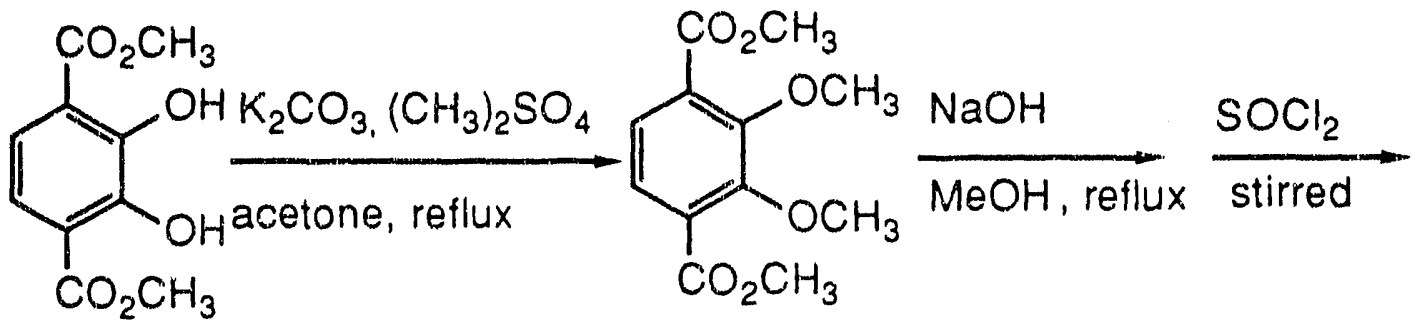<smiles>CCN(CC)CCCC(=O)c1ccc(C(=O)Cl)c(OC)c1OC</smiles><smiles>O=C(O)c1ccc(C(=O)NCCCN(CCCCN(CCCNC(=O)c2ccc(C(=O)O)c(O)c2O)C(=O)c2ccc(C(=O)O)c(O)c2O)C(=O)c2ccc(C(=O)O)c(O)c2O)c(O)c1O</smiles>

\section{Tracer Extractions}

The LICAM, LICAMS, LICAMN, and LICAMC ligands were tested using ion exchange methods. Catechol and DTPA were examined in addition to the 
LICAM series of ligands. The catechol and DTPA were purchased from Aldrich and used as received. The system for investigation of the ligand-metal complex involved the mixing of $40 \mu \mathrm{L}$ of a buffer, $30 \mu \mathrm{L}$ of $0.1 \mathrm{M}$ ligand solution, and 30 $\mu \mathrm{L}$ tracer in $0.2 \mathrm{M} \mathrm{HCl}$. The resulting solution was pre-equilibrated by mixing for 2 minutes. The pH was measured, and the solution was added to a $300 \mu \mathrm{L}$ resin bed of AG-MP50 cation exchange resin in a $0.6 \mathrm{~cm} \times 7 \mathrm{~cm}$ column and eluted with $1 \mathrm{~mL}$ of $\mathrm{H}_{2} \mathrm{O}$. The eluant was collected and counted for activity. Complexed tracer elutes through the cation exchange column, while uncomplexed tracer is absorbed by the resin. Comparison of the known activity of the tracer to the eluted activity gave the percentage of metal complexed to the ligand as a function of $\mathrm{pH}$.

The buffer systems investigated were sodium acetate buffers, sodium acetate- $\mathrm{NaOH}$ buffers, sodium acetate-carbonate buffers, and ammonium acetate- $\mathrm{NaOH}$ buffers. The sodium acetate buffers were made by mixing $1 \mathrm{M}$ $\mathrm{CH}_{3} \mathrm{COOH}$ and $1 \mathrm{M} \mathrm{CH}_{3} \mathrm{COONa}$. Solutions of $1 \mathrm{M} \mathrm{CH}_{3} \mathrm{COOH}$ and $1 \mathrm{M} \mathrm{CO}_{3} \mathrm{Na}_{2}$ were used for the acetate-carbonate buffers. Mixing solutions of $1 \mathrm{M}$ $\mathrm{CH}_{3} \mathrm{COONa}$ and $1 \mathrm{M} \mathrm{NaOH}$ were used to make the acetate- $\mathrm{NaOH}$ buffers. The ammonium acetate- $\mathrm{NaOH}$ buffers were made by mixing a $2 \mathrm{M} \mathrm{NH}_{4} \mathrm{OOCCH}$ : $2 \mathrm{M} \mathrm{NaOH}$ stock solution with $1 \mathrm{M} \mathrm{CH}_{3} \mathrm{COOH}$. The solutions were mixed to the desired buffer $\mathrm{pH}$. The carbonate buffer solutions were not used since tracer sorbed on the cation exchange resin is removed by carbonate. The $\mathrm{pH}$ range of the buffer solutions is given in Table 11.

An experiment was done to determine the amount of buffer necessary to adjust the $\mathrm{pH}$ of an experimental ligand-tracer system. The experimental tracer stock solutions are $0.2 \mathrm{M} \mathrm{HCl}$. The ligand solution was simulated using $\mathrm{H}_{2} \mathrm{O}$. For this experiment, $30 \mu \mathrm{L} \mathrm{H} \mathrm{H}_{2} \mathrm{O}$ and $30 \mu \mathrm{L} 0.2 \mathrm{M} \mathrm{HCl}$ were mixed. To this mixture, buffer was added $10 \mu \mathrm{L}$ at a time. The effect on $\mathrm{pH}$ as a function of 
buffer volume was measured. It was found that $40 \mu \mathrm{L}$ of buffer was needed to stabilize the $\mathrm{pH}$ of the solution. Table 12 shows the buffers and tracers used for each ligand investigated.

\section{Table 11. Buffer pH Range}

Buffer

sodium acetate

sodium acetate- $\mathrm{NaOH}$

sodium acetate-carbonate

ammonium acetate- $\mathrm{NaOH}$

\section{$\mathrm{pH}$ range}

\section{5-8.6}

9.0-14.0

$5.5-10.6$

$9.0-11.5$

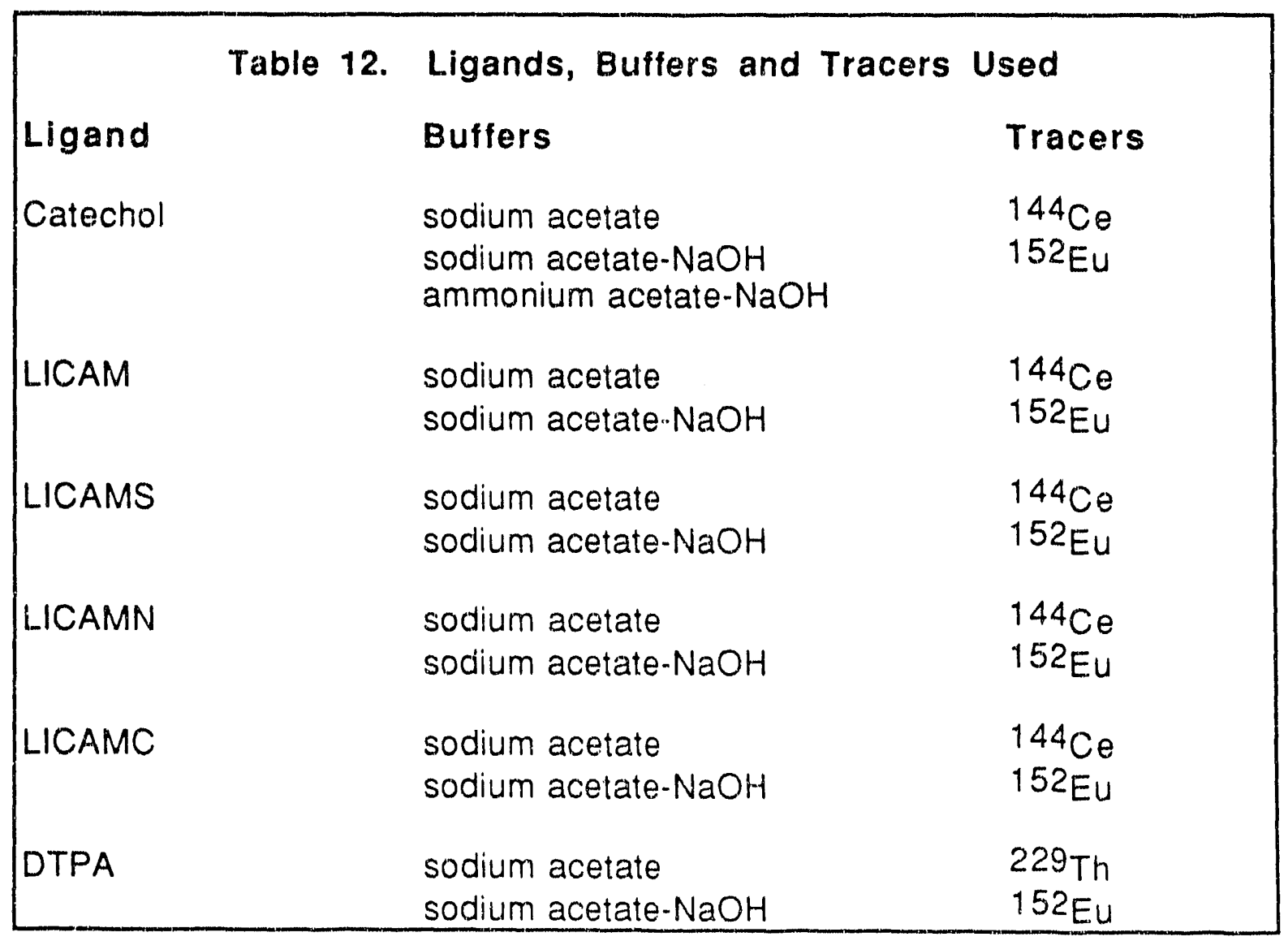




\section{Results}

The catechol complexation results, shown in Figure 11, indicate the ability of catechol to form metal-ligand species with both tetravalent cerium and trivalent europium in a similar $\mathrm{pH}$ range. Over the $\mathrm{pH}$ range examined, the complexation of tetravalent cerium and trivalent europium by catechol is similar. This result may indicate the ability of the LICAM ligand series to extract trivalent europium. The LICAM ligand series results are presented in Figures 12-15.

The results show the LICAM ligand series preferentially forms complexes with tetravalent cerium at high $\mathrm{pH}$. However, the ligands also extract europium. The ligand LICAM performed the best, giving approximately $100 \%$ cerium extraction at $\mathrm{pH}=10.5$. From there, the cerium extraction decreased as the $\mathrm{pH}$ increased. Europium behaves similarly, having a maximum extraction of $45 \%$ at $\mathrm{pH} 10.5$ and decreasing as the $\mathrm{pH}$ increases. Unfortunately, these initial tests indicate that the LICAM ligand extracts more trivalent actinide than acceptable for the experimental requirements. The alpha decay of the extracted trivalent actinides would interfere with the alpha decay detection of 261 Rf. The trivalent actinide extraction needs to be less than $10 \%$ for acceptable rutherfordium alpha spectroscopy.

The LICAMS ligand has similar complexation of tetravalent cerium and trivalent europium over the examined $\mathrm{pH}$ range. The cerium complexation is slightly greater at $\mathrm{pH}=10$. The europium complexation is prevalent at $\mathrm{pH}=13$. The LICAMN ligand shows a steady increase in cerium complexation in the 10 to $13 \mathrm{pH}$ range. In the same $\mathrm{pH}$ range, the europium complexation decreases to a minimum of $55 \%$ at $\mathrm{pH}=13$. The L.ICAMC ligand had a constant cerium complexation near $100 \%$ in the 8 to $13 \mathrm{pH}$ range. The europium extraction increased and reached a maximum of $85 \%$ complexation at about $\mathrm{pH}=8$. The 
europium complexation remained constant at $85 \%$ complexation in the 8 to 13 $\mathrm{pH}$ range.

The LICAM ligand series complexation of europium is not unexpected and has been reported [RAY84]. Complexation of europium with the LICAM ligand series occurs with partially deprotonated ligand. At high $\mathrm{pH}$, hydroxide also appears to be coordinated to the europium ligand complex [RAY84]. In fact, the same paper shows trivalent americium forms complexes of undetermined stability and stochiometry with LICAMS and LICAMC.

Figure 16 shows DTPA complexation with europium and thorium. The thorium complexation with DTPA reaches a maximum of $90 \%$ at $\mathrm{pH}=5$ and remains constant in the $\mathrm{pH}$ range of 5 to 10 . $\mathrm{By} \mathrm{pH}=12$, the thorium complexation decreased to $80 \%$. Europium complexation with DTPA reaches a maximum of about $100 \%$ at $\mathrm{pH}$ of 7 . The europium complexation then decreases with increasing $\mathrm{pH}$. A minimum extraction of $10 \%$ is reached at a $\mathrm{pH}$ of 13. It may be possible to use DTPA to separate rutherfordium from the trivalent actinides. However, the high $\mathrm{pH}$ necessary for the low europium extraction adds difficulties to an attempted experiment. Rutherfordium hydroxides may form, hindering formation of a Rf-DTPA complex and increasing the time need for complex formation. The additional time would be unacceptable when using 65-second ${ }^{261} \mathrm{Rf}$. 


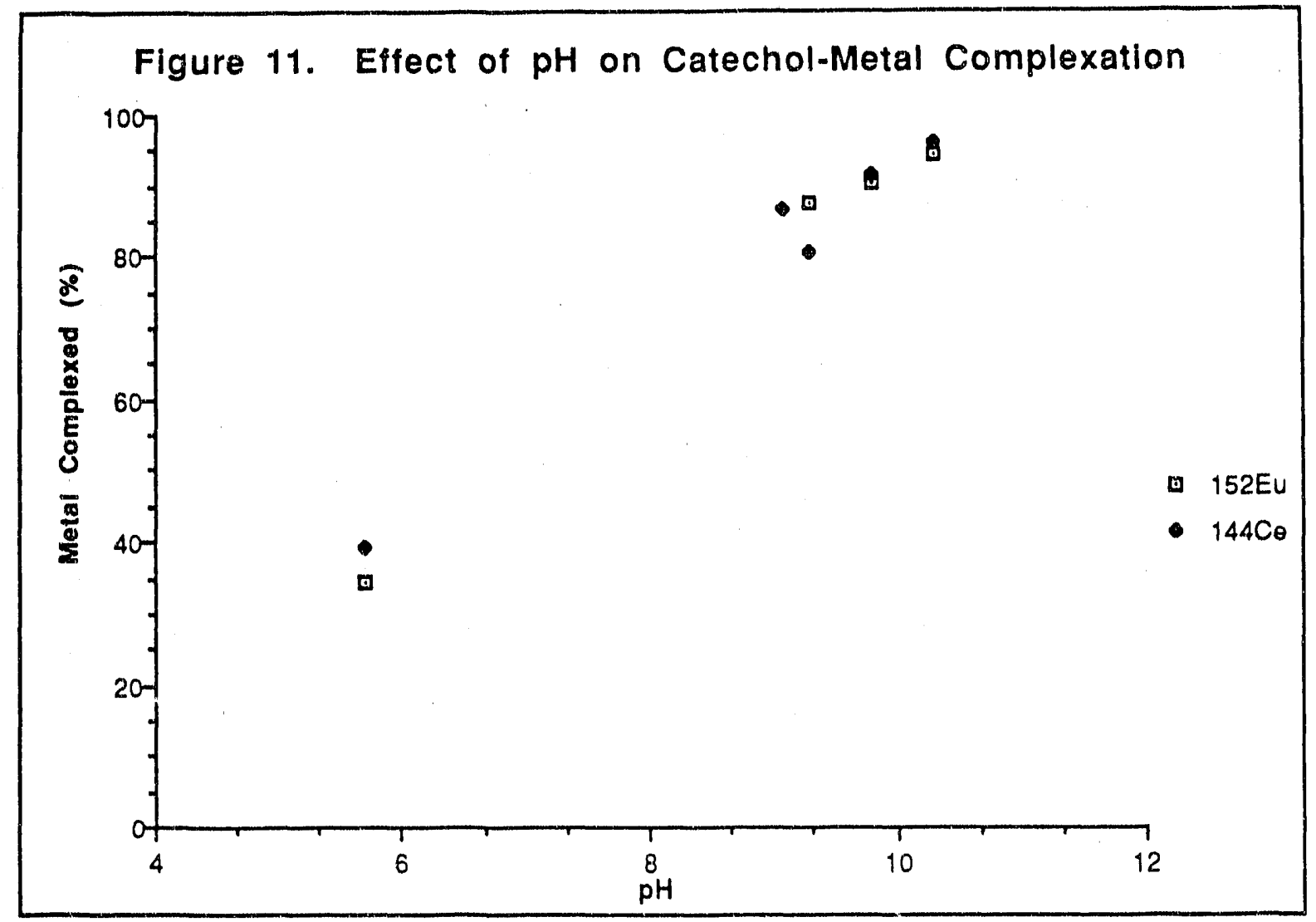

Figure 12. Effect of pH on LICAM-Metal Complexation

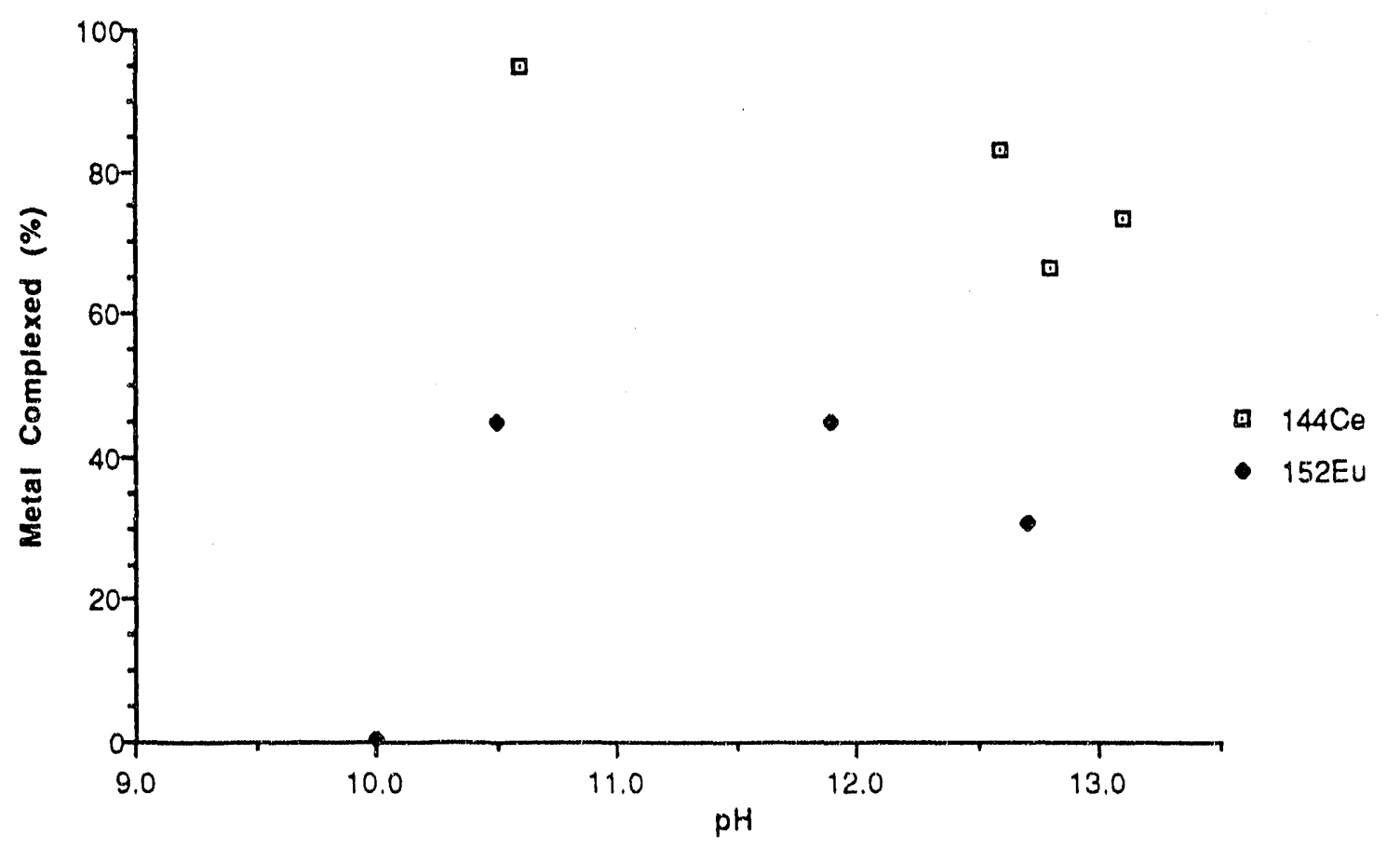



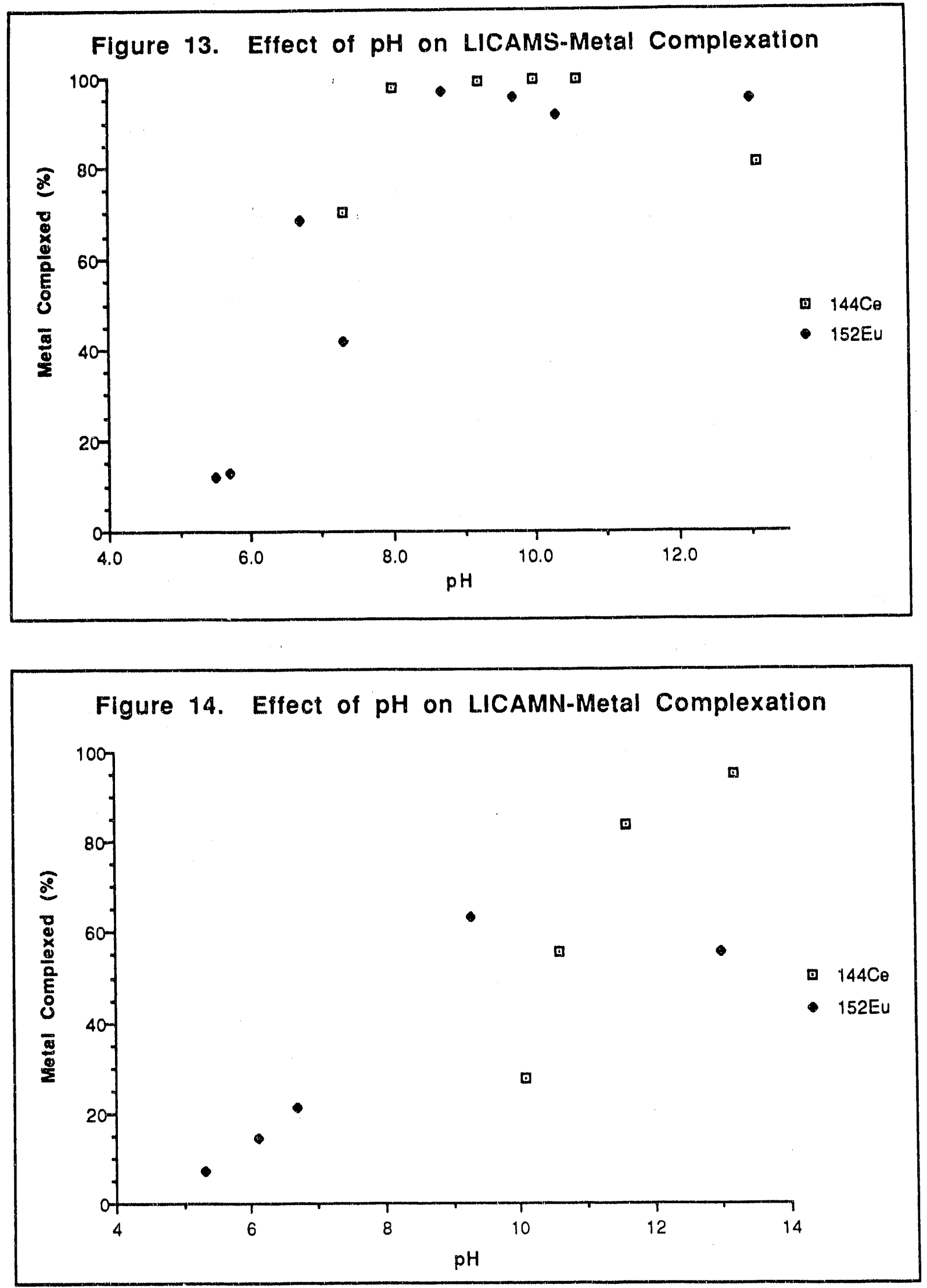


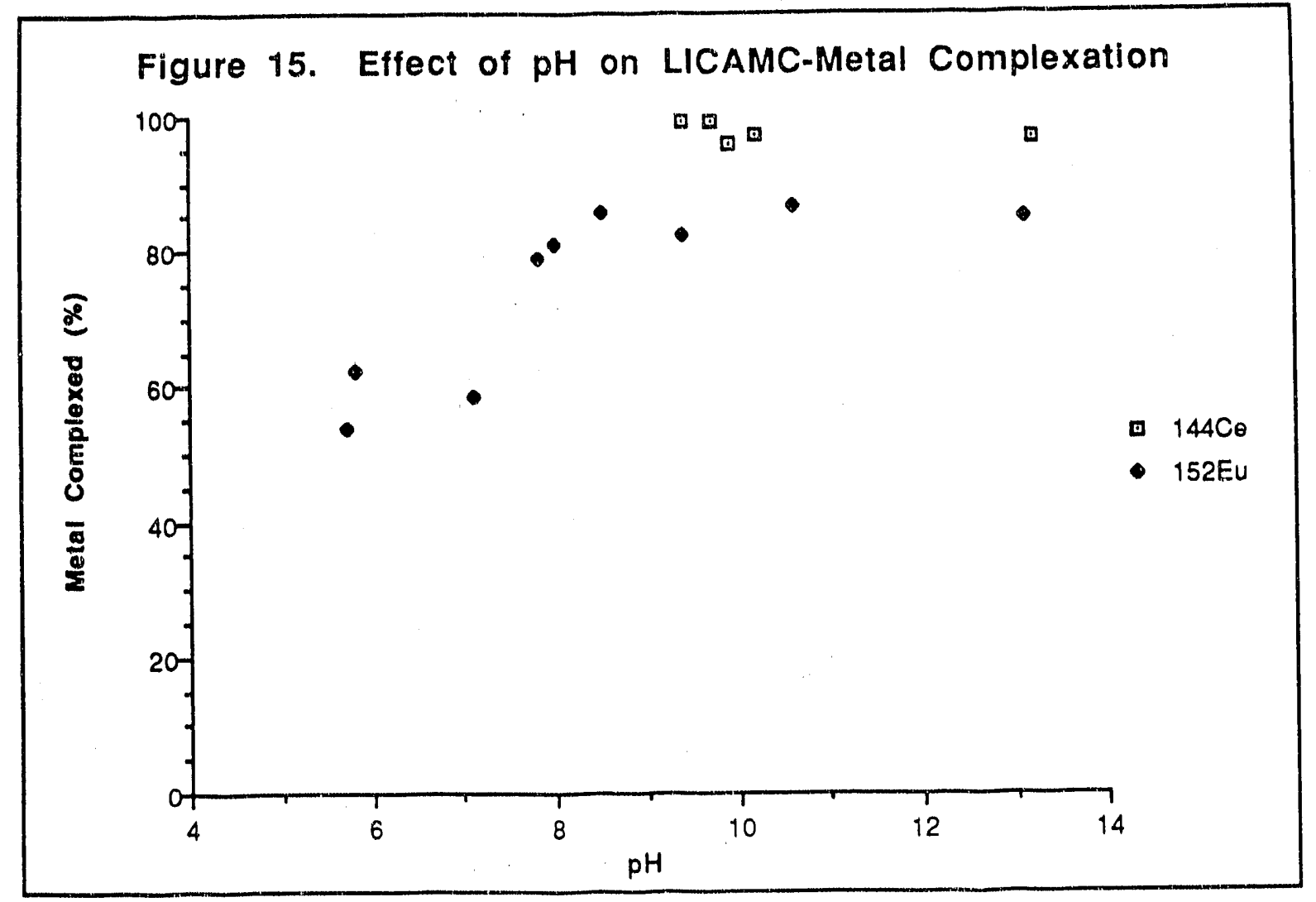

Figure 16. Effect of $\mathrm{pH}$ on DTPA-Metal Complexation

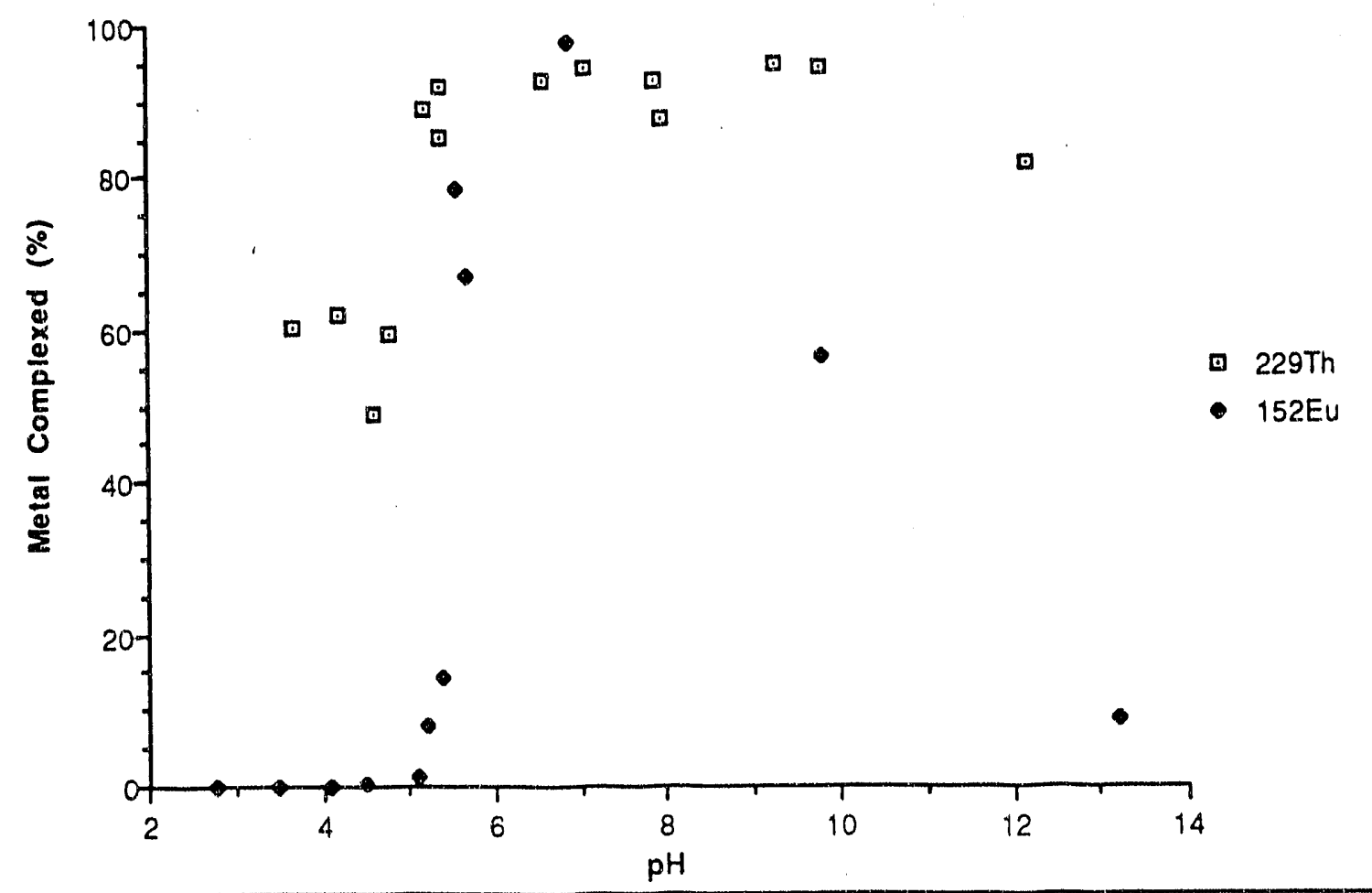




\section{Trialkylamine Ligand}

\section{Introduction}

The extraction systems discussed in this Section involve the use of high molecular weight amines. The extraction chemistry of these compounds is similar to the absorption of metal complexes on anion exchange resin where the formation of extractable anionic metal species controls the metal extraction. Additional parameters that affect the extraction of metal ions in the systems studied include aqueous ligand concentration, concentration of competitive ligands, diluent used, hydrogen ion concentration, competition from other anions which may complex to the cationic trialkylamine and the trialkylamine ligand itself.

To constitute good extractants, the amines must meet some fundamental requirements. Useful amine ligands for solvent extraction will be compatible with the organic diluent, have a large extraction power, low solubility in the aqueous phase, be stable in the presence of concentrated acid, and rapidly separate from the aqueous phase. Saturated nitrogen compounds successfully fill these requirements [MAR69]. For this reason, tertiary amines were studied.

The diluent used has been shown to affect the behavior of the extraction systems. Enormous changes in the distribution coefficient $\left(K_{d}=\right.$ (cts organic/volume organic)/(cts aqueous/volume aqueous)) have been produced with changes in the organic diluent [MAE61], [WIL62], [MAE62]. However, no rational explanation between metal distribution and organic diluent chemical properties has been given [MAR69].

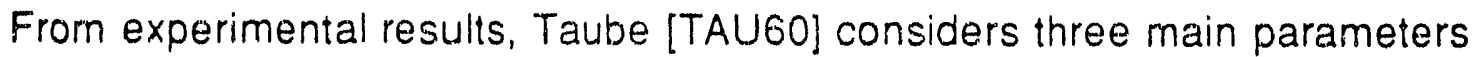
of the organic diluent affecting the metal distribution: the degree of extracted species aggregation; the dipole moment; and the dielectric constant of the solution. These parameters assume a uniform, unchanged dielectric constant 
and dipole. This has been shown to be invalid under experimental conditions [VDO61].

Concentration of the ligand affects aggregation, thereby changing the extractive behavior of the diluent. However, the concepts used do indicate a method to minimize diluent effects. If the amine ligand concentration is kept constant, and below the concentration where aggregation starts, the diluent effects can be reduced.

The amine structure also has a pronounced effect on the extraction of metals. Ignoring steric factors, the extractive power of amines increases from primary to secondary to tertiary. However, steric factors can change this order. In addition, steric factors generally change the basicity of the amines, which in turn affects the stability and polarity of the amine salts [MAR69].

The effect of alkyl chain length on extractive power has been studied in several systems [TRE64], [ZVY65]. Under normal experimental conditions, tertiary amines with eight member carbon chains extract best [MAR69]. Increase in chair length causes steric hindrance, while lower chain length decreases metal-ligand solubility in the organic phase.

The extraction of metals by the high molecular weight amines occurs by the formation of an ammonium salt. The reaction is:

$$
\mathrm{R}_{3} \mathrm{~N}+\mathrm{H}^{+}+\mathrm{A}^{-} \Leftrightarrow \mathrm{R}_{3} \mathrm{NH}^{+} \mathrm{A}^{-}
$$

where $R_{3} N$ is an organic soluble high molecular weight tentiary amine, $A$ - is an anionic metal complex, and $\mathrm{R}_{3} \mathrm{NH}^{+} \mathrm{A}^{-}$is the organic soluble extracted metalamine salt complex. This reaction readily occurs in acidic solutions and under certain conditions may take place in neutral or basic solutions [MARb9]. 
$\mathrm{HCl}$ solutions were the primary aqueous phases studied for these extraction systems. High oxidation state metals form anionic chloride species in $\mathrm{HCl}$ and readily extract with amines. The shape of extraction curves is similar for many extractable anionic chloride species. The lanthanides (trivalent actinide homologs) are only slightly extractable even at high amine concentrations [MAR69].

\section{2. $95 \mathrm{Zr}, 95 \mathrm{Nb}, 152 \mathrm{Eu}$, and $228 \mathrm{Th}$ Extractions}

In the experiments conducted, the high molecular weight amine extractants used were trihexylamine (THexA), triheptylamine (THepA), and triisooctylamine (TIOA). The information on these extractants is given in Table 13. The TIOA was purchased from Ames Laboratories and the other ligands were purchased from Aldrich Chemical Company. The TIOA was purified by vacuum distillation. The trihexylamine and triheptylamine were used as received.

\begin{tabular}{|lllll|}
\hline \multirow{5}{*}{ Amine } & Molecular & Formula & Density & Boiling Point \\
& Formula & Weight & $(\mathrm{g} / \mathrm{mL})$ & $\left({ }^{\circ} \mathrm{C}\right)$ \\
Trihexyl & $\left(\mathrm{CH}_{3}\left(\mathrm{CH}_{2}\right)_{5}\right)_{3} \mathrm{~N} 269.52$ & 0.794 & $263-265$ \\
Triheptyl & $\left(\mathrm{CH}_{3}\left(\mathrm{CH}_{2}\right)_{6}\right)_{3} \mathrm{~N}$ & 311.60 & 0.805 & $330-333$ \\
Triisooctyl & $\left(\mathrm{C}_{8} \mathrm{H}_{17}\right)_{3} \mathrm{~N}$ & 353.68 & 0.816 & $365-367$ \\
\hline
\end{tabular}

The aqueous phase was a $\mathrm{HCl}: \mathrm{HF}$ solution with different chloride, hydrogen ion or HF concentrations and contained the radioactive tracer. The tracers used were $95 \mathrm{Zr},{ }^{95} \mathrm{Nb},{ }^{152} \mathrm{Eu}$, and $228 \mathrm{Tr}$. The tracer stock solution was $12 \mathrm{M} \mathrm{HCl}$. In experiments on the organic diluent and the ligand concentration, the aqueous phase was $\mathrm{HCl}: \mathrm{HF}$ solution at different concentrations. Experiments with different chloride, hydrogen ion or HF concentrations were conducted with 
$1 \mathrm{M}$ amine ligand in benzene. The chloride concentration was adjusted with $\mathrm{LiCl}$. HF was added to prevent hydrolysis and evaporation of the tracer. Additionally, HF was removed from the aqueous phase to study the effect on tracer extraction.

Tracer loss during thin sample preparation was also studied. Tracer was extracted into the organic phase from concentrated acid solutions. From the organic phase, $20 \mu \mathrm{L}$ samples were placed on a glass cover slip on a hot plate. The samples were evaporated at various temperatures and counted versus a standard tracer to determine if the tracer was lost. These tests were conducted to determine if during evaporation to prepare samples for alpha spectroscopy, rutherfordium would be lost from a prepared sample.

The organic phase was the amine ligand in an organic solvent. To determine which organic solvent has the highest distribution coefficient, tracer extraction with TIOA was tested with methylisobutylketone (MIBK), hexanes, benzene and dichloromethane as solvents. Benzene was the diluent used for further tests with trihexylamine and triheptylamine. The amine concentrations were varied between 0.25 and $2.0 \mathrm{M}$.

The experiments were conducted with $500 \mu \mathrm{L}$ each of an aqueous and an organic phase. The aqueous phase contained $50 \mu \mathrm{L}$ of tracer solution. The two phases were mixed for 2 minutes, centrifuged for 30 seconds, separated and counted for activity. Microscale experiments, using $20 \mu \mathrm{L}$ of each phase, were also conducted. These experiments verified the validity of the extractions on a microscale. From counts of the aqueous phase and the organic phase the distribution coefficients ( $K_{d}=$ (cts organic/volume organic)/(cts aqueous/volume aqueous)), or percent extraction were obtained. 


\section{3. $95 \mathrm{Zr}, 95 \mathrm{Nb}, 152 \mathrm{Eu}$, and $228 \mathrm{Th}$ Results}

The initial experiments examined the effect of the organic diluent on tracer extraction (Figures 17-19). The organic diluents studied were MIBK, benzene, hexanes, and dichloromethane. The ligand used in these experiments was TIOA. The ligand concentration was varied between $0.2 \mathrm{M}$ and $1.0 \mathrm{M}$. The aqueous phase was $12 \mathrm{M} \mathrm{HCl:0.02} \mathrm{M} \mathrm{HF}$. The volume of each phase was 500 $\mu \mathrm{L}$, as was the case in all the experiments unless otherwise noted. ${ }^{95} \mathrm{Zr},{ }^{95} \mathrm{Nb}$ and ${ }^{152}$ Eu tracers were used.

The results from the experiments with different diluents dictated the use of benzene. The $95 \mathrm{Zr}$ experiment (Figure 17) showed benzene to be the diluent that gave the largest extraction, especially at high ligand concentrations. In addition, benzene does not enhance the extraction of 152Eu (Figure 19), as did the other solvents. MIBK is the best diluent for the extraction of ${ }^{95} \mathrm{Nb}$ (Figure 18). This result is consistent with the ability of MIBK to extract pentavalent metals from concentrated $\mathrm{HCl}$.

After deciding upon the use of benzene as the organic diluent, the three amine ligands were studied for tracer extraction (Figures 20-22). The ligands, trihexylamine, triheptylamine and TIOA, were diluted with benzene to give final concentrations of $0.2 \mathrm{M}$ to $2.0 \mathrm{M}$. The aqueous phase was $12 \mathrm{M} \mathrm{HCl:0.02} \mathrm{M} \mathrm{HF}$. $95 \mathrm{Zr}, 95 \mathrm{Nb},{ }^{152} \mathrm{Eu}$, and $228 \mathrm{Th}$ tracers were used.

The results for different amine ligand concentrations show TIOA to be the best extractant. This result agrees with findings reported in the literature [MAR69]. The shape of the $95 \mathrm{Zr}$ extraction curve is similar for the three amine ligands. $95 \mathrm{Zr}$ extraction reaches a maximum at $1.0 \mathrm{M}$ ligand concentration. Above this concentration, aggregation occurs and inhibits zirconium extraction. Europium extraction is minimal for the three amine ligands, demonstrating the abiliiy of these ligands to separate rutherfordium from the trivalent actinides. 
Figure 17. Diluent Effect on $95 \mathrm{Zr}$ Extraction with TIOA

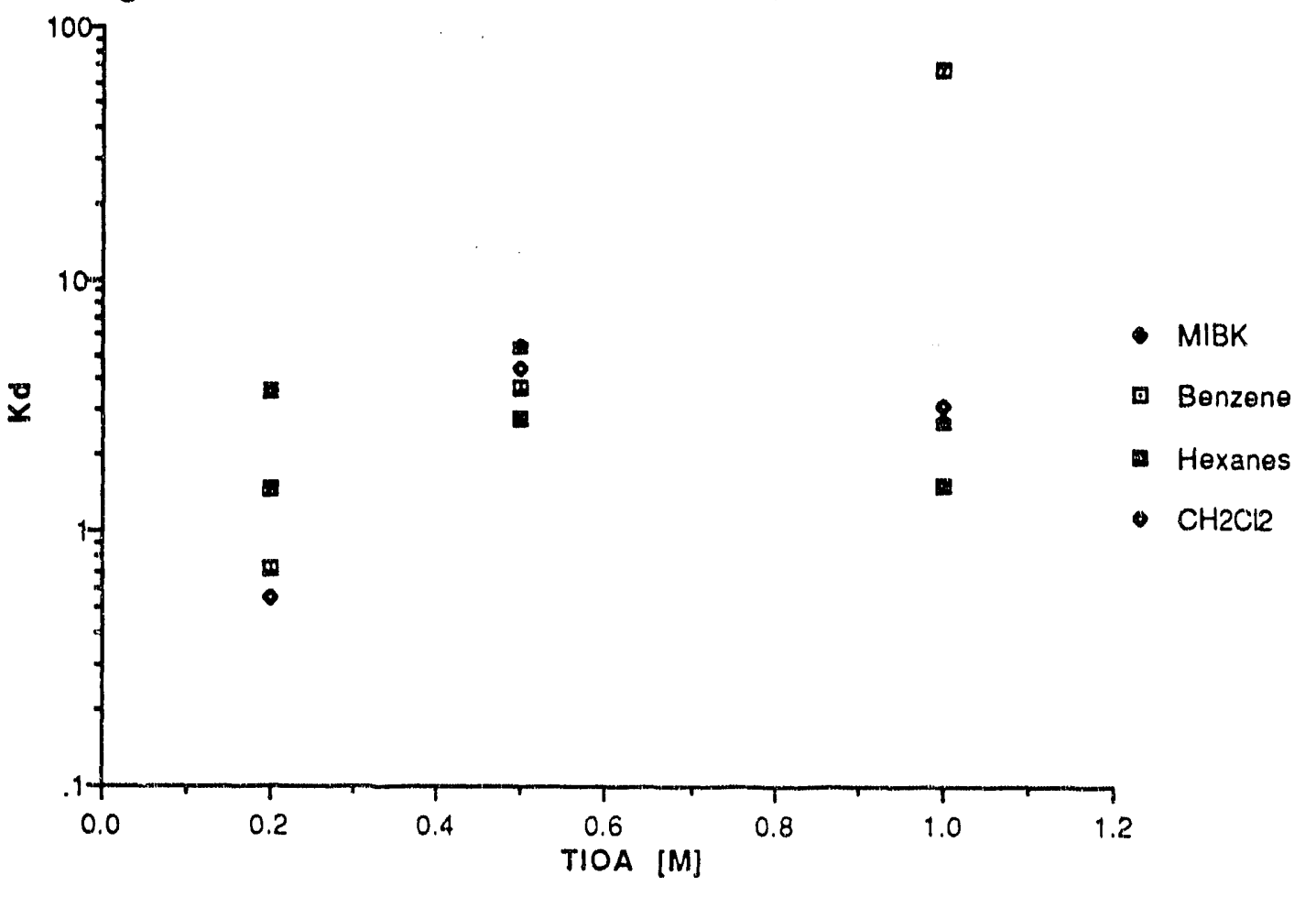

Figure 18. Diluent Effect on ${ }^{95} \mathrm{Nb}$ Extraction with TIOA

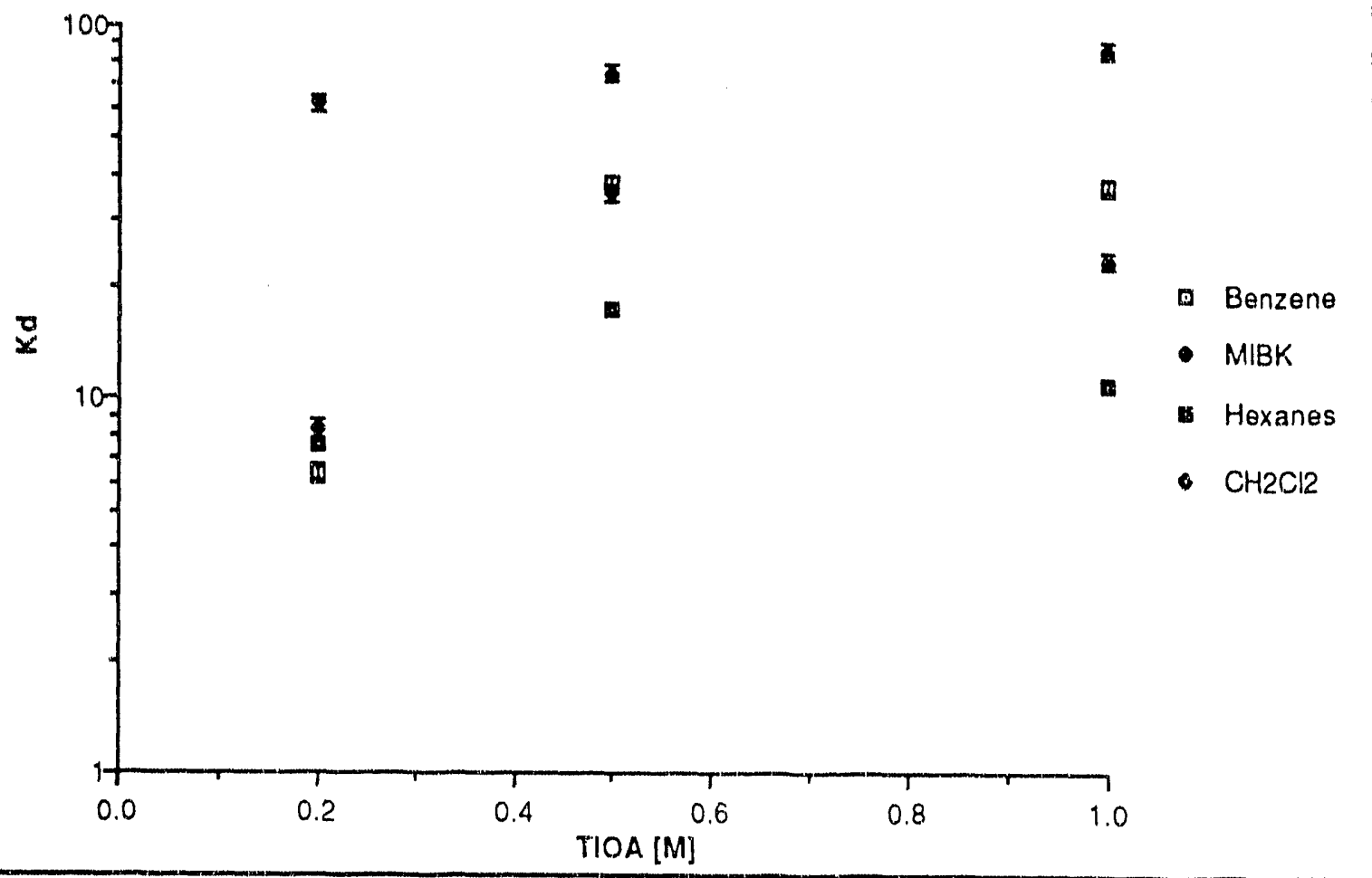


Figure 19. Diluent Effect on ${ }^{152} \mathrm{Eu}$ Extraction with TIOA

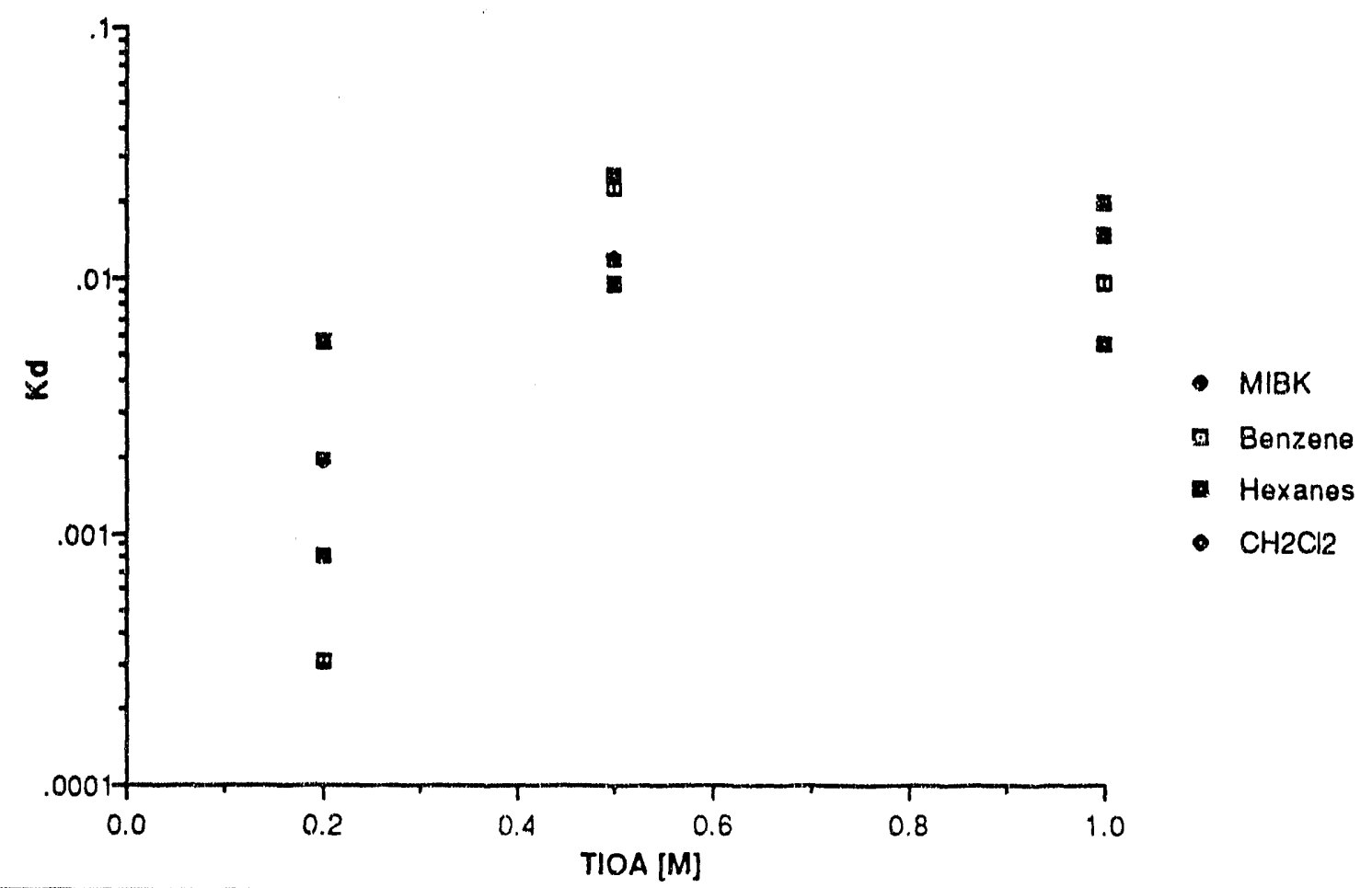

Figure 20. Effect of [Trihexylamine] on Tracer Extraction

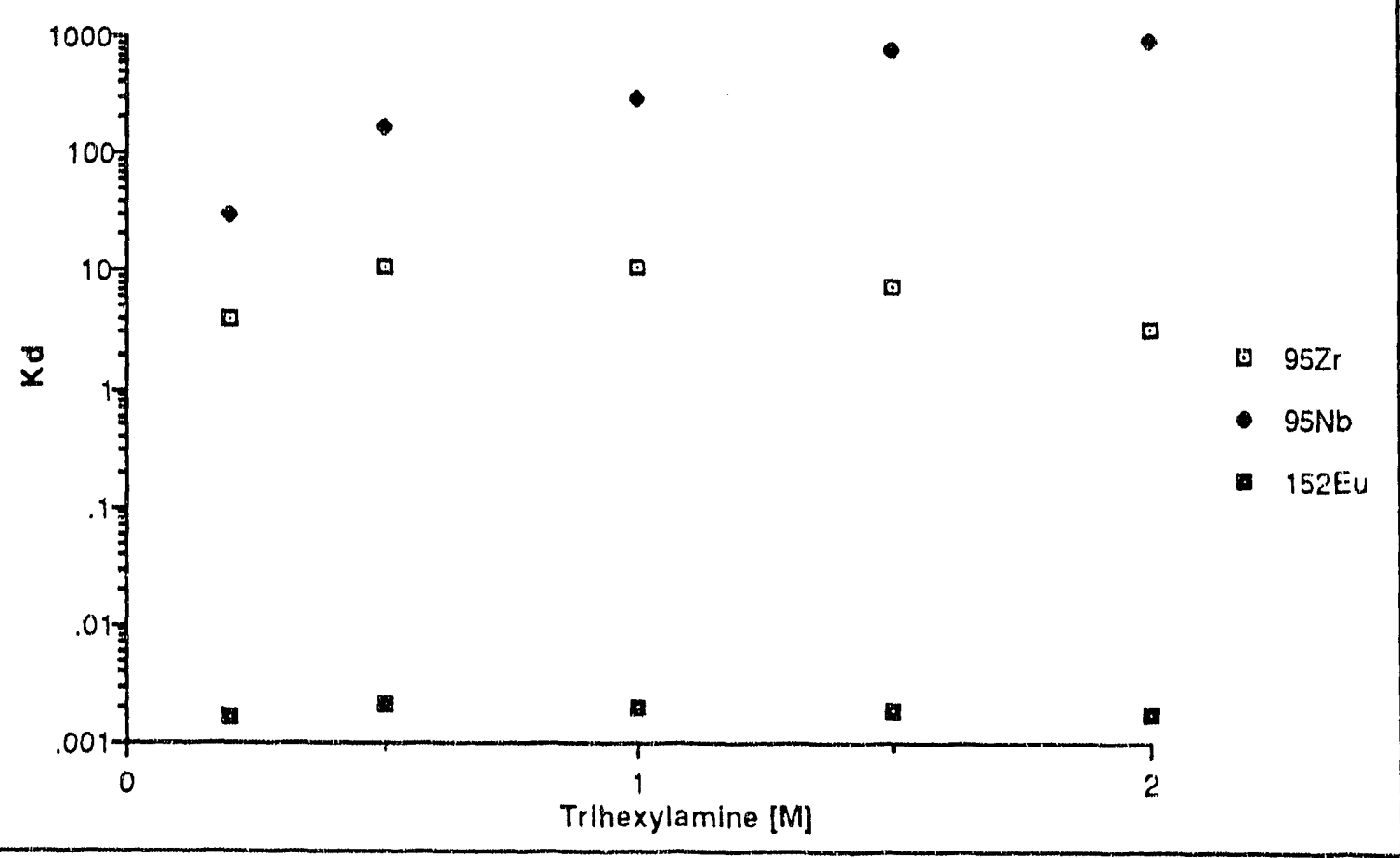


Figure 21. Effect of [Triheptylamine] on Tracer Extraction

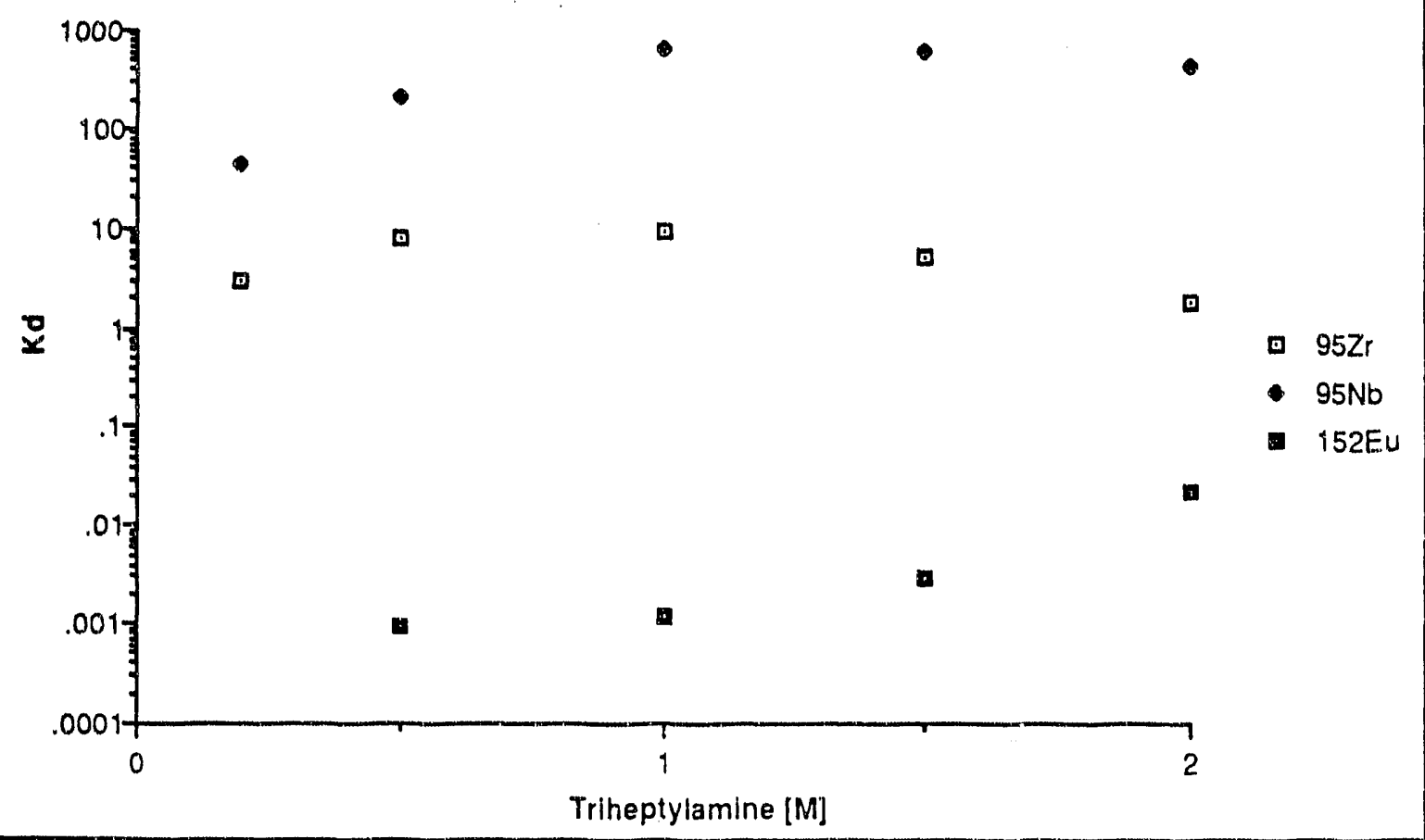

Figure 22. Effect of [TIOA] on Tracer Extraction

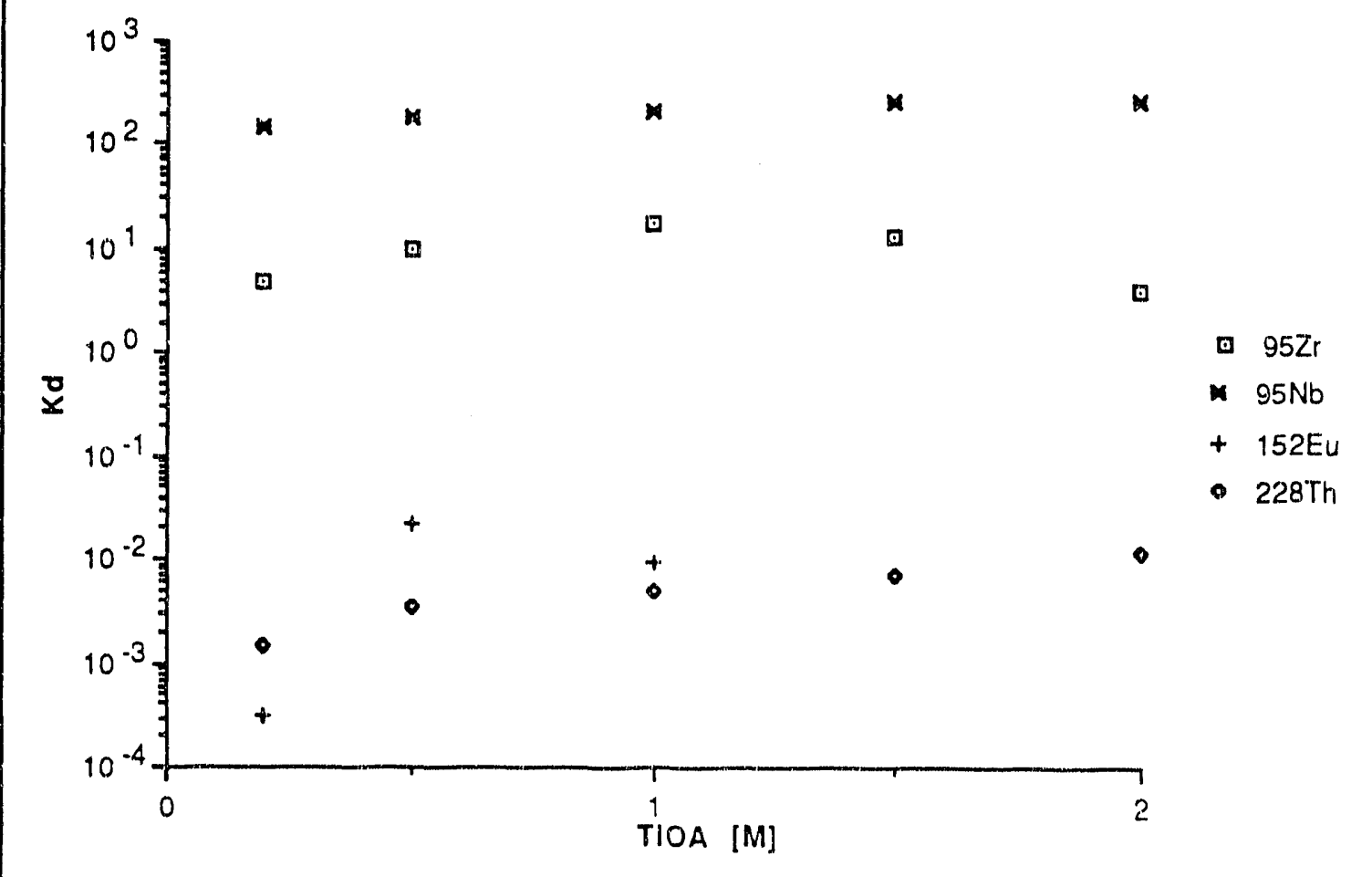


TIOA is shown not to extract tetravalent thorium (Figure 22). This result is expected, since Equation 1 requires the formation of an anionic metal chloride complex. Thorium does not form anionic species even in high chloride concentration, and therefore is not extracted. This fact can be used to give further proof that rutherfordium is a Group 4 metal. If rutherfordium is extracted by TIOA, it is chemically similar to zirconium. If rutherfordium is not extracted by TIOA, then it would be chemically similar to thorium.

The extraction curve for niobium differs for the zirconium extraction curve. The extraction of niobium generally increases as the amine ligand concentration increases. This implies that the aggregation effects, which limit the zirconium extraction, have little effect on niobium. The higher valent state of niobium makes aggregation more difficult by preventing the formation of highly negative anionic species. Zirconium hexachloride has $2 \cdot$ charge, while niobium hexachloride has only a 1-charge. With the same coordination number for the two metals, zirconium anion is able to accept more cationic amine species. This fact accounts for the high aggregation of the zirconium-amine complex compared to the niobium-amine complex.

The next experiment conducted was on the effect of chloride concentration on tracer extraction (Figures 23 and 24). These experiments were designed to determine if a chloride salt solution could be used for extractions. The chloride concentration of the aqueous phase was varied with constant $8 \mathrm{M} \mathrm{H}^{+}: 0.02 \mathrm{M}$ $\mathrm{HF}$. The organic phase was $1 \mathrm{M}$ ligand in benzene. All amine ligands were studied. The chloride concentration was varied from $8 \mathrm{M}$ to $12 \mathrm{M}$.

The effect of chloride concentration on extraction again showed TIOA to be the best amine extractant for zirconium. The zirconium distribution increased as a function of chloride concentration for all three amine extractants studied. This effect is due to the increased anionic zirconium chloride species formation at 
higher chloride concentration. The niobium extraction shows a plateau starting at $10 \mathrm{M}$ chloride concentration. Up to this point, the extraction increases as a function of chloride concentration. This observation indicates the formation of anionic niobium chloride species reaches a saturation point at $10 \mathrm{M}$ chloride concentration. After this point, the extraction remains constant.

The effect of hydrogen ion concentration on tracer extraction was studied (Figures 25 and 26). These experiments were designed to examine if a weakly acidic solution could be used for extractions if a high chloride concentration was maintained. The hydrogen ion concentration in the aqueous phase was varied and extracted with $12 \mathrm{M} \mathrm{Cl}: 0.02 \mathrm{M} \mathrm{HF}$ with TIOA and $10 \mathrm{M} \mathrm{Cl}^{-}: 0.02 \mathrm{M} \mathrm{HF}$ with trihexylamine and triheptylamine. The organic phase was $1 \mathrm{M}$ ligand in benzene. The hydrogen ion concentration was varied between $7 \mathrm{M}$ and $10 \mathrm{M}$ with trihexylamine and triheptylamine and between $8 \mathrm{M}$ and $12 \mathrm{M}$ with TIOA.

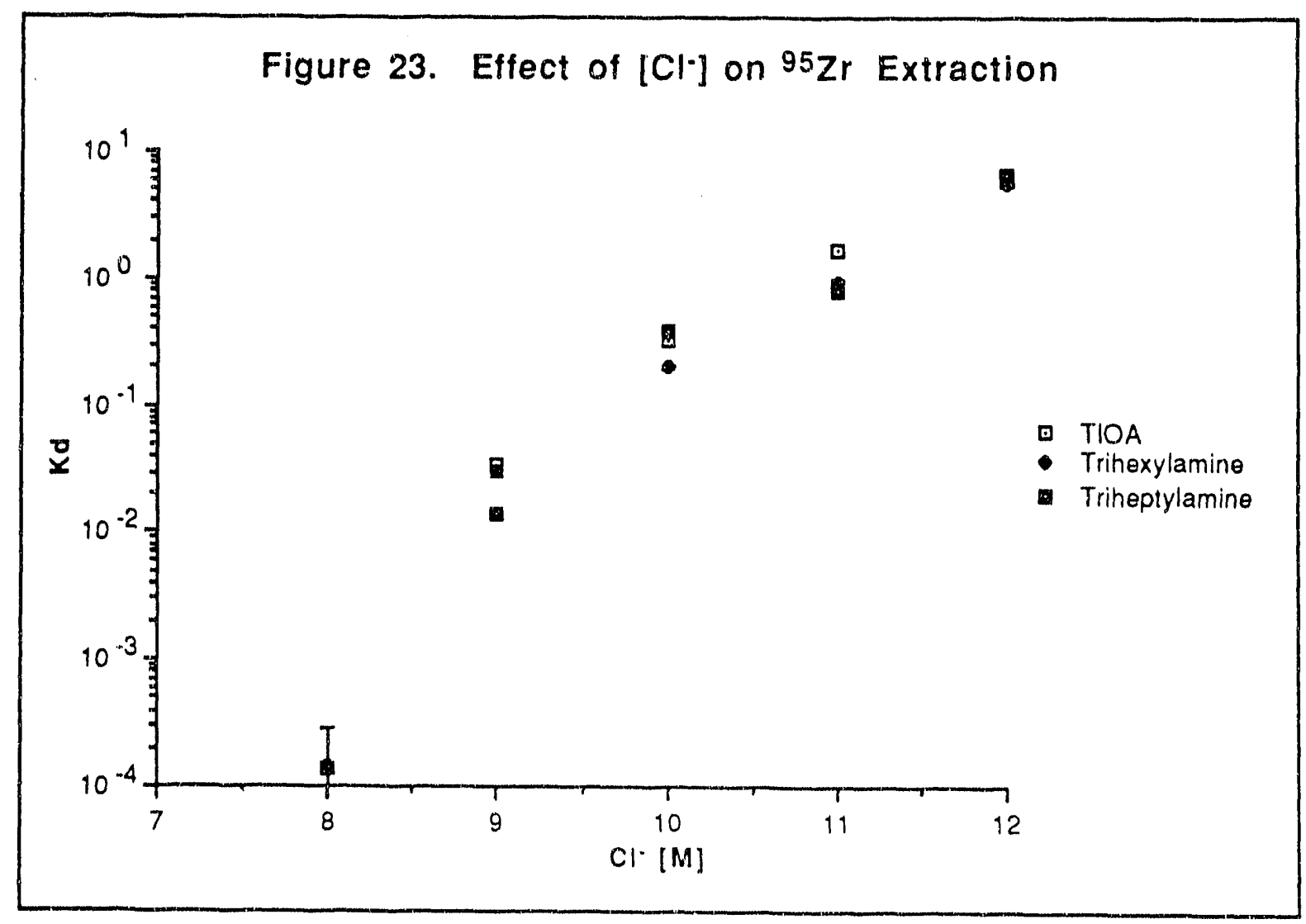



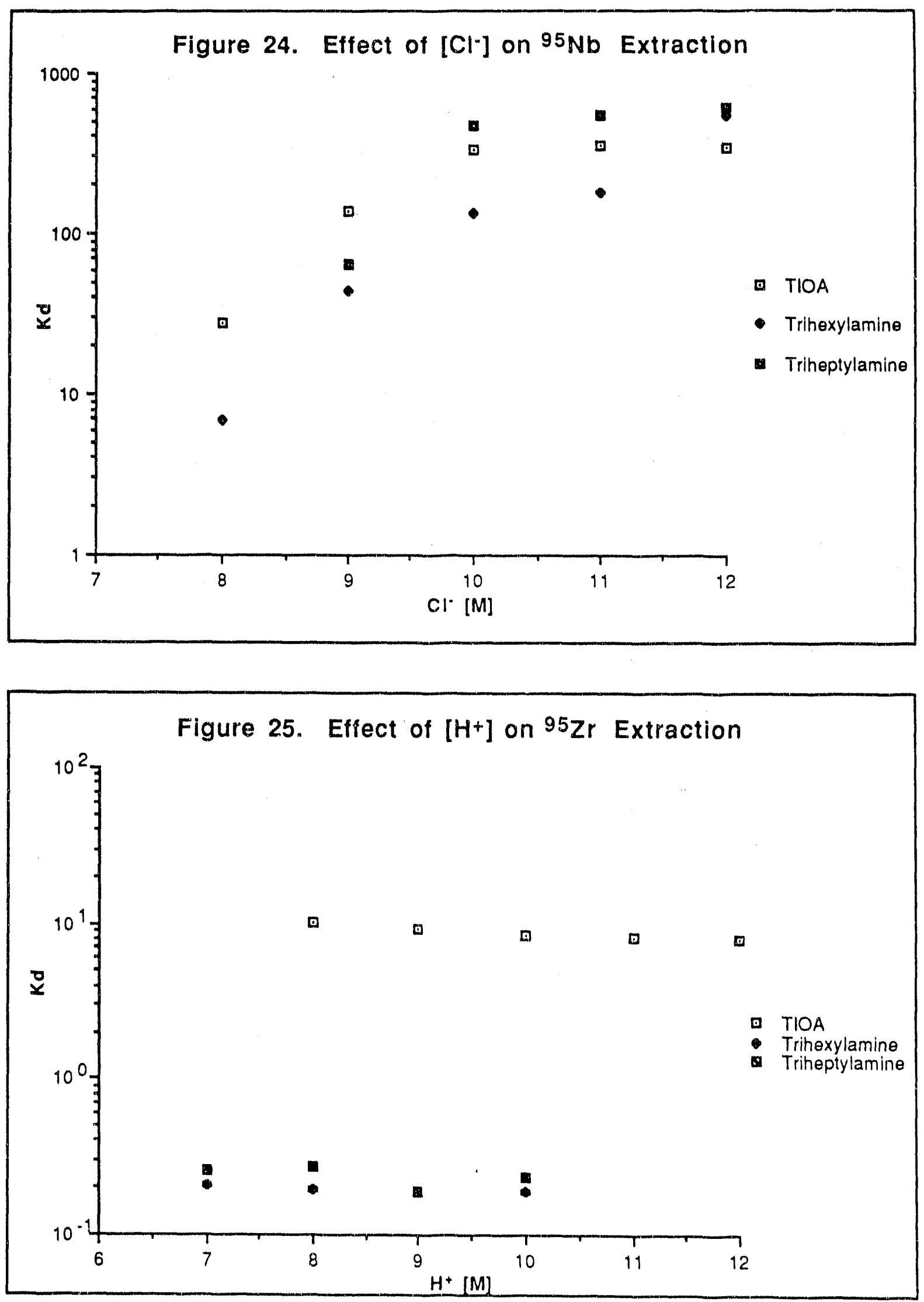


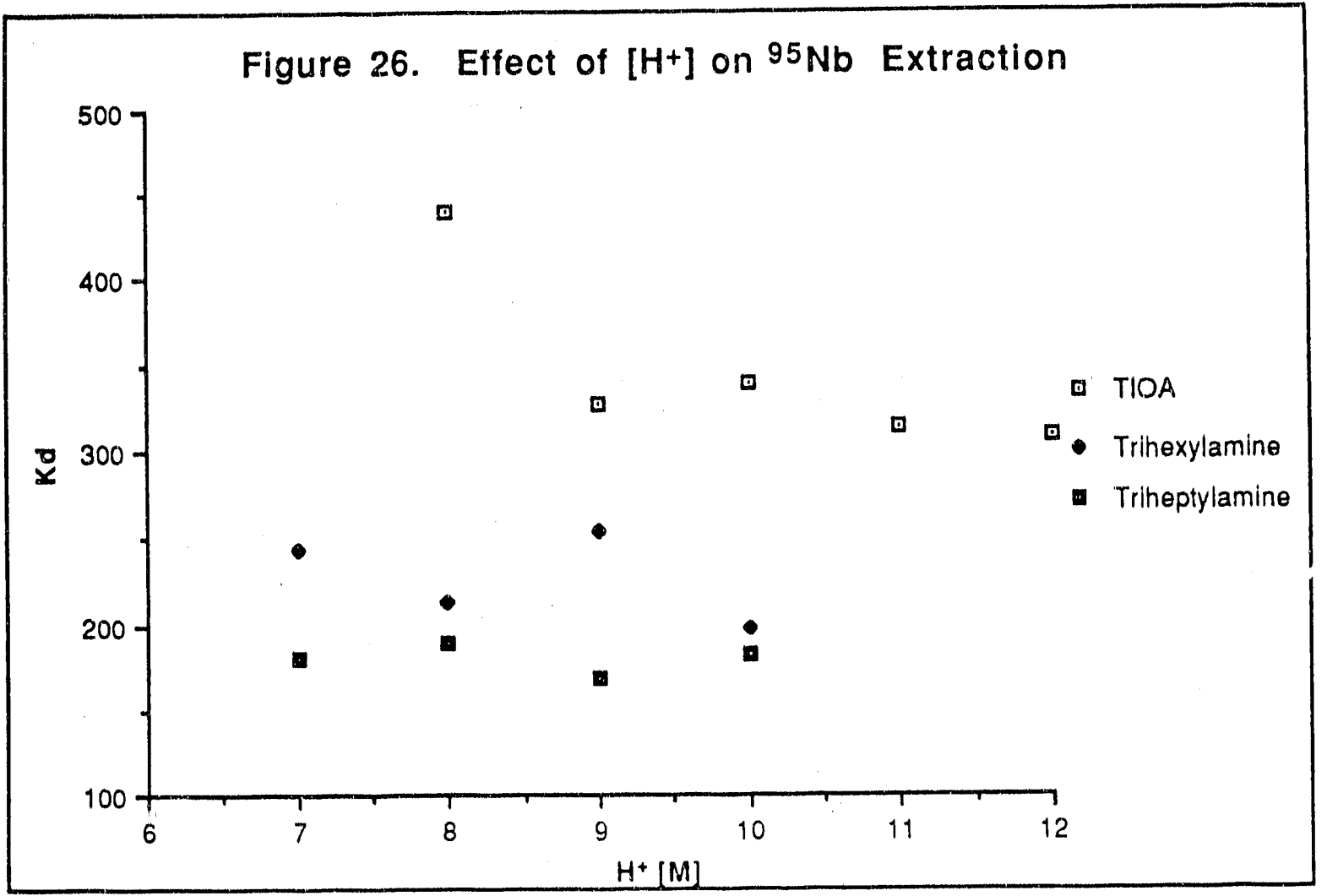

The results indicate a minima, nydrogen ion effect over the examined range. Both Figures 25 and 26 show little change in $K_{d}$ with an increase in hydrogen ion concentration. This effect is expected in the hydrogen ion region studied. The amine ligands should be fully protonated even at $7 \mathrm{M}$ hydrogen ion concentration, the lowest concentration examined in this experiment. An increase in $K_{d}$ would have indicated an increase in amine protonation, which would be unexpected in this region.

Experiments on zirconium and niobium extraction as a function of $\mathrm{HF}$ concentration were conducted (Figures 27 and 28). These experiments showed that $\mathrm{HF}$ inhibited tracer extraction. The aqueous phase was $10 \mathrm{M} \mathrm{HCl}$ with different HF concentrations. The organic phase was $1 \mathrm{M}$ ligand in benzene. All three amine ligands were studied. 

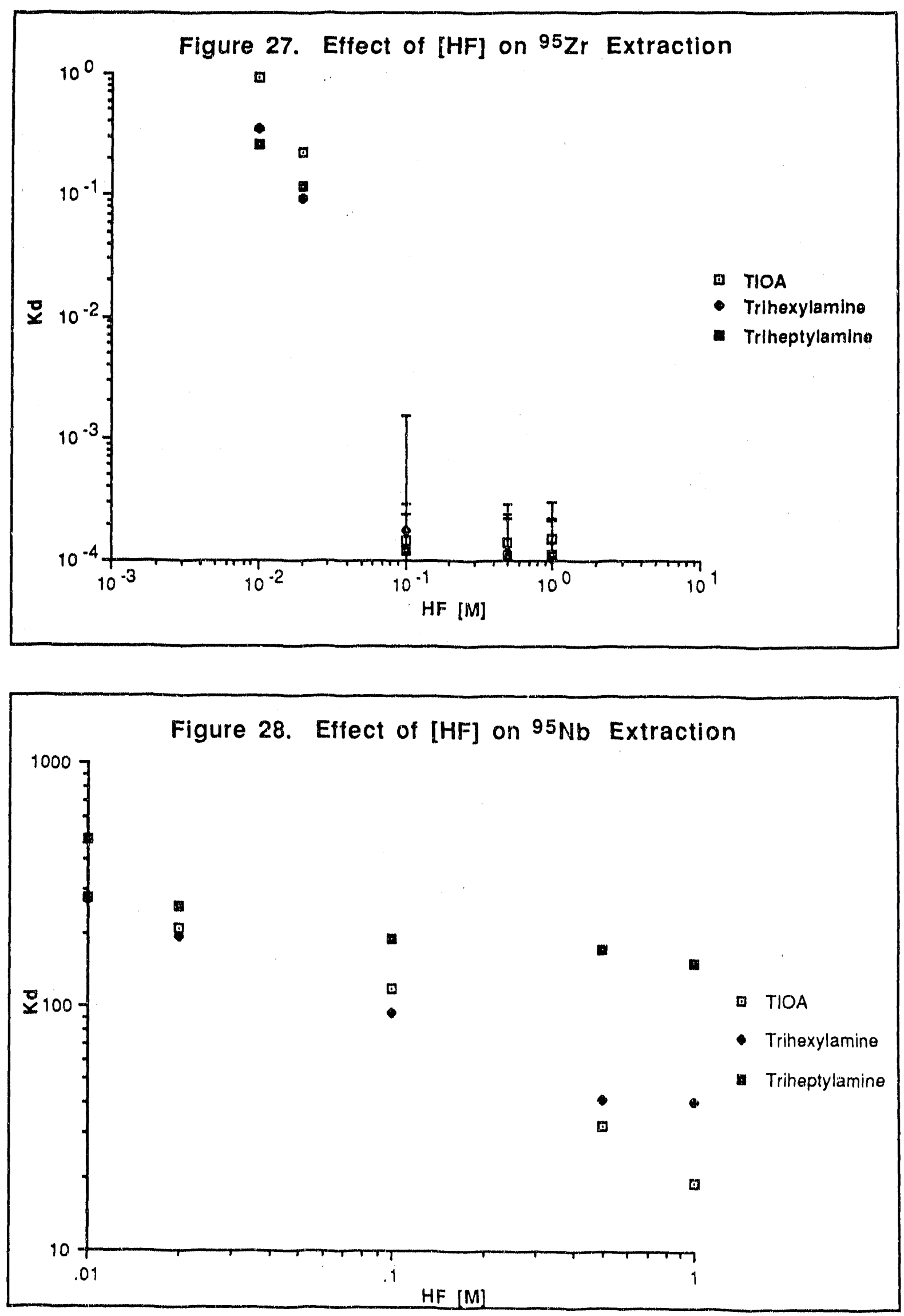
These results displayed a decrease in tracer extraction as a function of increasing HF concentration. The increase in fluoride concentration will increase the formation of a metal-fluoride species. The fluoro species of zirconium is insoluble in the organic phase and not extracted by amines. In addition, HF competes with anionic metal species for complexation with the cationic amines. The reason for the competition from HF is its ability to dimerize and form the $\mathrm{HF}_{2}$ - anion. The extracted $\mathrm{HF}$ amine species is generally $\left(\mathrm{R}_{3} \mathrm{NH}_{n} \mathrm{~F}_{n+1}\right)^{1-}[\mathrm{HER} 45]$. The HF extraction is shown to compete with niobium extraction (Figure 28). Even in the dilute concentrations used in these experiments, HF has the ability to hinder zirconium extraction (Figure 27).

The next experiments studied the effect of temperature on tracer evaporation during thin sample formation (Figures 29 and 30 ). Thin sample formation is unnecessary for gamma spectroscopy. It is, however, required for alpha spectroscopy, which is used to detect rutherfordium. In these experiments, the aqueous phase was $12 \mathrm{M} \mathrm{HCl:0.02} \mathrm{M} \mathrm{HF}$ and the organic phase was $1 \mathrm{M}$ ligand in benzene.

The evaporation studies show little difference between tracer loss during evaporation at $250^{\circ} \mathrm{C}$. However, as the temperature was increased, differences appeared in the amount of tracer loss. TIOA performed the best, having the overall lowest tracer loss during evaporation of the organic phase. Trihexylamine generally had the highest tracer loss. TIOA has the highest boiling point of the amines studied (Table 13). This may account for the low loss of tracer compared to the other amines. As the temperature was increased, the amines with lower boiling points burned more completely. This created more aerosols, increasing the amount of tracer loss. 

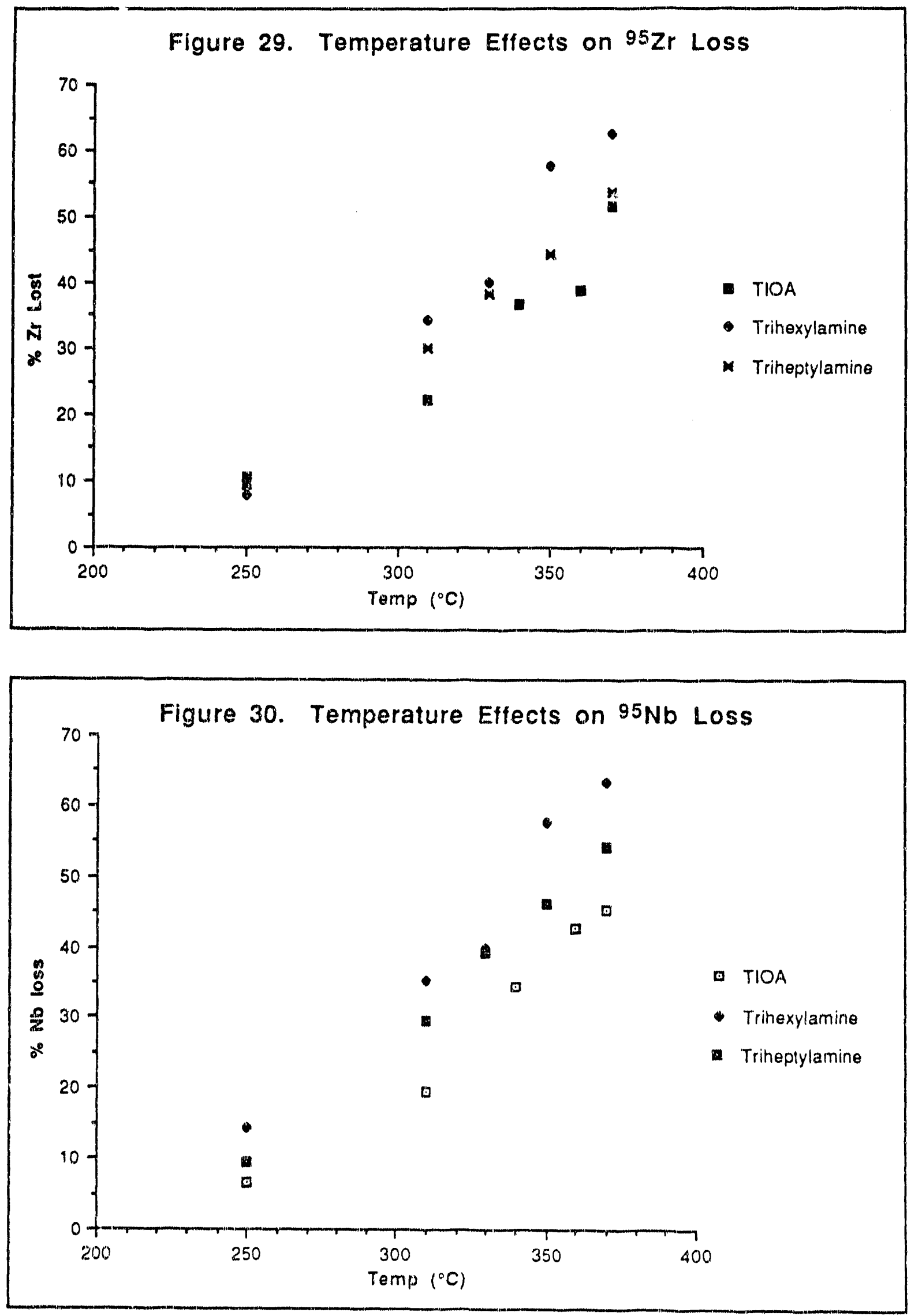
The experiment conducted with the various amines indicated that TIOA gave the best results for zirconium extraction. In addition, the HF experiments with zirconium pointed out the need to examine aqueous systems without HF (Figure 27). Therefore, the following series of experiments was conducted to examine the difference between tracer extraction with and without HF.

The effect of TIOA concentration on tracer extraction is presented in Figure 31. The aqueous phase was $12 \mathrm{M} \mathrm{HCl}$ and the TIOA concentration in benzene was varied from $0.1 \mathrm{M}$ to $2.0 \mathrm{M}$. This result can be directly compared with Figure 22 (varied [TIOA] M with HF), and shows increased zirconium extraction when HF is not in the aqueous phase. This conclusion is supported by Figure 27, which shows HF decreases tracer extraction. The zirconium extraction curve is similar for both experiments, indicating no change in the TIOA stoichiometry of the complex.

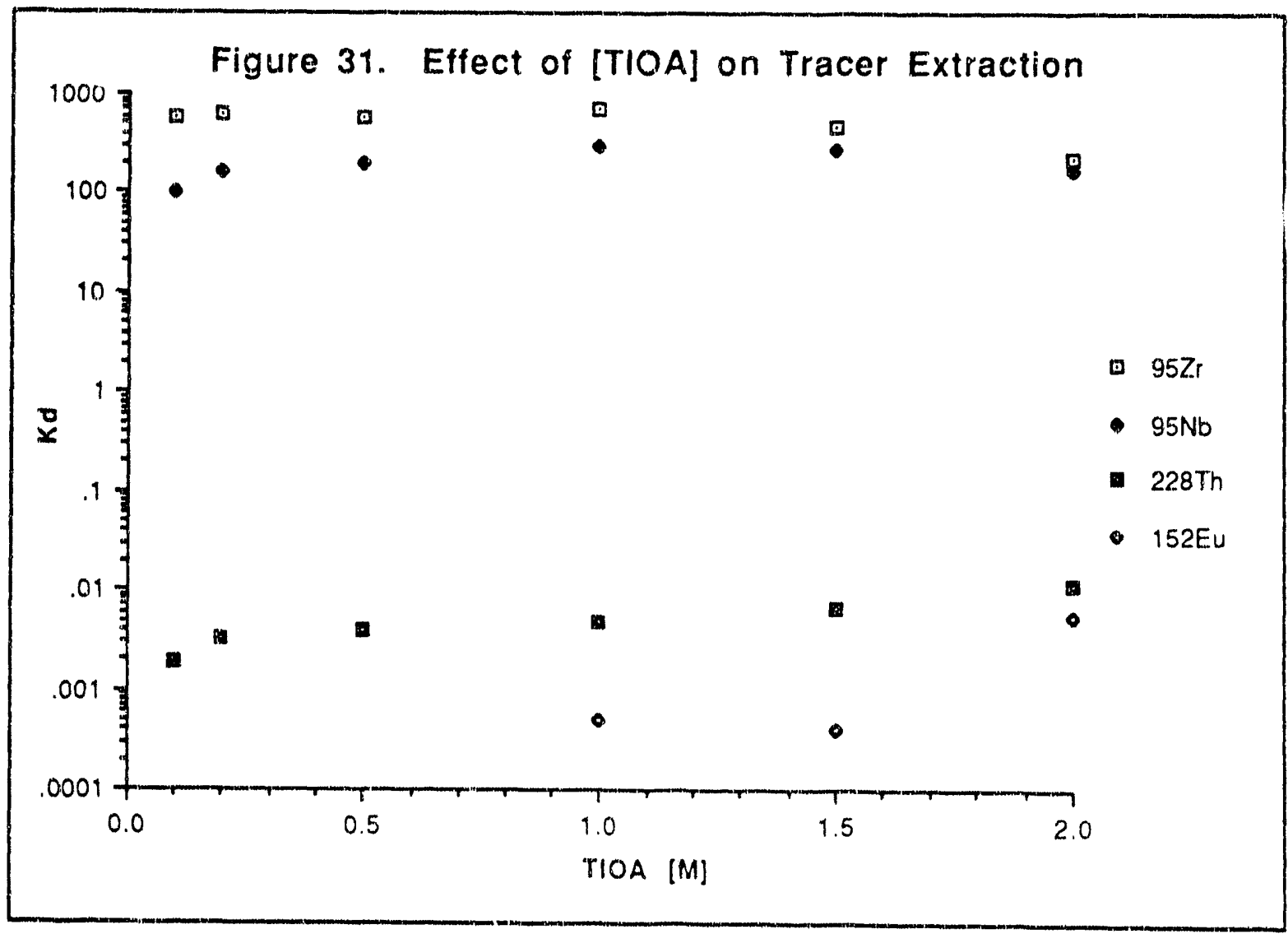


Further experiments were conducted in which the $\mathrm{HCl}$ concentration was varied. In Figure 32, the aqueous phase was different $\mathrm{HCl}$ concentrations and in Figure 33, the aqueous phase was $0.02 \mathrm{M} \mathrm{HF}$ with $\mathrm{HCl}$ concentrations from 2 $M$ to $12 \mathrm{M}$. In both experiments the organic phase was $1 \mathrm{M}$ TIOA in benzene. The results show that over the $\mathrm{HCl}$ range of $2 \mathrm{M}$ to $12 \mathrm{M}, 0.02 \mathrm{M} \mathrm{HF}$ inhibited tracer extraction. At $12 \mathrm{M} \mathrm{HCl}$, zirconium extraction without $\mathrm{HF}$ was greater by over 1 order of magnitude, and at $2 \mathrm{M} \mathrm{HCl}$, the distribution coefficient was greater by over two orders of magnitude. This indicates that the formation of a zirconium fluoride complex becomes more pronounced at lower chloride concentrations, thus inhibiting extraction.

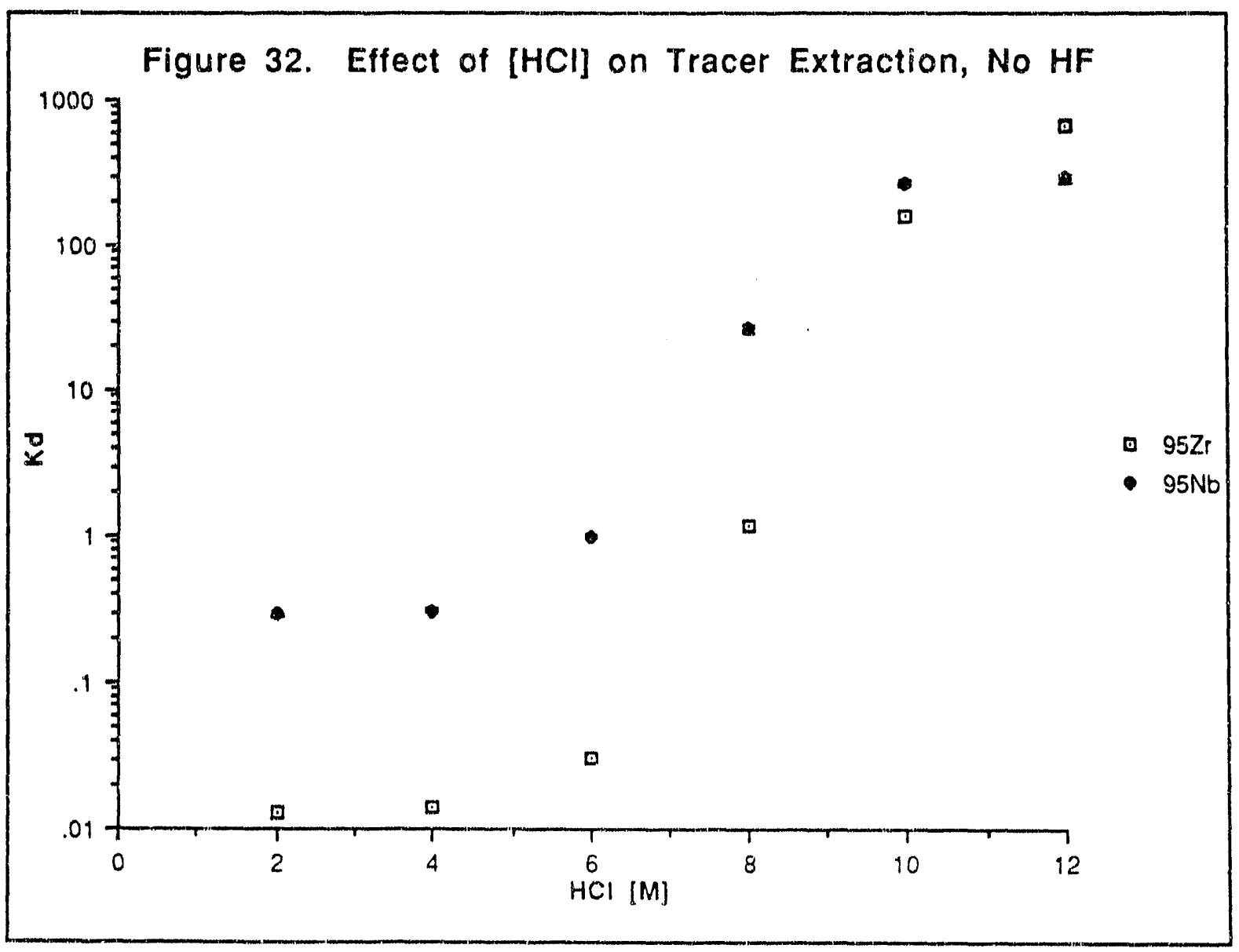




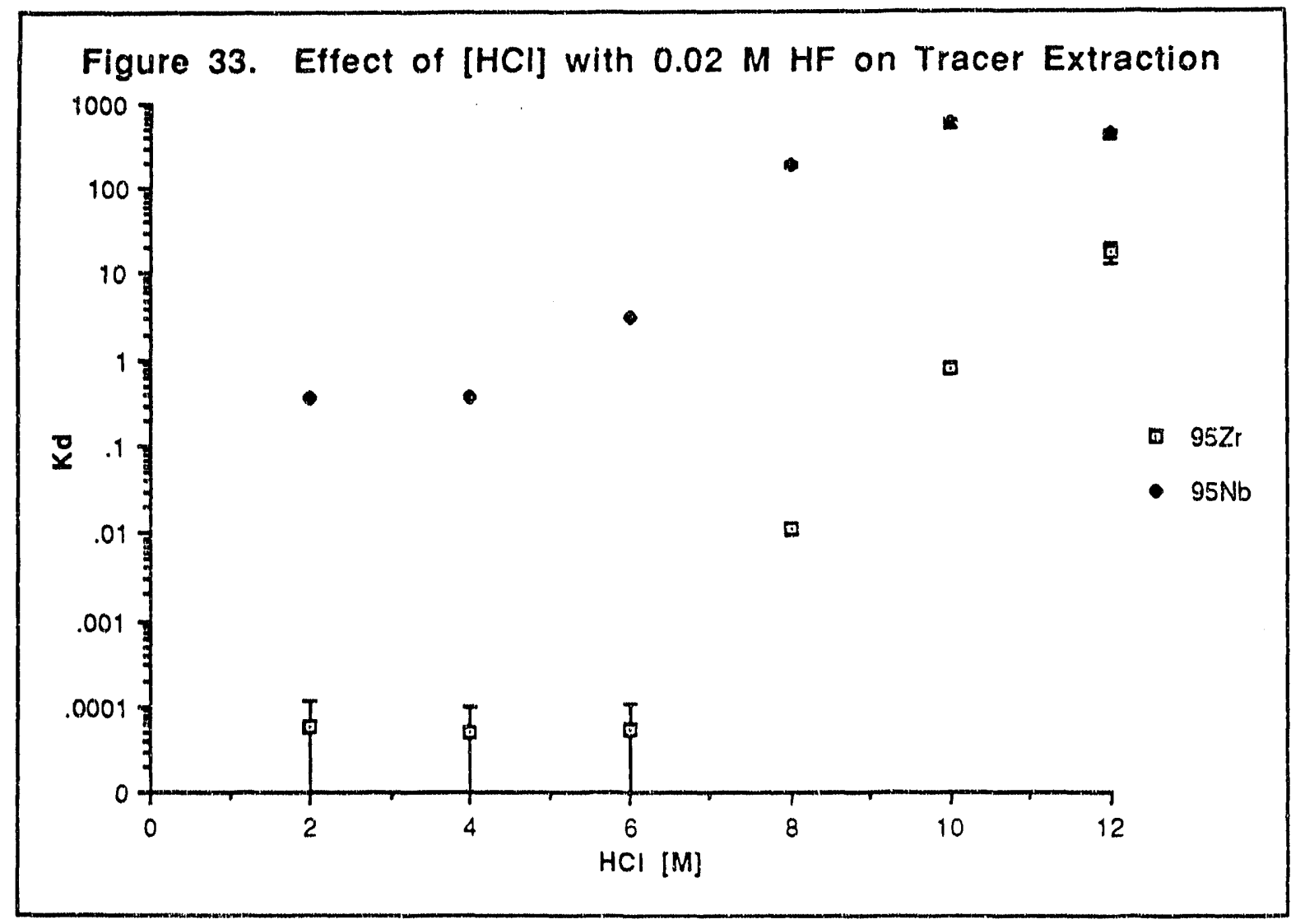

In order to fully determine if HF could be eliminated from the aqueous phase, zirconium losses due to evaporation had to be studied. In these experiments, the aqueous phase was $12 \mathrm{M} \mathrm{HCl}$ and the TIOA concentration in benzene was varied from $0.1 \mathrm{M}$ to $1.0 \mathrm{M}$ to determine its effect upon tracer loss. The results from these thin sample formation experiments are presented in Figure 34.

The results, compared with Figure 29, show no real difference between zirconium loss on evaporation from $1 \mathrm{M}$ TIOA. This indicates that the lack of HF in the aqueous phase will not effect the amount of tracer loss in sample evaporation. Furthermore, the results indicate a dramatic decrease of tracer loss when a TIOA concentration less than $1 \mathrm{M}$ is used. This is especially true at higher temperatures. 


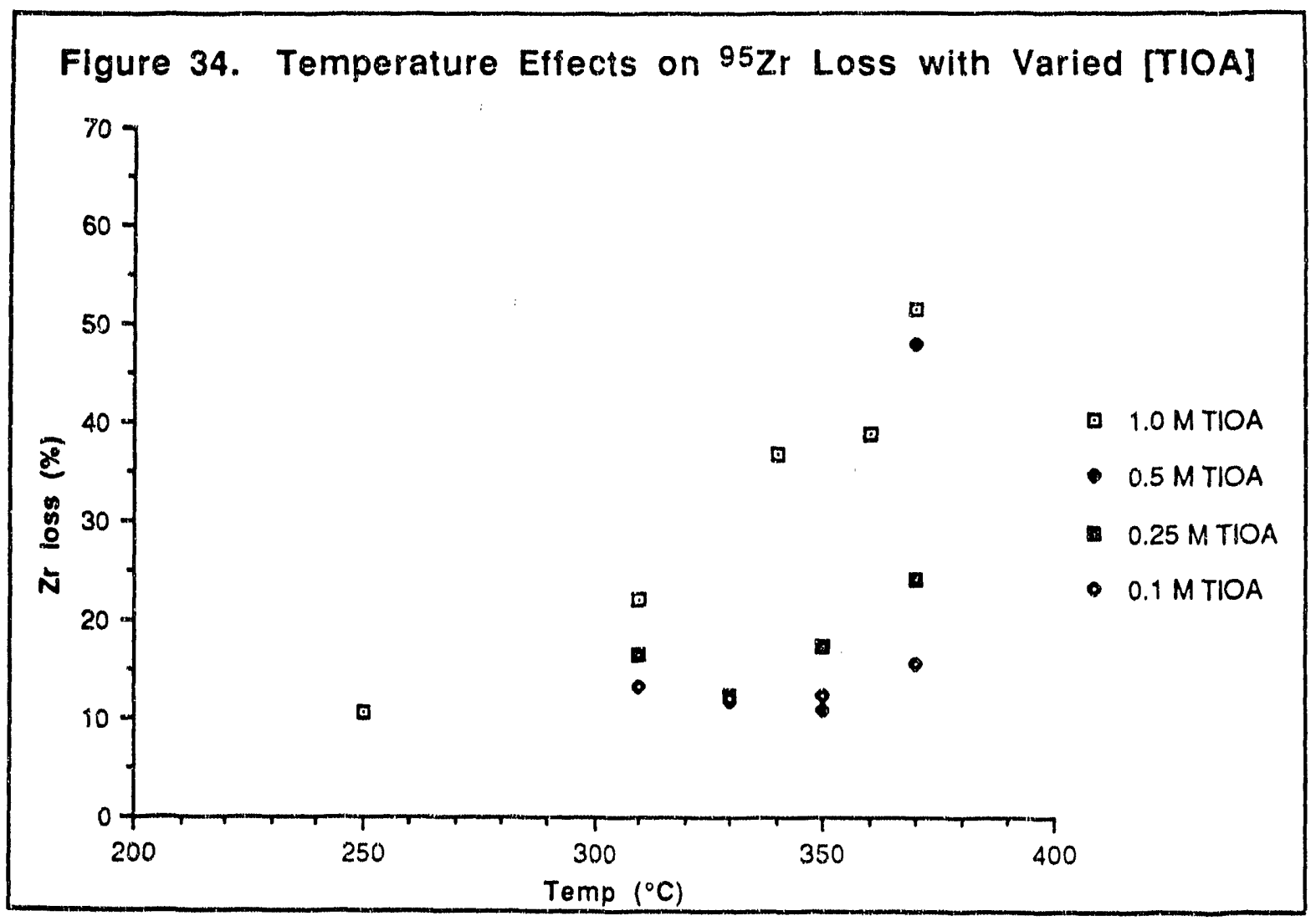

These tracer extraction studies with TIOA in a non-HF aqunous solution gave better results than extractions with an aqueous phase containing HF. Therefore, it was decided to use an $\mathrm{HCl}$ aqueous phase without $\mathrm{HF}$ for rutherfordium extraction studies. Further experiments on chloride and hydrogen ion concentration effects with TIOA were studied.

For the experiments with varied chloride concentrations, the organic phase was $1 \mathrm{M}$ TIOA in benzene and the hydrogen ion concentration in the aqueous phase was $1 \mathrm{M}$ (Figure 35) or $7 \mathrm{M}$ (Figure 36). For the studies of different hydrogen ion concentrations, the chloride concentration was held constant at 9 $M$ (Figure 37) and $12 M$ (Figure 38). The organic phase was $1 M$ TIOA in benzene. The chloride and hydrogen ion ranges differed compared to the previous experiments. The y $M$ acid phase was examined to determine if these extractions may be useful with instruments sensitive to strong acid. 
Figure 35. Effect of [Cl-] at $1 \mathrm{M} \mathrm{H}^{+}$on Tracer Extraction with TIOA

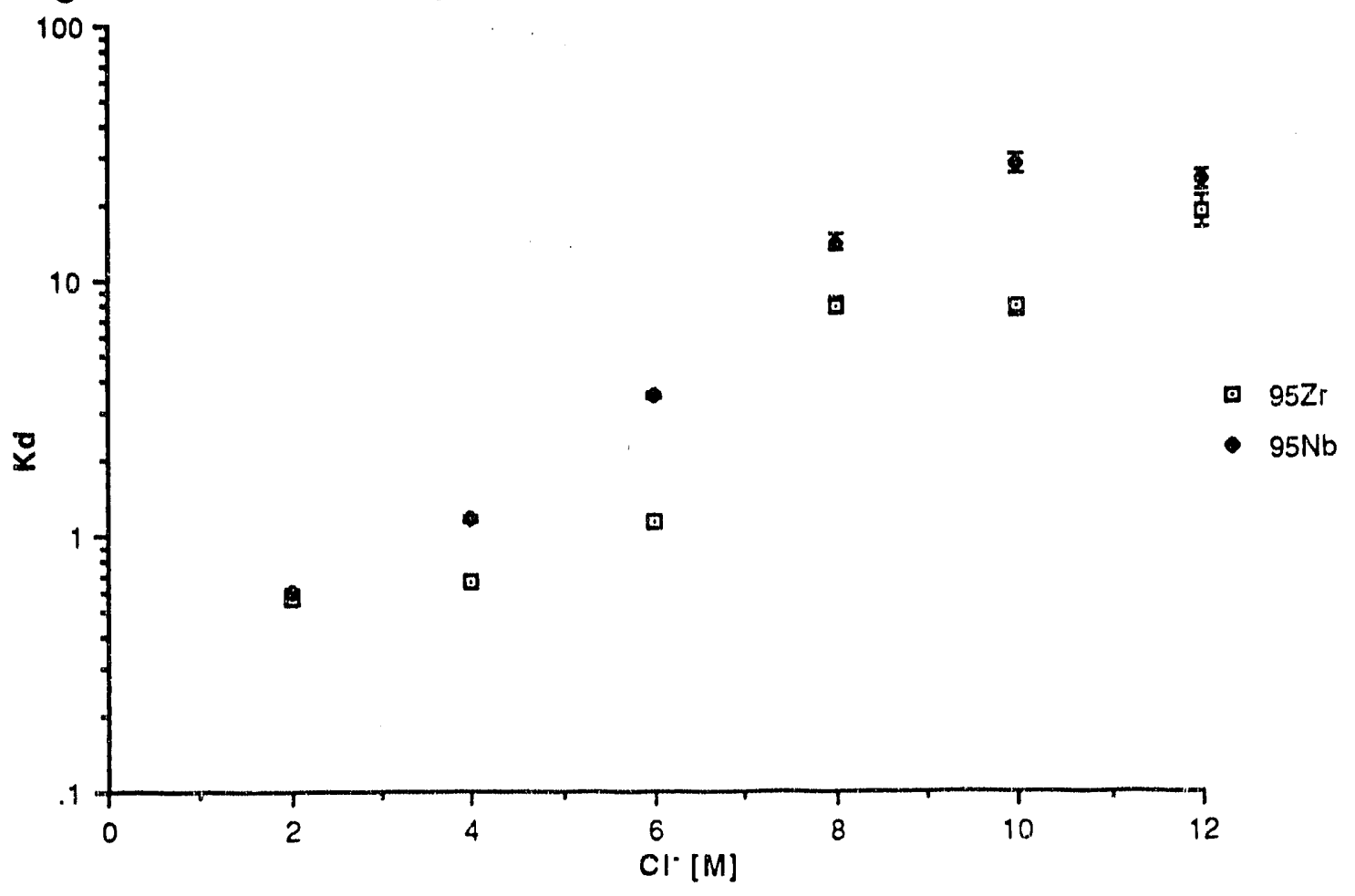

Figure 36. Effect of $\left[\mathrm{Cl}^{-}\right]$at $7 \mathrm{M} \mathrm{H}^{+}$on Tracer Extraction with TIOA

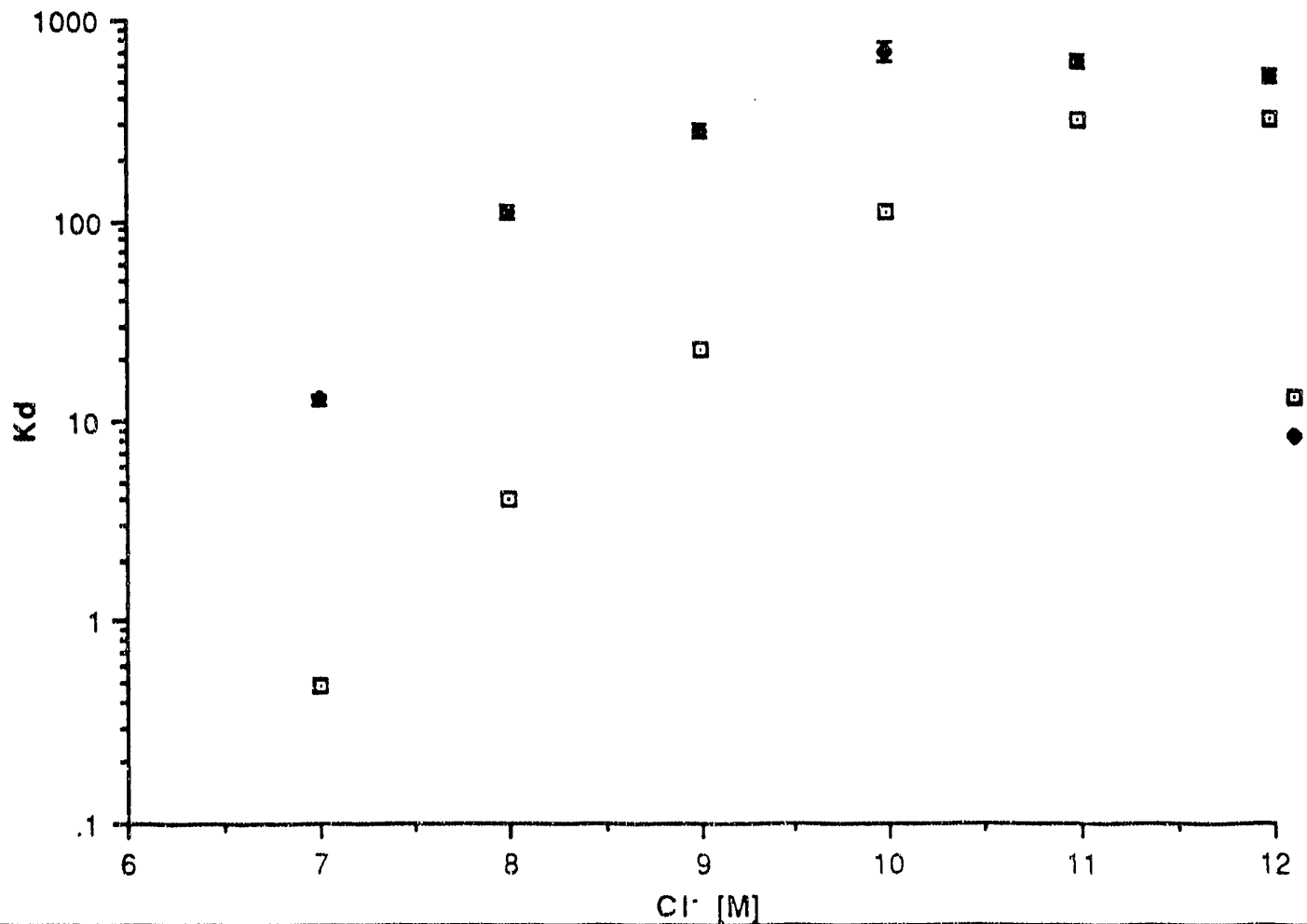




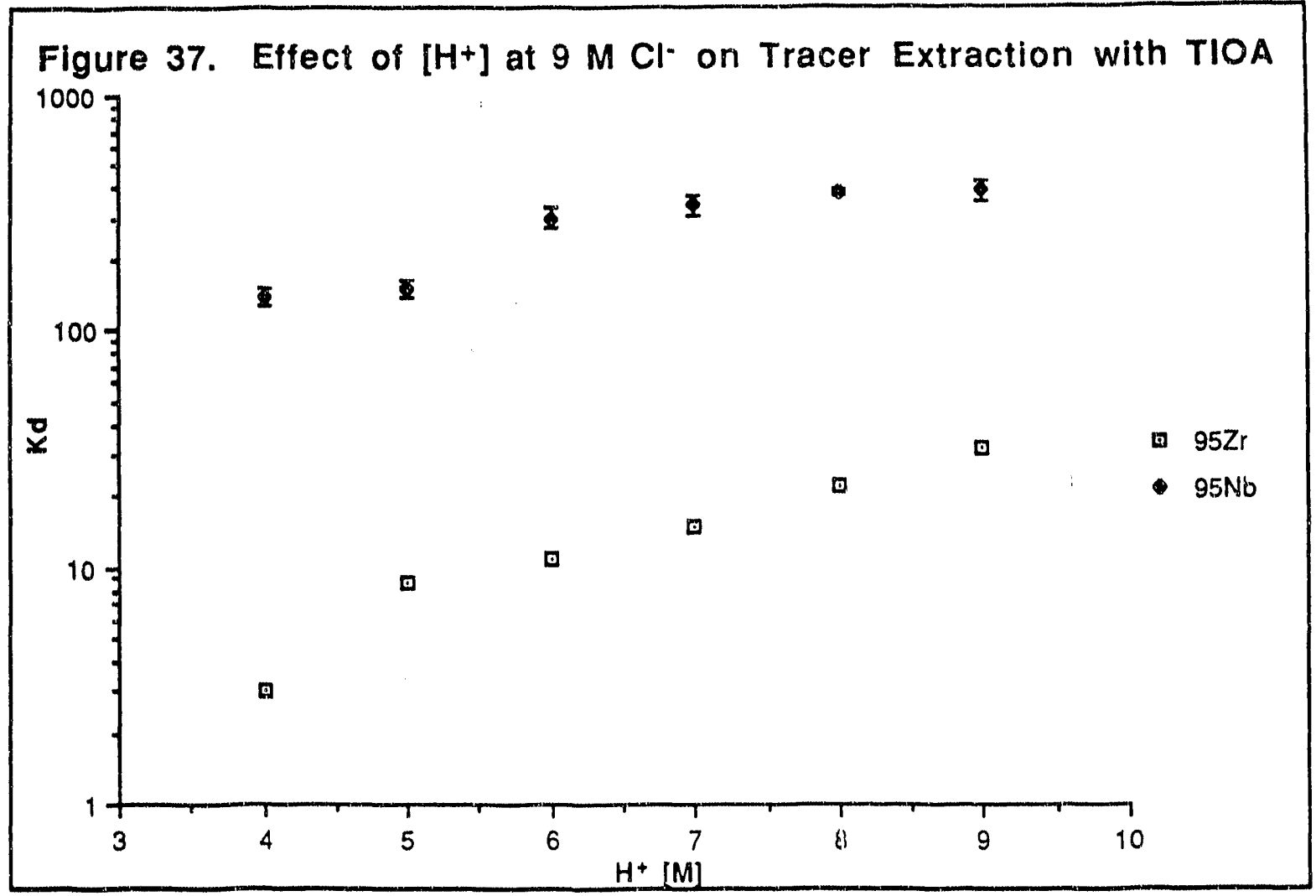

Figure 38. Effect of $\left[\mathrm{H}^{+}\right]$at $12 \mathrm{M} \mathrm{Cl}^{-}$on Tracer Extraction with TIOA

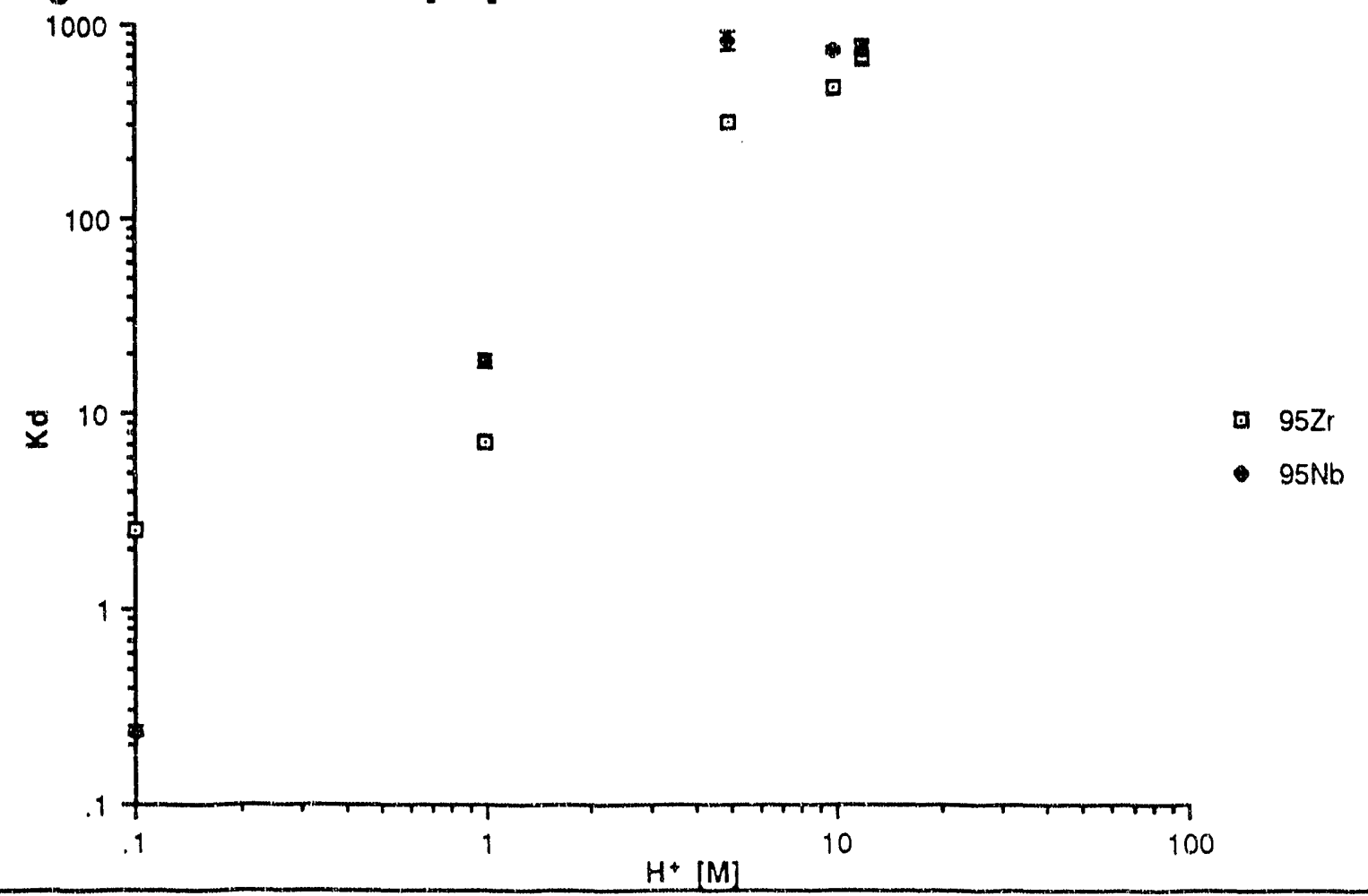


These extraction studies showed similar results to Figures 23-26. The zirconium extraction increased as a function of chloride concentration for both 1 $\mathrm{M}$ and $7 \mathrm{M}$ hydrogen ion concentration. The hydrogen ion concentration had a minimal effect on zirconium distribution coefficients (see Figure 37). The $K_{d}$ 's increased 1 order of magnitude between $4 M$ and $9 M$ hydrogen ion concentration. Over a similar range with chloride, the distribution coefficient for zirconium increased 2 to 3 orders of magnitude. However, Figure 38 shows the hydrogen ion concentration range over which metal hydrolysis and increased TIOA protonation occurs. At the low hydrogen ion concentration $(0.1 \mathrm{M})$, zirconium has a larger extraction coefficient than niobium. Being a pentavalent metal, niobium hydrolyses more easily than zirconium. The hydrolysis at low hydrogen ion concentration inhibits niobium extraction.

A final microscale experiment with TIOA was conducted (Figure 39). The experiment was designed to show that the extractions conducted with $500 \mu \mathrm{L}$ volume will work with $20 \mu \mathrm{L}$, the volume to be used for the rutherfordium extractions. The aqueous phase was $12 \mathrm{M} \mathrm{HCl}$ and the concentration of TIOA in the organic phase was varied. The results show the microscale extraction to behave the same as larger volumes.

\section{Rutherfordium Results}

Based on the previously mentioned results, rutherfordium extractions were conducted with TIOA in benzene. The aqueous phase was $12 \mathrm{M} \mathrm{HCl}$. The organic phase was $1.0 \mathrm{M}$ and $0.1 \mathrm{M}$ TIOA in benzene. At the collection site, the ${ }^{261} \mathrm{Rf}$ with the $\mathrm{KCl}$ aerosol was collected on a Teflon disk for 90 seconds. The ${ }^{261} \mathrm{Rf}$ was removed from the Teflon disk using $10 \mathrm{uL}$ of aqueous phase and was added to $20 \mu \mathrm{L}$ of organic phase in a $1 \mathrm{~mL}$ centrifuge cone and mixed in a sonicator for 5 seconds. The solution was centrifuged for 10 seconds to separate the phases. The organic phase was removed and placed on a glass 
cover slip on a hot plate evaporating the organic phase, leaving the ${ }^{261} \mathrm{Rf}$ on the cover slip. The slip was placed over a PIPS detector and counted for alpha emissions and spontaneous fissions. The presence of rutherfordium was detected from alpha decay. The results from these experiments are presented in Table 14.

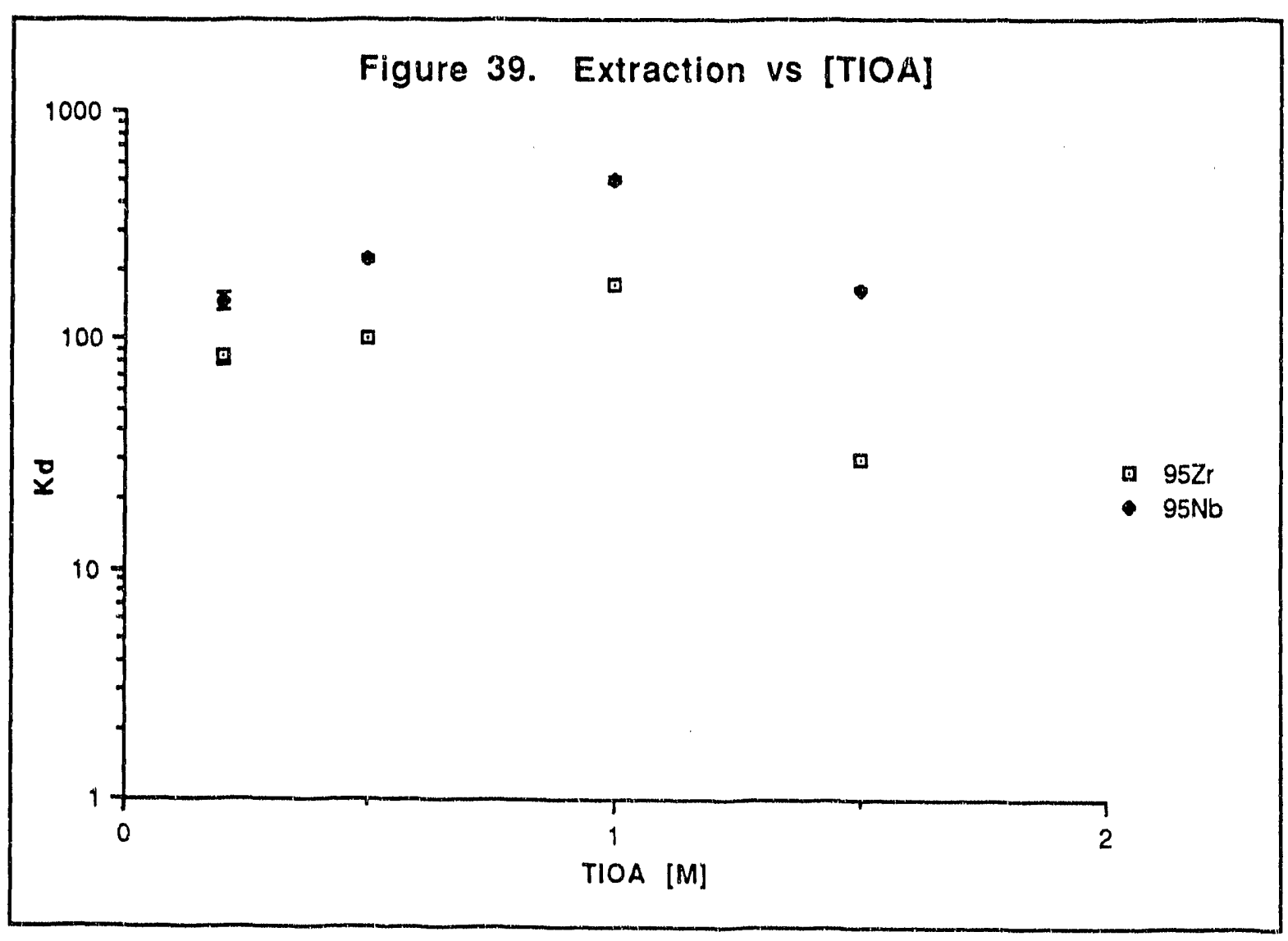

\begin{tabular}{|llll|}
\hline & \multicolumn{2}{l}{ Table 14. 261Rf TIOA Extraction Data } \\
[TIOA]M & $\begin{array}{l}\text { Extraction } \\
(\%)\end{array}$ & \# Events & \# Experiments \\
1.0 & $29.1 \pm 6.5$ & 20 & 343 \\
0.1 & $117 \pm 22.0$ & 28 & 120 \\
\hline
\end{tabular}


A total of $343^{261} \mathrm{Rf}$ extraction experiments were conducted using $1 \mathrm{M}$ TIOA in benzene. A rate of 1 event $/ 5$ experiments is expected for $100 \%$ extraction chemical yield. The region of interest for alpha decay from $261 \mathrm{Rf}$ is 8.15 to 8.35 $\mathrm{MeV}$. The experiment resulted in $20261 \mathrm{Rf}$ events, a $(29.1 \pm 6.5) \%$ chemical yield and $a K_{d} \approx 0.22$. For the $0.1 \mathrm{M} \mathrm{TIOA}$ experiments, 120 extractions were conducted. A total of 28 events were observed, giving a (117 \pm 22.0$) \%$ chemical yield. The distribution coefficient could not be calculated for the $0.1 \mathrm{M}$ TIOA concentration due to the high chemical yield. At $100 \%$ chemical yield, the amount of activity left in the aqueous phase is unmeasurable. From the equation for the calculation of the distribution coefficient, at $100 \%$ chemical yield, the $K_{d} \approx \infty$. The equation to approximate the $K_{d}$ from the percent extraction for rutherfordium is:

$$
K_{d} \approx \frac{\% \text { Extracted } / 2}{(1-\% \text { Extracted })}
$$

These rutherfordium extraction results are consistent with the zirconium tracer loss studies (Figure 34). The difference in the rutherfordium extraction at the different TIOA concentrations results from losses due to evaporation of the organic phase. Figure 39 shows that both TIOA concentrations should extract about $100 \%$ of the rutherfordium. The loss in $0.1 \mathrm{M} \mathrm{TIOA}$ is minimal over the temperature range examined. The loss for $1.0 \mathrm{M}$ TIOA increases as a function of temperature. For the rutherfordium experiments, the hot plate temperature for evaporation was $320-370^{\circ} \mathrm{C}$. A high temperature had to be maintained for rapid evaporation. Therefore, the hot plate temperature had to be at or near the boiling point of $\operatorname{TIOA}\left(365-367^{\circ} \mathrm{C}\right)$. This accounts for the observed difference in the results. 
The TIOA extraction experiment is similar to that of Hulet ef al. [HUL80]. In their experiment, the chloride complexes of hafnium, curium, fermium, and rutherfordium were tested for their relative absorption on a column containing quaternary amines held on an inert support. It was shown that rutherfordium behaved like hafnium. The major problem with those experiments was the small event rate, only 6 events. The liquid-liquid extraction studies with TIOA are more rapid, and give a higher event rate. The TIOA extraction studies showed that rutherfordium behaves differently than thorium and europium and most similarly to zirconium. Along with the results from Hulet, this result is further evidence that rutherfordium is a Group 4 element [HUL80]. 


\section{Tributyl Phosphate}

\section{Introduction}

This section describes experiments on tracer extractions using a liquid-liquid extraction system with tributyl phosphate (TBP). There have been many applications of TBP for extraction of metals. In the nuclear field, it has been extensively used in the reprocessing of spent nuclear fuel to separate and purify uranium, plutonium [MAR69], and thorium [BEC70] from fission products. The extractive powers of TBP for thorium and plutonium were noted by Manhattan Project researchers [KAT86A], [COL78]. However, only undiluted TBP was used as an extractant. Undiluted TBP has a high viscosity and a density similar to water. Furthermore, it was believed that TBP would rapidly hydrolyze in acid. For these reasons, TBP was not considered an appropriate extractant for the Manhattan Project.

Extractions utilizing dilute TBP were conducted in the late 1940's [COL78]. These results led to the development of a TBP extraction process to remove uranium from wartime reprocessing plant waste at Oak Ridge National Laboratory. The TBP extraction process was eventually used to reprocess spent nuclear fuel. The developed procedure is called the PUREX (PlutoniumUranium Befining by EXtraction) process [COL78]. Various PUREX process flow sheets were developed with 30\% TBP and implemented at Savannah River and Hanford.

TBP forms adducts with neutral metal salts. The extractability of TBP is due to the phosphoryl functional group. The phosphoryl oxygen coordinates to the metal. There is no evidence that the ether oxygens between the butyl groups and the phosphorus atom enter into metal-TBP bonding [SAI63]. The adduct formation reaction is shown in Equation 3:

$$
M^{n+}{ }_{a q}+n A^{-}{ }_{a q}+x T B P \leftrightarrow M A_{n} T B P_{x}
$$


An example of adduct formation is the extraction of uranyl nitrate as $\mathrm{UO}_{2}\left(\mathrm{NO}_{3}\right)_{2} \cdot 2 \mathrm{TBP}$. The aqueous phase may also contain charged metal-ligand species, along with hydrolyzed metal species at lower acid concentrations. However, only neutral metal complexes extract.

Experimental observations have recorded properties consistent with extracted metal-TBP species. Only neutral metal ligand species extract. The metal TBP extracted species is only slightly ionized in the organic phase. Ionization increases as a function of acid and TBP concentration. Perchloric acid forms ionized complexes with TBP considerably more than other inorganic acids [SCH84]. Multiple forms of some metal halides can be extracted, including acid species. As an example, the following zinc chloride species are extracted:

$$
\mathrm{ZnCl}_{2}, \mathrm{HZnCl}_{3}, \mathrm{H}_{2} \mathrm{ZnCl}_{4}
$$

All of these forms are neutral species [SCH84].

TBP, like other esters, is susceptible to hydrolysis in weak acids. The reaction is as follows and forms dibutylphosphoric acid (HDBP).

$$
\left(\mathrm{C}_{4} \mathrm{H}_{9} \mathrm{O}\right)_{3} \mathrm{PO}+\mathrm{HOH} \Leftrightarrow\left(\mathrm{C}_{4} \mathrm{H}_{9} \mathrm{O}\right)_{2} \mathrm{PO}(\mathrm{OH})+\mathrm{C}_{4} \mathrm{H}_{9} \mathrm{OH}
$$

Further hydrolysis leads to the formation of monobutyl phosphoric acid and eventually phosphoric acid [BLU45].

Most of the studies of TBP hydrolysis used nitric acid systems. The results show that TBP hydrolyzes faster in aqueous media than organic. Investigations have shown that TBP hydrolysis is a first order reaction with a rate of $-\mathrm{d}[\mathrm{TBP}] / \mathrm{dt}$ $=\mathrm{kTBP}$. The rate constants for TBP hydrolysis are small. At room temperature, the rate constant $\left(\mathrm{hr}^{-1}\right)$ in nitric acid is around 10.6 [SHC84A]. TBP hydrolysis in 
$\mathrm{HCl}$ solutions is similar [SHC84A]. The hydrolysis rate due to photochemical reactions is on the same order.

TBP can also extract acids. Under certain conditions, metal extraction will be reduced by competition from acid extraction. The factors affecting acid extraction include acid concentration, size and hydration of the anion [MAR69A]. The order of acid extractability for TBP is: oxalic $\approx$ acetic $>\mathrm{HClO}_{4}>\mathrm{HNO}_{3}>$ $\mathrm{HCl}>\mathrm{H}_{2} \mathrm{SO}_{4}$ [NAl62]. In HCl extraction, the complex is believed to be $2 \mathrm{TBP} \cdot \mathrm{HCl} \cdot 6 \mathrm{H}_{2} \mathrm{O}$ [KER60]. Even at high $\mathrm{HCl}$ concentration, there is little competition with metal extraction. However, at high concentration nitric acid will compete with metal extraction [MAR69A].

\section{2. $95 \mathrm{Zr}-95 \mathrm{Nb}$ Extractions}

Extraction studies of rutherfordium homologs by TBP were carried out initially with $95 \mathrm{Zr}$ tracer. The studies examined the effect of TBP, $\mathrm{HCl}$, chloride, and hydrogen ion concentrations on zirconium extraction. The TBP was purchased from Aldrich Chemical Company and used as received. The TBP physical properties are presented in Tables 15 and 16. All other chemicals used were of analytical pure grade. Benzene was used as the diluent. It was chosen because of its superior results with TIOA (see Section 3.C.3). In addition, aromatic hydrocarbons, especially benzene, are preferred diluents from research on the PUREX process. They have higher extraction coefficients for metal and do not form a third phase as readily as non-aromatic hydrocarbons [SCH84B].

In the first experiment the TBP concentration in the organic phase was varied and the aqueous phase was $12 \mathrm{M} \mathrm{HCl}$. The effect of $\mathrm{HCl}$, chloride, and hydrogen ion concentration on extraction was studied next. The organic phase was $1 \mathrm{M}$ TBP in henzene. Initially, the aqueous phase contained the radioactive tracer. The tracer stock solution was in $12 \mathrm{M} \mathrm{HCl}$ and the chloride 
concentration was varied with $\mathrm{LiCl}$. The effect of $\mathrm{HNO}_{3}$ concentration on extraction was also studied. For this experiment, the tracer was in $14 \mathrm{M} \mathrm{HNO}_{3}$ and the organic phase was $1 M T B P$ in benzene. The composition of the phases in these experiments are listed in Table 17.

All solutions were pre-equilibrated just prior to the extractions. The aqueous and organic phases were $500 \mu \mathrm{L}$ each. The phases were mixed for 2 minutes, centrifuged for 30 seconds, separated, and counted in a germanium spectrometer system as described in Section 2.C. From counts of the aqueous and organic phases, the percent extraction, or distribution coefficients ( $K_{d}=$ (cts $\mathrm{org} / \mathrm{vol} \mathrm{org}) /(\mathrm{cts}$ aqueous/vol aqueous)) can be calculated.

\begin{tabular}{|llll|}
\hline $\begin{array}{l}\text { Molecular } \\
\text { Weight }\end{array}$ & $\begin{array}{l}\text { Table } \\
\text { Boilling Point Density } \\
\left({ }^{\circ} \mathrm{C}\right)\end{array}$ & $\begin{array}{c}\text { TBP Physical Data at } 25{ }^{\circ} \mathrm{C} \\
(\mathrm{g} / \mathrm{mL})\end{array}$ & $\begin{array}{c}\text { Solubility } \\
(\mathrm{mol} / \mathrm{L})\end{array}$ \\
266.32 & $284 \pm 5$ & 0.9727 & TBP in Water Water in TBP \\
\hline
\end{tabular}

Reference [MAR69B]

\begin{tabular}{|c|c|c|c|c|c|}
\hline \multirow{2}{*}[\mathrm{HCl}]{$\mathrm{M}$} & \multicolumn{5}{|c|}{$\begin{array}{c}\text { Table 16. Solubility of TBP in } \mathrm{HCl} \text { Solutions } \\
\text { Solubility (mM) }\end{array}$} \\
\hline & $5^{\circ} \mathrm{C}$ & $15^{\circ} \mathrm{C}$ & $25^{\circ} \mathrm{C}$ & $35^{\circ} \mathrm{C}$ & $45^{\circ} \mathrm{C}$ \\
\hline 0 & 3.6 & 2.4 & 1.68 & 1.3 & 1.1 \\
\hline 1 & 3.2 & 2.2 & 1.67 & 1.3 & 1.2 \\
\hline 2 & 2.7 & 2.0 & 1.66 & 1.4 & 1.3 \\
\hline 3 & 2.0 & 1.9 & 1.68 & 1.5 & 1.5 \\
\hline 4 & 2.4 & 2.0 & 1.88 & 1.8 & 1.7 \\
\hline 6 & 2.5 & 2.2 & 2.17 & 2.2 & 2.1 \\
\hline 7 & 2.9 & 2.9 & 2.93 & 2. & 2.9 \\
\hline 8 & $\cdot$ & $\cdot$ & 3.26 & - & - \\
\hline 9 & $\cdot$ & - & 3.56 & - & - \\
\hline
\end{tabular}




\begin{tabular}{|llll|}
\hline \multicolumn{4}{c}{ Table 17 TBF Variable Concentrations } \\
$\begin{array}{l}\text { Component } \\
\text { Varled }\end{array}$ & Aqueous Phase & $\begin{array}{c}\text { Organic Phase } \\
\text { [TBP] M }\end{array}$ & Figure \# \\
TBP & $12 \mathrm{M} \mathrm{HCl}$ & $0.25,0.5,1,0,1.5,2.0$ & 40 \\
$\mathrm{HCl}$ & $2,4,6,8,10,12 \mathrm{M} \mathrm{HCl}$ & 1.0 & 41 \\
$\mathrm{Cl}^{-}$ & $6 \mathrm{M} \mathrm{H}^{+} ; 6,8,10 \mathrm{M} \mathrm{Cl}^{-}$ & 1.0 & 42 \\
$\mathrm{H}^{+}$ & $6 \mathrm{M} \mathrm{Cl}^{-} ; 2,4,6 \mathrm{M} \mathrm{H}^{+}$ & 1.0 & 43 \\
$\mathrm{HNO}_{3}$ & $4,6,8,10,12,14 \mathrm{MHNO}_{3} 1.0$ & 44 \\
\hline
\end{tabular}

\section{3. $95 \mathrm{Zr}-95 \mathrm{Nb}$ Extraction Results and Conclusions}

In the first experiment the effect of TBP concentration on tracer extraction was examined (Figure 40). The results show a maximum in the zirconium extraction at 1 M TBP where the maximum extraction was at $K_{d}=330$. This result is sirnilar to the TIOA extraction of zirconium where maximum extraction also occurs at $1 \mathrm{M}$ TIOA (Figures 20 and 31). Above $1 \mathrm{M}$ concentration of ligand, aggregation occurs and inhibits zirconium extraction, as mentioned in Section 3.C.1. The extraction curve for niobium differs from the zirconium extraction curve. The extraction of niobium reaches a plateau at 1 M TBP. Within the error limits of the data, niobium extraction remains constant as the TBP concentration is increased beyond $1 \mathrm{M}$.

The next experiment measured the effect of $\mathrm{HCl}$ concentration on zirconium extraction (Figure 41). The results show that extraction of zirconium and niobium both increase as a function of $\mathrm{HCl}$ concentration. At low concentrations, both metais extract poorly. The niobium extraction increases immediately as the $\mathrm{HCl}$ concentration is increased, while the zirconium extraction begins to increase between 4 and $6 \mathrm{M} \mathrm{HCl}$. Both tracers show little increase in extraction above $10 \mathrm{M} \mathrm{HCl}$ within the measurable limit of the 
experiment. Generally, niobium has a larger distribution coefficient than zirconium.

The effect of chloride concentration on zirconium extraction was also studied (Figure 42). The hydrogen ion concentration was kept constant at $6 \mathrm{M}$, and the chloride concentration was varied from 6 to $10 \mathrm{M}$ with $\mathrm{LiCl}$. The results show that the extraction of both tracers increases with increasing chloride concentration. Again, the niobium extraction is greater than the zirconium. The niobium extraction begins to level off at $8 \mathrm{M}$ chloride, while the zirconium extraction continually increases up to $10 \mathrm{M}$ chloride.

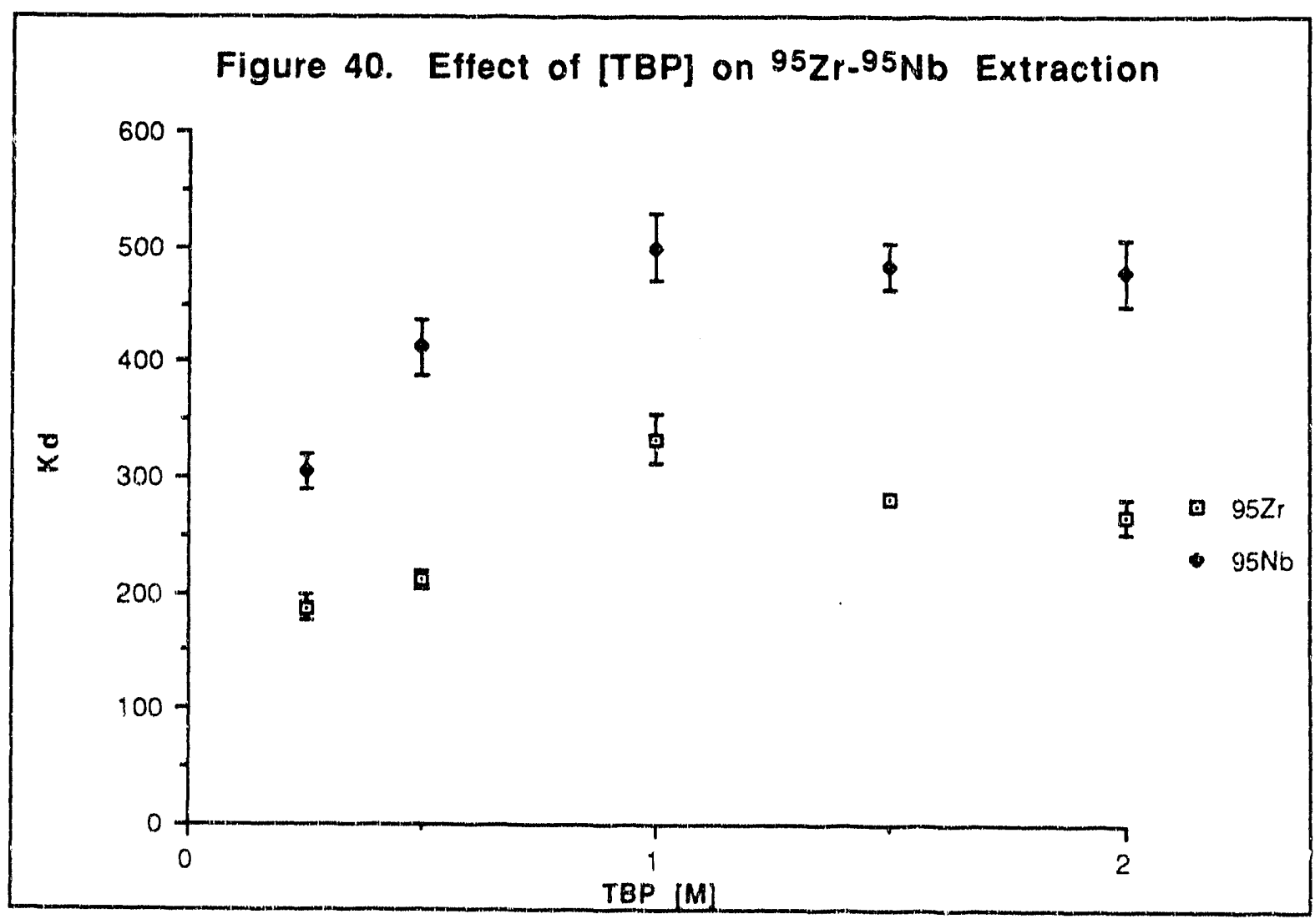



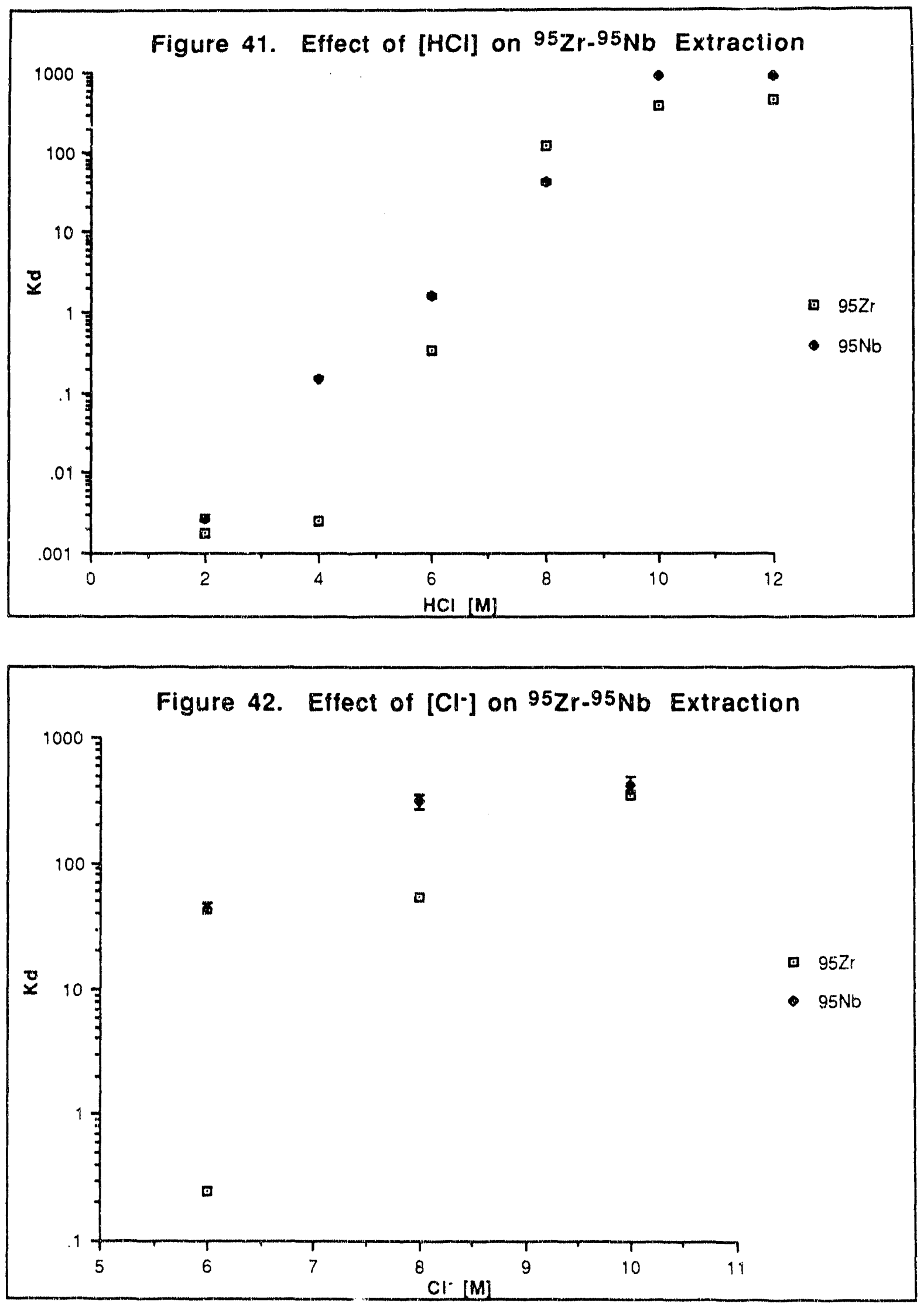
The effect of hydrogen ion concentration on zirconium extraction was also studied (Figure 43). Hydrogen ion concentration has a greater effect on zirconium than niobium extraction. Over the range examined, 2 to $6 \mathrm{M} \mathrm{HCl}$, the zirconium distribution coefficient increases by about 2 orders of magnitude while the niobium distribution varied by less than an order of magnitude. As with the other systems, niobium extraction is greater than zirconium.

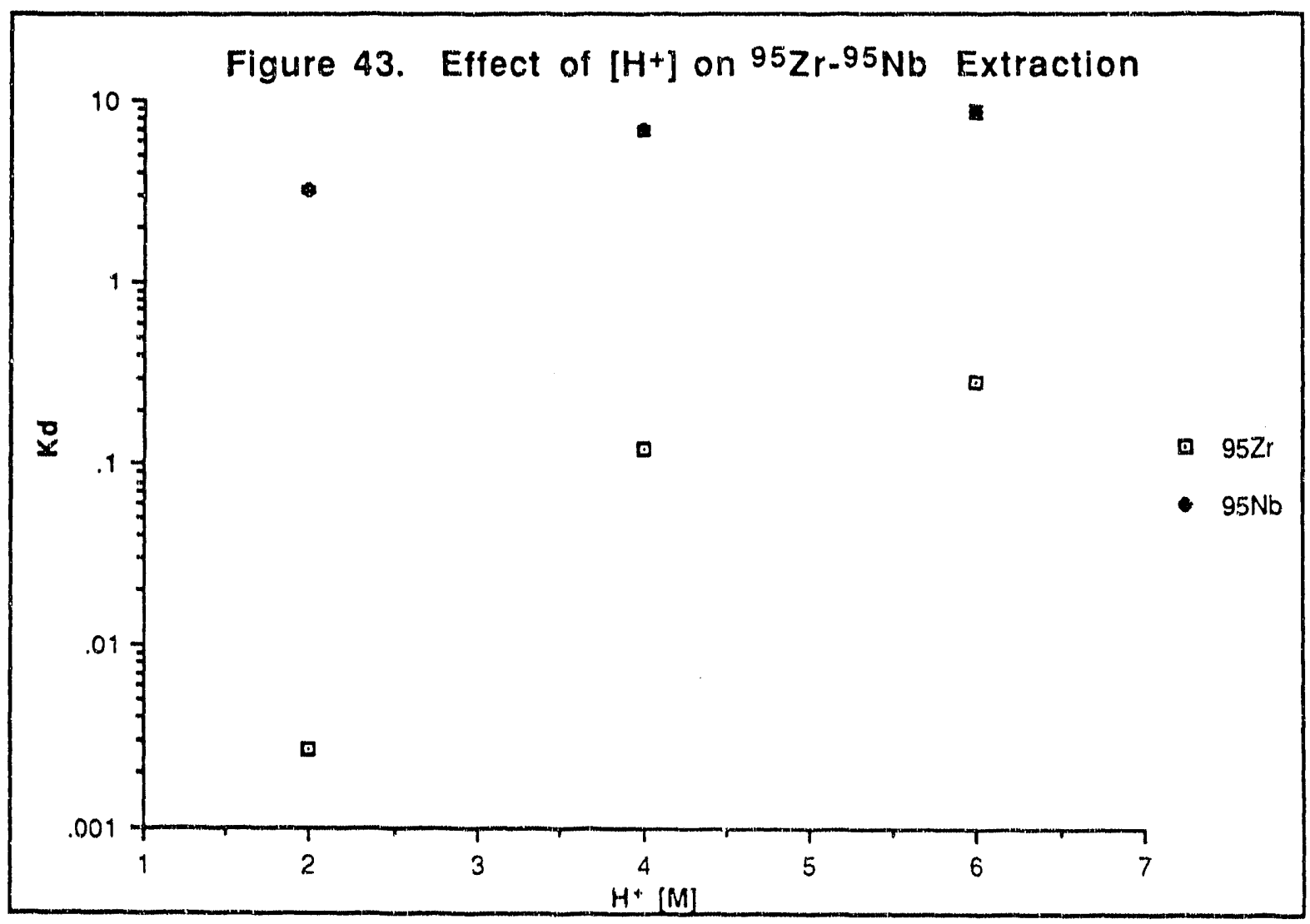

A final experiment was conducted on the effect of nitric acid concentration on zirconium extraction (Figure 44). In this experiment, the organic phase was $1 \mathrm{M}$ TBP in benzene and the concentration of nitric acid in the aqueous phase was varied. The experiment showed that zirconium extracts better than niobium. However, the zirconium extraction decreases when the nitric acid concentration exceeds $12 \mathrm{M}$. This is due to TBP extraction of nitric acid. At this concentration, 
nitric acid can successfully compete with zirconium for TBP extraction. The results of this experiment gave further support for the use of $\mathrm{HCl}$ as the aqueous phase. Unlike nitric acid, $\mathrm{HCl}$ does not successfully compete with zirconium for TBP (Figure 41).

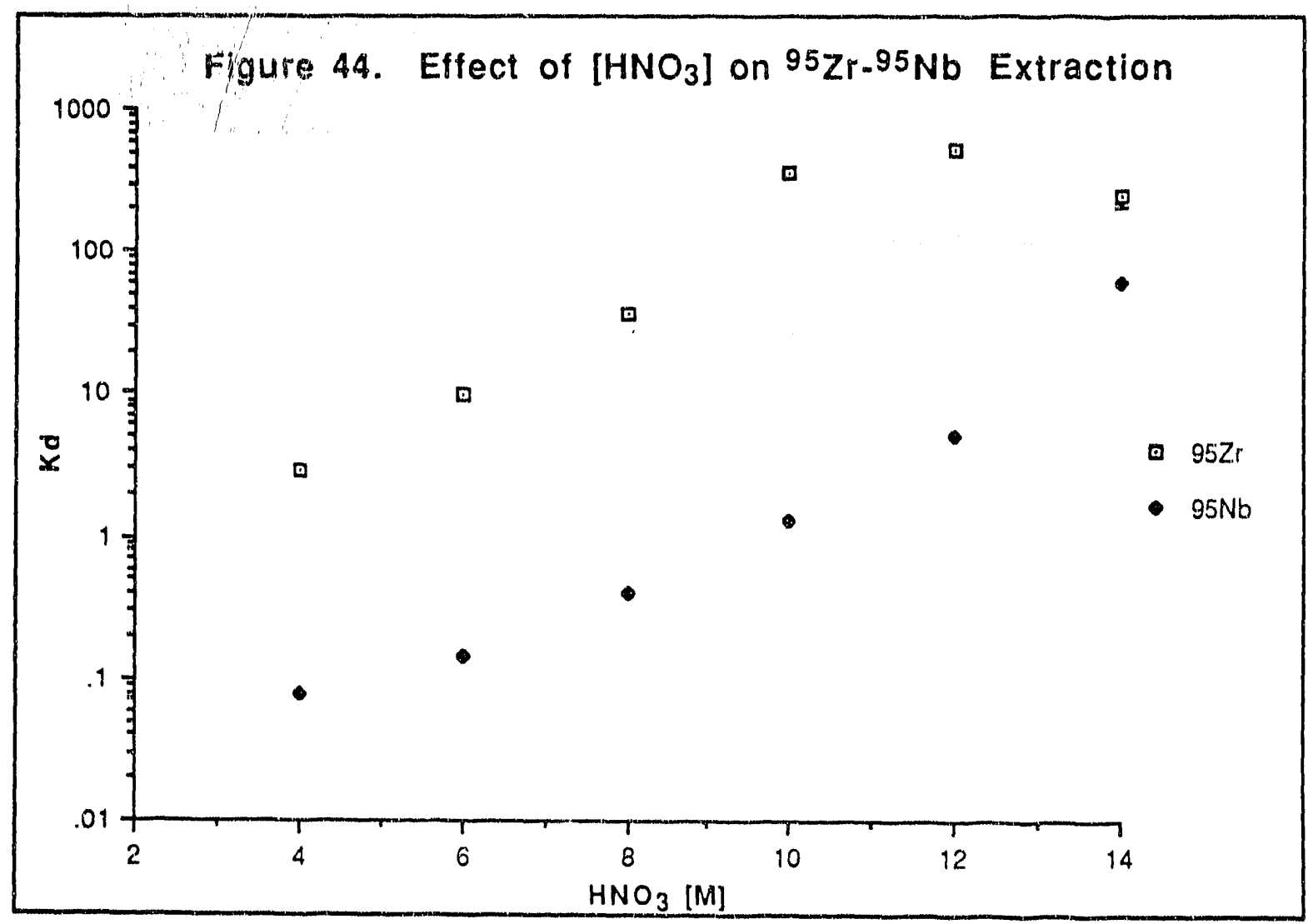

The results from these series of experiments were used to determine which type of organic and aqueous phases would be preferred for rutherfordium extraction studies. Zirconium extractions were high $\left(\mathrm{K}_{d}>10\right)$ for $\mathrm{HCl}$ and Chloride concentrations above $8 \mathrm{M}$. Therefore, it was decided to examine rutherfordium extraction with TBP at $\mathrm{HCl}$, chloride, and hydrogen ion concentrations between 8 and $12 M$. A concentration of 1 M TBP in benzene was used for the tracer experiments. However, 0.25 M TBP benzene was used for rutherfordium extractions. The lower TBP concentration was easier to 
evaporate and facilitated thin sample preparation. The lower TBP concentration should not greatly decrease the extent of rutherfordium extraction. From Figure 40 , the zirconium distribution coefficient is 175 for $0.1 \mathrm{M}$ TBP. This is lower than the maximum distribution coefficient of 330 ai $1.0 \mathrm{M}$ TBP. However, both these distribution coefficients represent over $99 \%$ extraction.

\section{Rutherfordium and Homolog Extraction Results}

Tracer studies of the extraction of rutherfordium homologs were carried out using ${ }^{95} \mathrm{Zr}$, ${ }^{169} \mathrm{Hf},{ }^{228} \mathrm{Th}$, and ${ }^{238} \mathrm{Pu}$. The ${ }^{95} \mathrm{Zr},{ }^{228} \mathrm{Th}$, and ${ }^{238} \mathrm{Pu}$ were used in off-line experiments while $169 \mathrm{Hf}$ was produced on-line at the 88 -inch Cyclotron using a $\mathrm{Gd}_{2} \mathrm{O}_{3}$ target with ${ }^{18} \mathrm{O}$ projectiles as described in Section 2.A.4.c.

All solutions were pre-equilibrated prior to the experiments. The experiments using ${ }^{95} \mathrm{Zr}$ and ${ }^{228} \mathrm{Th}$ tracers were conducted with $500 \mu \mathrm{L}$ each of the aqueous and organic phases. In the ${ }^{169} \mathrm{Hf}$ experiments, $10 \mu \mathrm{L}$ of aqueous phase and $20 \mu \mathrm{L}$ of organic phase were used. In the $238 \mathrm{Pu}$ experiments $100 \mu \mathrm{L}$ of each phase were used.

The aqueous phase contained various $\mathrm{HCl}$, chloride or hydrogen ion concentrations and the radioactive tracer. The $\mathrm{HCl}$ concentration was varied between $8 \mathrm{M}$ and $12 \mathrm{M}$, the chloride concentration was adjusted to $8 \mathrm{M}$ and 12 $\mathrm{M}$ using $\mathrm{LiCl}$ with $\left[\mathrm{H}^{+}\right]=8 \mathrm{M}$, and the hydrogen ion concentration was varied between $8 \mathrm{M}$ and $12 \mathrm{M}$ with the chloride concentration held constant at $12 \mathrm{M}$. The tracer stock solutions were $12 \mathrm{M} \mathrm{HCl}$ with a specific activity of $20 \mu \mathrm{Ci} / \mathrm{mL}$. The organic phase consisted of 1 M TBP in benzene for experiments with 238 Pu extraction for varied $\mathrm{HCl}$ concentrations and $0.25 \mathrm{M}$ TBP in benzene for all other extraction studies.

For the experiments with ${ }^{95} \mathrm{Zr},{ }^{228} \mathrm{Th}$, and ${ }^{238} \mathrm{Pu}$, the aqueous and organic phases were vigorously mixed for 2 minutes, and centrifuged for 30 seconds to separate the phases. The phases were separated and assayed for alpha and 
gamma activity as described in Section 2.C. For the $169 \mathrm{Hff}$ experiments, the phases were mixed using a sonic mixer for 5 seconds and separated by centrifuging for 10 seconds. The phases were separated and assayed for gamma activity. From counts of the aqueous and the organic phases the percent extraction or distribution coefficients were calculated.

The rutherfordium experimental conditions were similar to those of $169 \mathrm{Hf}$. At the collection site, the ${ }^{261} \mathrm{Rf}$ with an $\mathrm{KCl}$ aerosol was collected on a Teflon disk for 90 seconds. The deposited ${ }^{261} \mathrm{Rf}$ was removed from the Teflon disk with 10 $\mu \mathrm{L}$ of aqueous phase and added to $20 \mu \mathrm{L}$ of the organic phase in a $1 \mathrm{~mL}$ centrifuge cone and mixed in a sonicator for 5 seconds. The solution was centrifuged for 10 seconds to separate the phases. The organic phase was removed and placed on a glass cover slip on a hot plate to evaporate the organic phase, leaving the ${ }^{261}$ Rf on the cover slip. The slip was placed over a PIPS detector and counted for alpha emissions and spontaneous fissions. The presence of rutherfordium was detected from alpha decay.

The effect of $\mathrm{HCl}$ concentration on extraction is shown in Figure 45. The organic phase was 1 M TBP in benzene for plutonium. In all other experiments the organic phase was $0.25 \mathrm{M} \mathrm{TBP}$ in benzene. Zirconium and plutonium extraction remained high in the $\mathrm{HCl}$ range of 8 to $12 \mathrm{M}$. The zirconium result is consistent with Figure 41 where the extraction is near $100 \%$ in the same $\mathrm{HCl}$ concentration range. The tracer results are consistent with literature data [PEP56], [CLE70]. The results show that extraction for zirconium, hafnium, thorium, plutonium and rutherfordium increases or remains high as a function of $\mathrm{HCl}$ concentration.

In the next experiments the effect of chloride concentration on extraction of rutherfordium and its homolog with the hydrogen ion held constant at $8 \mathrm{M}$ was 
examined (Figure 46). All experiments were conducted with an organic phase of $0.25 \mathrm{M}$ TBP in benzene.

Figure 45. Effect of [HCl] on Extraction of $95 \mathrm{Zr},{ }^{169} \mathrm{Hf}, 228 \mathrm{Th}$, $238 \mathrm{Pu}$, and $261 \mathrm{Rf}$

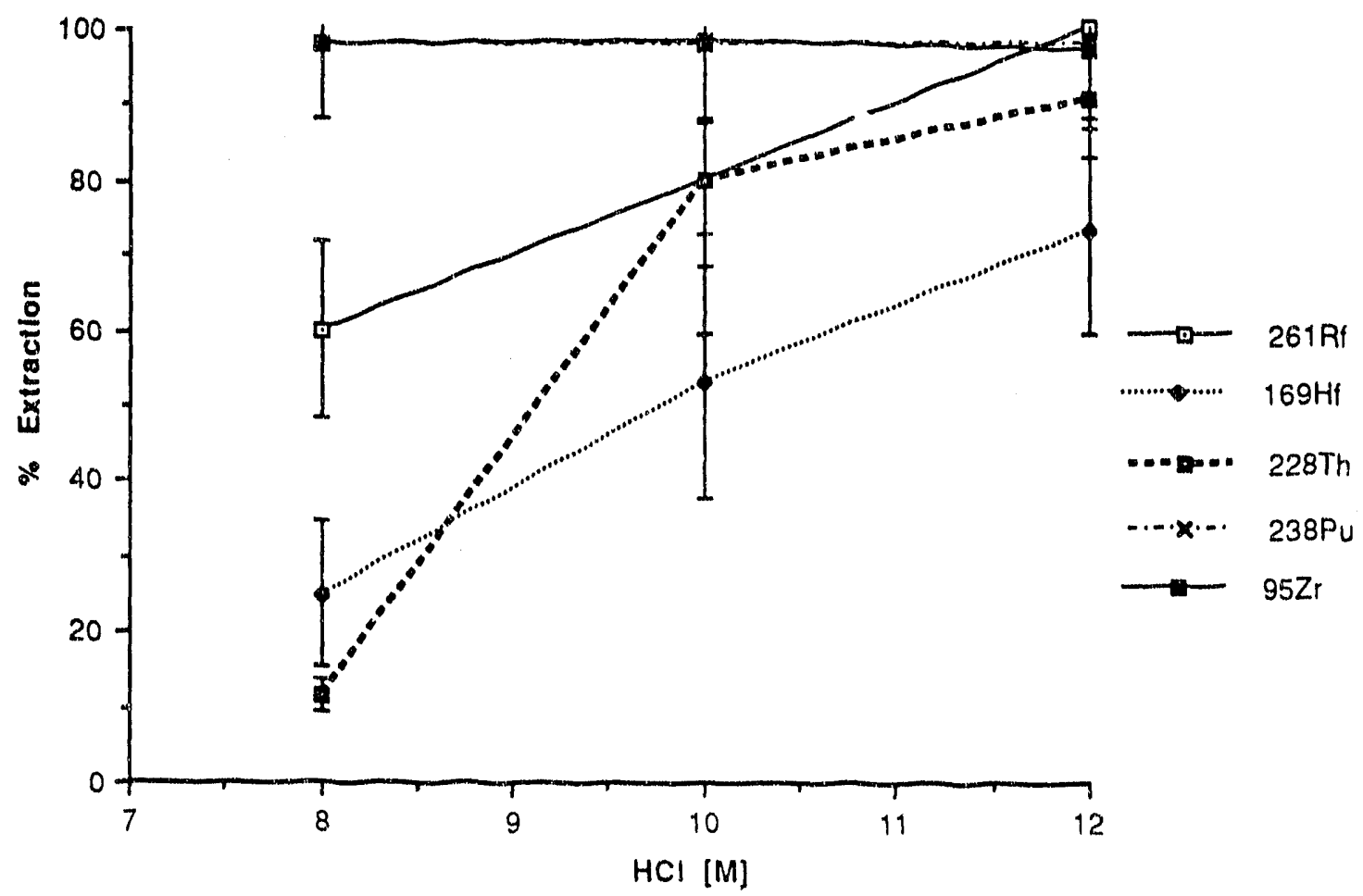

The rutherfordium extraction reached a maximum around $10 \mathrm{M}$ chloride, then decreased as the chloride concentration was increased to $12 \mathrm{M}$. The extraction for zirconium, hafnium and thorium continued to increase as a function of chloride concentration, similar to the effect with varied $\mathrm{HCl}$ concentration. The extraction of plutonium decreased as a function of chloride concentration. These results indicate that rutherfordium and plutonium form anionic chloride specie: at higher chloride concentration. These anionic species do not extract with TBP. The plutonium result is consistent with 
literature data on nitric acid systems in which plutonium forms unexiractable anionic species [SCH84].

Zirconium and hafnium form anionic chloride species at high chloride concentrations [COT72]. However, these complexes are not as strongly anionic as plutonium complexes. The TBP appears to stabilize the zirconium and hafnium neutral complexes, while it is unable to stabilize a neutra! plutonium complex at chloride concentrations above $8 \mathrm{M}$. Thorium does not form anionic chloride complexes. This experiment shows where rutherfordium deviates from the expected chemical trend of the Group 4 elements. If rutherfordium acted as the other Group 4 elements, the extraction should have increased as the chloride concentration increased. At $12 \mathrm{M}$ chloride, rutherfordium extraction was at a minimum in the experiment range while zirconium and hafnium extraction was at a maximum. The results are similar to experiments conducted on hahnium (Element 105) [GOB91], [GRE91], [KRA89]. In these experiments, hahnium is shown to act more similarly to protactinium than niobium or tantalum. 


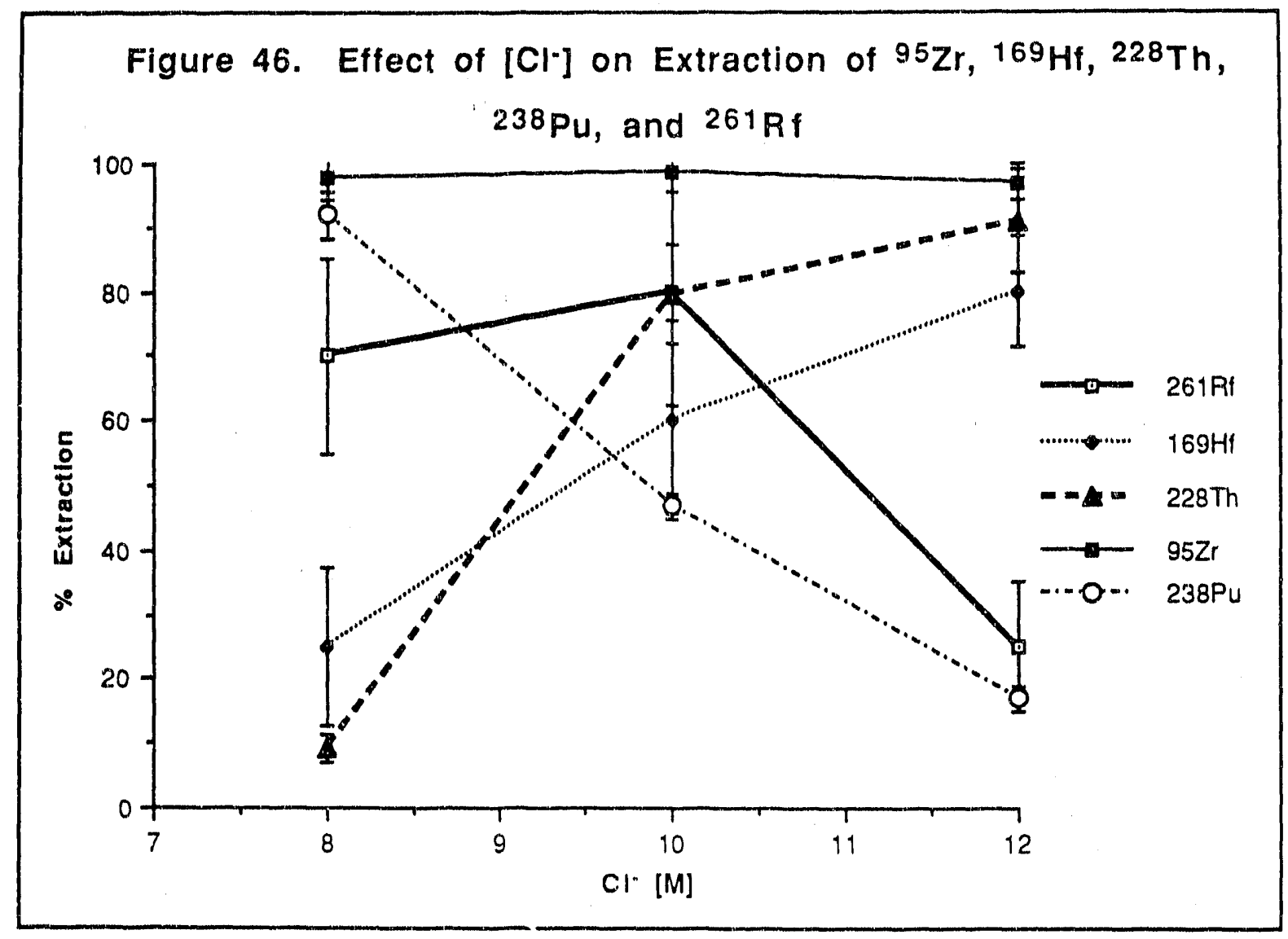

Figure 47 shows the effect of hydrogen ion concentration on rutherfordium, zirconium and hafnium extraction. Hydrogen ion concentration does not affect the extraction of zirconium and hafnium at the experimental conditions. The extraction for these elements is similar to that for $12 \mathrm{M}$ chloride and $12 \mathrm{M} \mathrm{HCl}$. On the other hand, rutherfordium extraction increases as a function of hydrogen ion concentration in the examined range. This result is consistent with the formation of a $\mathrm{HCl}$ salt as the extracted metal-ligand moiety. The extracted complex may be $\mathrm{RfCl}_{x} \mathrm{H}_{x-4}$ yTBP where $x$ is 5 or 6 . Other examples of this type of extraction with TBP have been observed earlier [SCH84]. 


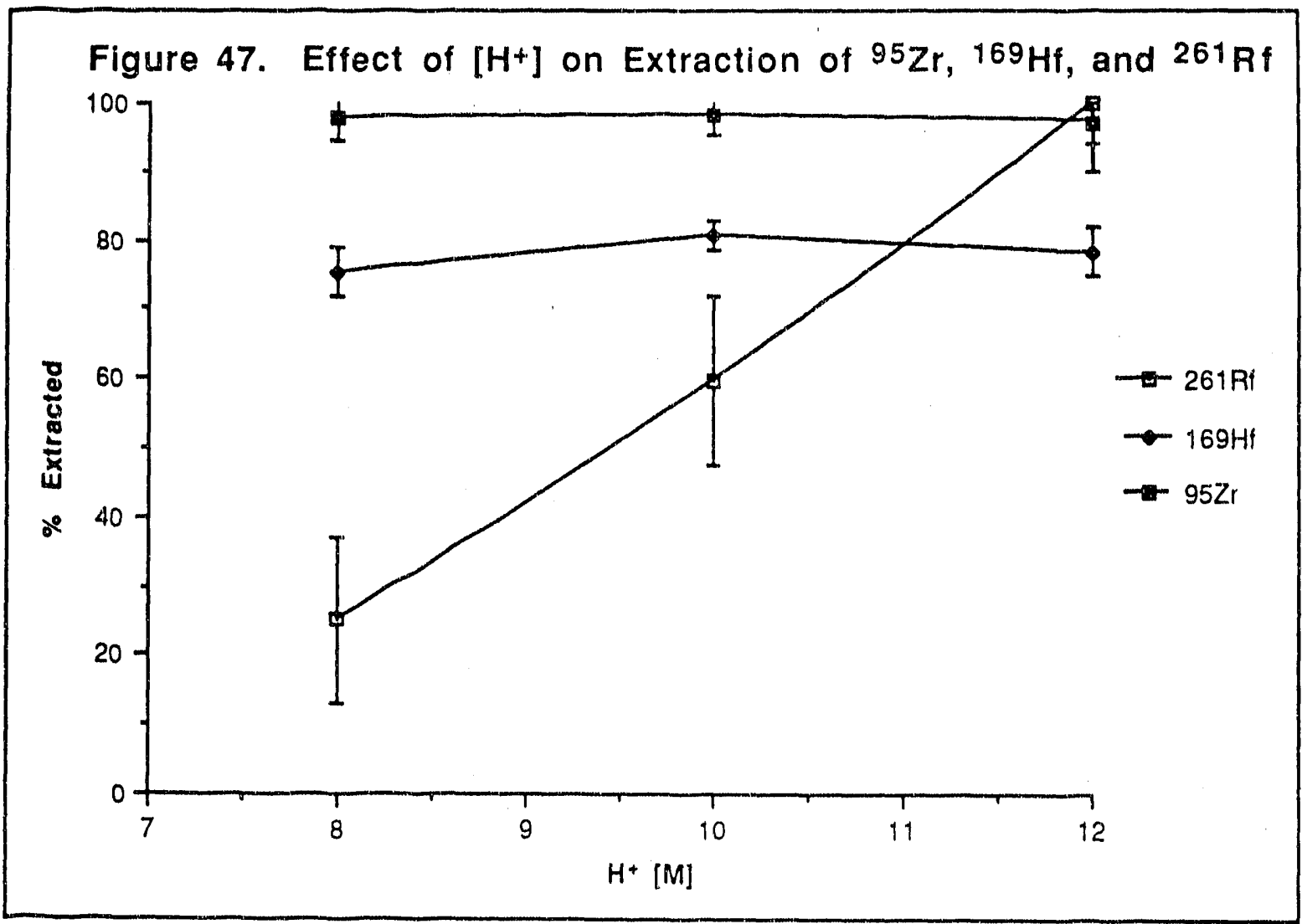

These extraction results for the first time showed evidence for a deviation of rutherfordium chemistry from the other Group 4 elements. The unexpected chloride results indicate that rutherfordium at times behaves more like tetravalent plutonium than zirconium or hafnium. In addition, rutherfordium extraction was effected by hydrogen ion concentration, while zirconium and hafnium showed no effect. These results indicate that rutherfordium forms anionic complexes more readily than zirconium or hafnium. In addition, the extracted complex for rutherfordium appears different from those of zirconium or hafnium. This is dramatically demonstrated by the difference in the effect of hydrogen ion concentration on metal extraction. These differences, along with the differences between hahnium and its Group 5 homologs, indicate an unexpected trend in the transactinide elements. 


\section{E. Thenoyltrifluoroacetone}

1. Introduction

Experiments on metal extraction utilizing thenoyltrifluoroacetone (TTA) are discussed in this section. Information on the properties of TTA are presented in Table 18. Unlike TIOA or TBP, TTA chelates the metals it extracts. This chelation forms an organic soluble complex with the metal. Generally, organic chelating agents like TTA form stable, monomeric compounds with metals. This is because the organic chelating agent acts as a weak acid, exchanging a proton for a cationic metal. The donor group complex is a bidentate chelate with TTA. If enough material is present, the complex can be isolated as a solid. Diluent effects are minimized with these compounds [MAR69C]. The general extraction equation is:

$$
x H T T A+M^{x+} \leftrightarrow M(T T A) x+x H^{+}
$$

where $M^{x+}$ is the metal or cationic metal complex.

Table 18. TTA Data

$\begin{array}{llll}\begin{array}{l}\text { Molecular } \\ \text { Formula }\end{array} & \begin{array}{l}\text { Molecular } \\ \text { Weight }\end{array} & \begin{array}{l}\text { Melting Point } \\ \left({ }^{\circ} \mathrm{C}\right)\end{array} & \begin{array}{l}\text { Boiling Point } \\ \left({ }^{\circ} \mathrm{C}\right)\end{array} \\ \mathrm{C}_{8} \mathrm{H}_{5} \mathrm{O}_{2} \mathrm{~F}_{3} \mathrm{~S} & 222.2 & 42.5-43.2 & 123\end{array}$

TTA is a popular chelating species used in the extraction of $4^{+}$metals and much work has been done with it. Studies of optimum conditions for zirconium extraction using TTA with $\mathrm{HCl}$ and $\mathrm{HNO}_{3}$ shows that $98 \%$ extraction occurs in $0.25 \mathrm{M}$ acid [ERT73]. TTA has also been used to separate zirconium from 
hafnium with an aqueous pharse of $2 \mathrm{M}$ perchloric acid [CON49]. Others used TTA to find the equilibrium constant and the heat of extraction of $\mathrm{Np}$ (IV) and Pu(IV) complexes [RAM78]. TTA will not extract $3^{+}$species under the same conditions as for $4+$ ions. For this reason, it has been used as a method of separating some $4^{+}$actinides from lanthanides [SAM80]. Investigations of tetravalent plutonium complexation by TTA from sulphate, nitrate, chloride, and fluoride aqueous solutions have been done [BAG76]. The thermodynamic properties of the extraction of thorium were determined by performing extractions at different temperatures [PAT80].

Most of the work with TTA has been done with nitric or perchloric acid in the aqueous phase. The work presented in this section was done with $\mathrm{HCl}$. Aqueous solutions of $\mathrm{HCl}$ generally result in lower $\mathrm{K}_{\mathrm{d}}$ 's for tetravalent metal extraction at the same $\mathrm{pH}$ compared to $\mathrm{HNO}_{3}$ or $\mathrm{HClO}_{4}$ [BAG76], [ERT73], [SEK67].

TTA has also been used in mixtures with other reagents in synergistic extractions, which sometimes improve results. Synergism is when two extractants have greater extracting power than the sum of the extractants individually. Studies on the adduct formation of $E u(I I I)$ and $T h(I V)$ chelate complexes of TTA and isopropyltropolone with TBP and methylisobutylketone have been conducted [SEK67]. The addition of tri-n-octyl phosphine oxide (TOPO) to TTA was found to enormously enhance the extraction of $\mathrm{Np}$ (IV) and Pu(IV) [PAT80]. However, in other instances, no positive effect was noticed. A mixture of TTA and dipentyl sulphoxide showed no strong synergistic effects in the extraction of $\mathrm{Zr}(\mathrm{IV})$ from HCI [PAN86].

The metal species involved in the extraciion with ПTA are at times not well known. The literature results differ on the form of the metal chelate species in the organic and aqueous phase. Early work was conducted in aqueous $\mathrm{HClO}_{4}$ 
solutions. In these experiments the form of the metal chelate was determined to be $\mathrm{Zr}(\pi \mathrm{TA})_{4}$ and the form of the metal in the aqueous phase was between $Z \mathrm{r}^{4+}$ and $\mathrm{Zr}(\mathrm{OH})^{3+}[\mathrm{CON} 49]$. However, others report that it is the zirconyl ion $\left(\mathrm{ZrO}^{2+}\right)$ which is in solution [ERT73], [HUF49]. Still other work claims $\mathrm{ZrO}^{2+}$ does not exist [MAC73], but, it has also been stated that $\mathrm{Zr}^{4}+$ does not exist uncomplexed to oxygen in acidic environments [COT72]. Still others believe that in slightly acidic solutions, zirconium is in the form $\mathrm{Zr}(\mathrm{OH})^{3+}$ or $\mathrm{Zr}(\mathrm{OH})_{2}{ }^{2+}$ [SOL73].

TTA was first synthesized by Reid and Calvin [REI50]. It has been one of the most wiciely used chelating agents in the solvent extraction of metal ions because of its special ability to extract metals from highly acidic solutions. Since it can operate at lower pH compared to other chelating agents, there can be better extraction without interference from hydrolysis. The extraction also has a high power dependence on acidity, making separations easily achievable through control of the pH [POS61].

The structure of TTA has the following three forms shown in Figure 48 . Of the three forms, only the enol and hydrate are important for extraction. The keto is not prevalent in either the aqueous or organic phase. In benzene, $89 \%$ of the TTA is in the enol form and $11 \%$ is in the hydrate form [KIN51]. In dilute acid, $98.4 \%$ of the TTA is in the hydrate form and $1.6 \%$ is in the enol form [REI50]. TTA is only slightly soluble in an acidic aqueous phase. The distribution coefficient of TTA between benzene and $2 \mathrm{M}$ perchiloric acid is 40 [CON49].

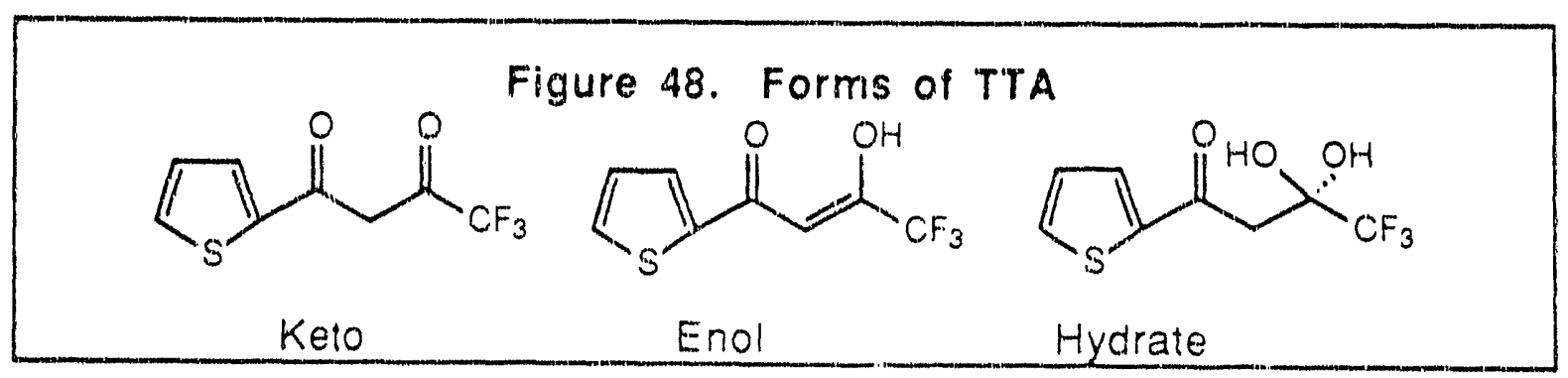


The metal is extracted from the acid to the benzene due to chelate formation involving the enolate ion and the charged metal. The $p K_{a}$ of the enolate is 6.38 [C0052]. The structure of the metal-chelate complex is shown in Figure 49. Each uncomplexed tetravalent metal can complex with up to 4 molecules of TTA. Such a complex would have 8-coordinate geometry. The chelate gains extra stability through resonance of the double bonds. This resonance is depicted in Figure 50. The neutral complex formed is soluble in the organic phase.

\section{Figure 49. Metal TTA Complex}<smiles>O=C(C=C1C=C(C(F)(F)F)ONO1)c1cccs1</smiles>

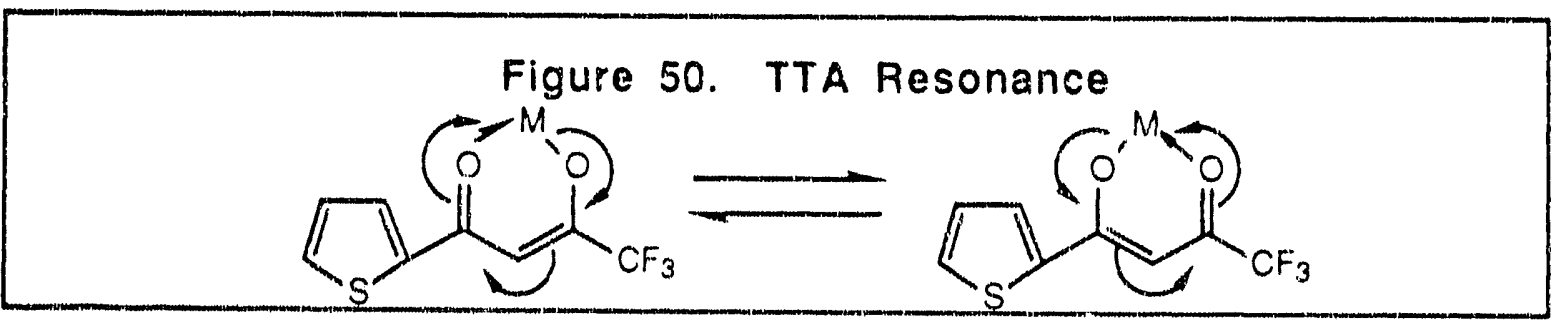

A desirable property of TTA extractions is the effect of the aqueous phase on metal selectivity. Since TTA is a weak acid, the formation of extractable complexes is dependent upon $\mathrm{pH}$. The simplified equilibrium expression for the distribution coefficient is:

$$
K_{d}=\frac{[M(T T A) \times] \text { org }}{\left(M^{+x}\right] a q}
$$


Substituting the distribution coefficient into the equilibrium constant for Equation 5 gives:

$$
K_{e q}=\frac{K_{d}\left[H^{+}\right]^{x}}{[H T T A]^{x}}
$$

Thus rearrangement of Equation 5 shows the effect of $\mathrm{pH}$ on the distribution coefficient. An adjustment of $\mathrm{pH}$ will have a profound effect on the extraction of a metal. However, this is true only up to a point. As the pH is increased, the enolate ion becomes dominant, increasing the solubility in the aqueous phase. Near $\mathrm{pH}=8, T T A$ is equally soluble in both phases. Above $\mathrm{pH}=9$, TTA cleaves into trifluoroacetic acid and acetylthiophere [POS61]. At this point, TTA no longer extracts metals.

\section{2. $95 \mathrm{Zr}$ and $162 \mathrm{Hf}$ Extractions}

All chemicals were of analytical grade. They were purchased from Aldrich Chemical Company and used as received. For the zirconium experiments, a stock solution of $95 \mathrm{Zr}$ in hydrochloric acid was prepared at a concertration of approximately $10 \mu \mathrm{Ci} / 50 \mathrm{\mu L}$. Two zirconium extraction experiments were performed. In the first experiment, the $\mathrm{HCl}$ concentration was kept at $0.24 \mathrm{M}$ while the TTA concentration was varied between $0.005 \mathrm{M}$ and $0.5 \mathrm{M}$ (Figure 51). In the second, the TTA concentration was kept at $0.25 \mathrm{M}$ while the $\mathrm{HCl}$ concentration was varied between $0.25 \mathrm{M}$ and $4 \mathrm{M}$ (Figure 52). After the solutions were prepared and pre-equilibrated, $500 \mu \mathrm{L}$ of the TTA in benzene and $500 \mu \mathrm{L}$ of the $\mathrm{HCl}$ were mixed on a vortex mixer for two minutes. Initially, the tracer was in the aqueous phase. The phases were separated by centrifuging and then transferred to vials. The gamma activity in each phase was assayed as described in Section 2.C. 

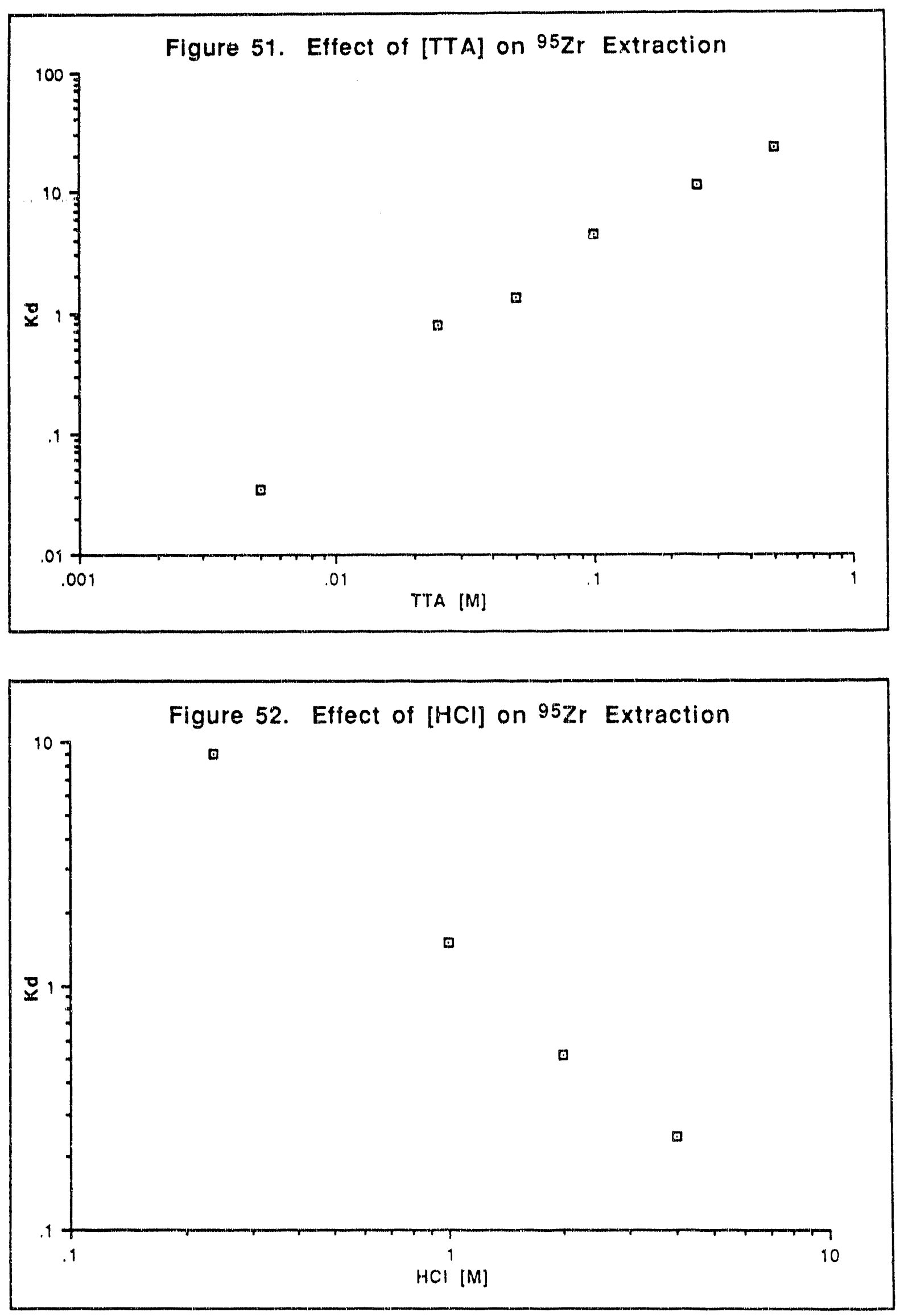
The hafnium experiments were performed at the Lawrence Berkeley Laboratory 88 -Inch Cyclotron. The isotope ${ }^{162} \mathrm{Hf}$ was produced by the ${ }^{147} \mathrm{Sm}$ $(20 \mathrm{Ne}, 5 \mathrm{n}$ ) reaction (see Section 2.A.4.b). A helium jet was used to transport the activity from the target to the lab area where the activity was deposited on Teflon squares. Experiments on ${ }^{162} \mathrm{Hf}$ used $10 \mu \mathrm{L}$ of aqueous phase and $20 \mu \mathrm{L}$ of organic phase, employing the separation procedure described for rutherfordium separations. Two extraction experiments were performed. In the first, the $\mathrm{HCl}$ concentration was kept at $0.24 \mathrm{M}$ while the TTA concentration was varied between $0.05 \mathrm{M}$ and $0.5 \mathrm{M}$ (Figure 53). In the second, the TTA was kept constant at $0.25 \mathrm{M}$ while the $\mathrm{HCl}$ was varied between $0.05 \mathrm{M}$ and $0.5 \mathrm{M}$ (Figure 54). The gamma activity in each phase was assayed and the distribution coefficient was calculated.

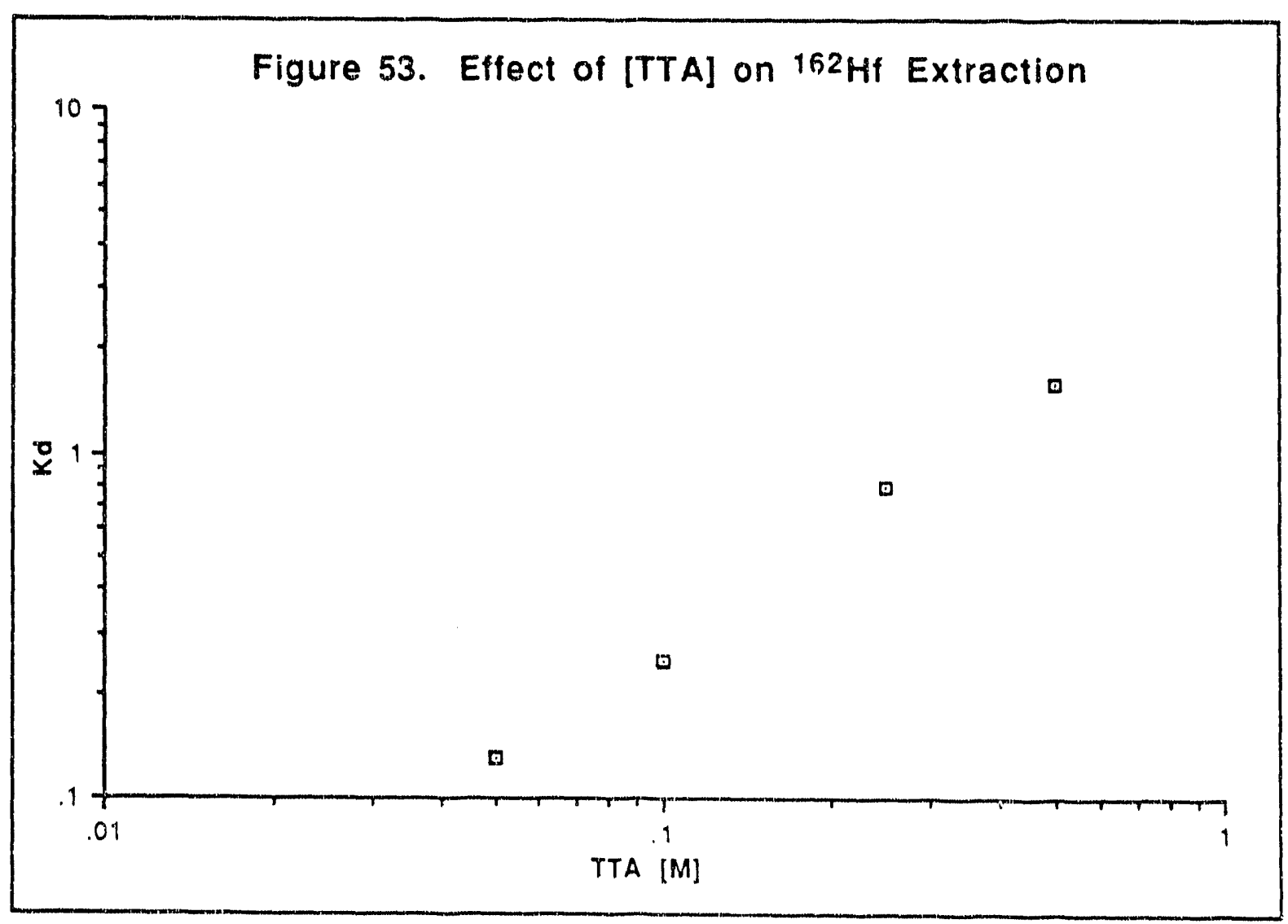




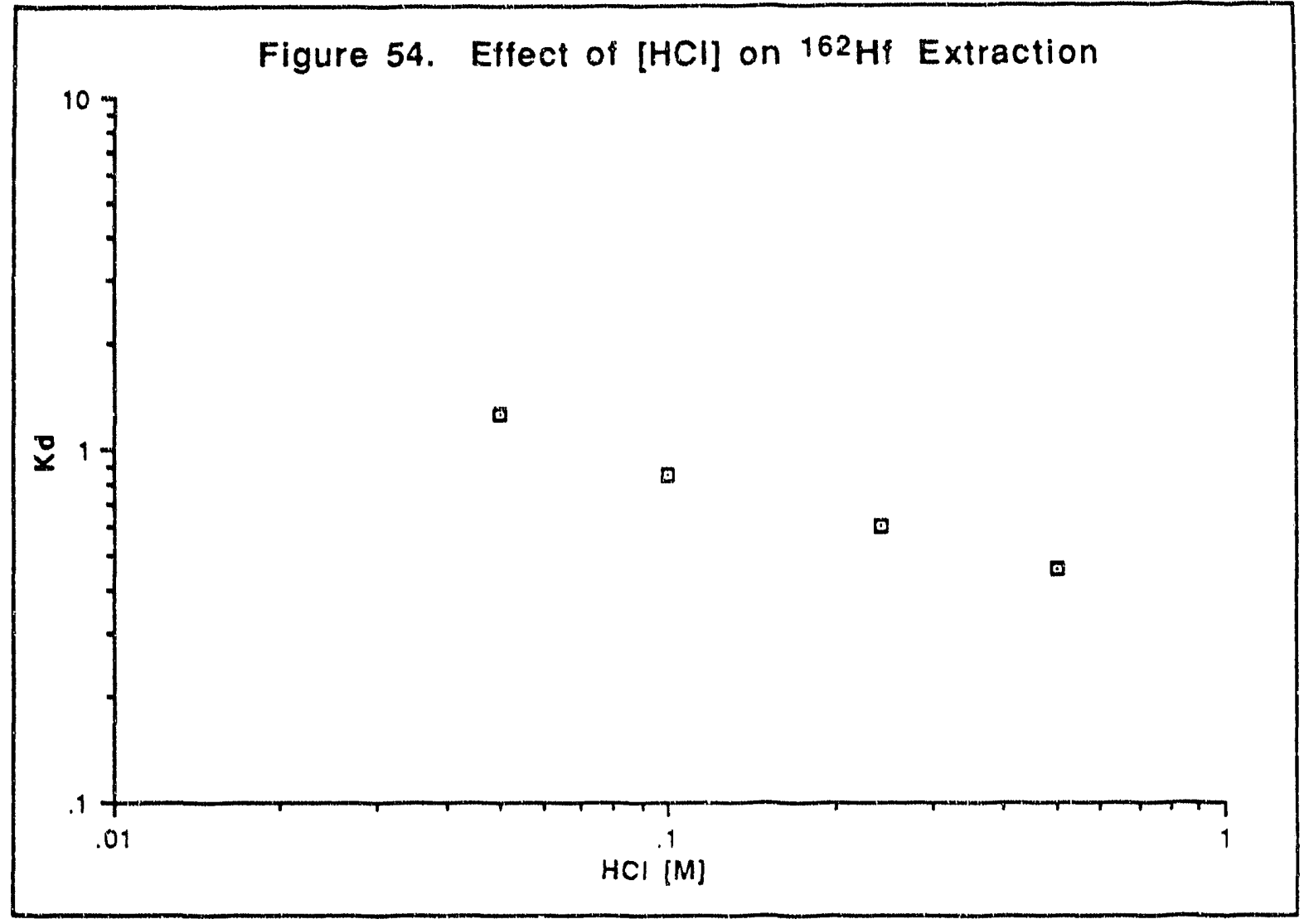

\section{3. $95 \mathrm{Zr}$ and ${ }^{162} \mathrm{Hf}$ Extraction Results}

A comparison of the data for zirconium and hafnium shows three things. First, zirconium generally extracts better than hafnium as indicated by the higher $K_{d}$ values. This result is consistent with the data in the literature [POS61], [CON49]. Second, both zirconium and hafnium form similar complexes with TTA. This is shown by the fact that their slopes as a function of TTA concentration are similar (both approximately 1.2), which means they are similarly dependent on the TTA concentration (See Figures 51 and 53). From these figures, it can be seen that a good choice for the range of TTA concentrations for rutherfordium extraction would be between about $0.25 \mathrm{M}$ and $0.5 \mathrm{M}$. In addition, TTA is kinetically slow compared to TIOA and TBP [POS61]. A high TTA concentration helps overcome the slow kinetics. Therefore, $0.5 \mathrm{M}$ TTA in benzene was the concentration chosen for rutherfordium extraction. 
Finally, the need for a low acid concentration for good extraction is shown in Figures 52 and 54 . This is especially true if rutherfordium behaves more like plutonium than the other Group 4 elements. Previous work has shown that the Group 4 elements have higher distribution coefficients than plutonium under the same conditions [POS61]. In addition, the equilibrium constants for TTA complex formation of plutonium are lower than for hafnium or zirconium [STA64]. From these zirconium and hafnium results, $\mathrm{HCl}$ solutions of $0.24,0.10$, and $0.05 \mathrm{M} . \mathrm{HCl}$ solutions were chosen for extraction studies of rutherfordium.

\section{Rutherfordium and Homolog Extraction Results}

Studies of the rutherfordium homologs were carried out using ${ }^{95} \mathrm{Zr},{ }^{172} \mathrm{Hf}$, ${ }^{228} \mathrm{Th}$, and ${ }^{238} \mathrm{Pu}$ tracers. All solutions were pre-equilibrated prior to the experiments. The homolog experiments were conducted with $500 \mu \mathrm{L}$ each of the aqueous and organic phases. The $\mathrm{HCl}$ concentration was varied from 0.05 $\mathrm{M}$ to $0.24 \mathrm{M}$ and contained the radiotracer. The organic phase was $0.5 \mathrm{M}$ TTA in benzene.

The aqueous and organic phases were vigorously mixed for 2 minutes, and centrifuged for 30 seconds to separate the phases. The phases were extracted and assayed for activity as described in Section 2.C and the percent extraction or distribution coefficients were calculated.

The rutherfordium production and chemistry was done at the 88 -Inch Cyclotron (see Section 2.A.4.a). At the collection site, the ${ }^{261} \mathrm{Rf}$ with the $\mathrm{KCl}$ aerosol was collected on a Teflon disk for 90 seconds. The $261 \mathrm{Rf}$ was removed from the Teflon disk using $10 \mu \mathrm{L}$ of aqueous phase and was added to $20 \mu \mathrm{L}$ of organic phase in a $1 \mathrm{~mL}$ centrifuge cone and mixed in a sonicator for 10 seconds. The solution was centrifuged for 10 seconds to separate the phases. The organic phase was removed and placed on a glass cover slip on a hot 
plate to evaporate the organic phase, leaving the 261Rf on the cover slip. The sample was assayed as described in Section 2.C.

The results (Figure 55) show that of the elements studied rutherfordium extracts greater only than thorium in the $\mathrm{HCl}$ range examined. The extraction of zirconium, hafnium, plutonium, and thorium follows the values in the literature [RAM78], [BAG76], [SEK67], [STA64], [POS61]. Zirconium extracted the best. Hafnium and plutonium extracted about the same, with hafnium usually slightly greater. Rutherfordium extracted at a percentage between plutonium and thorium. Thorium extraction was the lowest.

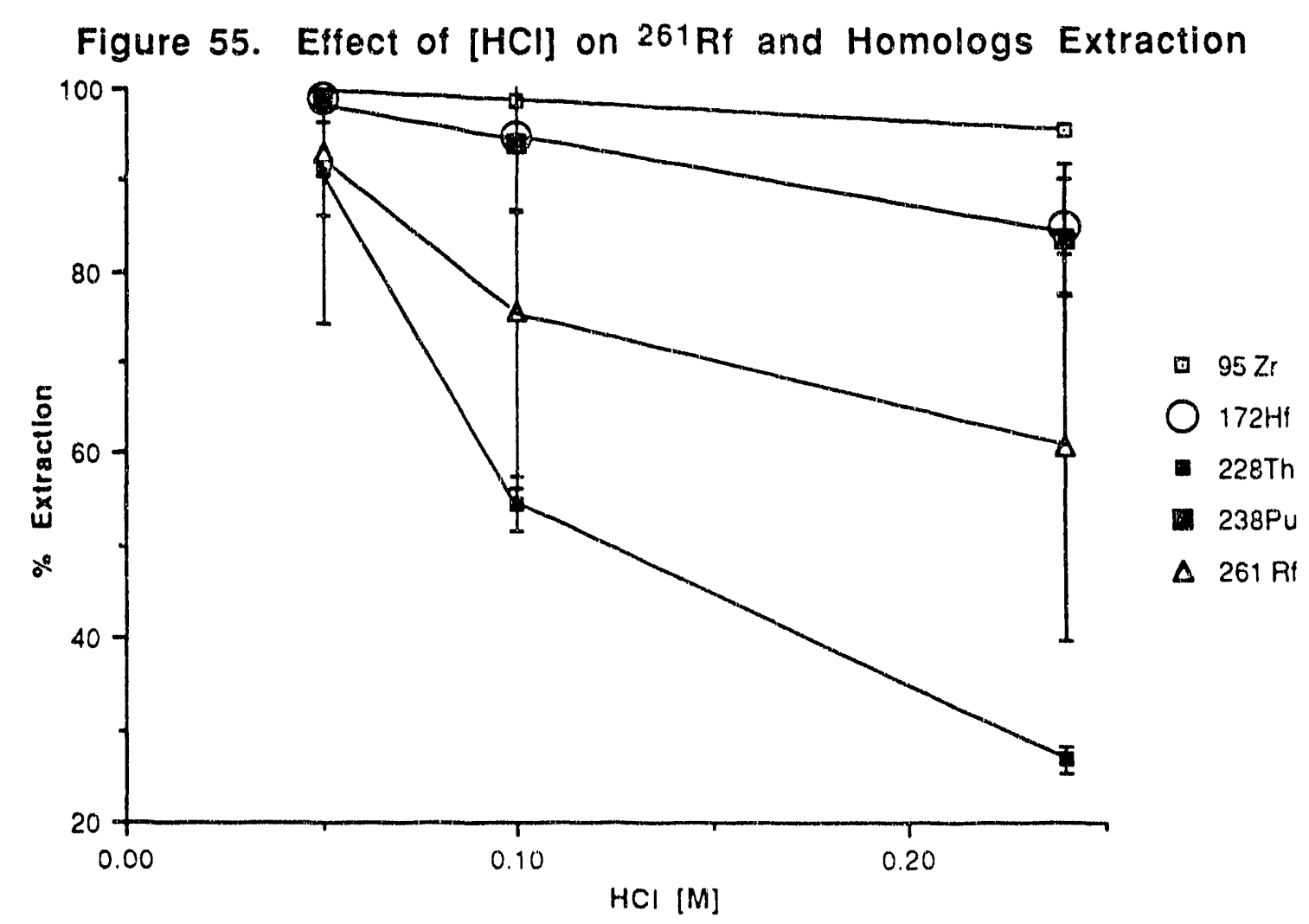

Extraction of all the tetravalent tracers increased as the $\mathrm{HCl}$ concentration decreased. At the lowest $\mathrm{HCl}$ concentration $(0.05 \mathrm{M})$, the extraction for all the tracers was greater than $90 \%$. There was very little trivalent actinide extraction 
under the conditions studied. In fact, under these conditions, TTA gave the better separation from the trivalent actinides compared to TIOA or TBP. TTA begins to extract the trivalent lanthanides and actinides near $\mathrm{pH}=3$ [SEK67] [STA64]. Extractions with an organic phase of 0.5 M TTA in benzene and an aqueous phase of $0.05 \mathrm{M} \mathrm{HCl}$ would be optimal for new rutherfordium isotope searches. These conditions would eliminate contamination from trivalent actinides which could give spurious SF or alpha events.

\section{Calculations}

Equation 7 shows the interrelationship between $K_{d}$ and $K_{\text {eq }}$ for a metal extraction. However, this relationship is true only if the form of the metal complex is the same. In the $\mathrm{HCl}$ range examined, it is assumed that the extracted complex for plutonium, thorium, hafnium and zirconium is the same [RAM78], [BAG76]. However, the literature also contains instances where zirconium has different forms (see Section 3.E.1). For these calculations, it is assumed that the TTA-Rf complex is the same as the other tetravalent metal species.

The calculations will be used to examine the distribution coefficient of the metals at $0.24 \mathrm{M}$ and $0.10 \mathrm{M} \mathrm{HCl}$. The rutherfordium extraction at $0.05 \mathrm{M} \mathrm{HCl}$ was too high to calculate a meaningful distribution coefficient. Plots of $K_{d}$ versus $K_{\text {eq }}$ for the tracers are used to determine the equilibrium constant for the TTA-Rf complex. Table 19 shows the distribution coefficients calculated for the metals at $0.1 \mathrm{M}$ and $0.24 \mathrm{M} \mathrm{HCl}$.

The equilibrium constant for the tracers with TTA are presented in Table 20 The equilibrium constants for TTA reported in the literature vary [STA64], [MAR69C], [KAT86], [PAT80] The values for zirconium, hafnium, and plutonium varied by about a factor of 10 . The equilibrium constant for thorium varied by more than a factor of 100 . The values used for the calculations utilized TTA 
equilibrium constants derived in weak acid solutions [PAT80]. The graph of log $K_{d}$ versus log $K_{e q}$ is shown in Figure 56.

\begin{tabular}{|lll|}
\hline \multicolumn{2}{|c|}{ Table 19. Distribution Coefficients for 261 Rf and Homologs } \\
Isotope & $\mathrm{K}_{\mathrm{d}}(0.24 \mathrm{M} \mathrm{HCl})$ & $\mathrm{K}_{\mathrm{d}}(0.10 \mathrm{M} \mathrm{HCl})$ \\
$95 \mathrm{Zr}$ & $21.0 \pm 1.4$ & $70.2 \pm 4.7$ \\
$172 \mathrm{Hf}$ & $5.60 \pm 0.37$ & $17.4 \pm 1.5$ \\
$228 \mathrm{Th}$ & $0.367 \pm 0.020$ & $1.20 \pm 0.07$ \\
$238 \mathrm{Pu}$ & $5.10 \pm 0.29$ & $15.8 \pm 1.2$ \\
$261 \mathrm{Rf}$ & $0.78 \pm 0.16$ & $1.6 \pm 0.3$ \\
\hline
\end{tabular}

Table 20. TTA Equilibrium Constants

Element

$\mathrm{Zr}$

$\mathrm{Hf}$

Th

$\mathrm{Pu}$

References [PAT80], [STA64], [KAT86]

From Figure 56, the equilibrium constant for rutherfordium with TTA can be calculated and is presented in Table 21. The results show that the equilibrium constant for rutherfordium is between that of plutonium and thorium. $\log \mathrm{K}_{\mathrm{eq}}$

9.30

7.20

2.25

6.85

\begin{tabular}{lc|} 
& Table 21. Keq for Rutherfordium with TTA \\
Solution & $\log \mathrm{K}_{\text {eq }}$ \\
$0.24 \mathrm{M} \mathrm{HCl}$ & $3.58 \pm 0.76$ \\
$0.10 \mathrm{M} \mathrm{HCl}$ & $2.77 \pm 0.54$ \\
Ave & $3.18 \pm 0.90$ \\
\hline
\end{tabular}




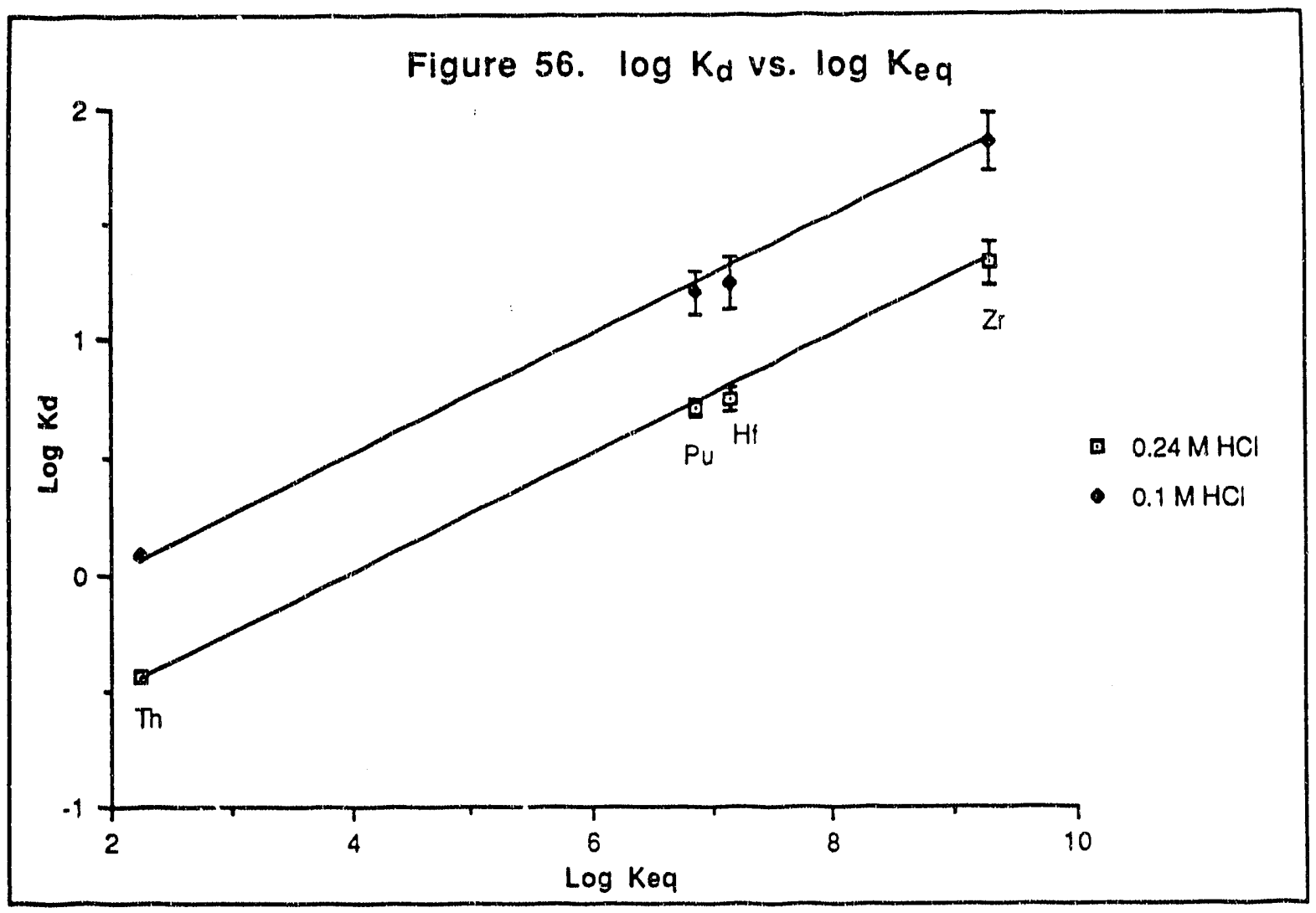

TTA extraction studies have been extensively used to study the hydrolysis constants of metals [BAE76]. This is due to the fact that TTA metal complexation simulates metal hydrolysis, since oxygen is the donor group in TTA. The reaction for hydrolysis of tetravalent metal is:

$$
x M^{4+}+y H_{2} O \leftrightarrow M_{x}\left(O H^{-}\right) y(4 x-y)++y H^{+}
$$

The calculation for rutherfordium hydrolysis will ignore the formation of polymers. Polymer formation is highly unlikely due to the low production rate. Therefore, $x=1$ for Equation 8. From Equation 8, the hydrolysis constants are:

$$
K_{1 y}=\frac{\left[M(O H) y^{(4-y)+}\right]\left[H^{+}\right]^{y}}{\left[M^{d+}\right]}
$$


where $y$ is the number of hydroxide groups on the metal. The hydrolysis constants of the tetravalent tracers are presented in Table 22. The plot of Khyd versus $K_{e q}$ is shown in Figure 57.

\begin{tabular}{|lcccc|}
\hline & Table 22. & \multicolumn{4}{c|}{ Tracer Hydrolysis Constants } \\
Element & $\log K_{11}$ & $\log K_{12}$ & $\log K_{13}$ & $\log K_{14}$ \\
$\mathrm{Zr}$ & 0.3 & -1.7 & -5.1 & -9.7 \\
$\mathrm{H} f$ & -0.25 & -2.4 & -6.0 & -10.7 \\
$\mathrm{Th}$ & -3.20 & -6.93 & -11.7 & -15.9 \\
$\mathrm{Pu}$ & -0.5 & -2.3 & -5.3 & -9.5 \\
\hline
\end{tabular}

Reference [B $\overline{A E 76]}$

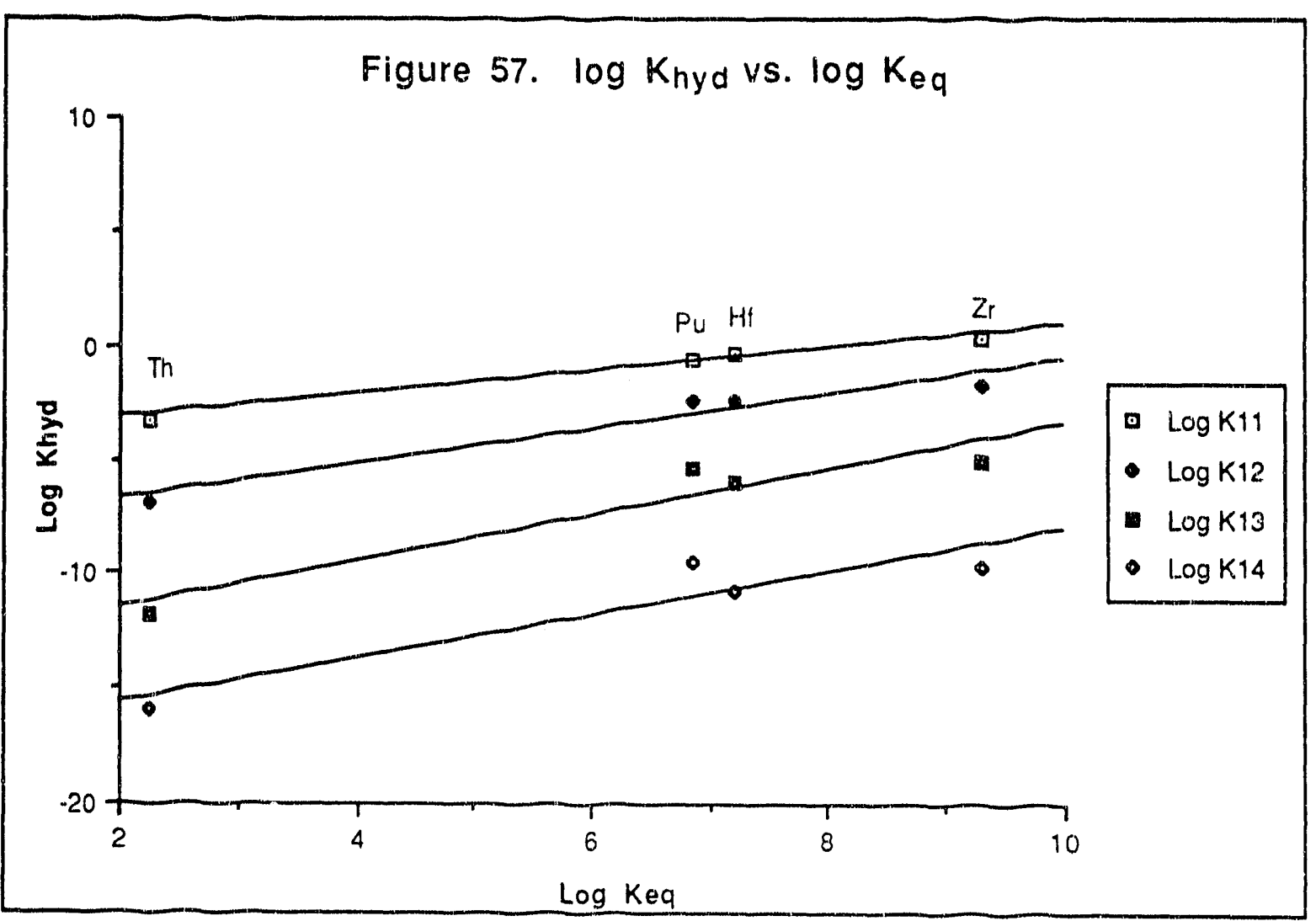

Figure 57 shows the relationship between $K_{\text {hyd }}$ and $K_{\text {eq. }}$. However, the correlation between $\log K_{e q}$ and $\log K_{h y d}$ decreases as the hydrolysis number 
increases. The reason for this is the fast decrease in the hafnium hydrolysis constant. Table 22 shows that $\mathrm{K}_{11}$ for hafnium is greater than that of pluionium. However, $K_{13}$ and $K_{14}$ for hafnium are less than that of plutonium. This decreases the correlation between $K_{h y d}$ and $K_{e q}$. The equations for the four lines in Figure 57 were used to calculate the hydrolysis constants for rutherfordium. These results are presented in Table 23.

Table 23. Rutherfordium Hydrolysis Constants

$X Y$

11

12

13

14 $\log K_{x y}$

$-2.6 \pm 0.7$

$-5.9 \pm 1.7$

$-10.2 \pm 2.9$

$-14.5 \pm 4.1$

These results indicate that rutherfordium will not hydrolyze under conditions when zirconium and hafnium will. Therefore, rutherfordium chemistry can be studied in lower acid concentrations compared to zirconium chemistry. The chemistry of zirconium and hafnium is often limited by hydrolysis.

The hydrolysis constant of rutherfordium is greater than the hydrolysis constant of thorium. Rutherfordium should experience hydrolysis in slightly more acidic solution than thorium. Thorium hydrolysis, in $10^{-5} \mathrm{M}$ concentration of thorium, is not dominant until $\mathrm{pH}=4$. Studies of rutherfordium chemistry up to this $\mathrm{pH}$ range should be possible without interference from hydrolysis. Zirconium and hafnium would only exist as hydrolysis products under these weak acid conditions [BAE76]. This result further helps define an acceptable range of $\mathrm{HCl}$ concentrations for studies of rutherfordium. 
The hydrolysis constants were used to examine the hydrolysis species distribution as a function of pH for zirconium, hafnium, thorium, plutonium, and rutherfordium (Figures 58-62). The species distribution was calculated using the program SPCONC, which is a subroutine of the program BETA [HAR81], [HAR79]. The species were calculated for a $1 \mathrm{nM}$ metal solution. The results show that zirconium and hafnium will exist as hydrolysis products in conditions where rutherfordium will not.

Finally, a calculation of the ionic radius can be made utilizing the equilibrium constant derived for rutherfordium with TTA. Born [BOR20] proposed that if ions are assumed to be spherical, the energy of solution should be expressed by the equation:

$$
\Delta E=\frac{e^{2}}{2 r}\left(1-\frac{1}{D}\right)
$$

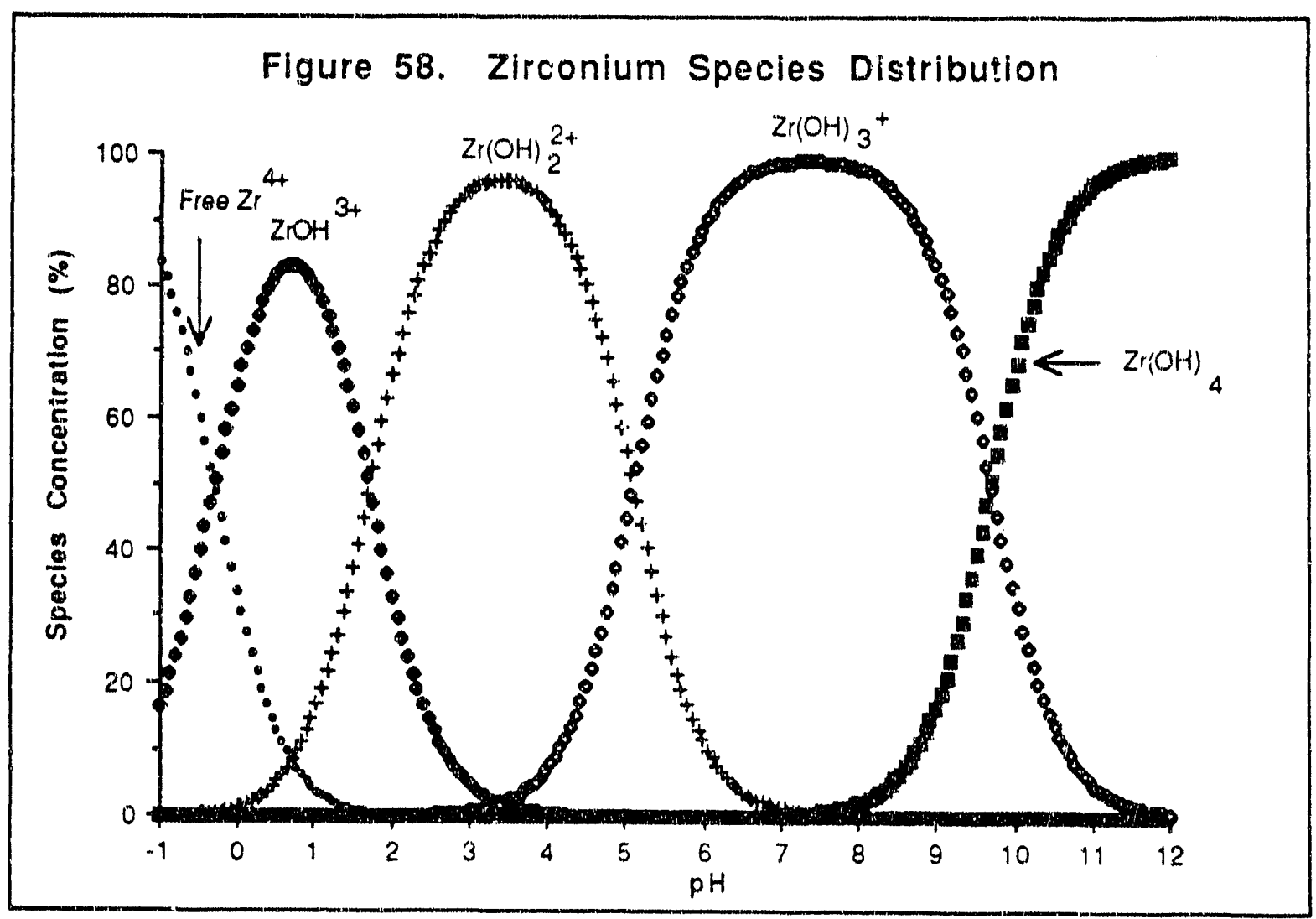




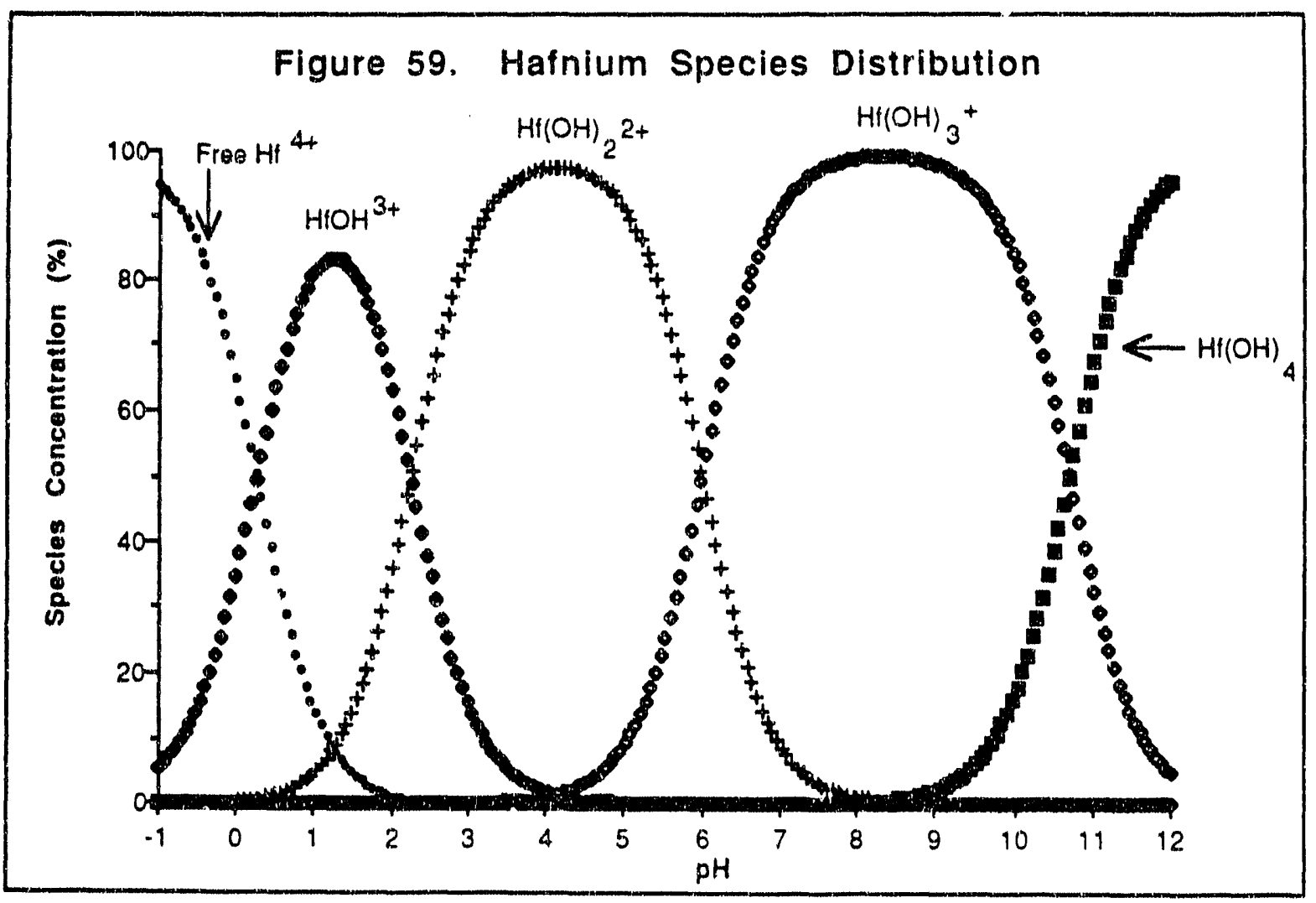

Figure 60. Thorium Species Distribution

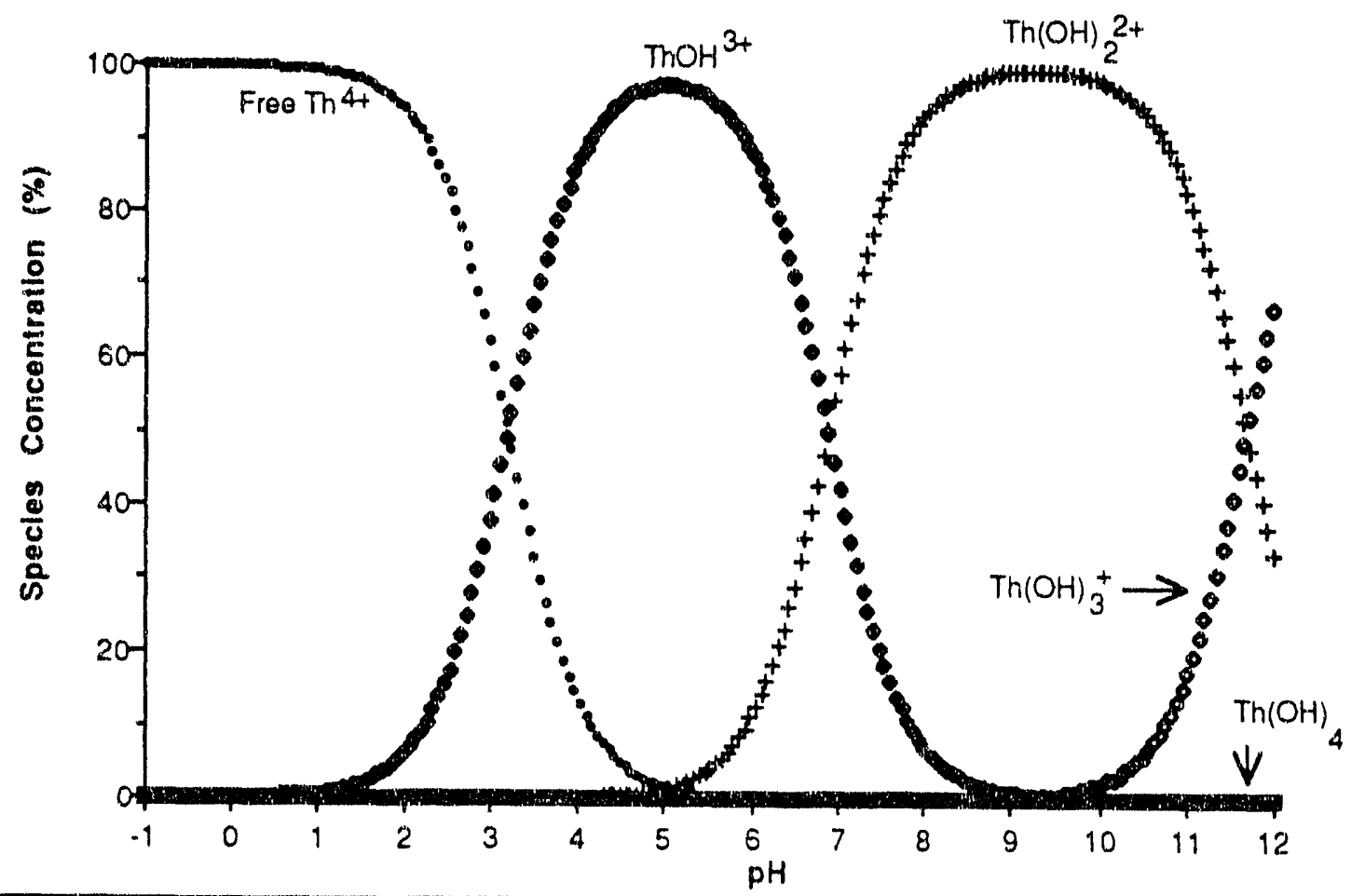



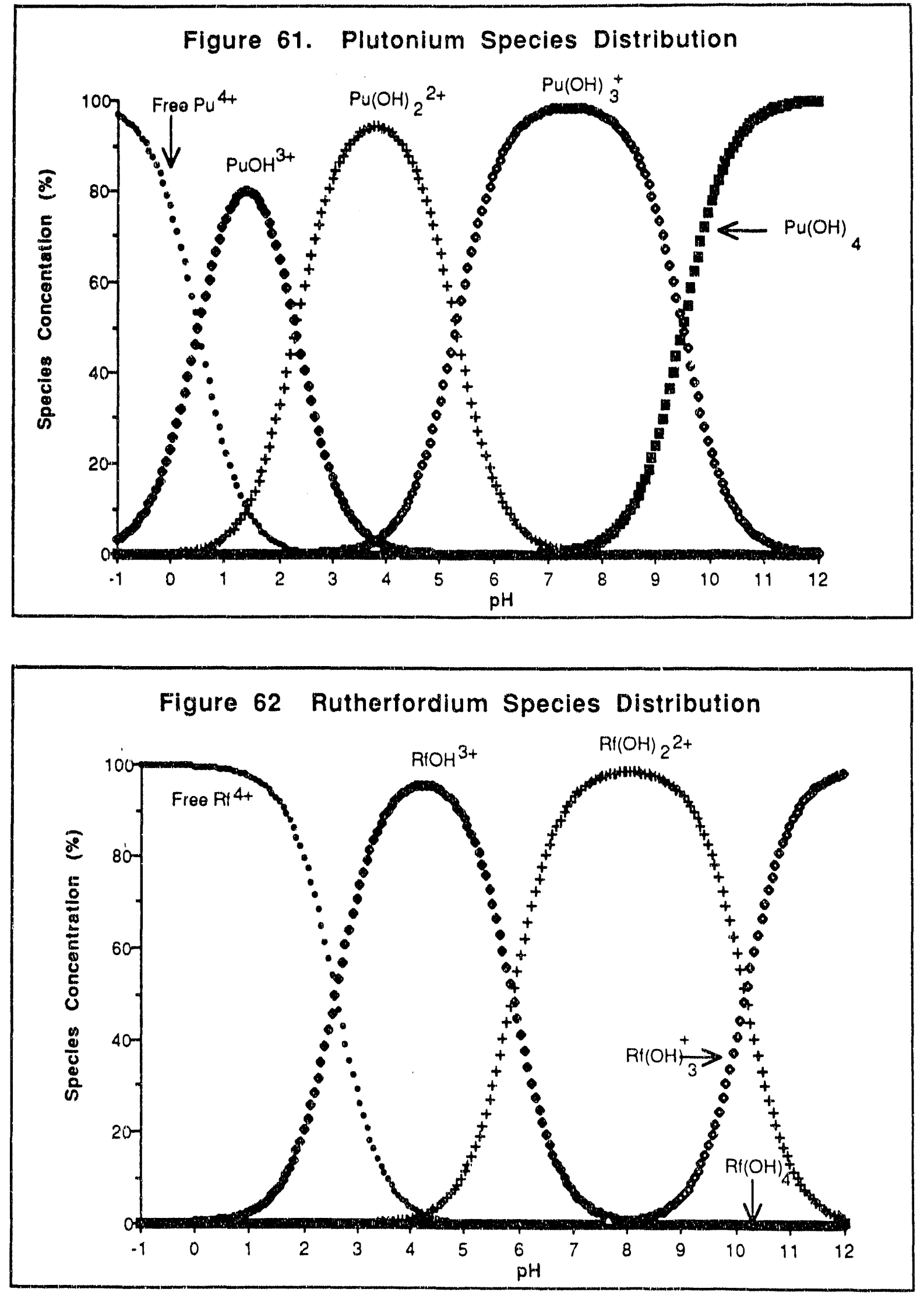
where $D=$ dielectric constant of the solvent, $r=$ radius of the ion, $e=$ charge on the ion, and $\Delta E=$ energy change. Since $\log K_{e q}$ is directly related to energy [MAR52], Equation 10 can be rearranged to give the proportionality:

$$
\log K_{e q} \alpha \frac{e^{2}}{r}
$$

In general, this equation implies an increase in the equilibrium constant with an increase in $\mathrm{e}^{2 / \mathrm{r}}$. If Equation 11 is applied to the tetravalent metals used in the extraction experiments, $e^{2}$ can be neglected and the proportionality simply becomes:

$$
\log K_{e q} \alpha \frac{1}{r}
$$

Therefore, plotting $1 / r$ versus log $K_{\text {eq }}$ should show the relationship between ionic radius and $\log \mathrm{K}_{\text {eq. }}$. This plot is shown in Figure 63; the ionic radius used for the tracers is given in Table 8.

Although Figure 63 is not a linear relationship, it is strikingly consistent with the graphs presented in the literature [MAR52]. The ionic radii for both the 6 and 8 coordinate complexes are included on the figure. The ionic radii of rutherfordium was estimated from the figure using the calculated equilibrium constant of rutherfordium and is presented in Table 24. The error in the estimate results from the error in the $\log \mathrm{Keq}_{\mathrm{eq}}$ for rutherfordium.

The ionic radius estimates can be compared to values in the literature. $A$ previous estimate for the ionic radius of 6 coordinate rutherfordium from experimental data was $89 \mathrm{pm}$ [GLE89]. An estimates of $80 \mathrm{pm}$ was obtained for 6-coordinate rutherfordium using MCDF calculations [JOH90]. The estimate of 
$91 \pm 4 \mathrm{pm}$ obtained in these experiments is in good agreement with the previous experimental value. No previous ionic radius estimate of 8-coordinate rutherfordium has been made, but the value of $102 \pm 4 \mathrm{pm}$ appears reasonable.

The $12 \%$ increase in the ionic radius of rutherfordium from 6 -coordinate to 8 coordinate is within an acceptable increase for the tetravalent elements examined (Table 25).

Table 24. Ionic Radius for Tetravalent Rutherfordium

Coordination Number Ionic Radius (pm)

6

$91 \pm 4$

8 $102 \pm 4$

Figure 63. $1 / r$ vs. $\log K_{e q}$

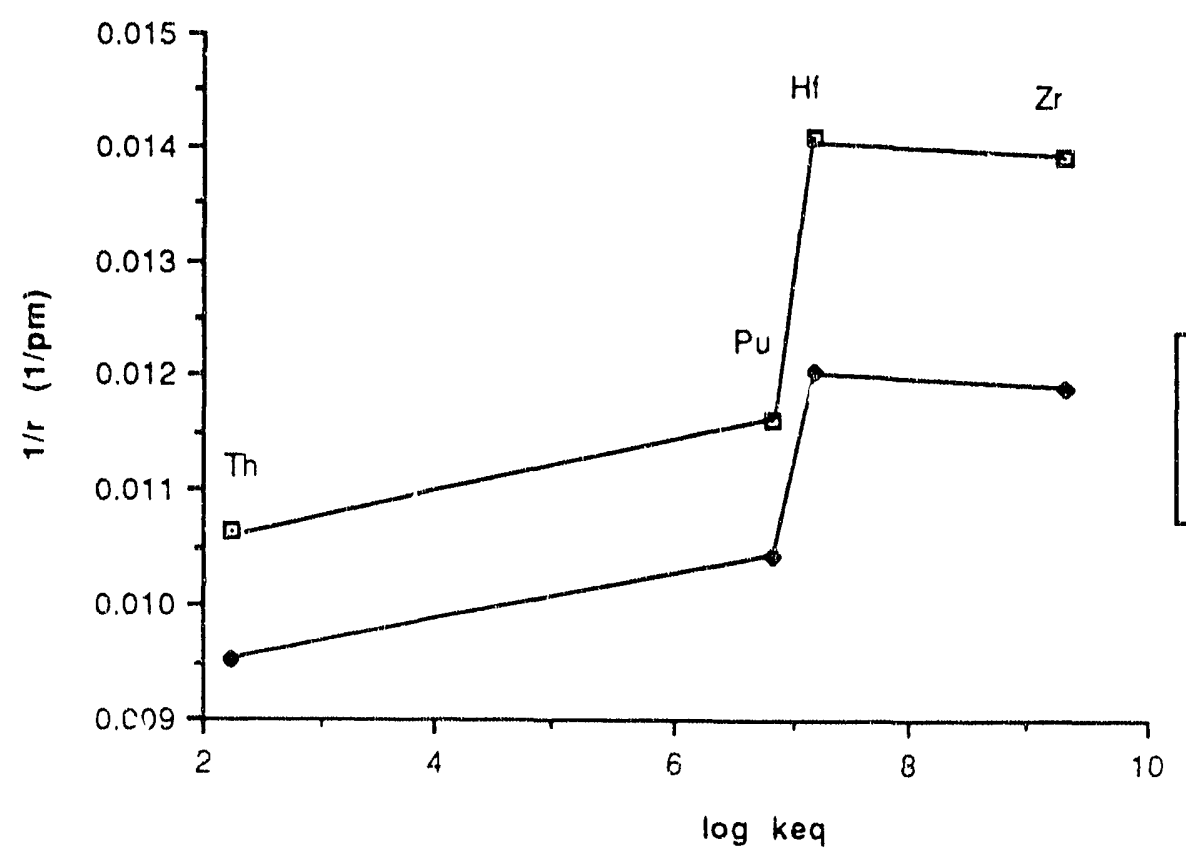




\begin{tabular}{|lc|}
\hline \multicolumn{2}{|c|}{ Table 25. Increase in Ionic Radius from 6-Coordinate to 8 - } \\
Coordinate \\
Element \\
Zr & Ionic Radius Increase (\%) \\
$\mathrm{Hf}$ & 16.7 \\
$\mathrm{Th}$ & 16.9 \\
$\mathrm{Pu}$ & 11.7 \\
$\mathrm{Rf}$ & 11.6 \\
\hline
\end{tabular}

Reference[SHA76] 


\section{Chapter 4. Search for $\underline{263} \underline{\text { Rf }}$}

\section{A. Introdustion}

The decay properties of the heavy element isotopes with neutron number larger than 157 are of great interest. These heavy, neuiron rich isotopes will have lower $Q$ values for alpha and EC decay compared to the lighter isotopes. The $Q$ value is the energy of the reaction. The lower $Q$ values will result in an increase in half-life. Recent discoveries of $260 \mathrm{Md}$ and $261 \mathrm{~L} . \mathrm{r}$ indicate that the odd neutron hindrance against SF is great enough that the overall half-lives of these neutron rich heavy element isotopes are on the order of minutes or longer [LOU87], [LOU86]. Due to calculated smaller $Q_{\alpha}$ and $Q_{E C},{ }^{263} \mathrm{Rf}$ is predicted to have a longer half-life than ${ }^{261} \mathrm{Rf}$ for these decay modes. If it can be produced and identified, the longer lived ${ }^{263} \mathrm{Rf}$ could be used for more complex chemical studies. In addition, measurement of the decay properties of $263 \mathrm{Rf}$ will help determine the importance of the deformed nuclear shell predicted near $Z=110$ and $N=162$ [MÖL88].

Past searches for ${ }^{263 R f}$ yielded no events, with a calculated upper limit of 20 minutes for the half-life [BRU89]. These experiments, however, were hindered by a long chemical separation time (up to 20 minutes). Liquid-liquid extractions can be employed to reduce the chemistry time to about 1 minute.

From the experiments conducted with $261 \mathrm{Rf}$, the TTA extraction system gives a high rutherfordium extraction with little trivalent actinide contamination (see Section 3.E.4). The organic phase for the ${ }^{263} \mathrm{Rf}$ studies was $0.5 \mathrm{M}$ TTA in benzene, and the aqueous phase was $0.05 \mathrm{M} \mathrm{HCl}$. The volumes used were 20 $\mu \mathrm{L}$ of organic and $10 \mu \mathrm{L}$ of aqueous. The irradiation and collection time was 3 minutes. The procedure is similar to the TTA extraction studies with $261 \mathrm{Rf}$ (see Section 3.E.4.). 


\section{B. Calculations}

\section{Cross Section}

In previous attempts to produce $263 \mathrm{Rf}$, the reaction ${ }^{248} \mathrm{Cm}(22 \mathrm{Ne}, \alpha 3 n)$ was used [BRU89]. The search for ${ }^{263}$ Rf presented in this section used the ${ }^{248} \mathrm{Cm}\left({ }^{18} \mathrm{O}, 3 n\right)$ production reaction. This reaction was chosen because previous heavy element production work with the $(22 \mathrm{Ne}, \alpha 3 \mathrm{n})$ reaction gave no results [KRA92], [BRU89].

The cross section for the ${ }^{248} \mathrm{Cm},\left({ }^{18} \mathrm{O}, 3 \mathrm{n}\right)$ reaction was calculated using a modified version of the SPIT code [ALO74]. There are literature reports that the cross section for an alpha $3 n$ reaction can be approximated by a $4 n$ reaction [KRA92]. For this instance, the $248 \mathrm{Cm}(190,4 \mathrm{n})$ reaction was calculated with SPIT to approximate the ${ }^{248} \mathrm{Cm}$ (22Ne, a3n) reaction. Figure 64 shows the SPIT calculations for the ${ }^{248} \mathrm{Cm}(22 \mathrm{Ne}, \alpha 3 n){ }^{263} \mathrm{Rf} ;{ }^{248} \mathrm{Cm},(18 \mathrm{O}, 3 n){ }^{263} \mathrm{Rf}$; and ${ }^{248} \mathrm{Cm},(180,5 n)^{261} \mathrm{Rf}$ reactions. The calculations for the $3 \mathrm{n}$ reactions are usually optimistic by an order of magnitude. Therefore, the $3 n$ cross section values were reduced by an order of magnitude. The peak of the $3 n$ excitation function is at $92.5 \mathrm{MeV}$. At this energy, the cross section is estimated to be $300 \mathrm{pb}$ after reduction by 10 . The alpha $3 n$ reaction was estimated to have the highest cross section of $9 \mathrm{nb}$. However, this reaction was not used due to the previous unsuccessful experiments which used this production method.

The $3 \mathrm{n}$ cross section of $300 \mathrm{pb}$ can be used to estimate the ${ }^{263} \mathrm{Rf}$ production rate. With a ${ }^{1805+}$ beam intensity of $0.5 \mathrm{p \mu A}$ and a ${ }^{248} \mathrm{Cm}$ target thickness of $500 \mu \mathrm{g} / \mathrm{cm}^{2}$, the production rate is about 2 atoms per hour. Folding in the gas jet transport yield, chemical yield and detector efficiencies, a detection rate of 1.3 events per hour for SF and 0.7 events per hour for alpha decay is estimated. 


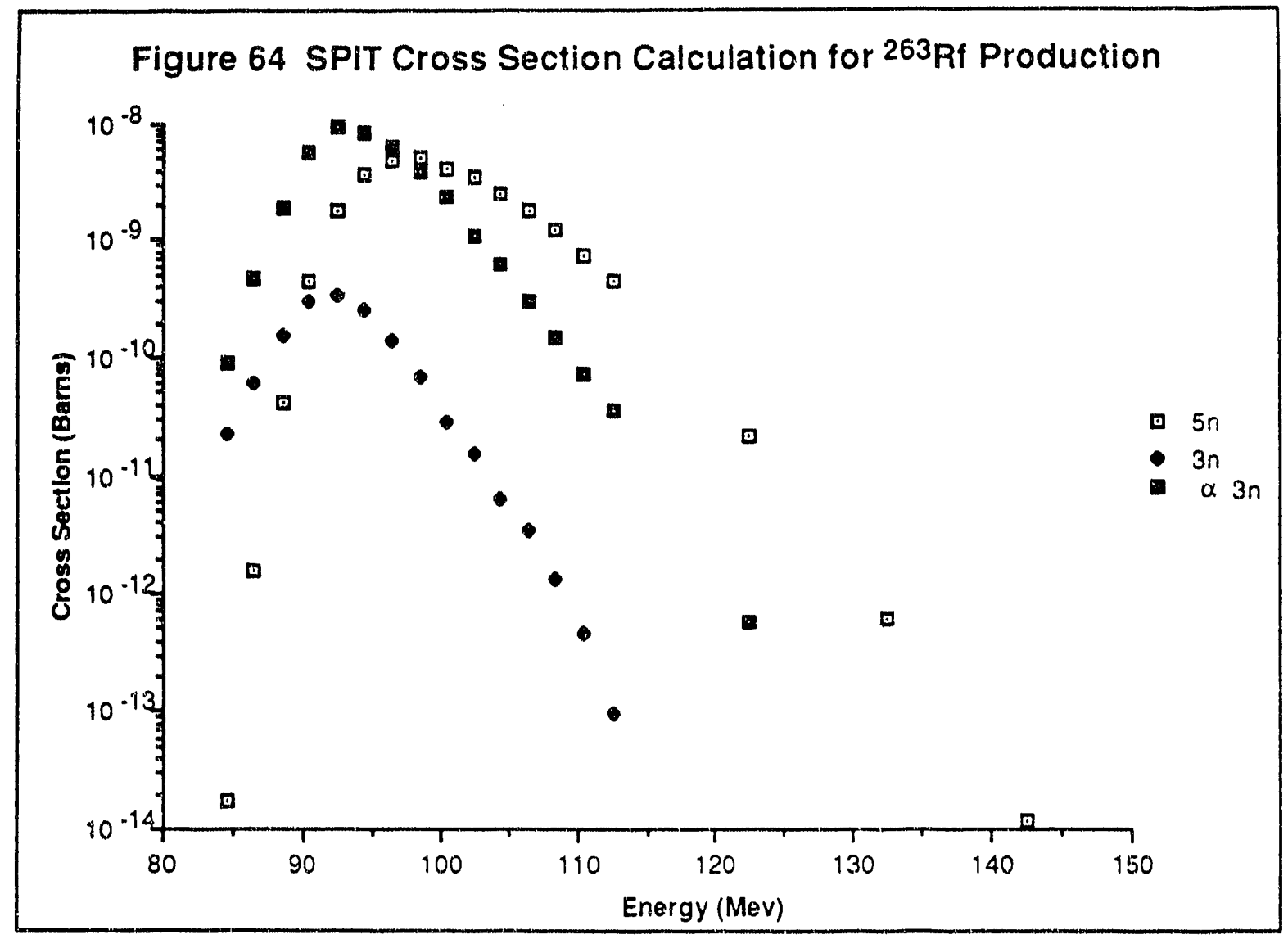

\section{Alpha Decay}

To calculate the alpha decay half-life, the masses of the parent and daughter need to be derived. The masses are used to calculate the energy release $(Q$ value) for a nuclear decay process from the equation:

$$
x P \rightarrow(x-4) D+\alpha+Q
$$

where $P$ is the parent and $D$ is the daughter. Knowing the mass of $P, D$, and $\alpha$; $Q$ can be obtained.

There are a variety of mass estimates for the heavy elernents [HAU88]. To determine which masses are most suitable for ${ }^{263} \mathrm{Rf}$, it is useful to compare the estimates with the experimental values for ${ }^{261} \mathrm{Rf}$. The experimental $Q$ value for 
the alpha decay of ${ }^{261} \mathrm{Rf}$ is $8.60 \mathrm{MeV}$ [LED78]. The masses which give the best estimate of the $Q$ value of ${ }^{261} R f$ should also give an accurate estimate for ${ }^{263} R f$. The mass estimates are given in mass excess and are measured in units of $\mathrm{MeV}$. The equation for mass excess is:

$$
\Delta=M-A
$$

where $\Delta$ is the mass excess, $M$ is the total atomic mass, and $A$ is the mass number. The mass excess for an alpha particle is $2.425 \mathrm{MeV}$. Once the $Q$ value is calculated, the alpha decay energy can be obtained.

Most of the kinetic energy of alpha decay is carried by the alpha particle. A ratio of the parent and daughter masses determines the amount of energy available to the alpha particle. The equation relating $Q$ value to alpha decay energy $\left(E_{\alpha}\right)$ is:

$$
E_{\alpha}=\frac{A_{D}}{A_{p}}(Q)
$$

where $A_{P}$ is the mass number of the parent, $A_{D}$ is the mass number of the daughter, and $Q$ is the $Q$ value for the alpha decay. Substituting $A_{\alpha}$ for $A_{D}$ in Equation 15 will give the recoil energy of the daughter nucleus. The $Q$ values and alpha decay energies calculated from the various masses are presented in Table 26. From this table, the mass predictions of Satpathy [SAT88] and Möller and Nix [MÖL_88A] give the closest agreement to the experimental $Q$ value for 261 Rf.

The model used by Satpathy [SAT88] calculated 3481 nuclei in the range of $18 \leq A \leq 267$ using the infinite nuclear matter model based on the HugenholtzVan Hove theorem. The infinite nuclear matter approximation is used because 
this model assumes that Coulombic repulsion and surface attractive forces are equal. This postulate states that an isolated sphere such as a nucleus will have the same density as infinite nuclear matter.

\begin{tabular}{|c|c|c|c|c|c|c|c|c|c|}
\hline \multirow[b]{3}{*}{$261 \mathrm{Rf}$} & \multicolumn{9}{|c|}{ Table 26. Q value Calculations } \\
\hline & Groote & Liran & Meyer & Spanier & Satpathy & $\begin{array}{l}\text { Möller } \\
\& \text { Nix }\end{array}$ & Möller & Seeger & Tachibana \\
\hline & 101.88 & 101.34 & 102.77 & 101.41 & 101.21 & 101.83 & 100.34 & 101.5 & 101.19 \\
\hline $263 \mathrm{Rt}$ & 105.32 & 104.92 & 106.07 & 104.99 & 104.61 & 104.64 & 103.29 & 104.4 & 104.41 \\
\hline 257 No & 90.62 & 90.09 & 91.54 & 90.25 & 90.17 & 90.84 & 89.69 & 90.9 & 90.62 \\
\hline${ }^{259} \mathrm{No}$ & 94.27 & 93.92 & 95.04 & 94.11 & 93.92 & 94.36 & 94.34 & 94.2 & 93.62 \\
\hline$Q^{261} \mathrm{Ri}$ & 8.835 & 8.825 & 8.805 & 8.735 & 8.615 & 8.565 & 8.225 & 8.175 & 8.145 \\
\hline$E_{\alpha}{ }^{261} R f$ & 8.700 & 8.590 & 8.670 & 8.601 & 8.483 & 8.434 & 8.099 & 8.050 & 8.020 \\
\hline $\mathrm{Q}^{263} \mathrm{R}^{\prime}$ & 8.625 & 8.575 & 8.605 & 8.455 & 8.265 & 7.855 & 7.525 & 7.775 & 8.365 \\
\hline$E_{\alpha}{ }^{263} R f$ & 8.494 & 8.445 & 8.474 & 8.326 & 8.139 & 7.736 & 7.411 & 7.657 & 8.238 \\
\hline
\end{tabular}

References [GRO76], [LIR76], [MYE76], [SPA88], [SAT88], [MOL88A], [MOL88], [SEE76], [TAC88], [HAU88]

Like many mass equations, this one examines the nucleus as a sum of global and local parameters. The initial equation for the mass calculation is:

$$
E^{F}(A, Z)=E^{S}(A, Z)+h(A, Z)
$$

The $E S(A, Z)$ is the global energy term and $h(A, Z)$ is the local energy term.

The equation derived for the mass calculation from the Satpathy model is:

$$
E^{F}(A, Z)=E(A, Z)+a_{s} A^{2 / 3}+a_{c} \frac{Z^{2}}{A^{1 / 3}}-d(A, Z)+h(A, Z) \text { (Equation 17) }
$$

The terms for Equation 17 are listed below.

$E F(A, Z)$ the ground state energy of a real finite nucleus with mass number $A$ and charge number $Z$.

$E(A, Z)$ is the energy contained in a volume of $A$ and $Z$ 
$a_{s} A^{2 / 3}$ is the surface term

$a_{c} \frac{Z^{2}}{A^{1 / 3}}$ is the Coulombic term

$d(A, Z)$ is a pairing term which varies according to the nucleus.

$$
\begin{aligned}
& d(A, Z)=+\Delta \times A^{-0.5} \text { for even-even nuclei } \\
& d(A, Z)=0 \text { for odd- } A \text { nuclei } \\
& d(A, Z)=-\Delta \times A^{-0.5} \text { for odd-odd nuclei }
\end{aligned}
$$

$h(A, Z)$ is the local residual energy term

The global parameters are $a_{s}, a_{c}$ and $\Delta$. For this equation, they are equal to $25.846,0.841$, and $11.709 \mathrm{MeV}$ respectively. A complete description of this equation can be found in [SAT88]

The model employed by Möller and Nix calculated the ground state masses for 4678 nuclei between 160 to 318122 , and is an improved version of their 1981 model [MÖL81]. As in the Satpathy model, this model uses terms for macroscopic and microscopic parts of the nucleus. The macroscopic term uses a Yukawa-plus-exponential potential. The microscopic term uses a foldedYukawa single-particle potential. Scme of the new features of this model are the incorporation of average pairing strength and the use of experimental uncertainties.

The initial equation for the determination of the total nuclear potential energy is:

$E(Z, N$, shape $)=E_{\text {macr }}(Z, N$, shape $)+E_{\text {micr }}(Z, N$, shape $)$

After introduction of the potentials, this equation becomes: 
$E(Z, N$, shape $)=M_{H} Z+M_{n} N-a_{v}\left(1-\left.k v\right|^{2}\right) A+a_{s}\left(1-\left.k s\right|^{2}\right) B_{1} A^{2 / 3}+c_{0} A^{0}+$ $c_{1} \frac{Z^{2}}{A^{1 / 3}} B_{3}-c_{4} \frac{Z^{4 / 3}}{A^{1 / 3}}+f\left(k_{f} r_{p}\right) \frac{Z^{2}}{A}-c_{a}(N-Z)+E_{w}+E_{p}-a_{e} Z^{2.39}$

The terms for Equation 19 are listed below:

$M_{H} Z+M_{n} N$ is the mass excess of $Z$ hydrogen atoms and $N$ neutrons

$a_{v}\left(1-\left.k v\right|^{2}\right) A$ are the volume and volume-asymmetry energies

$a_{s}\left(1-\left.k s\right|^{2}\right) B_{1} A^{2 / 3}$ are the surface and surface asymmetry energies

$C_{0} A^{\circ}$ is the $A^{\circ}$ energy

$c_{1} \frac{Z^{2}}{A^{1 / 3}} B_{3}$ is the direct Coulomb energy

$c_{4} \frac{Z^{4 / 3}}{A^{1 / 3}}$ is the exchange Coulomb energy

$f\left(k_{i} r_{p}\right) \frac{Z^{2}}{A}$ is the proton form factor correction

$\mathrm{c}_{a}(\mathrm{~N}-\mathrm{Z})$ is the charge asymmetry energy

$E_{W}$ is the Wigner energy and $E_{p}$ is the pairing energy. They are dependent upon the $\mathrm{N}$ and $\mathrm{Z}$ of the nuclei.

$E_{W}$ is:

$W(|1|+1 / A)$ for $Z$ and $N$ odd and equal

$W(|1|+0)$ for all other cases

Ep is:

$+\bar{\Delta}_{p}+\bar{\Delta}_{n}-\delta_{n p}$ for $Z$ and $N$ odd

$+\bar{\Delta}_{p}$ for $Z$ odd and $N$ even

$+\bar{\Delta}_{n}$ for $Z$ even and $N$ odd

+0 for $Z$ and $N$ even

$a_{e} z^{2.3}$ is the energy of the bound electrons

A wide variety of constants are present in this model. A complete description of the model and listing of its constants can be found in [MÖL88A] 
While the two models gave similar predictions for the ${ }^{261} R f Q$ value, the $Q$ values for ${ }^{263} R f$ predicted by these two calculations differ greatly. The difference, $0.41 \mathrm{MeV}$, results in a large difference in the calculated alpha half-life.

The relationship between alpha half-life and decay energy was recognized early in radiation research [GEI11]. This relationship was explained by alpha particio tunneling in the nucleus [CON29], [GAM29]. A large number of formulas have been derived to calculate the alpha half-life from the decay energy [VIO66], [TAA61], [WAP59]. Unfortunately, most formulas are valid only over a limited range of nuclei. For our purpose, the alpha half-life calculation was taken from an empirical alpha half-life equation developed for the heavy elements by Hatsukawa et al. [HAT90], [HAT90A].

This equation is:

$\log T=A(Z) \sqrt{\frac{A_{d}}{A_{p} Q_{\alpha}}}\{\arccos \sqrt{X} \cdot \sqrt{X(1-X)}]-20.446+C(Z, N)$

where:

$$
A(Z)=1.40 Z+\frac{1710}{Z}-47.7
$$

$A_{d}$ is the daughter $A$

$A_{p}$ is the parent $A$

$Q_{\alpha}$ is the $Q$ value for the alpha decay

$$
X=R_{3} / R_{2}=r_{0}\left(A_{d}^{1 / 3}+4 \frac{1 / 3}{2 Z_{d} Q^{2}}\right.
$$

$$
r_{0}=1.2249 \mathrm{fm}
$$

$C(Z, N)$ varies according to the region of the nuclides

$$
\begin{aligned}
& C(Z, N)=[1.94-0.020(82-Z)-0.070(126-N)] \\
& \text { for } 78 \leq Z<82,100 \leq N<126 \\
& C(Z, N)=[1.42 \cdot 0.105(Z-82)-0.067(126-N)
\end{aligned}
$$




$$
\begin{aligned}
& \text { for } 82<Z \leq 90,110 \leq N \leq 126 \\
& C(Z, N)=0 \text { for other regions }
\end{aligned}
$$

Initial values calculated from Equation 20 are valid for ground state to ground state transitions only. For even-even nuclei, the calculations are acceptable. For other cases, such as ${ }^{263} \mathrm{Rf}$, decay hindrance must be considered. A hindrance factor of 2 gives good agreement with the experimental odd $A$ alpha half-life values. The calculated alpha half-lives for ${ }^{261} \mathrm{Rf}$ and ${ }^{263} \mathrm{Rf}$ are presented in Figure 65. The ${ }^{261} \mathrm{Rf}$ calculated hindered half-life at $8.3 \mathrm{MeV}$ is 59 seconds. This is in excellent agreement with the experimental half-life of ${ }^{261} \mathrm{Rf}$ of 65 seconds.

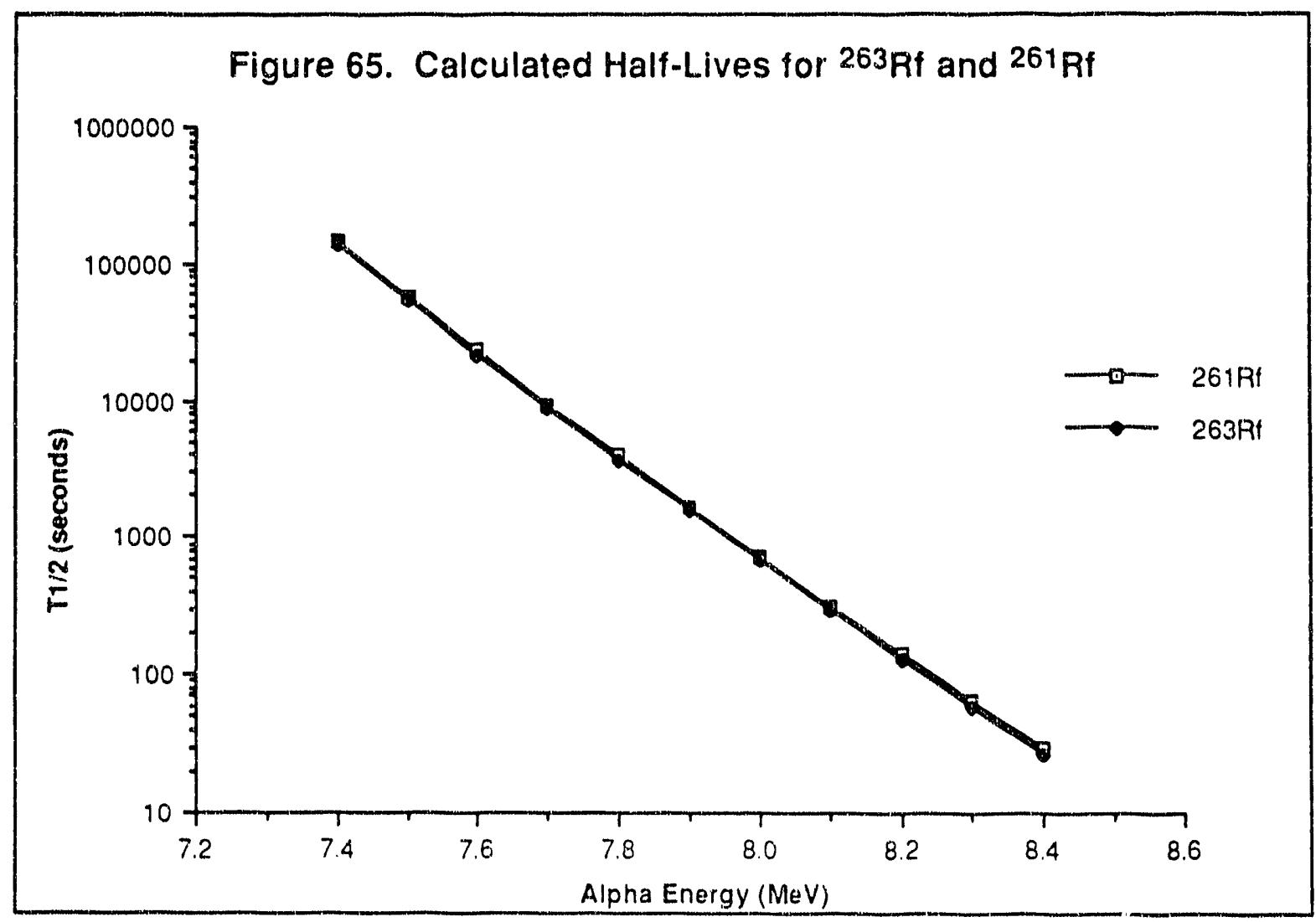

Figure 65 is used to estimate the alpha half-life of 263Rf. As shown in Table 27, half-lives of 222 and 6920 seconds, respectively, are obtained based on 
estimates using the Satpathy and Möller and Nix masses. The $E_{\alpha}$ assumes decay to the ground state. Alpha decay to an excited state will decrease the alpha decay energy and correspondingly increase the half-life.

\section{Table 27. ${ }^{263}$ Rf Alpha Decay Half-Life Estimate}

\begin{tabular}{lll} 
Mass Model & $\mathrm{E}_{\alpha}(\mathrm{MeV})$ & $\mathrm{t}_{1 / 2}$ (sec.) \\
Satpathy & 8.139 & 222 \\
Möller and Nix & 7.736 & 6920 \\
\hline
\end{tabular}

The best estimate is that the alpha decay half-life will be between 100 and 7000 seconds. It should be noted that the Möller and Nix masses are frequently used for heavy element mass calculations. If the Möller and Nix values are better, then the alpha decay half-ife should be at the longer end of the range, or on the order of 2 hours.

\section{Electron Capture}

The electron capture half-life was estimated using the method given in Appendix V of the Table of the Isotopes [LED78]. For this calculation, the QEC and the log $f t$ values for the decay involved need to be estimated. The $Q_{E C}$ from the decay is calculated from the equation:

$$
Q_{E C}=\left(\Delta p \cdot \Delta_{D}\right)
$$

where $\Delta p$ and $\Delta D$ are the mass excess of the parent and daughter. The mass excesses for ${ }^{263} R f$ and $263 \mathrm{Lr}$ from the Satpathy and Möller and Nix masses are presented in Table 28.

The $\log \mathrm{ft}$ is the sum of the logs of the Fermi function and $\log$ of the half-life. For the heavy elements, the $\log \mathrm{ft}$ is approximately 6 [LED78]. Using this value, 
and the QEC values calculated from the masses, the EC half-life was calculated to be about 4000 seconds. The results are presented in Table 28.

\begin{tabular}{|lll|}
\hline \multicolumn{3}{|c|}{ Table 28. Mass Excesses (MeV) of ${ }^{263} \mathrm{Rf}$ and ${ }^{263} \mathrm{Lr}$ and EC Half-lives } \\
& Satpathy & Möller and Nix \\
$263 \mathrm{Rf}$ & 104.61 & 104.64 \\
$263 \mathrm{Lr}$ & 103.31 & 103.01 \\
QEC & 1.30 & 1.63 \\
EC Half-life (seconds) & 4000 & 3600 \\
\hline
\end{tabular}

\section{Spontaneous Fission}

Spontaneous fission (SF) is the predominant decay mode for even-even rutherfordium isotopes. For the odd A rutherfordium isotopes, SF is prominent in the lighter isotopes, 253 and 255. For the heavier odd A isotopes, $257-261$. alpha decay is the dominant decay mode. However, as A increases, the alpha decay energy decreases and hence the half-life increases. At the isotope ${ }^{263} \mathrm{Rf}$, SF decay may compete with alpha decay. Therefore, SF must be considered for a complete estimate of decay modes. The estimate of SF half-life was done using two methods. One method involved the use of a semi-empirical formula for SF half-life calculation by Lojewski and Baran [LOJ88]. The second method utilizes experimental systematics to determine the half-life developed by Hoffman [HOF89].

The following equations are from Lojewski and Baran [LOJ88]. The calculation for the SF half-life uses a single-particle Woods-Saxon potential (Figure 66). In calculating the SF half-life, a semi-empirical mass parameter was used. The mass parameter, $B_{r}$, was calculated from: 


$$
B_{r}=\mu\left[1+\frac{17}{15} k \exp \left[-\left(r-0.75 R_{0} / d\right)\right]\right.
$$

where $\mu$ is the reduced mass of the fission fragments, $R_{0}=r_{0} A^{1 / 3}, ; d=R_{0} / 2.452$, and $k$ is an adjustable parameter set to 11.5 for these calculations. The SF halflife was calculated according to the WKB approximation.

$$
T_{S F}=\frac{\ln 2}{n} \frac{1}{P}
$$

In Equation 25, $n$ is the number of assaults on the fission barrier per unit time and $P$ is the penetrabiliiy through the potential barrier. Assuming $E_{0}=0.5 \mathrm{MeV}$ for the zero point energy, $n=10^{20.54} \mathrm{~s}$. The barrier penetrability $P$ is:

$$
P=\left(1+\exp \left(2 \int_{r_{1}}^{r_{2}}\left[2 B_{r} / \hbar^{2}\left(V-E_{0}\right)\right]^{1 / 2} d r\right)\right)
$$

The terms for Equation 26 are shown in Figure 66.

The result of these calculations for the SF half-life of $263 \mathrm{Rf}$ is 10,000 seconds. The SF half-lives calculated with this method are generally 3-5 orders of magnitude higher than from other methods [LOJ88]. In addition, the calculated results for known odd $A$ rutherfordium are too high by 1.2 orders of magnitude. This fact requires another estimate for the SF half-life of ${ }^{263} \mathrm{Rt}$.

The experimental systematic method relies upon hindrance factor calculations for the odd neutron and is taken from Hoffman [HOF89]. In the case of $263 \mathrm{Rf}$, the odd neutron is the 159th neutron. The only other known even $Z$ element with 159 neutrons is $259 \mathrm{Fm}$. To calculate the spontaneous fission hindrance (HFSF) for an odd $A$ even $Z$ nuclide, the equation is: 


$$
H F S F=\frac{T_{1 / 2}\left({ }^{A} Z\right)}{\left[T_{1 / 2}\left({ }^{A-1} Z\right) \times T_{1 / 2}\left({ }^{A+1} Z\right)\right]^{1 / 2}}
$$

\section{Figure 66. Woods-Saxon Potential Diagram For Protons}

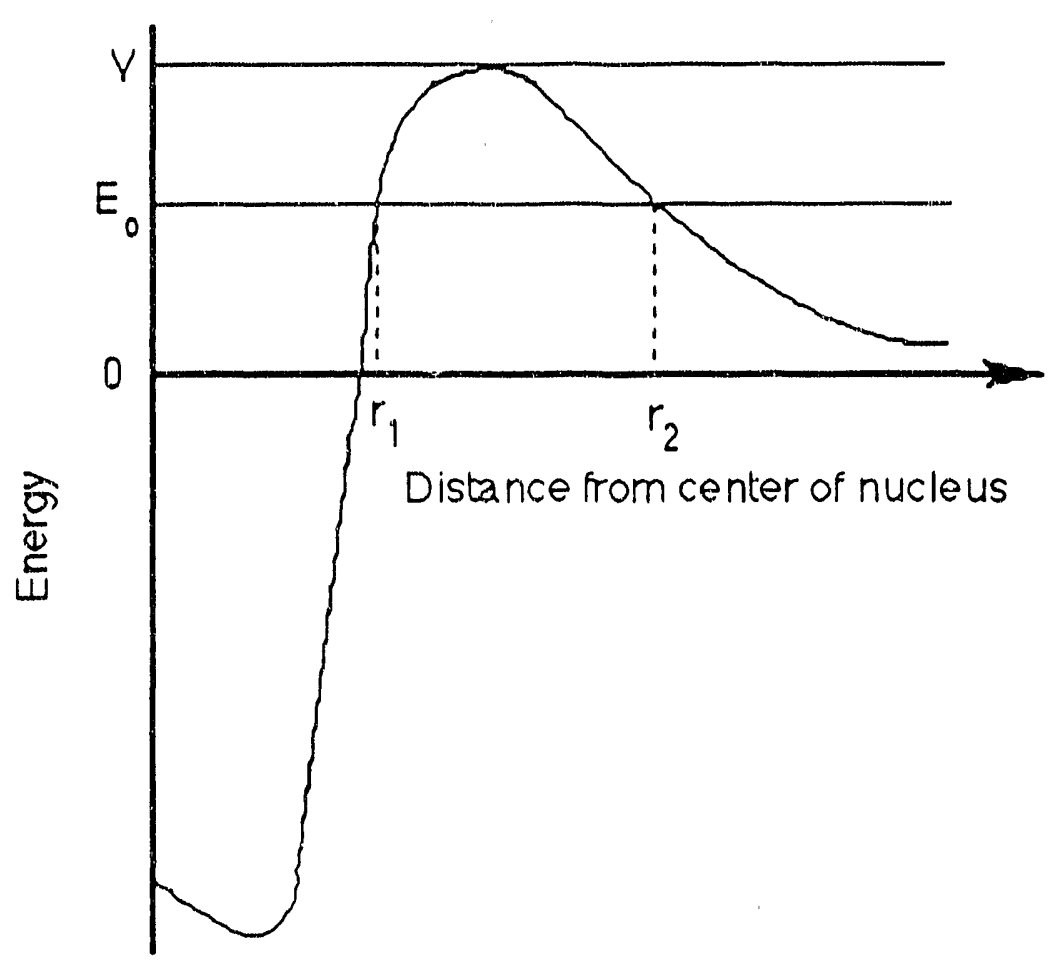

Reference [FRI81]

Unfortunately, ${ }^{259} \mathrm{Fm}$ is the heaviest known fermium isotope. Therefore, the calculation for the HFSF for the 159th neutron is estimated based on a simple ratio. The half-lives for $258 \mathrm{Fm}$ and $259 \mathrm{Fm}$ are $0.37 \mathrm{~ms}$ and 1.5 seconds, respectively. For the 159th neutron, $H F_{S F} \approx 4000$. Multiplying $H F_{S F}$ by the halflife of ${ }^{262} \mathrm{Rf}$ ( $52 \mathrm{~ms}$ ) gives a SF half-life estimate for ${ }^{263} \mathrm{Rf}$ of 260 seconds. In summary, it appears that SF will be the dominant decay mode based on the halflife calculations.

\section{Results and Discussion}

Seven spontaneous fission events were observed in the rutherfordium chemical fraction in 300 experiments. Each experiment had a collection time of 3 
minutes. No alpha events in the 7.0 $-7.2 \mathrm{MeV}$ range were observed with the 7 SF events. Alpha events in this region would be from $252 \mathrm{Fm}, 254 \mathrm{Fm}$, and $255 \mathrm{Fm}$. From yield checks, the fermium isotopes have a SF/a count ratio of $2 / 1$. The maximum fermium alpha event rate from the yield check was 7 events per minute. Therefore, the observed SF events are not contamination from fermium and are assigned to rutherfordium based on the chemical separation. The observed SF events had a half-life of $500 \pm 200$ seconds. The detected alpha events are shown in Figure 67 . No alpha events attributable to ${ }^{263} \mathrm{Rf}$ were detected. This prevented the absolute confirmation of the production of this isotope (see Section 1.B). The production cross section based on the seven SF events is $140 \pm 50 \mathrm{pb}$ somewhat lower than the estimate of $300 \mathrm{pb}$ (see Section 4.B.1).

Alpha events observed at 7.6 $-7.7 \mathrm{MeV}$ are attributed to 214 Po. This isotope is a decay product from the $234 \mathrm{U}$ decay chain and is present in the background. This background interference is near the alpha decay energy of $7.7 \mathrm{MeV}$ predicted for ${ }^{263} \mathrm{Rf}$ by the Möller and Nix masses. This increases the difficulty of observing the alpha decay of $263 \mathrm{Rf}$.

The preliminary results for the search for ${ }^{263} \mathrm{Rf}$ can be used to examine the mass and half-life calculations. No alpha events were observed in the high energy region predicted by the Satpathy masses. The alpha-decay energy of 8.1 $\mathrm{MeV}$ and half-life of 222 seconds predicted using the Satpathy masses would have been readily observable. This seems to discount this mass calculation for use with the heavy elements. The longer half-life predicted by the Möller and Nix masses is consistent with our results. The experimentally determined half-life is very close to the SF half-life estimated from the experimentally determined hindrances [HOF89]. The SF half-life calculated from Lojewski and Baran 
[LOJ88] is off by over an order of magnitude, which is consistent with results for other known odd $A$ rutherfordium isotopes.

\section{Figure 67. Alpha Spectrum From ${ }^{263}$ Rf Search}

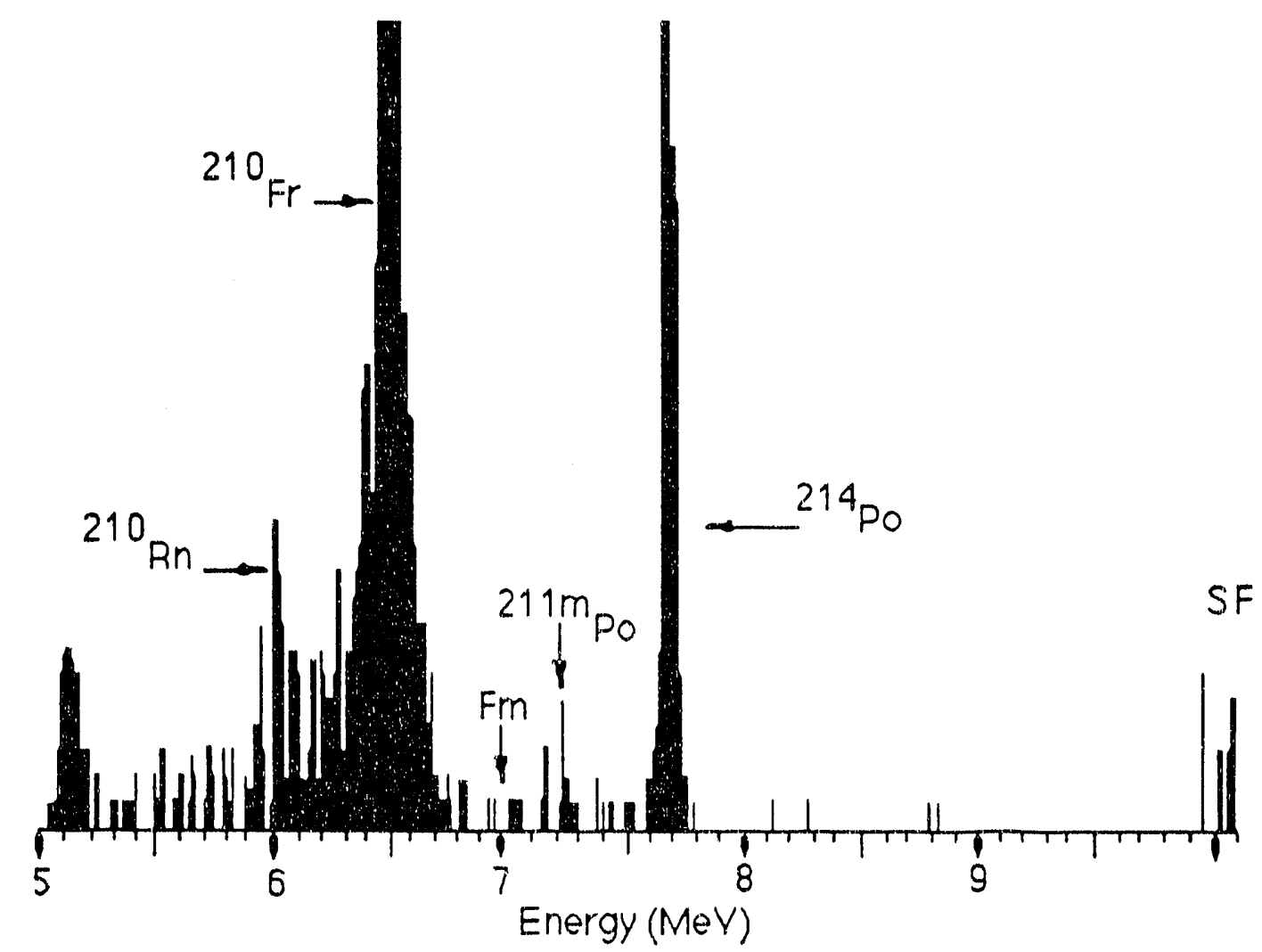

Calculations can be made on the expected number of alpha decays. Assuming the half-life of ${ }^{263} \mathrm{Rf}$ is 500 seconds $\left(\lambda_{\text {Total }}=1.39 \mathrm{E}-3 \mathrm{~s}-1\right)$, the expected number of alpha events as a function of alpha decay energy can be determined using the equation

$$
\lambda_{\text {Tolal }}=\lambda_{\alpha}+\lambda_{S F}
$$

where $\lambda_{\alpha \alpha}$ as a function of decay energy is determined from Figure 65. Equation 28 ignores the contribution from EC decay. The alpha half-life is assumed to be 
greater than 500 seconds. For this reason, the highest alpha decay energy to determine the number of expected alpha decays is $8.0 \mathrm{MeV}\left(\mathrm{t}_{1 / 2}\right.$ hind $=673$ seconds).

The $7 \mathrm{SF}$ events are assumed to be real and have a $\lambda \mathrm{SF}$ calculated from Equation 28. The detector efficiency for alpha decay is half that of SF. This must be accounted for in the number of expected alpha decays by dividing the number of observed SF events by 2 .

$$
\text { \# Expected a Decay }=\frac{\lambda_{\alpha}}{\lambda_{S F}}(7(0.5))
$$

The number of expected alpha (events as a function of energy calculated from Equation 29 is presented in Figure 68. The region of interest for 214Po is excluded from these results.

Using the calculations for the number of expected alpha decays, the probability for observation of zero alpha events as a function of energy can be determined. The Poisson distribution can be used to determine the probability for zero event observation given the expected number shown in Figure 68 [BEV69]. The equation for the probability $(P)$ determination is:

$$
P=\frac{y^{z} e^{-y}}{z !}
$$

where $y$ is the number of expected events and $z$ is the number of observed events. With $z=0$, Equation 30 is simplified to:

$$
P=e^{-y}
$$


The calculations from Equation 31 as a function of energy are presented in Figure 69. The ${ }^{214} \mathrm{Po}$ region is excluded from the figure. These calculations show that the highest probable decay energy is lower than the $214 \mathrm{po}$ region of interest. However, there is an $80 \%-90 \%$ probability for the alpha decay to be in the 214 po region with about half an alpha event expected from the 300 experiments. The probability decreases sharply in the energy range above the 214 po region. At $7.8 \mathrm{MeV}$, the probability of not observing an alpha event is about $50 \%$

This calculation supports the use of the Möller and Nix masses for heavy element calculations. The probability for the ground state to ground state alpha decay energy predicted from the Möller and Nix mass is about $80 \%$. Decay of ${ }^{263} \mathrm{R} f$ to an excited state increases the probability that no alpha events attributable to $263 \mathrm{Rf}$ were observed.

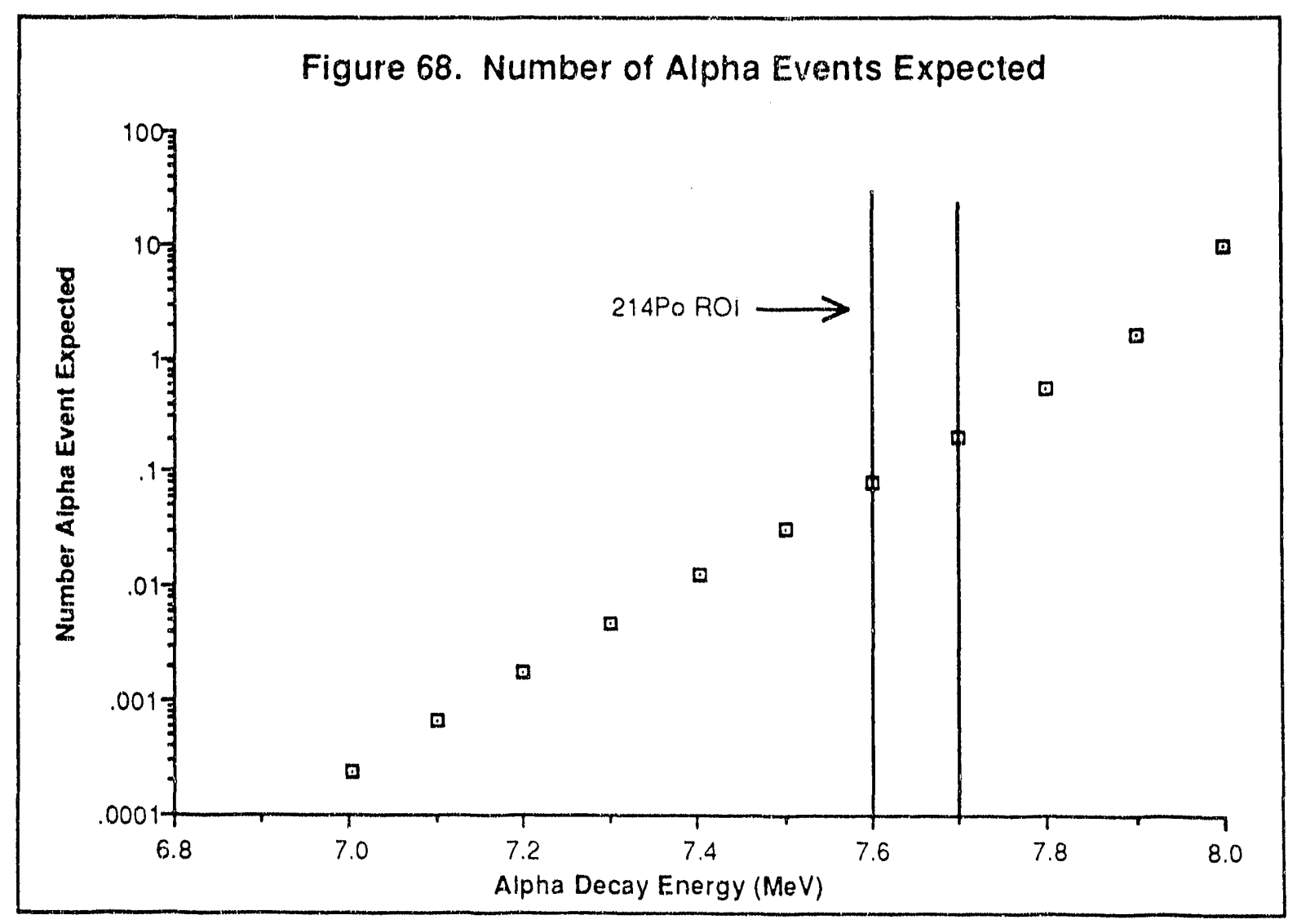




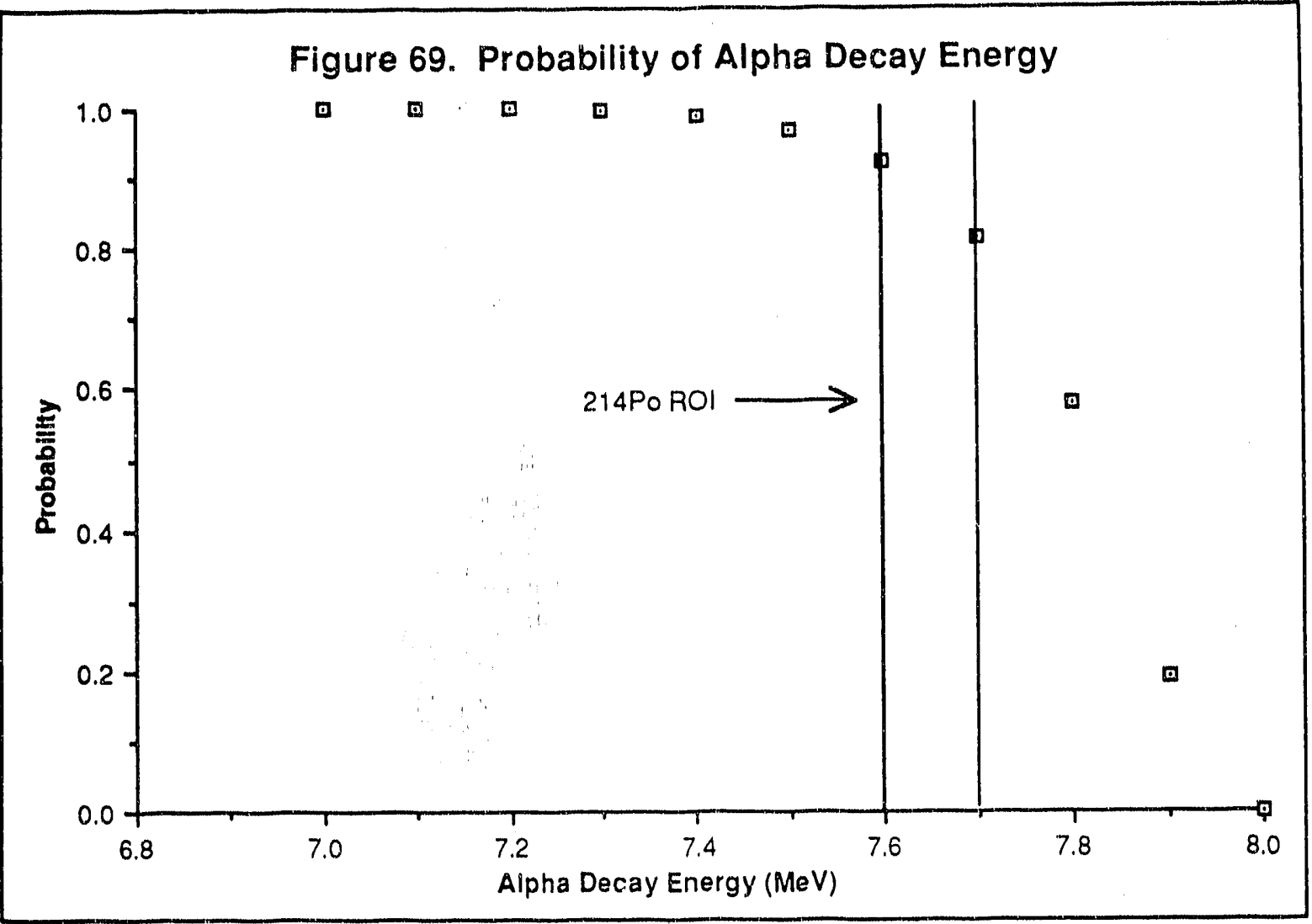

In summery, 7 SF events with a half-life of $500 \pm 200$ seconds and a production cross section of $140 \pm 50 \mathrm{pb}$ were observed in the chemically separated rutherfordium fraction and are tentatively assigned to ${ }^{263} \mathrm{Rf}$. No alpha events attributable to this isotope were observed and might have been masked by contamination from ${ }^{214} \mathrm{Po}$. Since no alpha decay attributable to ${ }^{263} \mathrm{Rf}$ could be observed, positive mass identification of the observed 500-second SF activity cannot be made. 


\section{Chapter 5 . Conclusions}

\section{A. Chemical Studies}

The liquid-liquid extraction studies proved a useful tool in studying rutherfordium. In addition, the TIOA extraction experiments verified previous results on the chemical nature of rutherfordium [HUL80]. The TIOA studies showed that rutherfordium behaves differently than thorium and europium and most similarly to zirconium. This result is further evidence that rutherfordium is a Group 4 element. The major problem with the previous experiment by Hulet et al. was that only 6 rutherfordium events were detected in 44 experiments at 100 $\%$ chemical yield. This was due to the long separation time for the column experiments, about 3 minutes. The TIOA extraction experiments showed that liquid-liquid extraction chemistry can 'se performed very rapidly. The entire chemical separation took about 60 seconds. This increased the event rate to 8 rutherfordium alpha events per hour. The liquid-liquid extraction studies with TIOA are more rapid, and give a higher rate than the previous column experiment by Hulet et al.

Further liquid-liquid extractions of rutherfordium were done with TBP. These extractions showed evidence of a difference between rutherfordium chemistry and the other Group 4 elements for the first time. The results with different chloride concentrations showed that rutherfordium at times behaves more like tetravalent plutonium than zirconium or hafnium. At high chloride concentrations, rutherfordium and plutonium extraction decreased. Under the same conditions, zirconium, hafnium and thorium extraction increased. With an aqueous phase of $12 \mathrm{M} \mathrm{Cl}^{-}: 8 \mathrm{M} \mathrm{H}^{+}$, rutherfordium and plutonium extraction was about $20 \%$, while zirconium, hafnium, and thorium extraction was greater than $80 \%$. In addition, rutherfordium extraction was affected by hydrogen ion concentration, while zirconium and hafnium extraction was not affected by hydrogen ion 
concentration. These results indicate that rutherfordium forms anionic complexes more readily than zirconium or hatrium. In this case, rutherfordium is similar to plutonium. In addition, the extracted complex for rutherfordium differs from that of zirconium or hafnium. This is dramatically demonstrated by the difference in the effect of hydrogen ion concentration on metal extraction. These differences, along with the differences between hahnium and its Group 5 homologs, indicate an unexpected trend in the transactinide elements.

The TTA extraction studies looked at the chemistry of rutherfordium at low $\mathrm{HCl}$ concentrations. This was a departure from the previous liquid-liquid extraction studies, which used a high $\mathrm{HCl}$ concentration. Using the relationship between $K_{d}$ and $K_{\text {eq }}$ for metal extraction, the $\log K_{\text {eq }}$ for rutherfordium was calculated to be $3.18 \pm 0.90$. The results show that the equilibrium constant for rutherfordium is between that of plutonium and thorium. This result was further evidence that differences appear between the transactinide elements and their lighter homologs.

The TTA extraction studies were also used to the estimate the hydrolysis constants of rutherfordium. The hydrolysis constant of rutherfordium was calculated to be greater than the hydrolysis constant of thorium and less than that of zirconium, hafnium, and plutonium. The first four $\log K_{\text {hyd's }}$ for rutherfordium are calculated to be $-2.6 \pm 0.7,-5.9 \pm 1.7,-10.2 \pm 2.9$, and $-14.5 \pm 4.1$. These hydrolysis constants indicates that rutherfordium will not hydrolyze under conditions which zirconium, hafnium, and plutonium will. This result defines an acceptable range for rutherfordium chemistry which differs from zirconium and hafnium chemistry.

Calculation of the ionic radius can be made utilizing the equilibrium constant for rutherfordium derived with TTA extractions. The ionic radius of rutherfordium was calculated to be $91 \pm 4 \mathrm{pm}$ for the 6 -coordinate species and $102 \pm 4 \mathrm{pm}$ for 
the 8-coordinate species. This indicated that the ionic radius was larger than that of zirconium and hafnium. The ionic radius estimates can be compared to values in the literature. The previous estimate for the ionic radius of 6 coordinate rutherfordium from experimental data was $89 \mathrm{pm}$ [GLE89]. The larger ionic radius is also calculated by relativistic effects using MCDF calculations. The relativistic calculations predicted the ionic radius for 6 coordinate rutherfordium to be $80 \mathrm{pm}$ [JOH90]. The increase in the ionic radius for rutherfordium is unusual for the transition metals. Examination of the first 3 rows of the transition metals shows some cases of increasing lonic radius down a column. However, the ionic radius remains quite constant between the second and third rows of the early transition elements. The non-transition elements exhibit an increase in ionic radius going down a column, but this is not an adequate comparison for rutherfordium. The large ionic radius for rutherfordium may point to an increase in ionic radius due to relativistic effects for the fourth row of transition elements. The relativistic effects may change the relative stability of the $7 s, 6 d$ and $7 p$ valence electrons. This, in turn, may alter the expected ionic radius. A recent review of relativistic effects [PYY88] states that the $p_{2 / 3}, d$ and $f$ electrons will be shielded from the high nuclear charge by the contraction of the $s$ and $p_{1 / 2}$ orbitals. The influence of relativistic effects on atomic orbitals should tee contraction and energy stabilization of the $s$ and $p_{1 / 2}$ orbitals and increased radial extension and energy destabilization of the $d$ and forbitals, thus increasing the ionic radius.

\section{B. ${ }^{263} \mathrm{Rf}$ Search}

The search for ${ }^{263} \mathrm{Rf}$ used the chemical separation developed from studying ${ }^{261} \mathrm{Rf}$ with TTA. Seven SF events and no alpha events attributed to ${ }^{263} \mathrm{Rf}$ in the rutherfordium chemical fraction were observed in 300 experiments. The observed SF events had a half-life of $500 \pm 200$ seconds. The cross section of the 
seven events was $140 \pm 50 \mathrm{pb}$. The lack observed an alpha branch attributable to 263Rf prevented the absolute confirmation of the mass of the new isotope, but the chemical separation gives $Z$ confirmation. Alpha events attributed to $214 \mathrm{Po}$, $211 \mathrm{mPo}, 252 \mathrm{Fm}, 255 \mathrm{Fm}, 210 \mathrm{Fr}$ and $210 \mathrm{Rn}$ were observed. The isotope $214 \mathrm{Po}$ is the most troublesome, since it is a natural decay product present in the background and its alpha decay is in a likely energy region for $263 \mathrm{Rf}$ alpha decay.

The preliminary results for the search for $263 \mathrm{Rf}$ can be used to examine the mass and half-life calculations. No alpha events were observed in the energy or half-life region predicted by the Satpathy masses. This seems to discount this mass calculation for use with the heavy elements. The longer half-life predicted by the Möler and Nix masses is supported by these results. The probability calculations also support the use of the Möller and Nix masses for heavy element calculations. The experimentally determined half-life is very close to the SF halflife estimated from the experimentally determined hindrances [HOF89]. The calculated SF half-life from Lojewski and Baran [LOJ88] is too long by over an order of magnitude, which is consistent with the calculations of other known odd A rutherfordium isotopes.

\section{Future Work}

The immediate future work on rutherfordium chemistry should focus on the development of faster and easier chemical separation systems. Gas phase chromatography is being explored as a method of rapid chemical separation. Volatility measurements can be conducted, along with possible measurements of the ionic radius in the gas phase. Chemical bonding of rutherfordium to surfaces is also being investigated. This method can be used to further study the hydrolysis and other basic chemical properties of rutherfordium as well as for rapid separation in searches for new rutherfordium isotopes. Rapid and automated ion-exchange methods can be developed to further study the aqueous 
chemistry of rutherfordium. The ionic radius can be remeasured using ionexchange techniques.

The long term future of rutherfordium and other transactinide element research resides with the development of methods for production of new and longer lived isotopes. One method is the development of radioactive nuclear beams [CAS91]. The use of neutron rich beams may allow the production of heavier, and hence more stable, transactinide elements. In the case of rutherfordium, beams of 19.220 can be produced and reacted with a $248 \mathrm{Cm}$ target. Isotopes up to ${ }^{267} \mathrm{Rf}$ can be produced by this method. Unfortunately, beam intensities are predicted to be rather low. Methods for increasing the beam flux will need to be develuped to make the this method productive.

The use of heavier targets will also aid in the production of heavier transactinide elements. Californium and einsteinium targets can be produced. These elements have a higher $Z$ than curium, facilitating the production of transactinide elements. In addition, $250 \mathrm{Cm}$ may be isolated from nuclear test debris. The use of this target will make the production of heavier isotopes of rutherfordium and other transactinide elements possible. Since these isotopes will be longer lived, more chemical studies can be conducted, thereby opening a new field for transactinium element research. 


\section{References}

[ALO74] Alonso, J., Gmelin Handbuch der Anorganishen Chemie. Ib, 28 (1974).

[AUM74] Aumann, D.C., Müllen, G., Nucl. Instr. Meth. 75, 115 (1974).

[BAE76] Baes, C.F., and Mesmer, R.E., The Hydrolysis of Cations; Wiley Interscience, New York, 152-191 (1976).

[BEC70] Beck, M.T., Chemistry of Complex Equilibria; Van Nostrand, London, 45 (1970).

[BAG76] Bagawde, S.V., Ramakrishna, V.V., and Patil, S.K., J. Inorg. Nucl. Chem. 38, 1339 (1976).

[BEL.75] Belousov, E.A., and Zakharova, L.Yu., Russ. J. Phys. Chem. 49 , 1965 (1975).

[BEV69] Bevington, P.R., Data Reduction and Error Analysis for the Physical Sciences; McGraw Hill, New York, 36-43 (1969).

[BLU45] Blumenthal, E., and Herbert, J.B., Trans. Faraday Soc. 41,611 (1945).

[BOR20] Born, M., Z. Physik. 1, 45 (1920).

[BRE84] Brewer, L., High Temp. Sci. 17, 1 (1984).

[BRU89] Bruchertseifer H., JINR Rap. Comm., 34, 89 (1989).

[CAS91] Casten, R.F. ed., The Isospin Laboratery: Research Oppertunities with Radioactive Nuclear Beams, LALP 91-51 (1991).

[CLE70] Cleveland, J.M., The Chemistry of Plutonium; Gordon and Breach Science Publishers, New York, 176 (1970).

[COH75] Cohen, N., Guilmette, R., Bioinorganic Chem. 5, 203 (1975).

[COL78] Coleman, C.F., Leuze, R.E., J. Tenn. Acad. Sci. 53, 102 (1978). 
[CON49] Connick, R.E., and McVey, W.H., J. Amer. Chem. Soc. Z1, 3182 (1949).

[CON29] Condon, E.U., and Gurney, R.W., Phys. Rev. 33, 127 (1929).

[CO052] Cook, E.H., and Taft, R.W., J. Amer. Chem. Soc., 74, 6103 (1952).

[COT72] Cotton, F.A., and Wilkinson, G., Advanced Inerganic Chemistry;

Wiley and Sons, New York 820-830 (1972).

[DES80] Desclaux, J.P., and Fricke, B., J. Phys. (Paris) 41943 (1980).

[DUR75] Durbin, P., Health Physics 29, 495 (1975).

[ERT73] Erten,H.N., J. Radioanal. Chem. 14, 343 (1973).

[EVA72] Evans, J.E., Lougheed, R.W., Coops, M.S., Hoff, R.W., Hulet, E.K., Nucl. Instr. Meth. 102, 389 (1972).

[FLE64] Flerov, G.N., Oganessian, Yu., Ts., Lobanov, Yu. V., Kuznetsov, V.I., Drui, V.A., Perelygin, V.P., Gavrilov, K.A., Tretyakova, S.P. and Plotko, V.M., Phys. Lett. 13, 73 (1964).

[FRI81] Friedlander, G., Kennedy, J.W., Macias, E.S., and Miller, J.M., Nuclear and Radiochemistry; 3rd ed., Wiley and Sons, New York (1981). [GAM29] Gamov, G, Z. Phys. 52, 510 (1929).

[GEl11] Geiger, H., and Nuttal, J.M., Phil. Mag., 22, 613 (1911).

[GHI69] Ghiorso, A., Nurmia, M., Harris, J., Eskola, K., and Eskola, P., Phys. Rev. Lett. 22, 1317 (1969).

[GHI70] Ghiorso, A., Nurmia, M., Eskola, K., and Eskola, P., Phys. Lett. $32 \mathrm{~B}$. $95(1970)$.

[GLE89] Glebov, V.A., Kasztura, L., Nefedov, V.S., and Zhuikov, B.L., Radiochim Acta 46, 113 (1989).

[GRE84] Greenwood, N.N. and Earnshaw, A., Chemistry of the Elements;

Pergamon Press, New York, $1444-5$ (1984). 
[GRE91] Gregorich, K.E., Türler, A., Czerwinski, K.C., Kadkhodayan, B.A., Hannink, N.J., Lee, D.M., Nurmia, M., Kreek, S.A., Kacher, C.D., Hoffman, D.C, Zimmermann, M.N., Kratz, J.V., Gober, M.K., Schädel, M., Brüchle, W., Schimpf, E., Gäggeler, H., Jost, D., Kovacs, J., Scherer, U.W., and Weber, A., Extraction of Element 105 inte Diisobutylcarbinol Lawrence Berkeley Laboratory Nuclear Science Division Annual Report, 72 (1991). [GRO76] Groote, H., Hilf, E.R., and Takahashi, Atom. Data Nucl. Data Tables 17. $418(1976)$.

[HAR81] Harris, W.R., Raymond, K.N., and Weitl, F.L., J. Am. Chem. Soc. 103, 2667 (1981).

[HAR79] Harris, W.R., and Raymond, K.N., J. Am. Chem. Soc. 101, 6534 (1979).

[HAT90] Hatsukawa, Y., Ph.D. Thesis, Decay Properties of Einsteinium Isotopes and Alpha Decay Systematics Tokyo Metropolitan University (1990).

[HAT90A] Hatsukawa, Y., Nakuhara, H., and Hoffman, D.C., Phys. Rev. C. 42 , 674 (1990).

[HAU88] Haustein, P.E. ed., Atom. Data Nucl. Data Tables 39, 289 (1988).

[HER45] Hertzberg, G., Infrared and Raman Spectra; Van Nostrand, New York, 45 (1945).[HUB90] Hubert, F., Bimbot, R. and Gauvin, H., Nucl.

Data Tables. 46, 1 (1990).

[HOF89] Hoffman, D.C., Spontaneous Fission Properties and Lifetime

Systematics, LBL.-26975, (1989).

[HUF49] Huffman, E.H., and Beaufait, L.J., J. Amer. Chem. Soc., Z1, 3179 (1949).

[HUL80] Hulet, E.K., Lougheed, R. W., Wild, J. F., Landrum, J. H., Nitschke, J. M., Ghiorso, A., J. Inorg. Nucl. Chem 42,79 (1980). 
[HUH72] Huheey, J.E., Inorganic Chemistry: Principles of Structure and Beactivity; Harper and Rowe, New York, 418 (1972).

[HYD87] Hyde, E.K., Hoffman, D.C., and Keller Jr., O.L., Radiochim Acta 42(2), 57 (1987).

[JOH90] Johnson, E., Fricke, B. Keller, Jr. O.L., Nestor, Jr. C.W., and Tucker, T.C., J. Chem. Phys. 23, (1990).

[KAT86] Katz, J.J., Seaborg, G.T., and Morss, L.R., The Chemistry of the Actinide Elements: Volume?; Chapman and Hall, New York, 1512-1519 (1986).

[KAT86A] Katz, J.J., Seaborg, G.T., and Morss, L.R., The Chemistry of the Actinide Elements: Volume 1; Chapman and Hall, New York, 525-534 (1986).

[KEL84] Keller, Jr., O.L., Radiochim. Acta 37, 169 (1984).

[KER60] Kertes, A.S., J. Inorg. Nucl. Chem. 14, 104 (1960).

[KIN51] King, E.L., and Reas,W.H., J. Amer. Chem. Soc. 73, 1806 (1951).

[KPA92] Kratz, J.V., Gober, M.K., Zimmerman, H.P., Schädel, M., Brüchle, W., Schimpf, E., Gregorich, K.E., Türler, A., Hannink, N., Czerwinski, K.R., Kadkhodayan, B., Lee, D.M., Nurmia, M.J., Hoffman, D.C., Gäggeler, H., Jost, D., Scherer, U.W., and Weber, A., Phys. Rev. C. 45 (1992) in press.

[LIR76] Liran, S., and Zeldes, N., Atom. Data Nucl. Data Tables 17, 431 (1976).

[LED78] Lederer, C.M., and Shirley, V.S., eds., Table of the Isotopes, 7thed, John Wiley and Sons, Inc., New York, 1406 (1978).

[LER87] Leres, R.G., RAGS-an "Oif-the-Shelf" Hardware and System Software Small Data Acquisition Computer, LBL-24808 (1987).

[LOJ88] Lojewski, Z., and Baran, A., Z. Phys. A., Atornic Nuclei. 329, 161 (1988). 
[LOU86] Lougheed, R.W., Lawrence Livermore National Laboratory Nuclear Chemistry Division Annual Report (UCAR10062/86), 4 (1986).

[LOU87] Lougheed, R.W., Lawrence Livermore National Laboratory Nuclear Chemistry Division Annual Report (UCAR10062/87), 4 (1987).

[MAC73] Macdermott, T.E., Coord. Chem. Rev. 11, 1 (1973).

[MAE61] Maeck, W.J., Booman, G.L., Kussy, M.E., and Rein, J.E., Anal. Chem. 33, 1775 (1961).

[MAE62] Maeck, W.J., Booman, G.L., Kussy, M.E., and Rein, J.E., Anal. Chem. 34, 212 (1962).

[MAR69] Marcus, Y., and Kertes, A.S., len Exchange and Solvent Extraction of Metal Complexes; Wiley-Interscience, New York, 741 (1969).

[MAR69A] Marcus, Y., and Kertes, A.S., Len Exchange and Solvent Extraction of Metal Complexes; Wiley-Interscience, New York, 668-685 (1969).

[MAR69B] Marcus, Y., and Kertes, A.S., Len Exchange and Solvent Extraction of Metal Complexes; Wiley-Interscience, New York, 62 (1969).

[MAR69C] Marcus, Y., and Kertes, A.S., Len Exchange and Solvent Extraction ef Metal Complexes; Wiley-Interscience, New York, 500-510 (1969). [MAR52] Martell, A.E., and Calvin, M., Chemistry of the Metal Chelate Cemoounds; Prentice-Hall, New York, 191-202 (1952). [MÖL88] Möller, P., Myers, W.D., Swiatecki, W.J., and Treiner, J., At. Data. Nucl. Data Tables. 39, 225 (1988). [MÖL88A] Möller, P, and Nix, J.R., At. Data Nucl. Data Tables. 39, 213 (1988). [MÖL81] Möller, P., and Nix, J.R, At.Data Nucl. Data Tables 26, 165(1981). [MUL75] Müllen, Aumann, D.C., Nucl. Instr. Meth. 128, 425 (1975). [MYE76] Myers, W.D., Atom. Data Nucl. Data Tables 17, 411 (1976). [NAl62] Naito, K., and Suzuki, T., J. Phys. Chem. 66, 983 (1962). 
[NOR70] Northcliff, L.C., and Schilling, R.F., Nucl. Data Tables. Z(3-4), 235 (1970).

[PAN86] Panda, C.R., Solvent Extraction and Ion Exchange 4(5), 969 (1986).

[PAT80] Patil, S.K., Ramakrishna, V.V., Kartha, P.K.S., and Gudi, N.M., J.

Radioanal. Chem., 59, 331 (1980).

[PEP56] Peppard, D.F., Mason, G.W., and Maier, J.L., J. Inorg. Nucl. Chem. 3, 215 (1956).

[POS61] Poskanzer, A.M., and Foreman, Jr., B.M., J. Inorg. Nucl. Chem. 16 , 323 (1961).

[PYY88] Pyykkö, P., Chem. Rev. 88, 563 (1988).

[RAY84] Raymond, K.N., Freeman, G.E., and Kappel, M.J., Inorg. Chim. Acta 24, 193 (1984).

[RAM78] Ramanujam, A., Nadkarni, M.N, Ramakrishna, V.V., and Patil, S.K., J. Radioanal. Chem. 42, 349 (1978).

[REI50] Reid, J.C., and Calvin, M., J. Amer. Chem. Soc. 72, 2948 (1950).

[REU83] Reus, U., and Westmeier, W., Nucl. Data Tables. 29, 193 (1983).

[SAl63] Saito, N., and Yamasaki, A., Bull. Chem. Soc. Jap. 36, 1055 (1963).

[SAM80] Sambasiva-Reddy, A., and Krishna Reddy, L., Separation Science and Technology 15(5), 1263 (1980).

[SAT88] Satpathy, L., and Nayak, R.C., Atom. Data Nucl. Data Tables 32, 241 (1988).

[SCH84] Schultz, W.W, Navratil, J.D., and Talbot, A.E., Eds., Science and Iechnology of Tributy! Phosphate Voumne 1; CRC Press, Boca Raton, FL, 164-166 (1984).

[SCH84A] Schultz, W.W, Navratii, J.D., and Talbot, A.E., Eds., Science and Iechnology of Tributyl Phosphate Volume 1; CRC Press, Boca Raton, FL, 139-146 (1984). 
[SCH84B] Schultz, W.W, Navratil, J.D., and Talbot, A.E., Eds., Science and Iechnology of Tributyl Phosphate Volume 1; CRC Press, Boca Raton, FL, 120-122 (1984).

[SEA90] Seaborg, G.T., and Loveland, W.D., The Elements Bevond Uranium; Wiley Interscience, New York, 51 (1990).

[SEE76] Seeger, P.A., and Howard, W.M., Atom. Data Nucl. Daia Tables 17. 428 (1976).

[SEK67] Sekine, T., and Dyrssen, D., J. Inorg. Nucl. Chem. 29, 1457 (1967).

[SHA76] Shannon, R.D., Acta Crystal. A32(5), 751 (1976).

[SIL70] Silva, R., Harris, J., Nurmia, M., Eskola, K., Ghiorso, A., Inorg. Nucl. Chem. Lett. 6, 871 (1970).

[SMI72] Smith, V.H., Health Physics 22, 765 (1972).

[SOL73] Solovkin, A.S., and Tsvetkova, S.V., Russ. Chem. Rev. 31, 655 (1973).

[SPA88] Spanier, L., and Johansson, A.E., Atom. Data Nucl. Data Tables 39, 259 (1988).

[STA64] Stary, J., The Solvent Extraction of Metal Chelates; Macmillan Company, New York, 71-77 (1964).

[TAA61] Taagepera, R., and Nurmia, M., Ann. Acad. Sci. Fenn. Ser. A. 78, 1 (1961).

[TAC88] Tachibana, T., Uno, M., Yamada, M., and Yamada, S., Atom. Data Nucl. Data Tables 39, 251 (1988).

[TAU60] Taube, M., J. Inorg. Nucl. Chem. 12, 134 (1960).

[TAY72] Taylor, D.M., Health Physics 22, 575 (1972).

[TRE64] Tremillon, B., Bull. Chem. Soc. France 1057 (1964).

[VD061] Vdovenko, V.M., Lipovskii, A.A., Kuzina, M.G., Radiokhimiya, 3, 403 (1961). 
[VIO66] Viola, V.E., and Seaborg, G.T., J. Inorg. Nucl. Chem. 28, 741 (1966).

[WAL89] Walker, F.W., Parrington, J.R., and Feiner, F. eds., Chart of the

Nuclides; General Electric, San Jose, CA, 13 (1984).

[WAP59] Wapstra, A.H., Nijgh, G.J., and Van Lieshout, R., Nuclear

Spectroscopy Tables: North-Holland, Amsterdam, 37 (1959).

[WE179] Weitl, F.L., Lab notebook I, 88 (1979).

[WEl80] Weitl, F.L.; Raymond, K.N., J. Am. Chem. Soc. 102, 2289 (1980).

[WEl81] Weitl, F.L.; Raymond, K.N.; Durbin, P.W., J. Med. Chem. 24, 203 (1981).

[WIL62] Wilson, A.M., Churchill, L., Kikuk, K., and Hovsepian, P. , Anal.

Chem. 34, 203 (1962).

[YOU53] Young, R.S., Industrial Inorganic Analysis; Chapman and Hall, London, 58 (1953).

[ZVA67] Zvara, I., Chuburkov, Yu. T., Caletka, R., Zvarova, T. S.,

Shalayevsky, M. R., Shilov, B. V., J. Nucl. Energy 21, 601 (1967).

[ZVA70] Zvara, I., Chuburkov, Yu. T., Belov, V. Z., Bulkanov, G. V.,

Zukhvataev, B. B., Zvarova, T. S., Maslov, O. D., Caletka, R., Shalayevsky,

M. R., J. Inorg. Nucl. Chem 32, 1885 (1970).

[ZVY65] Zvyagintsev, O.E., Frolov, Yu. G., Chin-pang, Ch., and Valkov, A.V., Zh. Neorg. Khim. 10, 981 (1965). 

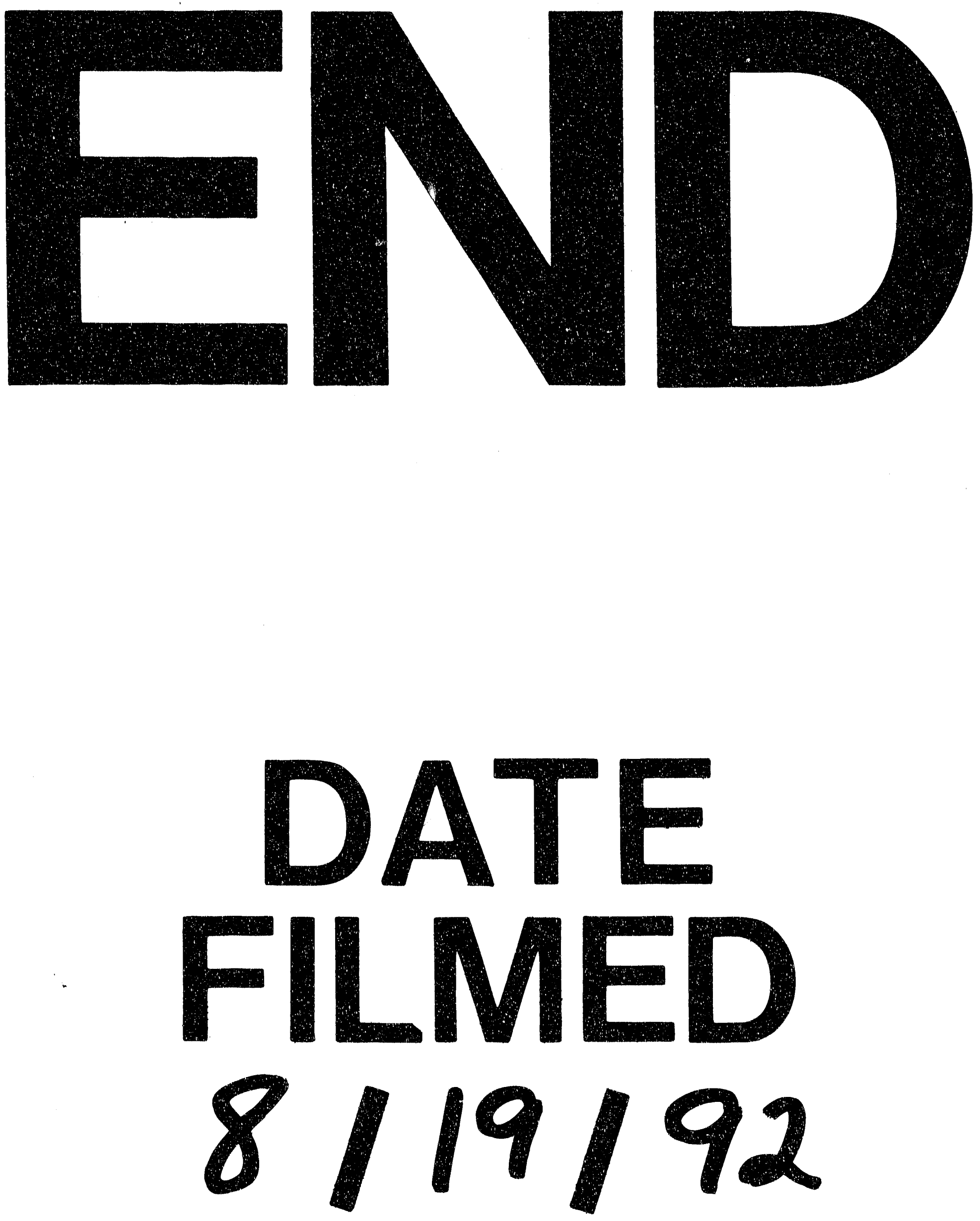
